\title{
Droplet Impact Sub-cavity Histories and PDPA Spray Experiments for Spray Cooling Modeling
}

Nicholas Lee Hillen

West Virginia University

Follow this and additional works at: https://researchrepository.wvu.edu/etd

\section{Recommended Citation}

Hillen, Nicholas Lee, "Droplet Impact Sub-cavity Histories and PDPA Spray Experiments for Spray Cooling Modeling" (2013). Graduate Theses, Dissertations, and Problem Reports. 3387.

https://researchrepository.wvu.edu/etd/3387

This Thesis is protected by copyright and/or related rights. It has been brought to you by the The Research Repository @ WVU with permission from the rights-holder(s). You are free to use this Thesis in any way that is permitted by the copyright and related rights legislation that applies to your use. For other uses you must obtain permission from the rights-holder(s) directly, unless additional rights are indicated by a Creative Commons license in the record and/ or on the work itself. This Thesis has been accepted for inclusion in WVU Graduate Theses, Dissertations, and Problem Reports collection by an authorized administrator of The Research Repository @ WVU. For more information, please contact researchrepository@mail.wvu.edu. 


\title{
DROPLET IMPACT SUB-CAVITY HISTORIES \\ AND PDPA SPRAY EXPERIMENTS FOR SPRAY COOLING MODELING
}

By

\author{
NiCHOLAS LEE HiLLEN
}

Thesis submitted to the

Benjamin M. Statler College of Engineering and Mineral Resources at West Virginia University in partial fulfillment of the requirements for the degree of

\author{
Master of Science \\ in \\ Mechanical Engineering \\ Committee: \\ John M. Kuhlman, Ph.D., Chair \\ Donald D. Gray, Ph.D. \\ Gary J. Morris, Ph.D.
}

Department of Mechanical and Aerospace Engineering West Virginia University

2013

Keywords: Spray Cooling; Single Drop; PDPA; Spray; Splashing;

Optical Measurements; High-speed Video

Copyright 2013 Nicholas L. Hillen 


\section{AbSTRACT \\ Droplet Impact Sub-cavity Histories and PDPA Spray Experiments for Spray Cooling Modeling NiCHOLAS L. HILLEN}

Spray cooling is a topic of current interest for its ability to uniformly remove high levels of waste heat from densely packed microelectronics. It has demonstrated the ability to achieve very high heat fluxes, up to $500 \mathrm{~W} / \mathrm{cm}^{2}$ with water as the coolant, making it an attractive active thermal management tool.

Full Computational Fluid Dynamic (CFD) simulations of spray cooling are infeasible due to the complexity of the spray (drops fluxes of $10^{6} \mathrm{drops} / \mathrm{cm}^{2}-\mathrm{sec}$ ) and heater surface physics requiring impractical resources. Thus a Monte-Carlo (MC) spray cooling simulation model based on empirical data is under development to serve as a cost effective design tool. The initial MC model shows promise, but it lacks additional physics necessary to predict accurate heat fluxes based on nozzle conditions and heated surface geometry.

This work reports spray and single drop experiments with the goal of computing the volume beneath a droplet impact cavity (the sub-cavity volume) created by a single impinging droplet on an initial liquid layer. A Phase Doppler Particle Analyzer (PDPA) was utilized to characterize a spray of interest in terms of integrated global Weber, Reynolds, and Froude numbers for varying flow conditions. Results showed that the spray droplet diameters decreased and velocities increased with increasing nozzle gage pressure. A relevant test plan for the single drop experiments has been created from the measured PDPA spray profiles combined with residual spray film thickness measurements from literature resulting in: $140 \leq$ $W e \leq 1,000,1,200 \leq R e \leq 3,300$, and $0.2 \leq h_{0}{ }^{*} \leq 1.0$. Froude numbers were not able to be matched for the current single drop experiments (spray: $32,800 \leq F r \leq 275,000$ ).

Liquid film thicknesses under the cavity formed by a single droplet have been measured versus radius and time via a non-contact optical thickness sensor for the selected range of dimensionless numbers ( $W e, R e$, and $h_{0}{ }^{*}$ ). Sub-cavity radius histories have also been analyzed utilizing high-speed imagery techniques to create the cavity thickness traverse profiles. Time dependent sub-cavity volumes have been computed by integrating these subcavity liquid film thicknesses versus radius at various times. It is found that higher $W e$ and lower $h_{0}{ }^{*}$ result in a more radially uniform sub-cavity surface contour versus time, except for thinner liquid film regions which are observed near the outer bottom cavity radius. The subcavity volume was found to be nearly constant for a majority of the cavity lifetime and increased with $W e$ and $h_{0}{ }^{*}$. These results will be incorporated into the MC model to improve its predictive capability in future work.

In addition, splashed droplet diameters and velocities have been extracted from PDPA data for a spray impinging normal to a smooth surface. It was found that the splashed droplets had sizes which were similar to the impinging spray droplets, and had velocities that never exceeded $3 \mathrm{~m} / \mathrm{s}$. The splashed droplet results have a negligible contribution to cavity formations due to their low Weber number. This splashing data has been detailed for future implementation into the MC model in terms of mass conservation in the liquid film. 


\title{
DROPLET IMPACT SUB-CAVITY HISTORIES AND PDPA SPRAY EXPERIMENTS FOR SPRAY COOLING MODELING
}

\author{
Nicholas Lee Hillen \\ Thesis submitted to the \\ Benjamin M. Statler College of Engineering and Mineral Resources \\ at West Virginia University \\ In partial fulfillment of the requirements for the degree of \\ Master of Science \\ in \\ Mechanical Engineering \\ Department of Mechanical and Aerospace Engineering
}

APPROVAL OF THE EXAMINING COMMITTEE

Donald D. Gray, Ph.D.

Gary J. Morris, Ph.D.

Date

John M. Kuhlman, Ph.D., Chair 


\section{FOR \\ HALEY \\ AND \\ MY FAMILY}

Page iv 


\section{CONTENTS}

Abstract___ ii

Contents __ v

Figures__viii

Tables__xiii

Nomenclature __ xiv

CHAPTER 1: Introduction__ 1

CHAPTER 2: Literature Review __ 10

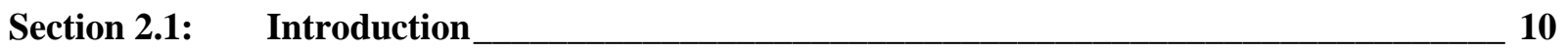

Section 2.2: Current Monte-Carlo Spray Cooling Model ___ 11

Section 2.3: Single Drop Impingement ___ 15

Section 2.3.1: Crown Morphology and Formation ___ 16

Section 2.3.2: Splashing ___ 18

Section 2.3.3: Splashed Droplet Characteristics ___ 20

Section 2.3.4: Spray Impingement Versus Single Droplet Impingement __ 21

Section 2.4: Cavity Dynamics________________ 22

Section 2.4.1: Sub-cavity Film Thickness ___ 23

Section 2.5: Composition of Drop and Initial Layer Fluid___ 25

Section 2.6: Residual Spray Film Thickness ___ 26

Section 2.7: PDPA Background _____ 26

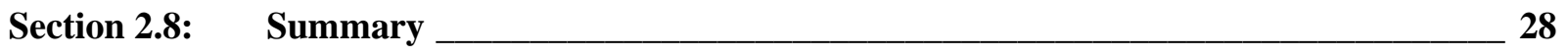

CHAPTER 3: $\quad$ Spray Experiments Apparatus and Procedure __ 29

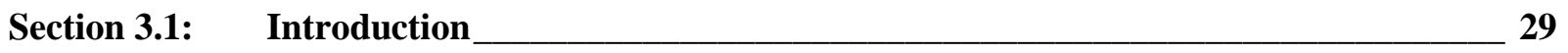

Section 3.2: Spray Apparatus_______________ 29

Section 3.2.1: Spray Impact Surface for Splash Droplet Experiments ___ 30

Section 3.3: PDPA Setup_____ 31

Section 3.3.1: Droplet Size Validation ___ 33

Section 3.4: $\quad$ Spray PDPA Characterization Procedure: Without the Spray Impact Surface 34

Section 3.5: Splashed Droplets Methodology ___ 36

Section 3.5.1: Vertical Edge Splash Droplet Methodology ___ 39

CHAPTER 4: $\quad$ Single Drop Experiments Apparatus and Procedures ___ 43

Section 4.1: Introduction______ 43

Section 4.2: Single Drop Sub-cavity Experiments Apparatus ___ 44

Section 4.3: Initial Preliminary Investigation _______ 46

Section 4.4: Single Drop Sub-cavity Radius Video Methodology ___ 49 
Section 4.4.1: Sub-cavity Radius Image Processing ___ 51

Section 4.5: Single Drop Sub-cavity Methodology ___ 52

Section 4.5.1: Single Droplet Image Processing ___ 56

CHAPTER 5: $\quad$ Single Drop Sub-Cavity Volume Calculation Methodology__ 57

Section 5.1: Introduction___ 57

Section 5.2: Sub-cavity Integration Method ___ 57

Section 5.3: Sub-cavity Volume Noise Removal ____ 58

Section 5.4: Sub-cavity Volume Radial Film Thickness Profile Preprocessing _ _ 59

Section 5.5: Sub-cavity Volume Iterative Technique ___ 60

Section 5.6: Sub-cavity Volume Accuracy Check__ 63

CHAPTER 6: SPray Results and Discussion _ _ 65

Section 6.1: Introduction___ 65

Section 6.2: Spray Results and Discussion: Without Spray Impact Surface Installed ___ 66

Section 6.2.1: Spray Trends and Effects of Varying the Nozzle Flow Condition ___ 68

Section 6.2.2: Uncertainty Analysis of PDPA Spray Data (No Target Surface) ___ 71

Section 6.2.3: Effects of Varying the Standoff Distance ___ 78

Section 6.2.4: Spray Nozzle Droplet Size and Velocity Characterization ___ 79

Section 6.3: Splash Results and Discussion: Spray Impact Surface Installed ___ 83

Section 6.3.1: Comparison of Spray with and without the Impact Surface Installed____ 83

Section 6.3.2: Separated Splashed Droplet Components: Impact surface Installed_____ 85

Section 6.3.3: Effects of Varying the Nozzle Flow Conditions on the Splashed Droplet Components___ 88

Section 6.3.4: Comparison of $x$-axis Splash Results with Vertical Edge Splash Results ___ 90

Section 6.3.5: Splashed Droplets Characterized in Dimensionless Numbers ___ 91

Section 6.4: Volume Flux_____ 93

Section 6.5: Development of Test Matrix for Single Drop Sub-cavity Experiments ___ 94

CHAPTER 7: $\quad$ Single Drop Results and Discussion __ 97

Section 7.1: Introduction____ 97

Section 7.2: Preliminary Investigation Results and Discussion ___ 98

Section 7.2.1: Experimental and 2D Simulation Comparison ___ 103

Section 7.3: Single drop Sub-cavity Initial Droplet Parameters __ 105

Section 7.4: Sub-cavity Radius Results and Discussion ___ 106

Section 7.5: Single Drop Cavity Flow Visualization ____ 109

Section 7.5.1: Additional Splashing Observations____ 113

Section 7.6: Single Drop Centerline Sub-cavity Film Thickness _____ 115

Section 7.7: $\quad$ Single Drop Cavity Film Thickness Surface Contour Maps___ 118

Section 7.8: Sub-cavity Volume Results and Discussion ___ 123

CHAPTER 8: Conclusions and Future Work__ 128 
Section 8.1: Conclusions ___ 128

Section 8.2: Future Work ___ 132

Acknowledgements___ 134

REFERENCES _ 135

Appendix A: CAD Drawings ___ 139

Appendix B: Additional PDPA Spray Data (No Impact Surface) ___ 144

Appendix C: Additional PDPA Splashing Data (With Impact Surface) _ 150

Appendix D: Glycerol-Water Solution Methodology and CHR Thickness Validation__ 168

Appendix E: Additional Single Droplet Sub-cavity Results___ 173 


\section{FiguRES}

Figure 1.1: Full cone water spray generated by a Spraying Systems FullJet 1/8-G nozzle on an unheated $5.08 \mathrm{~cm}$ diameter surface (Hillen et al. 2013).

Figure 1.2: Single drop impingement into an unheated initial liquid surface and its components (Hillen et al. 2013 ). 6

Figure 2.1: Simulated heater surface from the MC Spray Cooling model (Kreitzer, 2010). ___ 14

Figure 2.2: Droplet impact angle. ____ 15

Figure 2.3: Incident beams crossing creating the measuring volume._______ 27

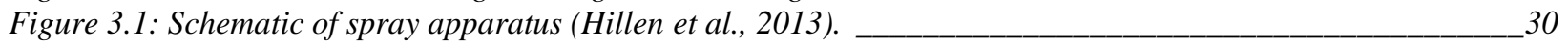

Figure 3.2: Spray testing $5.08 \mathrm{~cm}$ diameter impact surface apparatus with nozzle. _____ 31

Figure 3.3: PDPA transmitting and receiving optics and traverse system. a) Optics mounted on the tri-axis traverse. $b$ ) Mounted optics configured in back-scatter mode (Menchini, 2013)._____ 32

Figure 3.4: Diameter data validation graphs. a) Intensity validation curve. b) Diameter difference validation (Hillen et al. 2013). __ 34

Figure 3.5: Coordinate system for spray axis and droplet coordinate system (Hillen et al., 2013). ___ 35

Figure 3.6: Sketch explaining the z positioning of the measuring volume to avoid interference from the impact surface and fluid ring buildup. ___ 37

Figure 3.7: Comparison of raw PDPA spray data of droplet diameter plotted vs. axial velocity. a) $R_{s}=0, z=3.2 \mathrm{~cm}$ for the 2.76 bar spray without the impact surface, $b) R_{s}=0, z=3.2 \mathrm{~cm}$ for the 2.76 bar spray with the impact surface.

38

Figure 3.8: Separation of the spray and splash regions into the droplet sub-categories. ___ 39

Figure 3.9: Spray and splash droplet diameters versus axial velocity at $z=3.6 \mathrm{~cm}$ at the three nozzle flow conditions. $a$ ) 1.38 bar and $29.5 \mathrm{~L} / \mathrm{hr}$. b) $2.76 \mathrm{bar}$ and $43.5 \mathrm{~L} / \mathrm{hr}$. c) $4.14 \mathrm{bar}$ and $54.5 \mathrm{~L} / \mathrm{hr}$.

40

Figure 3.10: Histograms of the separated droplet diameter data for a spray at 4.14 bar impinging on impact surface at $R_{s}=0 \mathrm{~cm}$ and $z=3.65 \mathrm{~cm}$. a) Spray droplets. b) Ejected splashed droplets. c) Returning splashed droplets. d) Absolute splashed droplets.

41

Figure 3.11: Spray apparatus impinging onto the impact surface with the laser probe volume traversing along the z-axis to the side. The splashed secondary droplets ejected outside of the spray cone that are being measured are circled in red.

Figure 4.1: Schematics of the single drop experimental apparatus. a) Entire apparatus. b) Cross section of the impact stand and optical probe (Hillen et al. 2012).

Figure 4.2: Definition of a cavity lifetime for a wetted surface, (Hillen et al., 2012). ___ 46

Figure 4.3: Definition of crown height and bottom radius. ___ 48

Figure 4.4: A basic overview of the Matlab image processing used to determine the crown height and bottom crown radius. The order of the sequence is left to right from the top (Hillen et al., 2012). ___ 49

Figure 4.5: Schematic of the configuration utilized to view the impingement process from beneath the free surface (Hillen and Kuhlman, 2013b).

Figure 4.6: Example images of the measured sub-cavity radius. a) Side-view image of measured sub-cavity radius (red line indicates measured diameter). b) Bottom-view image of measured sub-cavity radius (red circle indicates measured diameter) (Hillen et al. 2013).

Figure 4.7: A basic overview for the side-view video image processing. From left to right: original image, binary conversion, filled cavity of the Canny edge detection outline, and the final measured bottom cavity diameter.

Figure 4.8: A basic overview for the bottom-view video image processing. From left to right: original image, filled binary conversion with glare spots connected, isolated cavity, and the final measured bottom cavity diameter.

Figure 4.9: Schematic representation of the radial cavity traverse locations for the CHR optical probe (Hillen et al. 2013).

Figure 4.10: Example determining the CHR optical probe measurement center point in the videos with the probe positioned at $R_{c}=0 \mathrm{~mm}$. The red box highlights the $1.27 \mathrm{~cm}$ diameter acrylic hole "viewport" and the red dashed line represents the probe's center point.

Figure 4.11: A basic overview of the single drop image processing. From left to right: original image, binary conversion, isolated filled drop, and the final measured drop diameter. ___ 56

Figure 5.1: Filtered and interpolated sub-cavity film thickness history compared with the original $(\mathrm{We}=621, \mathrm{Re}=$ 2,640, $\mathrm{Fr}=415, h_{0}{ }^{*}=0.5$, and $R_{c}=6.41 \mathrm{~mm}$ ). 
Figure 5.2: Interpolated and filtered sub-cavity film thickness vs. time and mapped cavity $(W e=621, \operatorname{Re}=2,640, \mathrm{Fr}=$ 415, and $h_{0}{ }^{*}=0.5$ ). a) Centerline thickness history. b) Sub-cavity thickness surface plot (Hillen et al. 2013). 61

Figure 5.3: Example of the iterative process of the sub-cavity volume trends vs. time $(W e=621, \operatorname{Re}=2,640, \mathrm{Fr}=415$, and $\left.h_{0}{ }^{*}=0.5\right)$. a) Initial constraints of the averaged and 2.5 times the minimum $h . b$ ) Final constraints (approximately 1.2 - 1.4 times the minimum $h$ ) (Hillen et al. 2013). 62

Figure 5.4: Cropped cavity surface plots (We $=621, \mathrm{Re}=2,640, \mathrm{Fr}=415$, and $\left.h_{0}{ }^{*}=0.5\right)$. a) Initial lower limit constraint. b) Initial upper limit constraint. c) Final iterated lower limit constraint. d) Final iterated upper limit constraint (Hillen et al. 2013). 63

Figure 5.5: Estimated global minimum sub-cavity film thickness versus time. ___ 64

Figure 5.6: Sub-cavity volumes computed from the optimized numerical integration method compared with estimates using the averaged $h_{\min }$ and $R_{c}$ (Hillen et al., 2013). 64

Figure 6.1: Spray droplet diameter, speed, axial velocity, and radial velocity for a 4.14 bar and $54.5 \mathrm{~L} / \mathrm{hr}$ at $z=3.81$ $\mathrm{cm}$. a) Center of the spray, $R_{s}=0 \mathrm{~cm}$. b) Edge of spray, $R_{s}=2.47 \mathrm{~cm}$.

Figure 6.2: Droplet diameter profiles at a constant $z=3.81 \mathrm{~cm}$ for the different pressure cases with uncertainty bars. a) Arithmetic mean diameter. b) Sauter mean diameter. $\triangle 1.38 \mathrm{bar}, 29.5 \mathrm{~L} / \mathrm{hr} ; \bigcirc 2.76 \mathrm{bar}, 43.5 \mathrm{~L} / \mathrm{hr}$; $\checkmark 4.14$ bar, $54.5 \mathrm{~L} / \mathrm{hr} ;-x$-plane data; ---- y-plane (Hillen et al., 2013).

Figure 6.3: Spray flow condition effects on the mean spray velocity components at $z=3.81 \mathrm{~cm}$ with uncertainty bars. $a$ ) Mean axial velocity profile. b) Mean radial velocity profile (Hillen et al., 2013).

Figure 6.4: A sketch of the PDPA probe volume at equal $R_{s}$ locations on the $x$ and $y$-axis to demonstrate the increased interference of the $x$-traverse due to the spray density.

Figure 6.5: Example of the correlation coefficient versus the sample index for the axial velocity for a flow condition of 4.14 bar and $54.5 \mathrm{~L} / \mathrm{hr}$ at $R_{s}=0 \mathrm{~cm}$ and $\mathrm{z}=3.81 \mathrm{~cm}$.

Figure 6.6: Variation of the standoff distance of the measuring profile to the nozzle for a spray flow condition of 4.14 bar and $54.5 \mathrm{~L} / \mathrm{hr}$. a) Arithmetic mean diameters. b) Sauter mean diameters. c) Average axial velocities. d) Average radial velocities.

Figure 6.7: Comparison of the spray droplet characteristics with $(z=3.60)$ and without $(z=3.81)$ the impact surface installed for 1.38 bar, 2.76 bar, 4.14 bar. a) Arithmetic mean diameter. b) Sauter mean diameter. c) Mean axial velocity. d) Mean radial velocity. $\mathbf{-} 1.38 \mathrm{bar}, 29.5 \mathrm{~L} / \mathrm{hr}$, with impact surface; - 2.76 bar, 43.5 L/hr, with impact surface; 4.14 bar, $54.5 \mathrm{~L} / \mathrm{hr}$, with impact surface; - - -1.38 bar, $29.5 \mathrm{~L} / \mathrm{hr}$, without impact surface; - - - 2.76 bar, $43.5 \mathrm{~L} / \mathrm{hr}$, without impact surface; - $\checkmark-4.14$ bar, $54.5 \mathrm{~L} / \mathrm{hr}$ without impact surface.

Figure 6.8: Droplet sizes of the spray, ejected, return, and absolute splash data with the impact surface installed for a flow condition of 4.14 bar, $54.5 \mathrm{~L} / \mathrm{hr}$ at a constant $z=3.65 \mathrm{~cm}$. a) Arithmetic mean diameter. b) Sauter mean diameter.

Figure 6.9: Droplet velocities of the spray, ejected, return, and absolute splash data with the impact surface installed for 4.14 bar, $54.5 \mathrm{~L} / \mathrm{hr}$ at a constant $z=3.65 \mathrm{~cm}$. a) Mean axial velocity, b) Mean radial velocity. $\checkmark$ Incoming spray droplets, $\triangle-$ ejected splashed droplets, $\square-$ returning splashed droplets, - absolute splashed droplets.

Figure 6.10: Absolute splash droplet characteristics at varying pressures at a constant $z=36.5$ mm. a) Arithmetic mean droplet diameter. b) Sauter mean droplet diameter. c) Mean axial velocity. d) Mean radial velocity. 89

Figure 6.11: Vertical PDPA results of the splashed droplets at $R_{s}= \pm 2.68 \mathrm{~cm}$. a) Arithmetic mean diameter, $\left.b\right)$ Sauter mean diameter, c) Mean axial velocity, d) Mean radial velocity.

Figure 7.1: Sequence of video images for (left to right) initial drop impact, initial crown formation, maximum crown height, and crown collapse for Case A (top) and Case B (bottom) (Hillen et al., 2012)

Figure 7.2: Dimensionless bottom crown radius histories for five runs. a) Case A: We =141, $\operatorname{Re}=5,700, \mathrm{Fr}=103$, and $\left.h_{0}{ }^{*}=0.614 . b\right)$ Case B: $W e=633, \operatorname{Re}=17,100, \mathrm{Fr}=115$, and $h_{0}{ }^{*}=0.113$ (Hillen et al. 2012). _ 100

Figure 7.3: Dimensionless crown height histories for five runs. a) Case A: We $=141, \operatorname{Re}=5,700, \mathrm{Fr}=103$, and $\mathrm{h}_{0}{ }^{*}=$ 0.614. b) Case B: We $=633, \operatorname{Re}=17,100, \mathrm{Fr}=115$, and $h_{0}{ }^{*}=0.113$ (Hillen et al. 2012).___ 101

Figure 7.4: Centerline sub-cavity liquid film thickness histories for five runs. a) Case A: We =141, Re = 5,700, Fr = 103, and $h_{0}{ }^{*}=0.614$. b) Case B: We $=633, \operatorname{Re}=17,100, \mathrm{Fr}=115$, and $h_{0}{ }^{*}=0.113$ (Hillen et al. 2012).

102

Figure 7.5: Sub-cavity history carpet. a) Case $A:$ We $=141, \operatorname{Re}=5,700, F r=103$, and $\left.h_{0}{ }^{*}=0.614 . b\right)$ Case B: We $=$ 633, $\operatorname{Re}=17,100, \mathrm{Fr}=115$, and $h_{0}{ }^{*}=0.113$ (Hillen et al. 2012). 103 
Figure 7.6: Comparison of the bottom crown radius histories from the experiment and $2 D$ simulation. a) Case A: We $=$ 141, $\operatorname{Re}=5,700, \mathrm{Fr}=103$, and $h_{0}{ }^{*}=0.614$. b) Case B: We $=633, \operatorname{Re}=17,100, \mathrm{Fr}=115$, and $h_{0}{ }^{*}=$ 0.113 (Hillen et al. 2012).

104

Figure 7.7: Comparison of the centerline sub-cavity liquid film thickness histories from the experiment and $2 D$ simulation. a) Case A: We =141, $\operatorname{Re}=5,700, \mathrm{Fr}=103$, and $h_{0}{ }^{*}=0.614$. b) Case B: We $=633, \operatorname{Re}=$ $17,100, \mathrm{Fr}=115$, and $h_{0}{ }^{*}=0.113$ (Hillen et al. 2012). 105

Figure 7.8: Sub-cavity radius histories varying $h_{0}{ }^{*}$ for Case $3(\mathrm{We}=621$, $\operatorname{Re}=3,090, \mathrm{Fr}=415)$. a) Bottom-view measurements. b) Side-view measurements. 107

Figure 7.9: Sub-cavity radius histories varying We at a constant $h_{0}{ }^{*}=0.5$. a) Bottom-view measurements. b) Side-view measurements. 108

Figure 7.10: Sample contour plot of with the bottom-view (dashed line), side-view (dotted line), and averaged (solid line) sub-cavity radius overlaid for Case 3, $h_{0}{ }^{*}=0.5(\mathrm{We}=621, \mathrm{Re}=3,090, \mathrm{Fr}=415)$.

Figure 7.11: Images taken from high-speed videos showing the cavity formation over time at an $h_{0}{ }^{*}=0.5$ for Cases 1,3 , and 5 test conditions. The red dashed line is the true free surface interface. a) We $=146, \operatorname{Re}=1,290, \mathrm{Fr}$ =99.0. b) $W e=621, \operatorname{Re}=2,640, \mathrm{Fr}=415$. c) $\mathrm{We}=984, \mathrm{Re}=3,560, \mathrm{Fr}=499$ (Hillen et al. 2013).111

Figure 7.12: High-speed video images showing the crown and cavity formation over time at varying $h_{0}{ }^{*}$ for Cases 3 and 5. The red dashed line is the true free surface interface. a) $W e=145, \operatorname{Re}=1,190, \mathrm{Fr}=100.0 . \mathrm{b}) \mathrm{We}=$ 135, $\operatorname{Re}=1,180, \mathrm{Fr}=94$. c) $W e=993, \operatorname{Re}=3,570, \mathrm{Fr}=510 . \mathrm{d}) \mathrm{We}=984, \operatorname{Re}=3,560, \mathrm{Fr}=407$ (Hillen et al., 2013)

112

Figure 7.13: Examination of cavity wave denoted as a capillary wave in literature. Case 3: We $=145$, Re $=1,190, F r=$ 100.0, and $h_{0}{ }^{*}=1.0$. The red dashed line is the true free surface interface and the red arrow highlights the cavity wave.

Figure 7.14: Example of unique prompt splash for Case 4, $h_{0}{ }^{*}=0.9(\mathrm{We}=762, \mathrm{Re}=3,080, \mathrm{Fr}=398) . \mathrm{The}$ initial splash is highlighted by the dashed ellipse. ___ 115

Figure 7.15: The effects of varying the $h_{0}{ }^{*}$ and We on the centerline dimensionless film thickness. a) Varying $h_{0}{ }^{*}$ for a constant $W e \approx 640, \operatorname{Re} \approx 2,700, \mathrm{Fr} \approx 430$ of Case 3 for. b) Varying the We at a constant $h_{0}{ }^{*}=0.5$ (Hillen et al., 2013).

117

Figure 7.16: The effects of varying the We and $h_{0}{ }^{*}$ on the centerline film thickness ratio $h / h_{0}$. a) Varying $h_{0}{ }^{*}$ for a constant $W e \approx 640, \operatorname{Re} \approx 2,700, \mathrm{Fr} \approx 430$ of Case 3. b) Varying the We at a constant $h_{0}{ }^{*}=0.5$ (Hillen et al., 2013). 118

Figure 7.17: Sub-cavity liquid film thickness surface plots for Cases 1 - 5 at a fixed 0 - 250 um contour color scale. Note for cases 4 and $5 h_{0}{ }^{*}=0.9$ in the left hand column. 121

Figure 7.18: Effects of varying the $h_{0}{ }^{*}$ on the dimensionless sub-cavity volume histories at a constant We $=637$, Re $=$ 2,686, Fr = 432 of Case 3 (Hillen et al., 2013). 125

Figure 7.19: Effects of varying We on the dimensionless sub-cavity volume histories. a) Constant $h_{0}{ }^{*}=1.0\left(h_{0}{ }^{*}=0.9\right.$ for cases 4 and 5). b) Constant $h_{0}{ }^{*}=0.5$. c) Constant $h_{0}{ }^{*}=0.2$.

Figure 7.20: Effects of varying the $h_{0}{ }^{*}$ on the dimensionless sub-cavity volume histories at a constant We $=142$, Re $=$ 1,220, Fr $=98.0$ of Case 1 (Hillen et al., 2013).

Figure A.1: Base mounting plate (0.25 in thick stainless steel sheet) used to attach to the leveling plate to the structural frame (units in inches).

Figure A.2: Leveling plate (0.25 in thick stainless steel sheet) used to level the single droplet tank (units in inches). 10

Figure A.3: Base plate (0.25 in thick stainless steel sheet) used to attach the acrylic tank to the micro-traverse (units in inches).

141

Figure A.4: Acrylic impact stand used for sub-cavity liquid film thickness experiments (units in inches). ___ 141

A.5: Acrylic impact stand with larger viewing port used for the BV sub-cavity radius experiments (units in inches). 142 Figure A.6: Acrylic tank (units in inches). 142

Figure A.7: Final Single drop test facility Assembly. a) Backlit configuration. b) Tank and impact surface fixed to the micro-traverse.

Figure B.1: Spray droplet diameter, speed, axial velocity, and radial velocity for a 2.76 bar and $43.5 \mathrm{~L} / \mathrm{hr}$ at $z=3.81$ $\mathrm{cm}$. a) Center of the spray, $\left.R_{s}=0 \mathrm{~cm} . b\right)$ Edge of spray, $R_{s}=2.47 \mathrm{~cm}$.

Figure B.2: Spray droplet diameter, speed, axial velocity, and radial velocity for a 1.38 bar and $29.5 \mathrm{~L} / \mathrm{hr}$ at $z=3.81$ $\mathrm{cm}$. a) Center of the spray, $R_{s}=0 \mathrm{~cm}$. b) Edge of spray, $R_{s}=2.47 \mathrm{~cm}$.

Figure B.3: Average droplet velocity magnitude profiles at a constant $z=3.81 \mathrm{~cm}$ for the different pressure cases $\mathbf{\Delta} \Delta$ $1.38 \mathrm{bar}, 29.5 \mathrm{~L} / \mathrm{hr} ; \bigcirc 2.76 \mathrm{bar}, 43.5 \mathrm{~L} / \mathrm{hr} ; \diamond 4.14 \mathrm{bar}, 54.5 \mathrm{~L} / \mathrm{hr} ;-x$-plane data; ---- y-plane. 
Figure B.4: Variation of the standoff distance of the measuring profile to the nozzle for a spray flow condition of the average velocity magnitude for 4.14 bar and $54.5 \mathrm{~L} / \mathrm{hr}$.

Figure B.5: Average droplet characteristic profiles at a constant $z=3.18 \mathrm{~cm}$ for the different pressure cases. a) Arithmetic mean diameter. b) Sauter mean diameter. c) Average axial velocity. d) Average radial velocity. e) Average velocity magnitude. $\Delta 1.38 \mathrm{bar}, 29.5 \mathrm{~L} / \mathrm{hr} ; 02.76 \mathrm{bar}, 43.5 \mathrm{~L} / \mathrm{hr}$; $4.14 \mathrm{bar}, 54.5 \mathrm{~L} / \mathrm{hr}$.

Figure B.6: Average droplet characteristic profiles at a constant $z=4.45 \mathrm{~cm}$ for the different pressure cases. a) Arithmetic mean diameter. b) Sauter mean diameter. c) Average axial velocity. d) Average radial velocity. e) Average velocity magnitude. $\mathbf{\Delta} 1.38 \mathrm{bar}, 29.5 \mathrm{~L} / \mathrm{hr}$; $2.76 \mathrm{bar}, 43.5 \mathrm{~L} / \mathrm{hr}$; $4.14 \mathrm{bar}, 54.5 \mathrm{~L} / \mathrm{hr}$.

Figure C.1: Histograms of the incoming spray droplet data for 4.14 bar, $54.5 \mathrm{~L} / \mathrm{hr}$ at $z=3.65 \mathrm{~cm}$ at a single point. a) $R_{s}$ $=0 \mathrm{~cm} . \mathrm{b}) R_{s}=2.47 \mathrm{~cm}$. 151

Figure C.2: Histograms of the ejected splashed droplet data for $4.14 \mathrm{bar}, 54.5 \mathrm{~L} / \mathrm{hr}$ at $\mathrm{z}=3.65 \mathrm{~cm}$ at a single point. a) $\left.R_{s}=0 \mathrm{~cm} . b\right) R_{s}=2.47 \mathrm{~cm}$.

Figure C.3: Histograms of the returning splashed droplet data for $4.14 \mathrm{bar}, 54.5 \mathrm{~L} / \mathrm{hr}$ at $\mathrm{z}=3.65 \mathrm{~cm}$ at a single point. a) $R_{s}=0 \mathrm{~cm}$. b) $R_{s}=2.47 \mathrm{~cm}$.

Figure C.4: Histograms of the absolute splashed droplet data for $4.14 \mathrm{bar}, 54.5 \mathrm{~L} / \mathrm{hr}$ at $\mathrm{z}=3.65 \mathrm{~cm}$ at a single point. a) $\left.R_{s}=0 \mathrm{~cm} . \mathrm{b}\right) R_{s}=2.47 \mathrm{~cm}$.

Figure C.5: Histograms of the incoming spray droplet data for $2.76 \mathrm{bar}, 43.5 \mathrm{~L} / \mathrm{hr}$ at $\mathrm{z}=3.65 \mathrm{~cm}$ at a single point. a) $R_{s}$ $=0 \mathrm{~cm} . \mathrm{b}) R_{s}=2.47 \mathrm{~cm}$.

Figure C.6: Histograms of the ejected splashed droplet data for $2.76 \mathrm{bar}, 43.5 \mathrm{~L} / \mathrm{hr}$ at $\mathrm{z}=3.65 \mathrm{~cm}$ at a single point. a) $\left.R_{s}=0 \mathrm{~cm} . \mathrm{b}\right) R_{s}=2.47 \mathrm{~cm}$. 156

Figure C.7: Histograms of the returning splashed droplet data for $2.76 \mathrm{bar}, 43.5 \mathrm{~L} / \mathrm{hr}$ at $\mathrm{z}=3.65 \mathrm{~cm}$ at a single point. a) $R_{s}=0 \mathrm{~cm}$. b) $R_{s}=2.47 \mathrm{~cm}$.

Figure C.8: Histograms of the absolute splashed droplet data for $2.76 \mathrm{bar}, 43.5 \mathrm{~L} / \mathrm{hr}$ at $\mathrm{z}=3.65 \mathrm{~cm}$ at a single point. a) $\left.R_{s}=0 \mathrm{~cm} . \mathrm{b}\right) R_{s}=2.47 \mathrm{~cm}$. 158

Figure C.9: Histograms of the incoming spray droplet data for 1.38 bar, $29.5 \mathrm{~L} / \mathrm{hr}$ at $z=3.65 \mathrm{~cm}$ at a single point. a) $R_{s}$ $=0 \mathrm{~cm} . \mathrm{b}) R_{s}=2.47 \mathrm{~cm}$. 159

Figure C.10: Histograms of the ejected splashed droplet data for $1.38 \mathrm{bar}, 29.5 \mathrm{~L} / \mathrm{hr}$ at $\mathrm{z}=3.65 \mathrm{~cm}$ at a single point. a) $\left.R_{s}=0 \mathrm{~cm} . b\right) R_{s}=2.47 \mathrm{~cm}$. 160

Figure C.11: Histograms of the returning splashed droplet data for $1.38 \mathrm{bar}, 29.5 \mathrm{~L} / \mathrm{hr}$ at $\mathrm{z}=3.65 \mathrm{~cm}$ at a single point . a) $R_{s}=0 \mathrm{~cm}$. b) $R_{s}=2.47 \mathrm{~cm}$. 161

Figure C.12: Histograms of the absolute splashed droplet data for $1.38 \mathrm{bar}, 29.5 \mathrm{~L} / \mathrm{hr}$ at $\mathrm{z}=3.65 \mathrm{~cm}$ at a single point. a) $\left.R_{s}=0 \mathrm{~cm} . b\right) R_{s}=2.47 \mathrm{~cm}$. 162

Figure C.13: Droplet velocity magnitude of the spray, ejected, return, and absolute splash data with the impact surface installed for a flow condition of $4.14 \mathrm{bar}, 54.5 \mathrm{~L} / \mathrm{hr}$ at a constant $\mathrm{z}=36.5 \mathrm{~mm}$. $\checkmark$ Impinging spray droplets, $\triangle \leftarrow$ ejected splashed droplets, $-\square-$ returning splashed droplets, $-\mathbf{-}$ absolute splashed droplets. 163

Figure C.14: Absolute splashed droplet mean velocity magnitude profile for varying pressures at a constant $z=36.5$ $\mathrm{mm}$. 163

Figure C.15: PDPA data of the mean velocity magnitude versus $z$ at $R_{s}= \pm 2.68 \mathrm{~cm}$. 163

Figure C.16: Comparison of the spray droplet characteristics with and without the impact surface installed a flow condition of $2.76 \mathrm{bar}, 43.5 \mathrm{~L} / \mathrm{hr}$ at a constant $z=36.5 \mathrm{~mm}$. a) Arithmetic mean diameter. b) Sauter mean diameter. c) Mean axial velocity. d) Mean radial velocity. e) Mean velocity magnitude. $\checkmark$ Impinging spray droplets, $\triangle-$ ejected splashed droplets, $\square-\square$ returning splashed droplets, $-\mathbf{-}$ absolute splashed droplets.

164

Figure C.17: Comparison of the spray droplet characteristics with and without the impact surface installed a flow condition of $1.38 \mathrm{bar}, 29.5 \mathrm{~L} / \mathrm{hr}$ at a constant $z=36.5 \mathrm{~mm}$. a) Arithmetic mean diameter. b) Sauter mean diameter. c) Mean axial velocity. d) Mean radial velocity. e) Mean velocity magnitude. $\checkmark$ Impinging spray droplets, $\neg-$ ejected splashed droplets, $\square-\square$ returning splashed droplets, $-\mathbf{-}$ absolute splashed droplets. 165

Figure C.18: Ejected splashed droplet characteristics at varying pressures at a constant $z=36.5 \mathrm{~mm}$. a) Arithmetic mean droplet diameter. b) Sauter mean droplet diameter. c) Mean axial velocity. d) Mean radial velocity. e) Mean velocity magnitude. 166 
Figure C.19: Returning splashed droplet characteristics at varying pressures at a constant $z=36.5$ mm. a) Arithmetic mean droplet diameter. b) Sauter mean droplet diameter. c) Mean axial velocity. d) Mean radial velocity. e) Mean velocity magnitude. 167

Figure D.1: Surface tension versus glycerol-water solution percent concentration by mass for 169

Figure D.2: Viscosity prediction from Cheng (2008) compared with data from Glycerol Producer's Association (1963). 170

Figure D.3: Density prediction from Cheng (2008) compared with data from Glycerol Producer's Association (1963).

Figure D.4: Comparison of the predictive capability of curve fits from Glycerol Producers Association (1963) with available refractive indices of glycerol-water mixtures. 171

Figure D.5: Initial film thickness manual measurements. 171

Figure E.1: Sub-cavity radius histories varying for Cases $1-5$ at $h_{0}{ }^{*}=1.0\left(h_{0}{ }^{*}=0.9\right.$ for Cases 4 and 5). a) Bottomview results. b) Side-view results. 175

Figure E.2: Sub-cavity radius histories varying for Cases $1-5$ at $h_{0}{ }^{*}=0.2$. a) Bottom-view results. $\left.b\right)$ Side-view results. 175

Figure E.3: Contour plot of with the bottom-view (dashed line), side-view (dotted line), and averaged (solid line) subcavity radius overlaid for Case $1, h_{0}{ }^{*}=0.2(\mathrm{We}=135, \mathrm{Re}=1,180, \mathrm{Fr}=94.0)$. 176

Figure E.4: Contour plot of with the bottom-view (dashed line), side-view (dotted line), and averaged (solid line) subcavity radius overlaid for Case $1, h_{0}{ }^{*}=0.5(\mathrm{We}=146, \mathrm{Re}=1,290, \mathrm{Fr}=99.0)$.___ 176

Figure E.5: Contour plot of with the bottom-view (dashed line), side-view (dotted line), and averaged (solid line) subcavity radius overlaid for Case $1, h_{0}{ }^{*}=1.0($ We $=145, \operatorname{Re}=1,190, \mathrm{Fr}=100)$. _ 177

Figure E.6: Contour plot of with the bottom-view (dashed line), side-view (dotted line), and averaged (solid line) subcavity radius overlaid for Case $2, h_{0}{ }^{*}=0.2$ ( We $=414, \operatorname{Re}=2,290, \mathrm{Fr}=217$ ). 177

Figure E.7: Contour plot of with the bottom-view (dashed line), side-view (dotted line), and averaged (solid line) subcavity radius overlaid for Case $2, h_{0}{ }^{*}=0.5(\mathrm{We}=413, \mathrm{Re}=2,270, \mathrm{Fr}=219)$ ___ 178

Figure E.8: Contour plot of with the bottom-view (dashed line), side-view (dotted line), and averaged (solid line) subcavity radius overlaid for Case $2, h_{0}{ }^{*}=1.0(\mathrm{We}=410, \mathrm{Re}=2,250, \mathrm{Fr}=217)$ ___ 178

Figure E.9: Contour plot of with the bottom-view (dashed line), side-view (dotted line), and averaged (solid line) subcavity radius overlaid for Case $3, h_{0}{ }^{*}=0.2(\mathrm{We}=667, \mathrm{Re}=2,740, \mathrm{Fr}=439)$. 179

Figure E.10: Contour plot of with the bottom-view (dashed line), side-view (dotted line), and averaged (solid line) subcavity radius overlaid for Case 3, $h_{0}{ }^{*}=1.0(\mathrm{We}=621, \mathrm{Re}=2,640, \mathrm{Fr}=415)$.

Figure E.11: Contour plot of with the bottom-view (dashed line), side-view (dotted line), and averaged (solid line) subcavity radius overlaid for Case $4, h_{0}{ }^{*}=0.2(\mathrm{We}=780, \mathrm{Re}=3,100, \mathrm{Fr}=413)$ _ 180

Figure E.12: Contour plot of with the bottom-view (dashed line), side-view (dotted line), and averaged (solid line) subcavity radius overlaid for Case $4, h_{0}{ }^{*}=0.5$ (We $\left.=771, \mathrm{Re}=3,090, \mathrm{Fr}=407\right)$. 180

Figure E.13: Contour plot of with the bottom-view (dashed line), side-view (dotted line), and averaged (solid line) subcavity radius overlaid for Case $4, h_{0}{ }^{*}=0.9(\mathrm{We}=762, \mathrm{Re}=3,080, \mathrm{Fr}=398)$.

Figure E.14: Contour plot of with the bottom-view (dashed line), side-view (dotted line), and averaged (solid line) subcavity radius overlaid for Case $5, h_{0}{ }^{*}=0.2(\mathrm{We}=984, \mathrm{Re}=3,560, \mathrm{Fr}=407)$.

Figure E.15: Contour plot of with the bottom-view (dashed line), side-view (dotted line), and averaged (solid line) subcavity radius overlaid for Case $5, h_{0}{ }^{*}=0.5(\mathrm{We}=984, \mathrm{Re}=3,560, \mathrm{Fr}=499)$. _ 182

Figure E.16: Contour plot of with the bottom-view (dashed line), side-view (dotted line), and averaged (solid line) subcavity radius overlaid for Case $5, h_{0}{ }^{*}=0.9(\mathrm{We}=993, \mathrm{Re}=3,570, \mathrm{Fr}=510)$. 182

Figure E.17: Dimensionless sub-cavity liquid film thickness $\left(h^{*}\right)$ histories for varying the initial droplet condition (We, Re, and $\mathrm{Fr}$ ) at a constant $h_{0}{ }^{*}$. a) $h_{0}{ }^{*}=1.0$ for Case $1-3$ and $h_{0}{ }^{*}=0.9$ for Cases $\left.4-5 . b\right) h_{0}{ }^{*}=0.2 .183$

Figure E.18: Sub-cavity liquid film thickness ratio $h / h_{0}$ histories for varying the initial droplet condition (We, Re, and Fr) at a constant $h_{0}{ }^{*}$ a) $h_{0}{ }^{*}=1.0$ for Case $1-3$ and $h_{0}{ }^{*}=0.9$ for Cases $\left.4-5 . b\right) h_{0}{ }^{*}=0.2$. 183

Figure E.19: Dimensionless sub-cavity liquid film thickness $\left(h^{*}\right)$ histories for varying the $h_{0}{ }^{*}$ at a constant initial droplet condition (We, Re, and Fr). a) Case 1. b) Case 2. c) Case 3. d) Case 4. 184

Figure E.20: Sub-cavity liquid film thickness ratio $h / h_{0}$ histories for varying the $h_{0}{ }^{*}$ at a constant initial droplet condition (We, Re, and Fr). a) Case 1. b) Case 2. c) Case 3. d) Case 4. 185

\section{Page xii}




\section{TABLES}

Table 1.1: Single train droplet frequency, diameter, spreading area, and maximum heat flux for experiments by Soriano

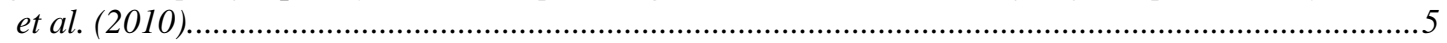

Table 2.1: Summary of Monte-Carlo simulation time scales for a drop with a $D_{32}=48 \mu \mathrm{m}, \mathrm{V}=12 \mathrm{~m} / \mathrm{s}, \mathrm{FC}-72 \mathrm{at} \mathrm{a}$ flow

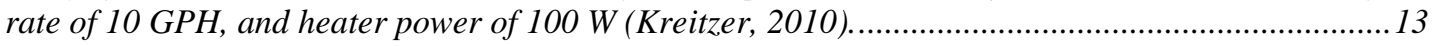

Table 2.2: Summary of various deposition/rebound/splash criteria reported in literature. .......................................19

Table 2.3: Summary of measured liquid film thickness created by an impinging spray and spray droplet sizes studied in

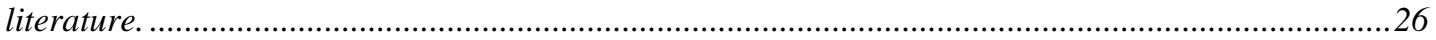

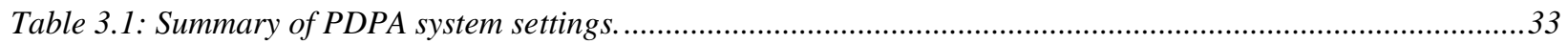

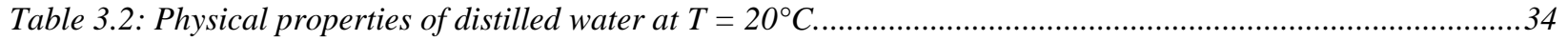

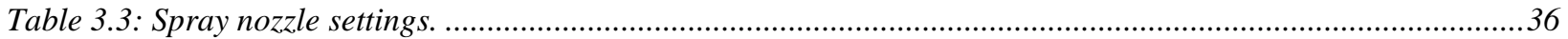

Table 4.1: Dimensional and corresponding dimensionless parameters for preliminary single drop cavity investigation

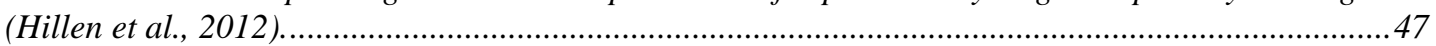

Table 6.1: Average, maximum, and minimum PDPA probe volume uncertainty between the $x$ and y-traverse profiles of the arithmetic and Sauter mean diameter for varying pressures at a constant $z=3.81 \mathrm{~mm} \ldots \ldots \ldots \ldots . . . .73$

Table 6.2: Average, maximum, and minimum PDPA probe volume uncertainty between the $x$ and y-axis measurements of the mean axial velocity for varying pressures at a constant $z=3.81 \mathrm{~mm}$.................................73

Table 6.3: The total measurement sample and the decorrelation sample index of the droplet diameter, axial velocity, and radial velocity at $z=3.81 \mathrm{~cm}$ for the $x$ and $y$-traverses $\left(\right.$ no $n_{\text {dec }}\left(v_{r}\right)$ for the $y$-traverse)...................75

Table 6.4: The standard deviation of the droplet diameter, axial velocity, and radial velocity at $z=3.81 \mathrm{~cm}$ for the $x$ and

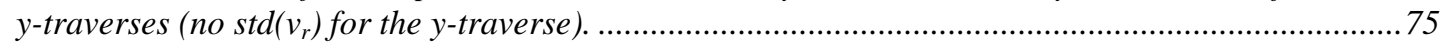

Table 6.5: The statistical fluctuation uncertainty of the droplet diameter, axial velocity, and radial velocity at $z=3.81$

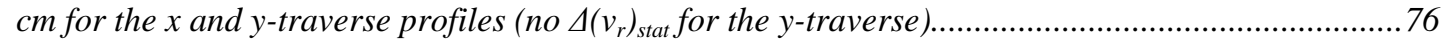

Table 6.6: The total uncertainty of the droplet diameter, axial velocity, and radial velocity at .................................77

Table 6.7: Spatial standard deviation of $D_{32}$ across $0 \leq R_{s} \leq 2.68 \mathrm{~cm}$ for varying flow conditions at $z=3.81 \mathrm{~cm} . \ldots 78$

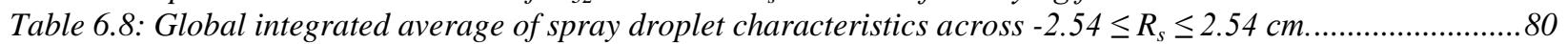

Table 6.9: Characterization of the spray in terms of the average We, Re, and Fr for each spray condition. Dimensionless numbers were computed using the axial velocity component. ..........................................................8 81

Table 6.10: Mean drop characteristics calculated from the 5\% population consisting of the smallest droplets at $z=3.81$

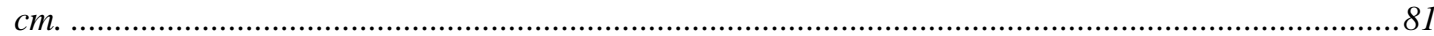

Table 6.11: Dimensionless numbers calculated from the $5 \%$ population consisting of the smallest droplets at a constant $z=3.81 \mathrm{~cm}$. .82

Table 6.12: Mean drop characteristics calculated from the 5\% population consisting of the largest droplets at a constant $z=3.81 \mathrm{~cm}$.

Table 6.13: Average dimensionless numbers calculated from the 5\% population consisting of the largest droplets at a

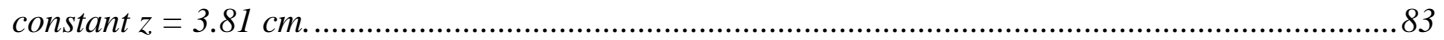

Table 6.14: The averaged standard deviation of the splash droplet characteristics averaged across $-2.54<R_{s}<2.54 \mathrm{~cm}$

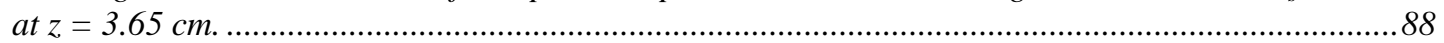

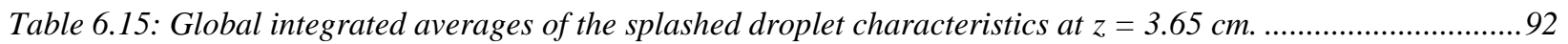

Table 6.16: Dimensionless numbers computed from the global average characteristics of the ejected, return, and absolute splashed droplets at $z=3.65 \mathrm{~cm}$........................................................................93

Table 6.17: Single drop experiment dimensionless droplet parameters (Hillen et al., 2013)...............................94

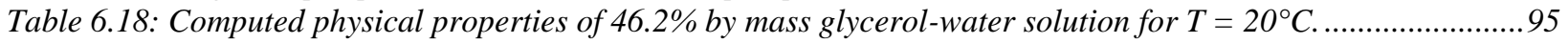

Table 7.1: Dimensionless parameters measured in the experiments and their respective percent error to the target

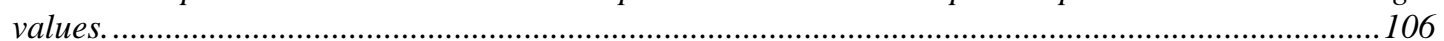

Table 7.2: Computed splashing limits based on Cossali et al. (1997) compared with experimental observations...114 Table 7.3: Nominal absolute minimum, absolute maximum, and mean liquid sub-cavity film thickness measured in the sub-cavity region. Also included is the duration of the thin liquid sub-cavity film thickness and maximum sub-cavity radius. Results are for Cases $1-5$ at $h_{0}{ }^{*}=1.0,0.5$, and 0.2 . 123

Table D.1: Target liquid film thickness measured by the CHR sensor and by video. Included is the refractive index calculated by the video analysis for a glycerol-water mixture of $46.2 \%$........................................172

Table E.1: Measured sub-cavity single drop parameters and standard deviations for Cases $1-5$ for each $h_{0}{ }^{*} \ldots . . . .174$ Table E.2: Average ambient conditions and respective variance recorded during the Single drop experiments for Cases $1-5$ for each $h_{0}{ }^{*}$ 174 


\section{NOMENCLATURE}

\section{Latin Symbols}

\begin{tabular}{|c|c|c|}
\hline$A$ & $=$ & Area \\
\hline$c_{v}$ & $=$ & Coefficient of variation \\
\hline$C_{m}$ & $=$ & Mass fraction \\
\hline$C_{p}$ & $=$ & Specific heat \\
\hline$d$ & $=$ & Droplet diameter \\
\hline$D$ & $=$ & Arithmetic mean droplet diameter \\
\hline$D_{32}$ & $=$ & Sauter mean droplet diameter \\
\hline$h_{f g}$ & $=$ & Latent heat \\
\hline$g$ & $=$ & Gravity \\
\hline$f$ & $=$ & Frequency \\
\hline$F r$ & $=$ & Froude number \\
\hline$h$ & $=$ & Liquid film thickness \\
\hline$H_{\text {crown }}$ & $=$ & Crown height \\
\hline$K$ & $=$ & Splashing criteria for a dry surface \\
\hline$m$ & $=$ & Mass \\
\hline$m_{c}$ & $=$ & Liquid mass flow in thin cavity film \\
\hline$N$ & $=$ & Total number of samples \\
\hline$n_{d e c}$ & $=$ & Decorrelated sample index \\
\hline$O h$ & $=$ & Ohnesorge number \\
\hline$P$ & $=$ & Pressure \\
\hline$Q$ & $=$ & Volume flow rate \\
\hline$q "$ & $=$ & Heat flux \\
\hline$R$ & $=$ & Radial location \\
\hline $\operatorname{Re}$ & $=$ & Reynolds number \\
\hline$R H$ & $=$ & Relative humidity \\
\hline std & $=$ & Standard Deviation \\
\hline$T$ & $=$ & Temperature \\
\hline$t$ & $=$ & Time \\
\hline$v$ & $=$ & Droplet velocity \\
\hline$V$ & $=$ & Arithmetic mean velocity \\
\hline Vol & $=$ & Sub-cavity volume \\
\hline$W e$ & $=$ & Weber number \\
\hline$x c$ & $=$ & Center of image from the left hand side \\
\hline$Y$ & $=$ & Splashing criteria for thin liquid layers \\
\hline$z$ & $=$ & Standoff distance from the nozzle tip \\
\hline
\end{tabular}

\section{Greek Symbols}

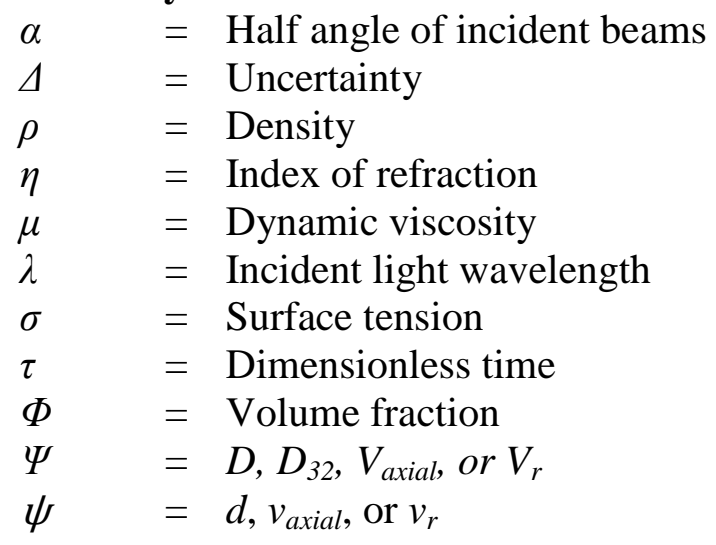

\section{Superscripts}

* $=$ Dimensionless parameter

$$
\begin{array}{ll}
\text { Subscripts } \\
0 \quad=\text { Initial value } \\
0.75 \%=\text { Instrumentation uncertainty } \\
\text { axial } & =\text { Axial velocity component } \\
B & =\text { Bottom crown } \\
c & =\text { Cavity } \\
c w & =\text { Cavity wave } \\
G & =\text { Glycerol } \\
\text { cg } & =\text { Cavity growth } \\
\text { inter } & =\text { Interaction } \\
\text { lim } & =\text { Limit } \\
\text { max } & =\text { Maximum value } \\
\text { min } & =\text { Minimum value } \\
P V & =\text { PDPA probe volume } \\
r & =\text { Radial velocity component } \\
\text { s } & =\text { Spray } \\
\text { sat } & =\text { Saturation } \\
\text { sol } & =\text { Glycerol-water solution } \\
\text { stat } & =\text { Statistical data fluctuation } \\
W & =\text { Water } \\
x & =x \text { spray traverse profile } \\
y & =y \text { spray traverse profile }
\end{array}
$$

\section{Acronyms}

$\begin{array}{ll}\text { BV } & - \text { Bottom-view } \\ \text { CFD } & - \text { Computational Fluid Dynamics } \\ \text { CHF } & - \text { Critical Heat Flux } \\ \text { CHR } & - \text { CHRocodile }\end{array}$




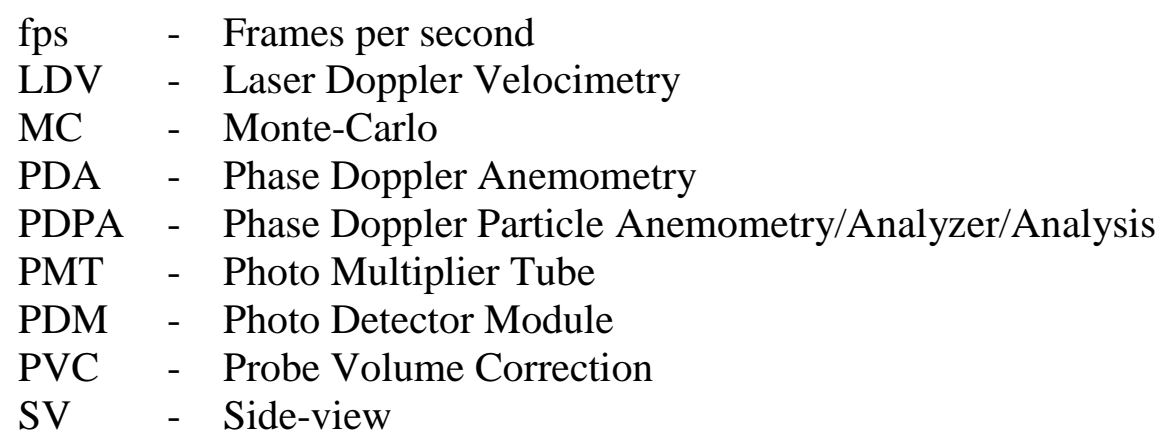




\section{CHAPTER 1: INTRODUCTION}

As technology advances the continuing trend of electronic component size reduction will result in more compact systems and denser geometries, resulting in reduced surface area-to-volume ratios. This is expected to make waste heat removal more challenging requiring active enhanced thermal management systems. Spray cooling is an attractive two phase candidate capable of uniformly removing large amounts of waste heat at relatively low surface temperatures with no temperature overshoot (Kim, 2007). Spray cooling experiments performed by Lin and Ponnappan (2003) demonstrated heat fluxes as high as $500 \mathrm{~W} / \mathrm{cm}^{2}$ before the onset of critical heat flux (CHF) using water as the coolant. They also achieved heat fluxes of $100 \mathrm{~W} / \mathrm{cm}^{2}$ at relatively low surface temperatures of $\left(T_{\text {wall }}-T_{\text {sat }}\right) \approx 12 \mathrm{~K}$ also using water as their coolant. Potential applications include, but are not limited to, small high density electronics in future space-based power systems, lidar instruments, and laser diode arrays (Silk et al., 2008).

There are two main approaches to designing spray cooling systems: either performing experiments on physical systems, or simulations using Computational Fluid Dynamics (CFD). However, issues in the required resources and time are limiting for both methods. Spray cooling experiments are costly and take considerable time to build, with limited flexibility for new iterations for existing setups. The use of CFD to fully simulate spray cooling systems would require impractical resources due to the complexity of the spray. This is true even with advancements in modern computing processes, whereas typical sprays have drop fluxes on the order of a $10^{6}$ drops $/\left(\mathrm{s} \cdot \mathrm{cm}^{2}\right)$ (Kuhlman et al., 2011). An example of the inefficiency of CFD can be found in the direct numerical simulations performed by Sarkar and Selvam (2009) of a single 3-D drop impingement (20 time steps) with $129 \times 129 \times 129$ grid sizes. They were able to reduce the computation time of a single 3-D drop impingement from 60 days to 30 hours using a multigrid 
conjugate gradient solver and 32 processors. Thus, a more efficient and feasible solution such as a phenomenological model capable of capturing the basic, relevant physics would be very desirable. Kreitzer (2010) describes one approach to such a model in the form of a Monte-Carlo (MC) simulation spray cooling model. The initial MC model developed by Kreitzer (2010) is still in development with the ultimate goal to accurately predict the heat flux for given nozzle flow conditions, for smooth heater surface geometries, and heater surface temperatures below the Leidenfrost point in reasonable computation times. Figure 1.1 shows an example image of a spray generated by a nozzle impinging normal to a $5.08 \mathrm{~cm}$ diameter unheated surface similar to that for which the final MC model will apply.

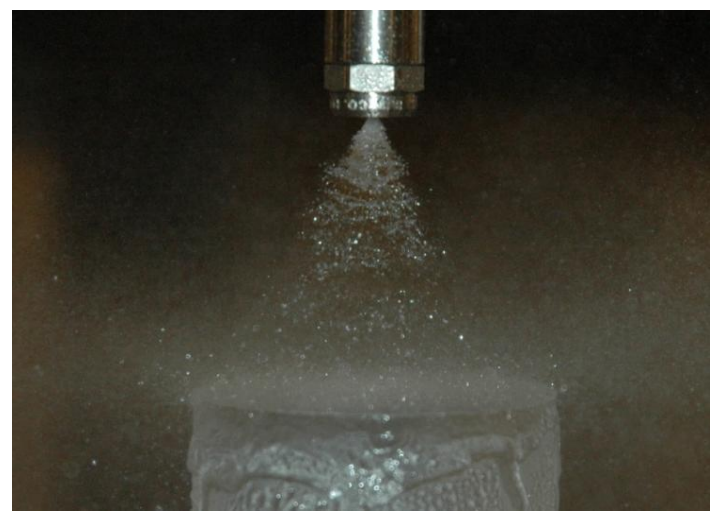

Figure 1.1: Full cone water spray generated by a Spraying Systems FullJet 1/8-G nozzle on an unheated $5.08 \mathrm{~cm}$ diameter surface (Hillen et al. 2013).

The Monte-Carlo method can be described as a statistical approach to solve differential equations and is particularly effective simulating fluid flow and heat transfer. In its basic form, random data is generated to fit a range of conditions to solve coupled equations to predict a final solution (Metropolis and Ulam, 1949). The initial MC model developed by Kreitzer (2010) incorporated empirical correlations developed from experiments and CFD simulations. A detailed summary of the initial MC model is given in Chapter 2. Although the MC model shows promise it still lacks the necessary physics to be quantitatively accurate. One important type of missing data is the lifetime and volume of the liquid beneath the cavity generated by an impinging droplet on a 
wetted surface. The main focus of the present thesis is on this liquid volume beneath the droplet impact cavity, or "sub-cavity volume" as it will be referred to herein. The importance of the subcavity volume will be discussed subsequently.

A brief overview of spray cooling is provided, but it is not the author's intent to summarize all of the available literature. The basic spray cooling process consists of a liquid coolant, typically dielectric, that is forced through a nozzle orifice generating an atomized spray. The spray delivers many small, cool droplets that directly impinge onto the heated surface, quickly establishing a thin residual liquid film. Additional fresh, cool droplets continuously impinge on the heated liquid film creating a film flow over the heater surface. The interaction of the cool droplets and liquid film on the heated surface results in high heat fluxes. The underlying physics of the heat transfer mechanisms are not fully understood due to their complexity. Therefore, spray cooling remains in the research and development stage. The number of input variables for spray cooling contributes to this complexity; these may be summarized into five main categories: the impinging spray parameters, heater geometry and surface conditions, the working fluid, ambient atmosphere conditions, and gravity (Silk et al., 2008). Extensive literature exists that examines the underlying heat transfer mechanisms, focused on liquid film dynamics on the heated surface. Topics considered include the film's liquid/vapor interface evaporation; homogenous, secondary, and heterogeneous bubble nucleation; forced convection mixing of the cool droplets and heated film liquid; and vapor bubble departures rewetting the heater surface (Horacek et al., 2004, Kim, 2007, Silk et al. 2008, and Sarkar and Selvam, 2009). All of these mechanisms contribute to the heat transfer, but which is the most important is debatable.

One potential contributing spray cooling heat transfer mechanism that is generally neglected in literature is the effect of the aforementioned sub-cavity volume. Kuhlman et al. (2007) originally 
hypothesized that the sub-cavity volume plays a crucial role in the heat transfer processes such as the onset of boiling and dry out, thus contributing to CHF. The cavity created by an impinging droplet consists of a thin liquid film, comprised of some cool initial drop liquid and some initial heated film liquid. Thus, enhanced local heat fluxes are expected in the cavity region due to the close contact of the cooler droplet liquid with the surface. It is expected that the sub-cavity volume due to higher local heat fluxes, then would be vulnerable to boiling and subsequent dry out before the cavity is refilled by a surface tension wave or covered over by subsequent nearby drop impacts. The combined effect of the localized cavity dry outs would contribute significantly to the onset of CHF.

Evidence of the influence of the sub-cavity volume is supported by observations in the literature. In spray cooling experiments, it has been commonly observed that the highest heat fluxes occur in the spray region with the highest frequency of impinging droplets (Hung et al., 2004, and Shedd and Pautsch, 2005). This shows that the impinging droplets are crucial for spray cooling. However, this correlation cannot be directly linked to the cavities. More direct evidence and the main motivation of the current work can be found in CFD simulations performed by Sarkar and Selvam (2009). Investigating the enhanced local heat flux of the vapor bubble contact line, Sarkar and Selvam (2009) found Nusselt numbers as high as 420 - 490. Their results also show that the cavity formed by a single impinging drop in the vicinity of a nucleated bubble, as depicted in their Figure 3 (c), also has a enhanced localized transient heat flux (maximum Nusselt number of 420). The percent increase of this cavity heat flux is comparable to their calculated average heat flux increase along the contact line of the nucleating bubble on the heater surface. The figure also shows that the cavity cools a significantly larger area than the vapor bubble contact line. Similar trends were observed in experiments by Horack et al. (2004) where local heat fluxes as high as nominally $50 \mathrm{~W} / \mathrm{cm}^{2}$ for a FC-72 spray were measured for the contact line with a microheater array using 
internal reflection techniques. Additionally, Soriano et al. (2010) and Gehring et al. (2010) observed that the highest temperature gradients for their experiments and simulations on single drop trains impacting a heated surface consistently occurred at the center of the droplet impact site. This impact site was where they consistently measured the thinnest film thickness. Soriano et al. (2010) used thermal imaging to acquire temperature profiles for a surface heated by an Indium Tin Oxide heater for HFE 7100, for the conditions listed in Table 1.1 that was impacted by a train of droplets. Results for both conditions showed that the lowest temperature of nominally $61^{\circ} \mathrm{C}$ occurred at the impact location. Surface temperature rose to nominally $75^{\circ} \mathrm{C}$ at the maximum spreading radius, while the impinging droplets had a temperature of $22^{\circ} \mathrm{C}$. Single water drop train experiments and simulations performed by Gehring et al. (2010) included droplet frequencies of $19.7 \mathrm{kHz}$ to $105 \mathrm{kHz}$, with droplet sizes ranging between $82.1 \mu \mathrm{m}$ and $123.5 \mu \mathrm{m}$ for average overall heat fluxes of $11.8 \mathrm{~W} / \mathrm{cm}^{2}$

to $16.4 \mathrm{~W} / \mathrm{cm}^{2}\left(T_{\text {surface }} \approx 122^{\circ} \mathrm{C}\right)$. Simulations at the impact centerline show average local wall temperatures equivalent to the droplet temperature of $17^{\circ} \mathrm{C}$. This shows direct evidence that the cool droplet liquid actually penetrates an initial liquid film to contact the heater surface (Gehring et al., 2010).

Table 1.1: Single train droplet frequency, diameter, spreading area, and maximum heat flux for experiments by Soriano et al. (2010).

\begin{tabular}{|c|c|c|c|c|}
\hline Frequency $(\mathrm{kHz})$ & $d(\mu \mathrm{m})$ & $\begin{array}{c}\text { Spreading } \\
\text { Diameter }(\mathrm{mm})\end{array}$ & $\begin{array}{c}\text { Spreading } \\
\text { Area }\left(\mathrm{mm}^{2}\right)\end{array}$ & $\begin{array}{c}\text { Max } \\
\text { Heat Flux }\left(\mathrm{W} / \mathrm{cm}^{2}\right)\end{array}$ \\
\hline 2.97 & 222 & 1.65 & 2.14 & 18.8 \\
\hline 12.0 & 318 & 2.13 & 3.56 & 22.2 \\
\hline
\end{tabular}

The primary purpose of the present work was to utilize unheated single drop experiments to quantify the sub-cavity volume history for a range of droplet impact scenarios relevant to realistic sprays. To accomplish this, a time resolved non-contact chromatic CHRocodile (CHR) thickness measuring sensor was utilized to measure the liquid film thickness beneath the cavity, referred to herein as the sub-cavity film thickness. Similar optical instrumentation has initially been utilized by 
Van Hinsberg et al. (2010) to profile the time-dependent sub-cavity liquid film thickness at the impact centerline of single impinging droplets. The current work expands upon the experimental methods developed by Van Hinsberg et al. (2010) to radially map the sub-cavity film thickness distribution in the cavity versus time. Subsequent sub-cavity volume histories are then computed through integration along the bottom cavity radius for each time step, as first documented by Hillen et al. (2012 and 2013). To the author's knowledge no other work has attempted to experimentally measure the sub-cavity film thickness versus both radius and time, or to compute sub-cavity volume histories. Figure 1.2 is an example of an impinging drop on an initial liquid film with its major features defined.

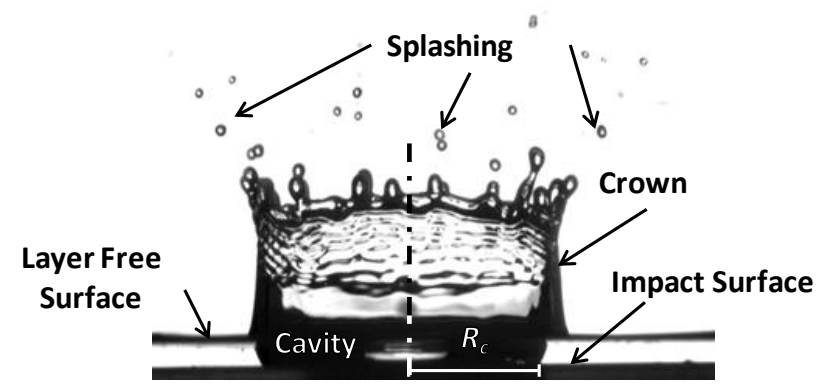

Figure 1.2: Single drop impingement into an unheated initial liquid surface and its components (Hillen et al. 2013).

In order to create a relevant test plan for the single drop sub-cavity experiments, a Phase Doppler Particle Analyzer (PDPA) was utilized to profile a full cone water spray of interest. The spray was generated by a Spraying Systems FullJet 1/8-G nozzle (see Figure 1.1) and characterized in terms of overall integrated average values of dimensionless Weber, Reynolds, and Froude numbers. This was the same nozzle considered by Kreitzer (2010), where the spray droplet size and velocity distributions used were recorded at $1.0 \mathrm{~cm}$ to $1.5 \mathrm{~cm}$ beneath the nozzle tip using FC-72 as the coolant. However, high-speed videos showed the spray not to be fully atomized within this region (Kreitzer, 2010). This fact combined with the different fluid properties justified the new measurements further downstream and using water as the liquid. PDPA was also employed herein to 
measure the droplet characteristics of the secondary droplets, referred to herein as splashed droplets, generated by the spray impacting onto a smooth surface as is shown in Figure 1.1. Though not expected to be a major contributor, the splashed droplets have been investigated to observe any contributions to cavity formations on the heater surface. This analysis of the splashed spray droplets was not the primary focus of the presented work, but has been documented in detail for future implementation into the MC model to improve the validity of mass conservation in the model.

Dimensionless numbers have been identified that allow the single droplet experimental parameters to be matched to the corresponding spray results. The most important of these is the Weber number, which is the ratio of inertial force of the impinging droplet to surface tension forces as defined by:

$$
W e=\frac{\rho v^{2} d}{\sigma}
$$

For spray cooling the Weber number has a significant effect on the heat transfer process and it has been found to be directly relatable to the CHF (Silk et al., 2008 and Kim, 2006). The Weber number also is typically used for single droplet correlations due to the dominating effect of surface tension in the impingement process. Other important dimensionless numbers include the Reynolds, Froude, and Ohnesorge numbers. The Reynolds number is the ratio of inertial force of the impinging drop and viscous forces, as defined in Equation 1.2. The Froude number shown in Equation 1.3, is the droplet inertial force to the pressure force of a gravitational surface wave. The Ohnesorge number expressed in Equation 1.4, relates the viscous force to the square root of the inertial and surface tension forces. Note that only three of these four dimensionless parameters are independent.

$$
\begin{gathered}
R e=\frac{\rho v d}{\mu} \\
F r=\frac{v^{2}}{g d}
\end{gathered}
$$

\section{Page 7}




$$
O h=\frac{\mu}{\sqrt{\rho \sigma d}}=\frac{\sqrt{W e}}{R e}
$$

Dimensionless film thicknesses have been calculated as shown in Equation 1.5. The present work has focused primarily on thin preexisting liquid films, following the terminology of Cossali et al. (1997) $\left(h_{0}{ }^{*}<1.0\right)$, or on thin and intermediate films, using the classification of Vander Wal et al. (2006) $\left(h_{0}{ }^{*} \leq 0.1\right.$ and $h_{0}{ }^{*} \approx 1$, respectively). These cover the typical ranges of spray drop size and the residual liquid film thicknesses.

$$
h^{*}=\frac{h}{d}
$$

The experiments and results in this thesis are presented in the following order: first the PDPA spray data is analyzed and used to create a detailed single droplet test matrix. Then the bottom inside cavity thickness distribution versus cavity radius and time for the ranges of We, Re, and $h_{0}{ }^{*}=h / d$ that are expected in the spray are presented.

The organization of this thesis presents a background and literature review in Chapter 2 . The experimental apparatus and procedures for the spray and single drop experiments are presented in Chapters 3 and 4 respectively. The methodology to compute the single drop sub-cavity volume is documented in Chapter 5. The analyzed spray data used to create the detailed single drop test matrix is detailed in Chapter 6. Additionally, the splashed droplet results from the spray are also included in Chapter 6. Chapter 7 contains the single drop experimental results, including a brief summary of some preliminary experiments. The main content of Chapter 7 includes the bottom inside cavity radius histories, flow visualization, bottom inside cavity thickness distribution versus radius and time, and the computed sub-cavity volume histories for the selected ranges of $W e, R e$, and $h_{0}{ }^{*}$. Conclusions and recommendations are documented in Chapter 8. Appendixes A through Appendix E 
contain additional figures and a more detailed explanation of the single drop glycerol-water mixture calculations. 


\section{CHAPTER 2: TITERATURE REVIEW}

\section{Section 2.1: Introduction}

The present literature review provides overviews of three primary topics: an existing MonteCarlo (MC) model by Kreitzer (2010), single droplet impact dynamics, and single drop cavity dynamics. This is not meant to be a comprehensive review of the MC model, or of the single drop impingement process, but is meant to provide readers with a basic background for a better understanding of the presented work. The main focus of this literature review is on the cavity created by a single droplet impinging onto an initial liquid layer, which is shown for a typical splash condition in Figure 1.2.

A general overview of Kreitzer's (2010) MC model is covered in Section 2.2, which provides a brief explanation of how the current model functions and includes the incorporated time scale arguments. The missing components needed to improve model's validity in order to develop it into a quantitative design tool are also discussed.

Section 2.3 provides a general background to familiarize the reader with the droplet impingement process and its outcomes. The main focus is on single droplets impinging onto a wetted surface, so this is inferred unless otherwise stated. Crown and splashing formation (see Figure 1.2) are discussed including a review of the various influential parameters. Also discussed are the different splash styles and the characteristics of splashed droplets. An overview of the varying droplet impingement outcome criteria and the differences for spray droplet impingements are provided.

Section 2.4 reviews previous studies of the cavity dynamics of single drop impacts. This section contains material directly relevant to the present single droplet experiments encompassing the cavity evolution and various parameters that influence it. Initial sub-cavity film thickness measurements and their trends are discussed in detail in this section. 
Section 2.5 reviews the existing work that separately defines the fluid composition of the crown, splashed droplet, and sub-cavity for a single drop impingement on a liquid film.

The origins and justification for the $h_{0}{ }^{*}$ utilized in the single drop cavity experiments in Chapter 7 is detailed in Section 2.6. This section summarizes the residual film thicknesses generated by a spray impinging upon a flat surface measured by other works.

Finally, Section 2.7 provides a brief background on how PDPA individually collects droplet diameters and velocities.

\section{Section 2.2: Current Monte-Carlo Spray Cooling Model}

The existing $\mathrm{MC}$ model uses a random number generator to assign droplet size, velocity, and impact location information based on preexisting characterization of the droplet size distribution, velocity, and flux distribution for a spray of interest. The model incorporates a series of time scales for the spray and individual droplet impacts to approximate the time evolution of the spray impingement process. The time scale concept originated from Kuhlman et al. (2007) where the spray process was broken down into individual smaller length and time scales that were computed based on dimensional analysis for each of the relevant processes. Equations 2.1 through 2.7 consist of some of the characteristic time scales computed by Kuhlman et al. (2007) for the droplet impingement, droplet cavity formation, cavity fill, and cavity dry out due to boiling. The characteristic interaction time scale between the droplet and heater surface is given by:

$$
t_{\text {inter }}=d / v
$$

The time scale for the cavity to expand to its maximum radius, or "cavity growth" time scale, is computed by:

$$
t_{c g}=12.5 t_{\text {inter }}
$$


Equation 2.2 was developed from experimental spray results by Sivakumar and Tropea (2002). Assuming the surface tension forces to be dominant the Weber number, Equation 1.1 can be set to one to solve for the cavity wave propagation by:

$$
v_{c w}=\left(\frac{\sigma}{\rho h_{0}}\right)
$$

The cavity wave travels at a higher velocity than the gravity wave, thus the time scale for surface tension to refill the cavity is estimated as:

$$
t_{f i l l}=R_{c} / v_{c w}
$$

Therefore the time scale of the total cavity lifetime is the sum of Equations 2.2 and 2.4 given by:

$$
t_{\text {total }}=t_{c g}+t_{f i l l}
$$

The time scale for a cavity to be refilled by a neighboring drop impact is computed as

$$
t_{\text {cover }}=\left(\frac{\text { Vol }_{\text {droplet }}}{Q}\right)\left(\frac{A_{\text {heater }}}{A_{c}}\right),
$$

where the droplet flux is assumed to be uniform with no overspray, and $V_{\text {ol }} l_{\text {droplet }} / Q$ is the number of spray droplets per second. The ratio $A_{\text {heater }} / A_{c}$ in Equation 2.6 is based on the droplet number flux onto the heater surface area and cavity area.

Again assuming a uniform spray flux with no overspray the time scale for the thin liquid film in a cavity to dry out can be estimated by:

$$
t_{d r y}=\frac{\left[m_{c} C_{p}\left(T-T_{s a t}\right)+m_{c} h_{f g}\right]}{q^{\prime \prime} A_{c}}
$$

A summary of the time scales used in Kreitzer's (2010) MC model are listed in Table 2.1 encompassing the individual drop impacts, the cavity lifetimes, and heater effects on the cavity. 
Table 2.1: Summary of Monte-Carlo simulation time scales for a drop with a $D_{32}=48 \mu \mathrm{m}, V=$ $12 \mathrm{~m} / \mathrm{s}, \mathrm{FC}-72$ at a flow rate of $10 \mathrm{GPH}$, and heater power of $100 \mathrm{~W}$ (Kreitzer, 2010).

\begin{tabular}{|l|c|}
\hline \multicolumn{1}{|c|}{ Time Scale } & Time $(\boldsymbol{\mu s})$ \\
\hline Time between droplet impacts & $5.05 \mathrm{E}-03$ \\
\hline Time of droplet impacts & 0.8 \\
\hline Time for fastest cavity to move 1 pixel & 2.2 \\
\hline Time between impacts in a cavity & 4.5 \\
\hline Time of cavity formation & 32 \\
\hline Time for cavity to reach boiling & 75 \\
\hline Time to cause droplet to boil and vaporize & 121 \\
\hline Time of crown collapse & 160 \\
\hline Time to heat droplet 30 degrees and vaporize & 397 \\
\hline Time of surface tension wave to fill in cavity & 60 \\
\hline Time of gravity wave to fill in cavity & 1224 \\
\hline
\end{tabular}

The model assumes droplets above the deposition/rebound criteria (which will be covered below) that impact the liquid film create instantaneous fully formed axisymmetric cavities. A presumed maximum cavity radius of 2.7 times the droplet diameter is used at a dimensionless time value of $\tau=5$ computed by:

$$
\tau=t(v / d)
$$

These individual cavities are tracked as they move across the heater and throughout their lifetime. A minimum sub-cavity film thickness of $1 \%$ of the droplet diameter was assumed based on CFD simulations performed by Cole at al. (2005). The cavities retain their full size throughout their lifetimes that are governed by the surface tension refill time scales unless the cavity is covered over by subsequent drop impacts. This timescale was chosen since it was shorter than that for the gravity wave, as listed in Table 2.1. To account for neighboring impacting droplets, if a particular cavity had more than $50 \%$ of its area overlapped by a newer cavity, or cavities, then the preexisting cavity was considered to have been filled. This logic was incorporated into the model instead of the using the time scale in Equation 2.6. The cavities themselves were color coded for visualization purposes to represent the state of the sub-cavity film fluid at any time in the process: dark blue for undisturbed liquid film, medium blue for cavities that did not form a thin film at the heater surface, light blue for 
cavities that fully penetrated the initial liquid layer that are below the onset of boiling, orange for the onset of boiling in a cavity, and red for when the cavity experienced dry out prior to being recovered by nearby drop impacts, or filling in due to surface tension (Kreitzer, 2010). This is shown for the initial Monte-Carlo screen shot in Figure 2.1.
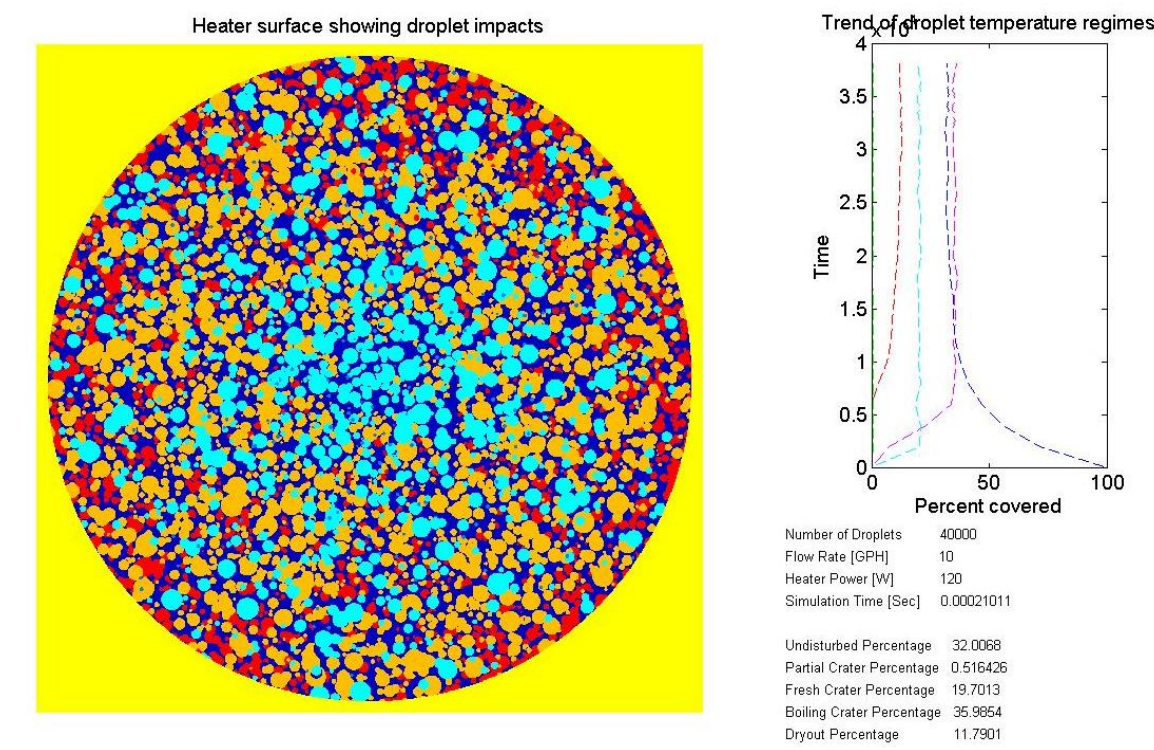

Figure 2.1: Simulated heater surface from the MC Spray Cooling model (Kreitzer, 2010).

The model was qualitatively acceptable and demonstrated realistic trends for surface dry out. Results in Kreitzer (2010) showed that the first portions of the heater that experienced dry out occurred on the outer edges of the heater which is consistent behavior with spray experiments in Pautsch and Shedd (2006) where they only observed film boiling at the outer region of the spray. However, even though the model has shown promise, it still needs to incorporate mass conservation of the film flow and fluid ejected by secondary droplets, improved cavity formation time scales, and improved values of the sub-cavity volumes and lifetimes. The present work focuses on experimental determination of the cavity time scales and sub-cavity volumes to be incorporated into the MC model to improve cavity dry out time prediction. 


\section{Section 2.3: Single Drop Impingement}

Impinging droplets can come in many shapes including spherical, deformed, ellipsoidal, oscillate, and the droplets can have internal circulation (Rein, 1993, and Yarin, 2006). Impingements may be normal to the wall, at oblique angles, or on moving surfaces (Rein, 1993). Impact angles are defined using the commonly accepted definition, shown schematically in Figure 2.2, with the angle measured from the normal to the surface. Both reviews by Rein (1993) and Yarin (2006) explain that the impingement process for any droplet is dependent on the droplet size, impact velocity, impact angle, fluid properties, surface type, surface roughness, and (for a dry surface) surface wettability. There are two main basic droplet impingement processes: impacts on dry surfaces and impacts on wetted surfaces. These different surface conditions result in some similar outcomes, or in radically different outcomes. Rein (1993) mentions that the splashing characteristics of droplet on a dry surface are similar to those on a thin liquid film, while work done by Rioboo et al. (2000) show that they have different morphologies, spreading behaviors, and splashed droplet formation.

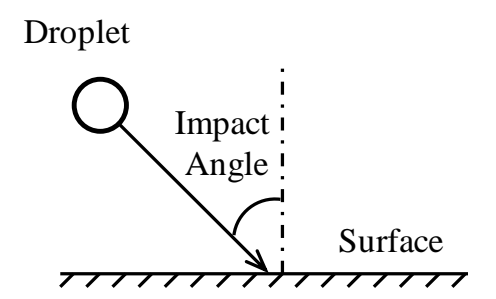

\section{Figure 2.2: Droplet impact angle.}

When a droplet impacts upon a dry surface it can either spread, bounce, splash, cavitate (rare cases), or hover at Leidenfrost temperatures (Rein, 1993, and Yarin, 2006). The determination of splashing for drop impact on a dry surface depends upon the drop diameter to surface roughness ratio, and the kinematic and fluid properties of the drop (Mundo et al., 1995).

Outcomes of a droplet impingement onto a liquid surface include rebound, deposition, partial rebound/deposition, floating, and the generation of a splash (Rein, 1993). Impinging drops of 
sufficiently large $W e$ and $R e$ generate a propagating corona/crown where splashed droplets can be shed from crown rim breakup (Yarin, 2006).

The single drop experiments in the present work focus on drop impingement into a static thin liquid layer normal to the surface. Thus, dry surface behavior and the effect of the solid surface geometry are unimportant and are not expanded upon further. The spray investigated in the current work consists of droplets impinging normal to the wall or at oblique angles. Additionally, the effects of oscillations of the drops in the single droplet experiments are neglected since Rein (1993) discusses that it is widely believed that any oscillations are dampened upon impact.

\section{Section 2.3.1: Crown Morphology and Formation}

A general description of the crown evolution due to an impinging drop on a wetted surface was found in Yarin (2006). During the initial impingement process, inertia and surface tension forces dominate as liquid is displaced from the impact location forming the crown. An undisturbed crown will continuously propagate radially and in height until surface tension and gravity forces cause it to no longer grow, and then collapse. A collapsing crown is followed by a surge of fluid rushing to refill the cavity that, for $h_{0}{ }^{*}>1.0$, can result in the subsequent formation of a central spike of liquid commonly known as the Worthington jet (Yarin, 2006). Splashing originating from the crown is believed to be a result of perturbations in the crown rim that cause cusps to form. Subsequently, jets are formed from the cusps in which splashed droplets pinch off due to capillary instabilities (Yarin, 2006). Cossali et al. (2004) observed in their single drop experiments that splashing crowns typically result in at least 10 jets from which splashed droplets may detach. An example of a splashing drop impingement can be seen in Figure 1.2.

Macklin and Metaxes (1976) observed in their experiments that the maximum crown height was $0.5-4.0$ times the initial droplet diameter. The crown radius size is much larger than the initial 
droplet diameter (Mundo et al., 1995) and the generally accepted crown radial growth rate derived from single droplet experiments is dependent on the square root of time (Yarin, 2006). Sivakumar and Tropea (2002) measured crown radii formed by a spray that were nominally 2.5 times the initial droplet diameter. Cossali et al. (2004) reported slightly different crown growth rates of $t^{0.43}$ in their experiments. They also found that the Weber number has a strong influence on crown height, but not the crown formation or speed of collapse.

The main contributing parameters to the crown morphology of a drop impingement besides impact velocity are the fluid properties and initial liquid film thickness. Experiments performed by Vander Wal et al. (2006) varied the viscosity of working fluid and found that the crown surface became smoother at higher Weber numbers. More influential on the crown shape is the dependence of the crown angle (the angle between the crown side and the free surface) on $h_{0}{ }^{*}$ (Fedorchenko and Wang, 2004). For drop impingements on a liquid film thickness of $h_{0}{ }^{*}<0.1$, Chen and Wang (2000) showed that the upper portion of the crown was much larger in diameter than the bottom portion resulting in a "bowl" shape. Fedorchenko and Wang (2004) investigated this effect further and reported that $h_{0}{ }^{*}<0.25$ resulted in acute crown angles while larger $h_{0}{ }^{*}$ resulted in crown angles of at least $90^{\circ}$. Variations in surface temperatures below the Leidenfrost temperature are expected to have an indirect effect, in the form of influencing the fluid properties, on the film properties according to Cossali et al. (1997). Therefore, single drop experiments may be extrapolated to hot wall conditions.

Even though the present results focus on the sub-cavity film dynamics, the crown morphology and evolution are important to gain a basic understanding of the impingement process. The cavity size and lifetime are directly influenced by the formation and evolution of the crown. Single drop impingements results, discussed in Chapter 7, were observed to follow many of the behavior characteristics described in this section. 


\section{Section 2.3.2: Splashing}

The splashing phenomenon can be subdivided into four groups: prompt, delayed, film, and Worthington jet splashing (Cossali et al., 1997, Manzello and Yang, 2002, Vander Wal et al., 2006, and Kalantari and Tropea, 2007). The prompt and delayed splash consists of secondary drops that are ejected from the crown during expansion and collapse respectively, as defined by Cossali et al. (1997) and Vander Wal et al. (2006). Film splashing occurs when liquid ligaments are ejected directly from the film (Manzello and Yang, 2002, and Kalantari and Tropea, 2007). The Worthington jet splashing occurs when secondary drops detach from the central Worthington jet (Cossali et al., 1997, Vander Wal et al., 2006).

There are many contributing variables that influence the splashing criteria. Cossali et al. (2004) summarized that the splashing criteria are influenced by the impinging droplet characteristics, surface temperature, surface roughness and wettability (for dry surfaces and extremely thin initial films), and the initial liquid film thickness. Experiments by Shin and McMahon (1990) focusing on Worthington jet formations show that thicker $h_{0}{ }^{*}$ actually promote Worthington jet splashing up to a certain point. They found that for thinner target liquid layers the wall greatly influences the outcome of the Worthington jet and could dampen it when the wall was replaced with a sponge. Cossali et al. (1997) showed that crown splashing is dependent on viscosity and a thicker $h_{0}{ }^{*}$ actually can inhibit crown splashing. Xu et al. (2005) reported that variation in the surrounding gas pressure for a single drop impacting onto a smooth dry surface had a pronounced influence on the onset of splashing. Vander Wal et al. (2006) concluded that high surface tension and viscous forces inhibit splashing (i.e. large We and Re promote splashing), consistent with Cossali et al. (1997).

Several authors have attempted to identify criteria to predict the outcome of an impinging droplet. Initial development of splash criteria incorporating the surface tension and viscosity was 
developed by Mundo et al. (1995) for droplets impacting onto a solid surface. The following is the most prominent correlation that accounts for the viscous and surface tension effects (Yarin, 2006):

$$
K=W e O h^{-0.4}
$$

Splashing typically occurs for the criteria $K>57.7$ for Equation 2.9. Cossali et al. (1997) expanded Equation 2.9 further by incorporating the initial film thickness in the correlation:

$$
Y=K /\left[2100+5880\left(h_{0}^{*}\right)^{1.44}\right]
$$

where splashing consistently occurred for criteria limits of $Y>1$. Wang and Chen (2000) reported that the critical splashing Weber number was insensitive to variations of $h_{0}{ }^{*}<0.1$, but was dependent on viscosity and surface roughness. Contrary to this, Riobo et al. (2003) did observe a correlation of $h_{0}{ }^{*}$ with the splash criteria while varying $0.06<h_{0}{ }^{*}<0.15$. Table 2.2 is a summary of some of the criteria and critical limits of droplet impingement outcomes reported in the literature for the rebound, deposition, crown formation, and splashing.

Table 2.2: Summary of various deposition/rebound/splash criteria reported in literature.

\begin{tabular}{|l|c|c|c|}
\hline \multicolumn{1}{|c|}{ Author } & Wet/Dry & Criteria & Critical Criteria Limit \\
\hline Ching et al. (1984) & Wet & Rebound/Deposition & $W e=20$ \\
\hline Lee and Hanratty (1988) & Wet & Rebound/Deposition & $W e=20$ \\
\hline Wang and Watkins (1993) & Spray & Rebound & $W e<30$ \\
\hline Rein (1993) & Wet & Rebound/Deposition & $W e<3.0$ \\
\hline Rein (1993) & Wet & Splash & $W e=7.9 \mathrm{e}^{10} \mathrm{Oh}^{2.8} \approx 60$ \\
\hline Mundo et al. (1995) & Dry & Splash & $K=57.7$ \\
\hline Cossali et al. (1997) & Wet & Deposition/Splash & $Y=1 ; 0.1<h_{0}{ }^{*}<1.0$ \\
\hline Rioboo et al. (2003) & Wet & Deposition/Crown & $K=440 ; h_{0}{ }^{*}<0.06$ \\
\hline Rioboo et al. (2003) & Wet & Crown/Splash & $K=2100 ; 0.06<h_{0}{ }^{*}<0.15$ \\
\hline Vander Wal et al. (2006) & Wet & Deposition/Splash & $W e^{0.5}>20$ \\
\hline Kalantari and Tropea (2006) & Spray & Deposition & $W e<2$ \\
\hline Kalantari and Tropea (2006) & Spray & Partial Deposition & $30<W e<80$ \\
\hline Kalantari and Tropea (2006) & Spray & Splash & $W e>80$ \\
\hline
\end{tabular}

The criteria limits listed in Table 2.2 are not hard limits, but predict the trends reasonably well. These criteria helped determine in the present work the droplet conditions that would produce a 
cavity to examine for the single drop experiments. Also, a basic comprehension of the splashing process has aided with the analysis of the measured splashed spray droplets discussed in Section 6.3.

\section{Section 2.3.3: Splashed Droplet Characteristics}

Macklin and Metaxes (1976) observed that the volume of the splashed droplets created from droplets impinging onto shallow $h_{0}{ }^{*}$ was two to three times that of the impacting droplet. Mundo $e t$ al. (1995) utilized Phase Doppler Anemometry (PDA) to characterize the splashed droplets created by a train of single droplets. They measured the splashed droplets to be (0.12 to 0.80$)$ and (0.08 to 0.28) times the respective $D$ and $D_{32}$. According to Cossali et al. (1997), the splashed droplet size and velocity are dependent on viscosity, and later work by Cossali et al. (2004) found film thickness did not influence splashed droplet characteristics. Worthington jet splashed droplet sizes have been observed by Fedorchenko and Wang (2004) to be of the same order of magnitude as the initial droplet. Cole et al. (2005) developed a Lagrangian spray wall impingement model that was integrated into the Eulerian free surface model (Volume of Fluid). It enabled the modeling of spray impingement and splashing, including fluid film effects on the wall. Their results showed that up to 15 - 18 times the droplet volume was splashed in their single drop simulations. The highest total splashed volumes were for the Worthington jet. Kalantari and Tropea (2006) separated the splashed droplets from impinging spray droplets based on the axial velocity sign convention in their PDA data. They found that the splashed droplets were 0.8 to 1.2 times the size of the spray droplets and their axial velocities never exceeded $3 \mathrm{~m} / \mathrm{s}$. They explained that interaction between neighboring impinging droplets could feed excess fluid into the crown, thickening it, which will produce larger splashed droplets. In addition, Kalantari and Tropea (2006) mentioned that ligaments ejected from the film wall which may become spherical over time could also contribute to the larger splashed droplet sizes. Splashed droplet sizes similar to the initial droplet were reported by Okawa et al. 
(2007) for a train of droplets impacting at oblique angles. They recorded splashed droplet masses of upwards of 100 times that of the initial droplet when increasing the impact angle (defined in Figure 2.2), but recorded splashed droplets $1 / 10$ the size of the initial drop at small impact angles.

In summary, crown splashed droplets for single impinging droplets are numerous and small in size compared to the initial droplet. However, in spray conditions where there are many interacting droplet impacting at varying angles the splashing outcomes becomes dramatically more complicated. The method used by Kalantari and Tropea (2006) to extract the splashed droplet characteristics from the spray did not take into account any of the splashed droplets returning to the impingement surface; an improved technique that does this is covered in Section 3.5.

\section{Section 2.3.4: Spray Impingement Versus Single Droplet Impingement}

Behavior of droplet impingements in a spray is much more complex than single droplet conditions. Sivakumar and Tropea (2002) found in their spray experiments the crowns grew at $t^{0.2}$ instead of $t^{0.5}$, as seen for single droplet impacts, while observing irregularities in the crown and splashing formations. They explain the irregularities and slower crown evolution times are due to nonuniformity in the spray residual film, and the interaction between impinging droplets. Results in Kalantari and Tropea (2006) also showed slow crown growth $\left(-0.2 t^{0.3}\right)$ and receding in a spray consistent with Sivakumar and Tropea (2002).

Even though both mentioned authors in this section argue against extrapolating single drop results for a full spray, single drop experiments are vital to understanding the underlying physics occurring in a spray process. The present author was unaware of any literature investigating the effects of real spray condition influences on the sub-cavity film thickness and thus the sub-cavity volumes. 


\section{Section 2.4: Cavity Dynamics}

Some of the initial detailed studies of the cavity created by an impinging drop were performed by Engel (1965 and 1967). Engel (1965 and 1967) experimentally observed the cavity formation for droplets impinging into a deep pool and reported that the cavities formed hemispherical shapes. Through energy balances, Engel analytically developed a model to predict the cavity depth versus time. She assumed that half of the impact energy of the droplet was transferred into the target liquid, and her predictions were in good agreement with experimental data. Experimental observations for single drops impinging onto varying liquid film thicknesses by Macklin and Hobbs (1969) showed that lowering initial liquid film thickness resulted in a flattening of the hemispherical cavity into a cylindrical shape. Building off of Engel (1967) Macklin and Metaxas (1976) developed an analytical model that predicted the inside cavity radius and crown height for drop impingements on shallow surfaces. They also experimentally observed cylindrical cavity shapes for shallow pool impacts and defined deep pools to be when the wall had no influence on the cavity evolution. The cylindrical shape of the cavity for shallow pools was again observed by Shin and McMahon (1990), and they state that a cavity will have a deep pool behavior for target liquid depths more than twice the hemispherical cavity radius.

Detailed analysis of cavity evolution for $0.5<h_{0}{ }^{*}<2.0$ were performed by Roisman et al. (2008). Roisman et al. (2008) reported that the viscous effects on the cavity formation are negligible at high Reynolds and Weber numbers. They experimentally measured the cavity radius, which compared well with their theoretical model, and found that the cavity radius initial growth rate follows the same general $t^{0.5}$ growth rate of the crown. Roisman et al. (2008) explained that though inertia governs the drop impingement, surface tension and gravity forces govern the cavity growth at later stages which are responsible for its deviation from $t^{0.5}$. In their experiments they noticed a wave propagating downward from the top of the inside cavity surface that they indentify as a capillary 
wave. This wave was observed earlier by Fedorchenko and Wang (2004). Roisman et al. (2008) further report that the Reynolds number dominates the sub-cavity film flow. Berberovic et al. (2009) performed, CFD simulations, analytical analysis, and experiments for three liquids focusing on single drop cavities for $0.5<h_{0}{ }^{*}<2.0$. They provide a detailed description of the cavity evolution and report that the initial cavity diameter follows the $t^{0.5}$ growth rate while gravity and surface tension forces govern the later times, consistent with Roisman et al. (2008). Section 7.5 gives a detailed description of the cavity evolution. Berberovic et al. (2009) also explains that the Weber number dominates the maximum cavity expansion and higher $W e$, and $h_{0}{ }^{*}$ increases the cavity lifetime until capillary forces cause the cavity to recede. They also observed a cavity capillary wave for nearly all of their cavity formations.

In summary, cavity formation is dramatically different for thin initial liquid films and deep pools as the wall affects cavity morphology and evolution. Many of the observations by Roisman $e t$ al. (2008) and Berberovic et al. (2009) apply to the single drop results that are presented in Chapter 7, though the origin of the cavity wave described in literature as a "capillary wave" will also be discussed in this chapter. Deep pool features are not of interest for the current work as the single droplet experiments have been conducted for thin films, so many of the behaviors recorded in literature for thin film cavities influence the sub-cavity behavior.

\section{Section 2.4.1: Sub-cavity Film Thickness}

At various points in the literature, attempts have been made to quantify the sub-cavity film thickness. Rioboo et al. (2003) stated that even though they could not measure it, they believed the sub-cavity film thickness to decrease with the initial film thickness. In a computational study by Nikolopoulos et al. (2007) the minimum film thickness was found to be $1.98 \%$ to $2.06 \%$ of the initial droplet diameter for the respective CFD mesh grids of $d / 40$ and $d / 80$ for $W e=125,250$, and 
598 at $h_{0}{ }^{*}=0.116$. Berberovic et al. (2009) concluded, based on their CFD results, that the We and $h_{0}{ }^{*}$ did not affect the centerline minimum film thickness.

Van Hinsberg et al. (2010) utilized a non-contact chromatic optical sensor to measure the impact centerline sub-cavity film thickness for single droplet impacts for three fluids, varying dimensionless parameters of $0.07<h_{0}{ }^{*}<2.0$ and $93<W e<574$. Their results showed that as a droplet impinges onto a liquid layer of finite thickness it quickly creates a virtually constant thin subcavity film thickness until the subsequent receding of the cavity. This is called the retraction phase. They also found the centerline sub-cavity film thickness to be dependent upon the initial dimensionless liquid film thickness and the impinging droplet Reynolds number, but this thickness reached a constant value at high droplet Weber and Froude numbers. Additionally, their results show the increased cavity lifetime for increasing $W e$ and $h_{0}{ }^{*}$, consistent with Berberovic et al. (2009). Hillen et al. (2012 and 2013) used a similar optical instrument to measure the sub-cavity film thickness for similar ranges of We and $h_{0}{ }^{*}$, but obtained thickness data traversing radially outward from the drop impact centerline. These data were then used to compute sub-cavity volumes versus time.

The main focus of this thesis is the sub-cavity film thicknesses, used to compute the subcavity volumes. Results from Nikolopoulos et al. (2007) are felt to be questionable since at a maximum they had only two computational cells in the minimum sub-cavity film thickness region. In addition they observed dry patches in their cavities during simulations which were not observed in the experiments and simulations with higher sub-cavity adaptive mesh grid resolutions of drops under the same impingement conditions in Hillen et al. (2012). Initial techniques developed by Van Hinsberg et al. (2010) guided the experimental methods presented the subsequent Chapter 4. Chapters 5, 6, and 7 will expand upon the presentations given in Hillen et al. (2012 and 2013). 


\section{Section 2.5: Composition of Drop and Initial Layer Fluid}

Numerous studies have been performed investigating the composition of the droplet and initial target film fluid in the crown. Rein (1993) states that the crown primarily contains the initial liquid layer fluid compared to the drop fluid. This was investigated further through numerical simulations where the drop fluid and initial layer fluid could be distinguished separately by Josserand and Zaleski (2003) and Nikolopoulos et al. (2005). They also found that the mass in the crown consists of more initial film fluid than droplet fluid, but did not give quantitative percentages. Kalantari and Tropea (2007) analytically estimated that $48 \%$ of the liquid inside the cavity is fed into the crown for $35<W e<163$ and $280<\operatorname{Re}<655$ at $h_{0}{ }^{*}=1.0$. Single drop simulations performed by Nikolopoulos et al. (2005) showed that the splashed droplets contained $73 \%$ to $88 \%$ of the mass of the initial target film fluid.

Santini et al. (2011) were able to visually show that the droplet liquid and target liquid interface are clearly distinguishable in the cavity for their deep pool single drop experiments by taking advantage of the temperature dependency of the refractive index of the fluids. CFD simulations performed by Dinc and Gray, presented in Hillen et al. (2012), were able to expand upon the techniques in Josserand and Zaleski (2003) and Nikolopoulos et al. (2005) to quantify the ratio of the initial drop liquid to target film liquid in the drop impact cavity. For the two cases of $W e$ and $h_{0}{ }^{*}$ studied it was found that the sub-cavity for $W e=633$ and $h_{0}{ }^{*}=0.11$ consisted of almost exclusively of the droplet liquid. The sub-cavity for $W e=141$ and $h_{0}{ }^{*}=0.61$ contained a greater fraction of initial film fluid though it was still predominantly drop fluid.

Though not the focus of the present research, the sub-cavity drop-target film liquid ratio is important to gaining a greater understanding of the spray cooling heat transfer mechanisms and improving the MC model. The amount of cool drop liquid present in the sub-cavity volume will have 
a direct influence on the localized transient heat flux to the sub-cavity liquid for drop impingements on a heater, and also influence the time needed to induce cavity film boiling.

\section{Section 2.6: Residual Spray Film Thickness}

Table 2.3 summarizes the droplet sizes and residual film thicknesses created by sprays from previous work. The spray film thicknesses in Table 2.3 were measured utilizing various techniques including visual measurements, optical techniques based on total internal reflection, or microtraversing a needle. The reported spray droplet diameters are arithmetic means unless otherwise noted.

Table 2.3: Summary of measured liquid film thickness created by an impinging spray and spray droplet sizes studied in literature.

\begin{tabular}{|l|c|c|c|c|c|}
\hline \multicolumn{1}{|c|}{ Source } & $\boldsymbol{D}(\boldsymbol{\mu m})$ & $\boldsymbol{W e}$ & $\boldsymbol{R e}$ & $\boldsymbol{h}_{\boldsymbol{s}}(\boldsymbol{\mu m})$ & $\boldsymbol{h}_{\boldsymbol{s}}{ }^{*}$ \\
\hline Kalantari and Tropea (2006) & $21-47$ & $2-167$ & $10-560$ & $8-107$ & $0.17-5.1$ \\
\hline Kalantari and Tropea (2007) & $25-30$ & $35-163$ & $280-655$ & $13-80$ & $0.52-3.2$ \\
\hline Pautsch et al. (2004) & $45-75$ & $147-246$ & $296-493$ & $42-118$ & $0.56-2.6$ \\
\hline Pautsch and Shedd (2006) & $45-75^{*}$ & $746-1,240$ & $1,330-2,210$ & $18-75$ & $0.24-1.7$ \\
\hline Mathews et al. (2003) & $110-350^{\dagger}$ & $1,290-1,700$ & $3,120-4,840$ & $20-50$ & $0.06-0.45$ \\
\hline Sivakumar and Tropea (2002) & $123-310$ & $414-1,096$ & $2,020-5,950$ & $54-193$ & $0.17-1.6$ \\
\hline Tilton (1989) & $118-136$ & $141-660$ & $1,180-2,370$ & $120-350$ & $0.88-3.0$ \\
\hline
\end{tabular}

* Since a spray droplet size was not specified it was assumed Pautsch and Shedd (2006) utilized the same or similar nozzle as in Pautsch et al. (2004) since the former was a continuation of the later. The Sauter mean diameter of the spray droplets was used.

\section{Section 2.7: PDPA Background}

PDPA was utilized to characterize the present spray experiments due to various advantages.

These include the ability to non-intrusively measure simultaneous individual droplet diameters and velocities with directional sensitivity; a lack of required velocity calibration; high spatial and temporal resolution; and high accuracy (Albrecht, 2003, and TSI Manual, 2005). The system uses two separate techniques, Laser Doppler Velocimetry (LDV) and Phase Doppler Interferometry (PDI) to measure the respective droplet velocities and diameters. LDV is based on a laser that generates a coherent beam in which droplets pass through, reflecting scattered light back to the receiving optics. 
Each measuring component consists of two equal intensity beams crossed at a half angle at the focal length of the lens creating the measuring volume at the intersection as shown in Figure 2.3. When droplets pass through the measuring volume light is scattered from the drops, with a light intensity that varies as the drops pass through the fringe patterns that are created. Photomultiplier tubes (PMT) produce an electrical current proportional to the scattered light variation in the fringe pattern to frequencies that can be converted into the particle velocity by Equation 2.11 (TSI Manual, 2005).

$$
v=f \frac{\lambda}{2 \sin \alpha}
$$

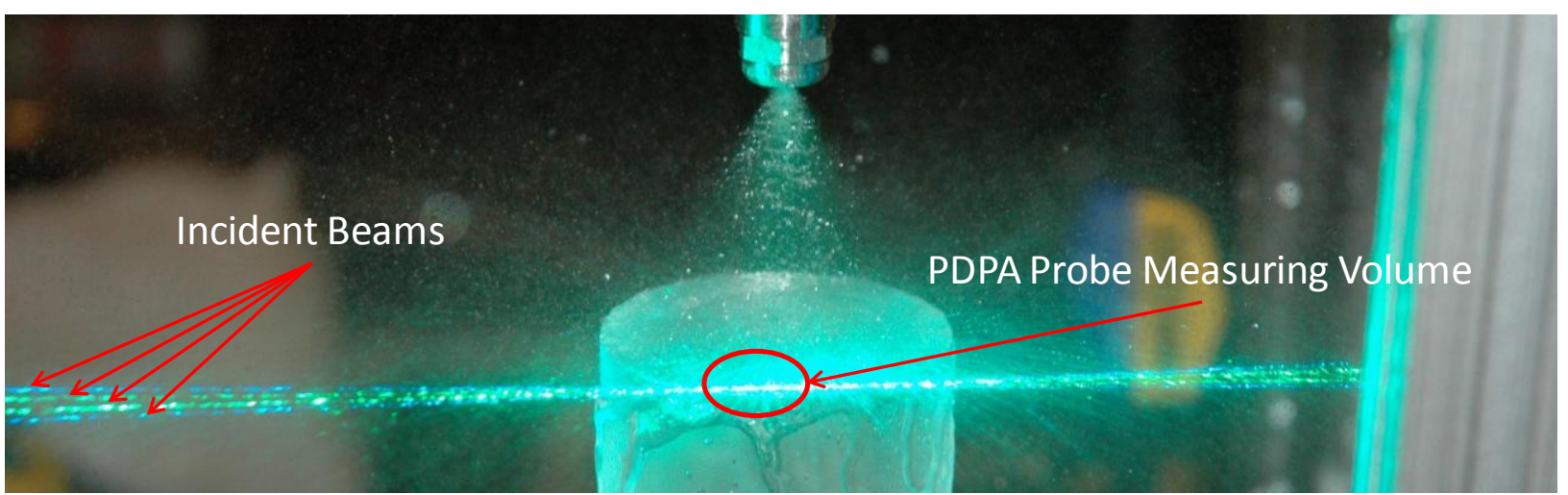

Figure 2.3: Incident beams crossing creating the measuring volume.

PDI also requires the measuring volume created by the incident laser beams to scatter light off the particles passing through. Glare points in the particle are responsible for the scattered light which affects the amplitude and phase signal of the laser measuring volume. At least two photo detectors positioned off-axis at different elevations are required to interpret the phase shift and amplitude difference of the particle traveling though the measuring volume. Thus, the diameter is inferred by the phase and amplitude differences of the signals from the moving particle (Albrecht, 2003). The diameters are computed from a phase slope given in dimensionless form in Equation 2.12 (TSI Manual, 2005).

$$
\frac{\text { Phase }}{360}=\text { "Slope" } \times \frac{\text { Detector Separation }}{\text { Receiver Focal Length }} \times \frac{\text { Particle Diameter }}{\text { Fringe Spacing }}
$$




\section{Section 2.8: Summary}

The MC model developed by Kreitzer (2010) shows promise by incorporating various micro timescales for the individual droplet impingements to simulate a spray, but is still lacking many physics that will be required in order to make the model quantitatively accurate. One parameter that could improve the MC model that is the focus of the current work is determination of the amount of sub-cavity liquid volume of the impinging droplets as a function of time. It is hoped that the subcavity volumes and respective lifetimes can help predict the onset of boiling in the cavity and thus the CHF.

A broad background of the single drop impingement process and outcomes leads to a better understanding of the cavity dynamics. The drop impingement, crown, and cavity formation process are all interconnected and dependent on each other. Cavity formation and its duration are directly dependent on the impinging droplet dimensionless parameters ( We, Re, and $F r$ ). Splashing behavior is relevant for the mass conservation of in the MC model, but in this work it provides comprehension of the measured splashed droplets in Section 6.3.

Previous work focusing on the cavity dynamics has been used in the development of the current experimental method. In addition, this information has provided insight into the single drop sub-cavity film thickness results presented in Chapter 7. The ratio of the drop liquid and initial target film thickness was not studied in depth in the present work, but is expected to be more prominent in future work.

The dimensionless residual spray film thicknesses shown in Table 2.3 have been utilized in the single drop test matrix listed in Section 6.5. These data have provided a realistic range of $h_{0}{ }^{*}$ for the single drop cavity experiments that are relevant to spray conditions. 


\section{CHAPTER 3: $\quad$ SPRAY EXPERIMENTS APPARATUS AND Procedure}

\section{Section 3.1: Introduction}

This chapter documents the apparatus and methodology of the spray experiments. The main purpose of the spray experiments was to generate a detailed test matrix for the single drop sub-cavity film thickness experiments for a proper range of relevant spray conditions. Detailed descriptions of the spray apparatus and the PDPA setup used for characterization are given in Sections 3.2 and 3.3 respectively. The methodology and procedures for measuring the spray droplet sizes and velocities via PDPA without an impact surface are detailed in Section 3.4. Initial documentation of the spray apparatus and PDPA spray characterization procedure can be found in Hillen et al. (2013).

Additionally, this chapter discusses the methodology and procedures for using PDPA to characterize the splashed droplets generated by the spray impinging onto a surface. As discussed on Chapter 1 these data sets are examined for their contributions to crater formation, but are primarily documented herein for future use. Descriptions of the spray impact surface are provided in Section 3.2.1, while the procedure for collecting and extracting the splashed drop data is presented in Section 3.5. Two main sets of splashed droplet data were collected, the first being in the spray region above the impact surface, and the second being outside of the spray region to the side of the impact surface.

\section{Section 3.2: Spray Apparatus}

Figure 3.1 depicts the spray apparatus is depicted schematically. The apparatus consisted of a recirculating loop system driven by a positive displacement pump (Tuthill model number DGS1.3PPPVNN37000). Most of the liquid was recirculated except for the fluid that was splashed when a $5.08 \mathrm{~cm}$ diameter impact surface was installed. The working fluid was distilled water, and the spray was contained within an optically transparent $76 \times 76 \times 23 \mathrm{~cm}$ Plexiglas tank open to the atmosphere. Spray flow conditions (nozzle pressure and flow rate) were measured upstream via a 
pressure gage and flow meter, and foreign particles were removed from the water by a 0.5 micron filter. The selected spray nozzle was a full cone Spraying Systems 1/8-G FullJet nozzle with $60^{\circ}$ cone angle, which produced the spray profile incorporated by Kreitzer (2010) in his original MC model. The nozzle was originally chosen for the MC model since it was determined by Yerkes et al. (2006) to give the most uniform spray flux out of several nozzles tested for spray cooling applications.

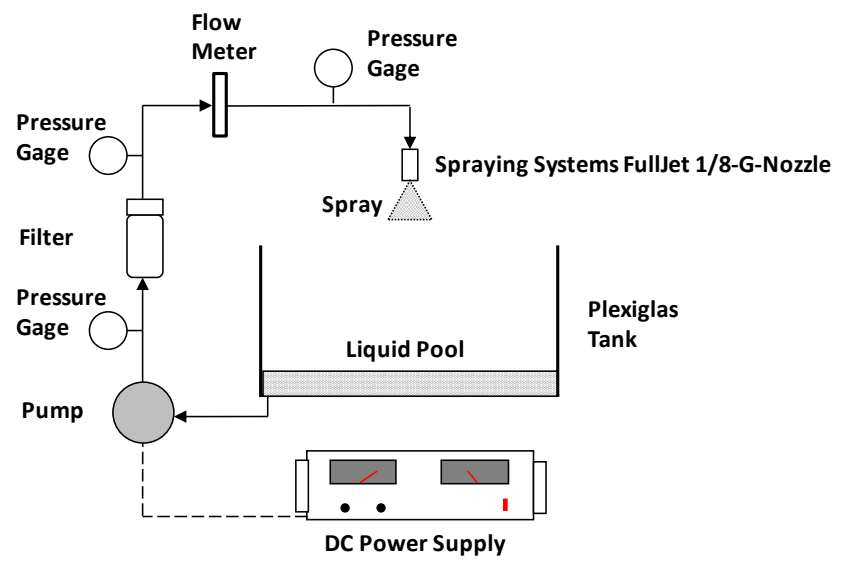

Figure 3.1: Schematic of spray apparatus (Hillen et al., 2013).

\section{Section 3.2.1: Spray Impact Surface for Splash Droplet Experiments}

Splashing droplet sizes and velocities generated by the spray impinging onto a smooth surface were also measured via PDPA for the same nozzle. The spray impact surface was a smooth $5.08 \mathrm{~cm}$ diameter and $5.08 \mathrm{~cm}$ long cylindrical acrylic pedestal that was mounted beneath the nozzle shown in Figure 3.2. The leveled impact surface was centered with the nozzle orifice exit and positioned at standoff distances from the nozzle exit such that it would be fully covered by the full spray cone with a slight overspray. 


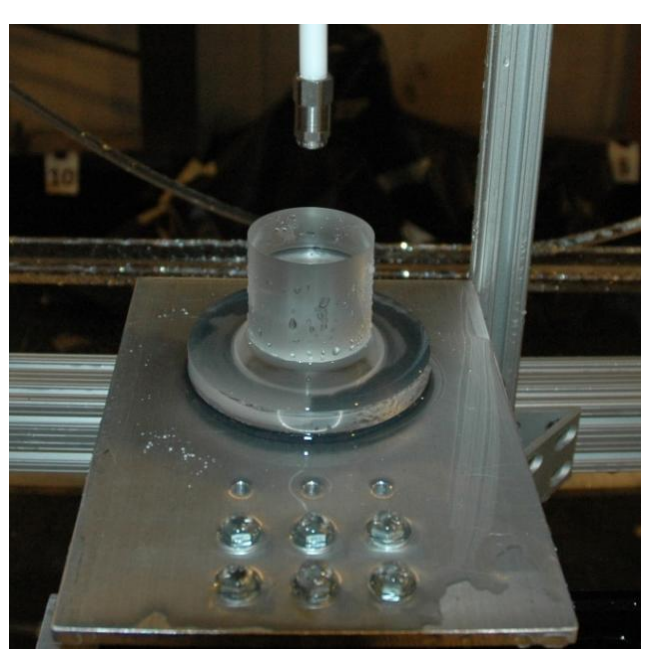

Figure 3.2: Spray testing $\mathbf{5 . 0 8} \mathrm{cm}$ diameter impact surface apparatus with nozzle.

\section{Section 3.3: PDPA Setup}

The PDPA system utilized for spray characterization was a two-component system manufactured by TSI, Inc. This system had a manufacturer reported maximum instrumentation uncertainty of nominally $0.75 \%$. The light source was a water cooled argon-ion laser (maximum 5W) that was split into four beams by a multicolor beam generator creating the two velocity measuring components. A photo detector module (PDM) contained the PMT and a $175 \mathrm{MHz}$ signal analyzer to interpret droplet size and velocity. The optics consisted of a transmitter and optical receiver that were mounted on a tri-axis traverse with a reported $10 \mu \mathrm{m}$ resolution as shown in Figure 3.3 a). The receiving optics were positioned $30^{\circ}$ off-axis from the transmitter in a backscatter optical configuration (Figure 3.3 b). Light between the optics, beam splitter, and PDM was transmitted via fiber optic cables. Control of the PDPA system and data acquisition was performed with the manufacturer's propriety software FlowSizer v2.0.4. 


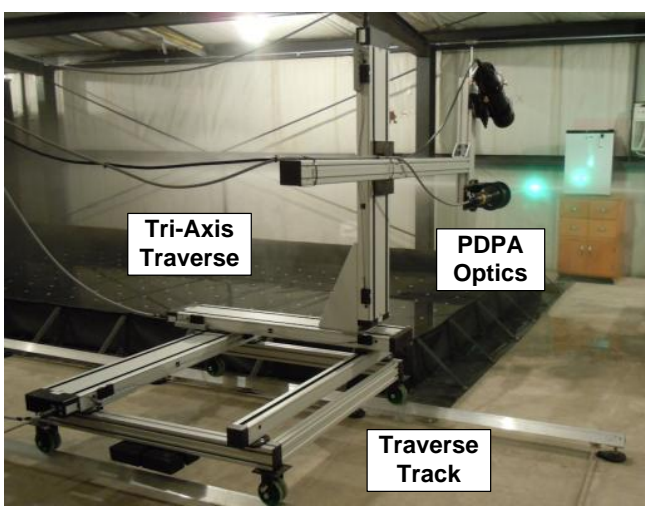

a)

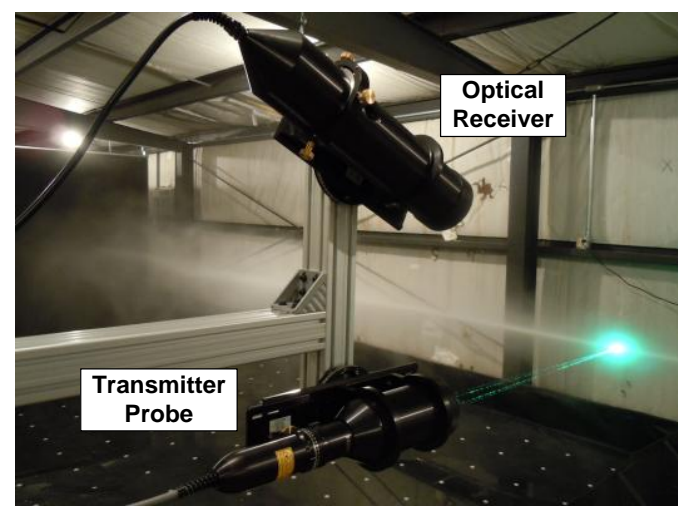

b)

Figure 3.3: PDPA transmitting and receiving optics and traverse system. a) Optics mounted on the tri-axis traverse. b) Mounted optics configured in back-scatter mode (Menchini, 2013).

A summary of the main system settings used for the PDPA data in Chapter 6 is listed in Table 3.1. The settings were configured for a dense water spray with input from colleagues who had experience in using the apparatus, and from the PDPA manufacturer. 
Table 3.1: Summary of PDPA system settings.

\begin{tabular}{|c|c|c|}
\hline Setting & Channel 1 & Channel 2 \\
\hline Laser Power $(\mathrm{W})$ & \multicolumn{2}{|c|}{3.34} \\
\hline Transmitter Focal Length (mm) & \multicolumn{2}{|c|}{1000} \\
\hline Slit Aperture $(\mu \mathrm{m})$ & \multicolumn{2}{|c|}{150} \\
\hline Min and Max Diameter $(\mu \mathrm{m})$ & \multicolumn{2}{|c|}{$2-685$} \\
\hline Lower-to-Upper Intensity Ratio & \multicolumn{2}{|c|}{0.1} \\
\hline Upper Intensity Slope $\left(\mathrm{mV} / \mu \mathrm{m}^{*} \mu \mathrm{m}\right)$ & \multicolumn{2}{|c|}{0.015} \\
\hline Focal Length (mm) & \multicolumn{2}{|c|}{1000} \\
\hline Measuring Volume Diameter $(\mathrm{mm})$ & \multicolumn{2}{|c|}{0.370} \\
\hline Measuring Volume Length (mm) & \multicolumn{2}{|c|}{8.77} \\
\hline Beam Wavelength (nm) & 514.5 & 488.0 \\
\hline Beam Separation $(\mathrm{mm})$ & \multicolumn{2}{|c|}{20} \\
\hline Laser Beam Diameter (mm) & \multicolumn{2}{|c|}{1.77} \\
\hline Beam Expander (ratio) & \multicolumn{2}{|c|}{2.11} \\
\hline Fringe Spacing $(\mu \mathrm{m})$ & 12.1947 & 11.5666 \\
\hline Beam Waist Diameter $(\mu \mathrm{m})$ & \multicolumn{2}{|c|}{3.7347} \\
\hline Bragg Cell Freq. (MHz) & \multicolumn{2}{|c|}{40} \\
\hline Velocity Limit Min (m/s) & -36.58 & -34.70 \\
\hline Velocity Limit Max (m/s) & 73.17 & 69.40 \\
\hline PMT Voltage (V) & 350 & 350 \\
\hline Max Number of Samples & \multicolumn{2}{|c|}{50,000} \\
\hline Time Out (sec) & \multicolumn{2}{|c|}{30} \\
\hline Burst Threshold for Both Channels (mV) & \multicolumn{2}{|c|}{30} \\
\hline SNR Setting & \multicolumn{2}{|c|}{ Low } \\
\hline Band Pass Filter (MHz) & \multicolumn{2}{|c|}{$1-10 M$} \\
\hline Down Mix Freq $(\mathrm{MHz})$ & \multicolumn{2}{|c|}{36} \\
\hline Software Coincidence (gate scale) \% & \multicolumn{2}{|c|}{$\begin{array}{c}250 \\
\text { Internal Reflection }\end{array}$} \\
\hline Scattering Mode & \multirow{2}{*}{\multicolumn{2}{|c|}{$\begin{array}{c}\text { Internal Reflection } \\
151\end{array}$}} \\
\hline Scattering Angle Off-axis (deg) & & \\
\hline Polarization & \multicolumn{2}{|c|}{ Perpendicular } \\
\hline Refractive Index of Droplet & \multicolumn{2}{|c|}{1.33} \\
\hline
\end{tabular}

\section{Section 3.3.1: Droplet Size Validation}

There are two methods for the system software to check the validity of the droplet size measurements: the intensity validation and the diameter difference validation. The intensity validation follows the principle that the intensity of light reflected by an individual particle should increase as the square of the diameter. Figure 3.4 a) is an example of the intensity validation with its operating settings listed in Table 3.1 configured with input from a representative of the PDPA 
manufacturer (Troolin, 2012). The second particle size validation takes advantage of the PDPA system's three photo detectors that recorded simultaneous dual particle sizing measurements. The difference in diameter was computed for the two simultaneous particle diameter measurements and particles with differences greater than $7 \%$ where filtered out. An example is shown in Figure 3.4 b). It should be noted that both of these methods also removed the measured velocity component data of the filtered drops.

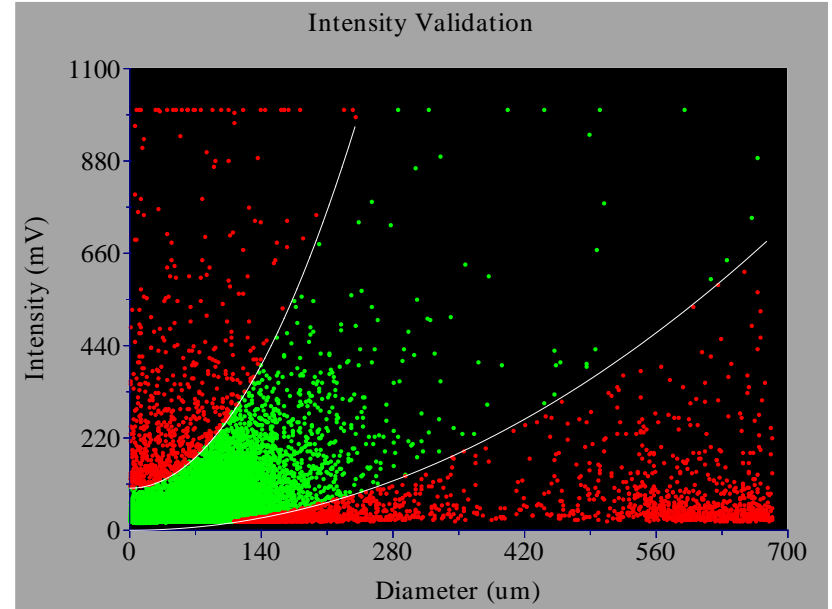

a)

Figure 3.4: Diameter data validation graphs. a) Intensity validation curve. b) Diameter difference validation (Hillen et al. 2013).

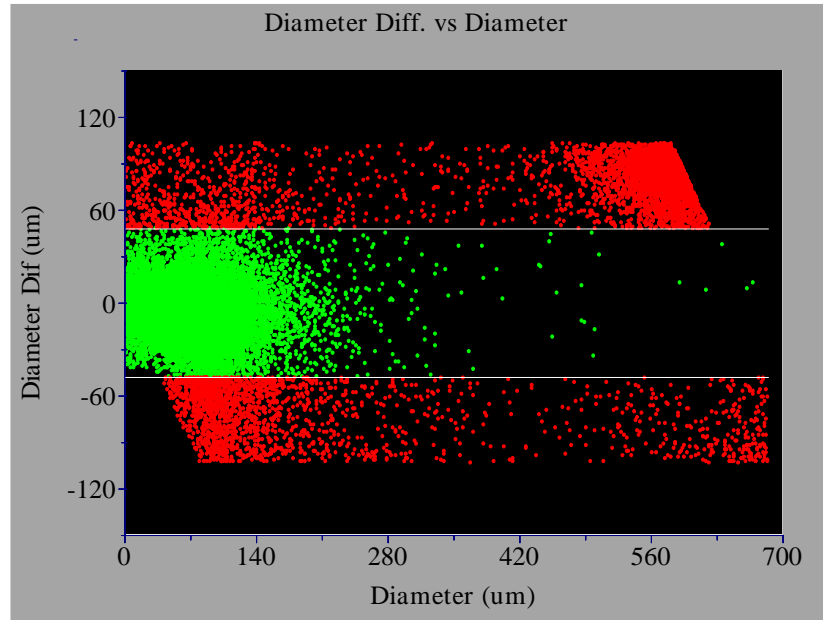

b) 
droplets. Defining the nozzle tip as the spray zero location $(x=y=z=0 \mathrm{~mm})$, initial sparsely space profiles along the $x$ and $y$-axis were recorded via PDPA to center the measuring volume.

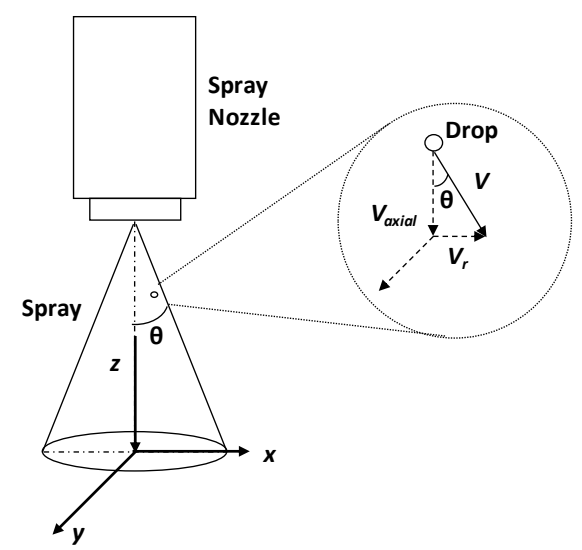

Figure 3.5: Coordinate system for spray axis and droplet coordinate system (Hillen et al., 2013).

Droplet velocity and size profiles were measured along the $x$-axis and $y$-axis at standoff distances from the nozzle tip of $z=3.18,3.81$, and $4.45 \mathrm{~cm}$. These distances were determined to be the optimal range needed such that a $5.08 \mathrm{~cm}$ diameter surface of interest would obtain full coverage with only a slight overspray, as shown in Figure 1.1. PDPA data were obtained at each nozzle standoff distance at 41 radial locations that were selected to yield equal annular ring area increments along the $x$-axis and $y$-axis for the three flow conditions listed in Table 3.3. The reasoning behind the equal area method was to simplify the volume flux calculations. These radial locations spanned the entirety of the spray $\left(-2.68 \leq R_{s} \leq 2.68 \mathrm{~cm}\right)$ for the $x$-axis profiles, but only two single point measurements were performed beyond the center of the spray in the $y$-axis before the very high spray density was believed to have affected the measurements. The pressures in Table 3.3 have been recorded just upstream of the nozzle where the manufacturer suggested 4.14 bar as one of the optimum operating conditions for the nozzle and 1.38 bar as on the low end to fully form a spray cone. Reliable constant pressures above 4.14 bar were not attainable, as the pump was unable to maintain steady operating conditions at the higher pressures. 
Table 3.3: Spray nozzle settings.

\begin{tabular}{|c|c|}
\hline Nozzle Gage Pressure (bar) & Flow Rate (L/hr) \\
\hline 1.38 & 29.5 \\
\hline 2.76 & 43.5 \\
\hline 4.14 & 54.5 \\
\hline
\end{tabular}

\section{Section 3.5: Splashed Droplets Methodology}

PDPA data was also obtained to characterize the splashed droplet sizes and speeds (Figure 3.2). The $5.08 \mathrm{~cm}$ diameter impact surface was mounted in the spray apparatus depicted in Figure 3.1 and aligned with the nozzle as described in Section 3.2.1 to generate the splashed spray droplets. The impact surface was positioned at the optimal standoff distance of $3.81 \mathrm{~cm}$ from the nozzle exit for full coverage and a slight overspray as in Figure 1.1. The PDPA was operated under the same system settings listed in Table 3.1 to characterize the splashed spray droplets with the measuring volume traversing along only the $x$-axis in the same location increments and $\operatorname{span}\left(-2.68 \leq R_{s} \leq 2.68\right.$ $\mathrm{cm}$ ) as described in Section 3.4. This profile was measured for each nozzle pressure and flow rate condition listed in Table 3.3 at a constant $z=3.65 \mathrm{~cm}$, or $1.6 \mathrm{~mm}$ above the impact surface. The selection of this standoff distance was based on many contributing factors, but the main consideration was to prevent the spray pedestal from interfering with the $x$ component incident laser beams depicted as a sketch in Figure 3.6. During testing it was observed that at lower flow rates there was a small ring of fluid buildup along the edge of the impact surface that could also interfere with the incident beams. This measuring volume $z$ location also provided ample clearance of the residual spray film thickness, which ranged nominally between 10 to $350 \mu \mathrm{m}$ as measured in the literature (see Table 2.3). Kalantari and Tropea (2007) suggested in their PDPA experiments that the measuring volume should be positioned at least $1 \mathrm{~mm}$ above the impact surface to avoid the maximum crown height and its jet formations, which they computed analytically. 


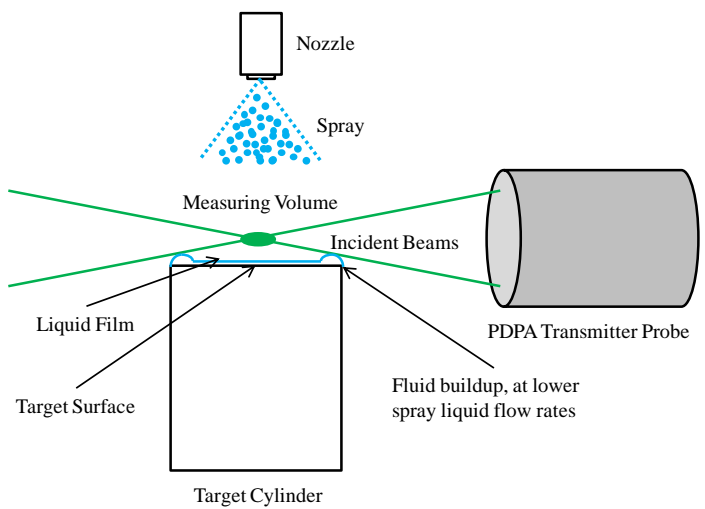

Figure 3.6: Sketch explaining the $z$ positioning of the measuring volume to avoid interference from the impact surface and fluid ring buildup.

The PDPA data acquisition software did not discriminate splashed droplets from impinging spray droplets. As a result, a method had to be developed to extract the splashed droplet sizes and speeds. The developed technique separated the data in greater detail than the method used by Kalantari and Tropea (2006) that was summarized in Section 2.3.3. Figure 3.7 a) depicts the raw PDPA spray droplet population data for one condition and location in the spray with no impact surface in place. Figure 3.7 b) shows the raw PDPA droplet population data under the same spray conditions and measuring location, but with the impact surface in place. Examining Figure $3.7 \mathrm{~b}$ ) shows a similar spray droplet population, but with the addition of a secondary population formation to its left that was not present in Figure 3.7 a). This additional population was determined to be the splashed droplets since they were significantly slower with a large portion having the opposite axial velocity sign convention relative to the original spray droplets, and since this population only existed for measurements collected with the impact surface in place. 


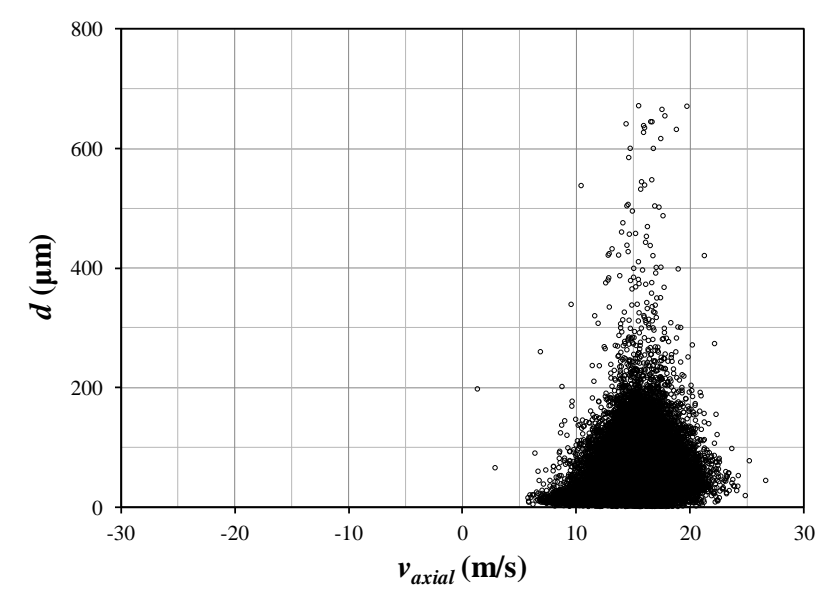

a)

Figure 3.7: Comparison of raw PDPA spray data of droplet diameter plotted vs. axial velocity. a) $R_{s}=0, z=3.2 \mathrm{~cm}$ for the 2.76 bar spray without the impact surface, b) $R_{s}=0, z=3.2 \mathrm{~cm}$ for the 2.76 bar spray with the impact surface.

The spray and splashed droplet populations shown in Figure 3.8 are categorized into four sub groups: spray, ejected splash, return splash, and absolute splash. The spray droplets always had positive axial velocities that were significantly higher than the corresponding splashed droplets, thus this population always formed to the right of the splashed droplet population in the plots of drop diameter versus axial velocity. The splashed droplets circled in green in Figure 3.8 were divided into the ejected and return splash droplet sub groups based on the axial velocity sign convention. Droplets in the splash region with a negative axial velocity (away from impact surface) were the ejected splash droplets, and droplets in the splash region with a positive axial velocity (returning to the surface due to gravity) were denoted as the return splash droplets. In addition, data have been presented for the splashed particles based on the absolute axial velocity magnitude consisting of the entire splash region termed herein as the absolute splash droplets. Data shows a double peak distribution and the dividing line between the two populations is the minimum between the two peaks. A sensitivity study for the effects of the location of the separation point on the averaged data for the different spray and splash parameters was performed. This study found that any reasonable 
location choice within a nominally 3 to $5 \mathrm{~m} / \mathrm{s}$ range had a negligible effect on the average of droplet data.

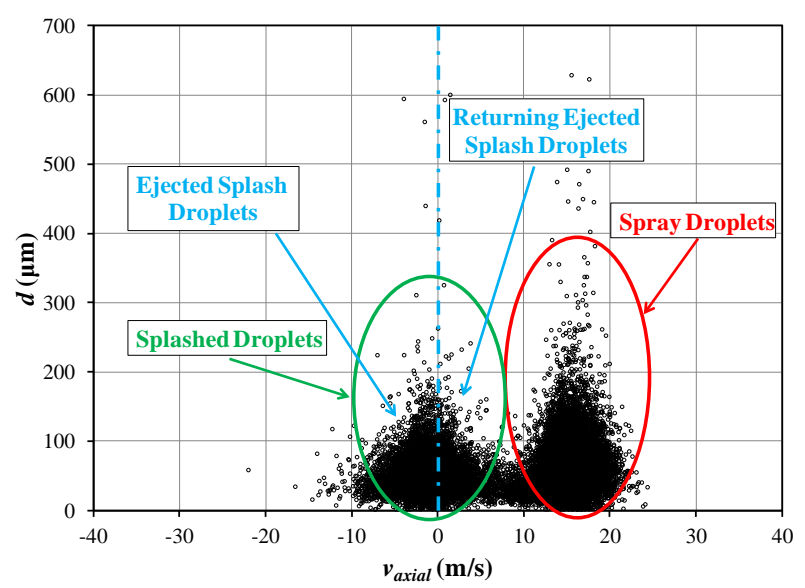

Figure 3.8: Separation of the spray and splash regions into the droplet sub-categories.

\section{Section 3.5.1: Vertical Edge Splash Droplet Methodology}

The addition of the splashed droplets created larger droplet number fluxes for the PDPA to interpret for the $x$-axis profiles above the impact surface. This resulted in lower data rates than corresponding measurements without the spray impact surface in place and was most prominent at higher nozzle pressures. Figure 3.9 shows evidence of the data rate decreasing with increasing nozzle pressure for PDPA data collected at $R_{s}=0 \mathrm{~cm}$ and $2.1 \mathrm{~mm}$ above the impact surface $(z=3.6$ $\mathrm{cm}$ ) at equal measuring times. The total droplet diameter counts for increasing the pressure are 27,295, 14,832, and 4,245 for the respective Figures 3.9 a), b), and c). This drop in diameter measurements shows that denser sprays negatively affect the quality of the PDPA data. 


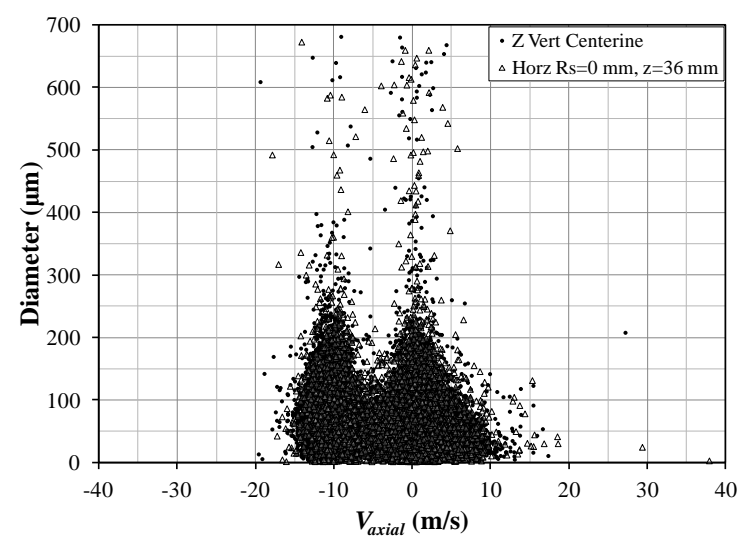

a)

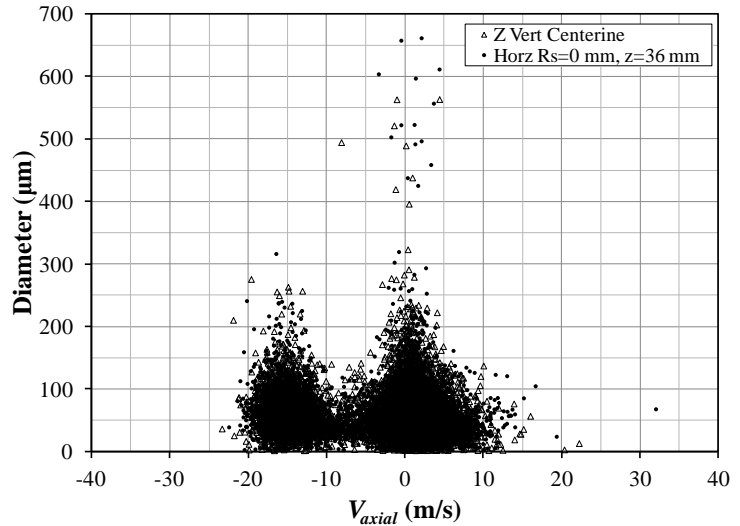

b)

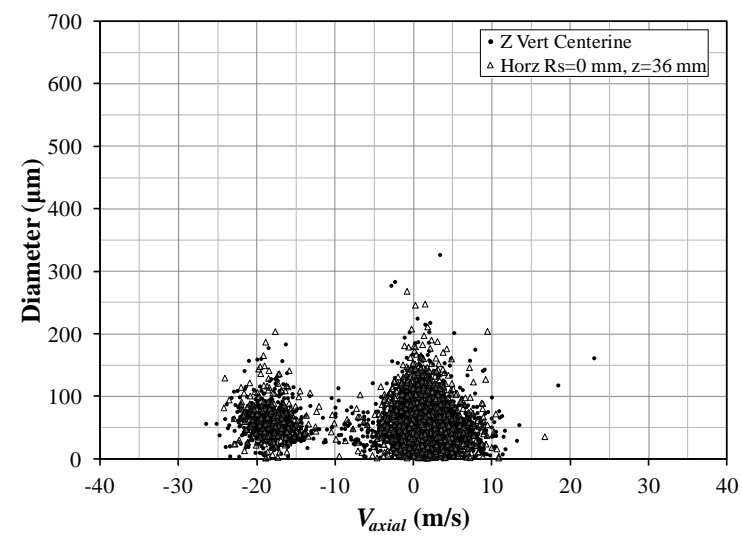

c)

Figure 3.9: Spray and splash droplet diameters versus axial velocity at $z=3.6 \mathrm{~cm}$ at the three nozzle flow conditions. a) 1.38 bar and $29.5 \mathrm{~L} / \mathrm{hr}$. b) 2.76 bar and $43.5 \mathrm{~L} / \mathrm{hr}$. c) 4.14 bar and 54.5 L/hr.

Also adversely affecting the PDPA splashed data quality was a smaller population on which to base the statistics. This is a result of the splashed droplet data being extracted from a finite amount of overall PDPA data (maximum of 50,000 droplet data per measurement location). Figure 3.10 clearly demonstrates this issue for the sample incoming spray, and splashed droplet diameter histograms at the center of the spray. The absolute splashed droplets histogram in Figure $3.10 \mathrm{c}$ ) shows the largest diameter bin counts, of nominally 60 to 70, for histogram depicted in Figure 3.10. 


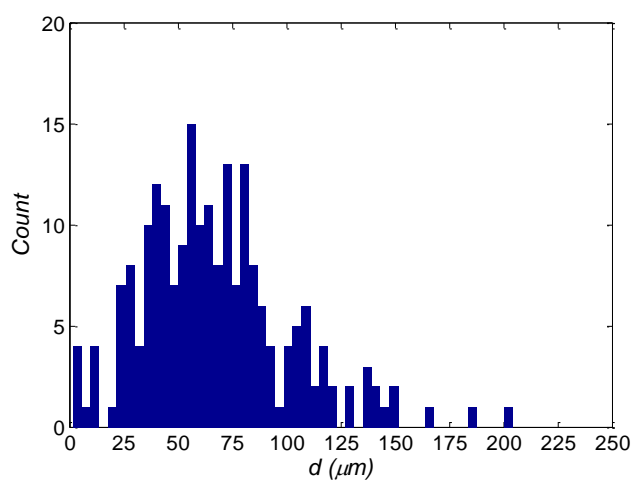

a)

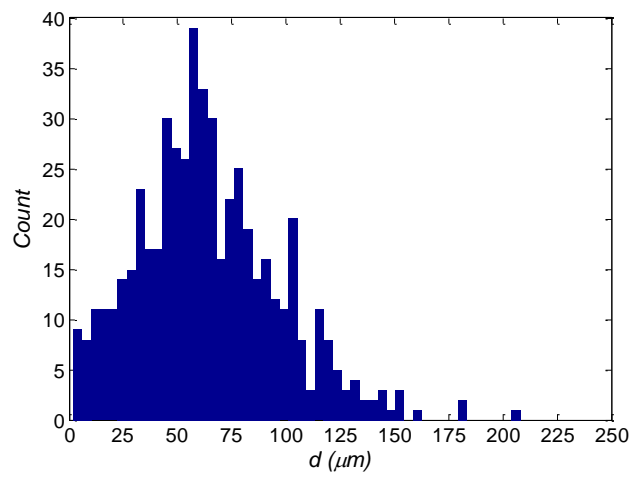

c)

Figure 3.10: Histograms of the separated droplet diameter data for a spray at 4.14 bar impinging on impact surface at $R_{s}=0 \mathrm{~cm}$ and $z=3.65 \mathrm{~cm}$. a) Spray droplets. b) Ejected splashed droplets. c) Returning splashed droplets. d) Absolute splashed droplets.

The lower data rates combined with less available splash droplet statistics prompted the investigation of the splashed droplets in a region with minimum interference from the impinging spray for comparison purposes. This was accomplished by performing measurements along the $z-$ axis of the ejected splash droplets just beyond the outer edge of the spray, as is highlighted in Figure 3.11. In this region post processing was not necessary to extract the splash data since the droplets were predominantly splash products. Measurements were obtained for vertical profiles on the $x z-$ plane at $y=0$ spanning between $1.75 \leq z \leq 4.25 \mathrm{~cm}$ at a fixed $R_{s}=2.68 \mathrm{~cm}$ for the spray conditions in Table 3.3. This profile allowed measurements just beyond the target pedestal at $z$ coordinates that were located both above and below the plane of the impact surface. The same vertical traverses under the same spray conditions were measured for $R_{s}=-2.68 \mathrm{~cm}$ to check for symmetry. 


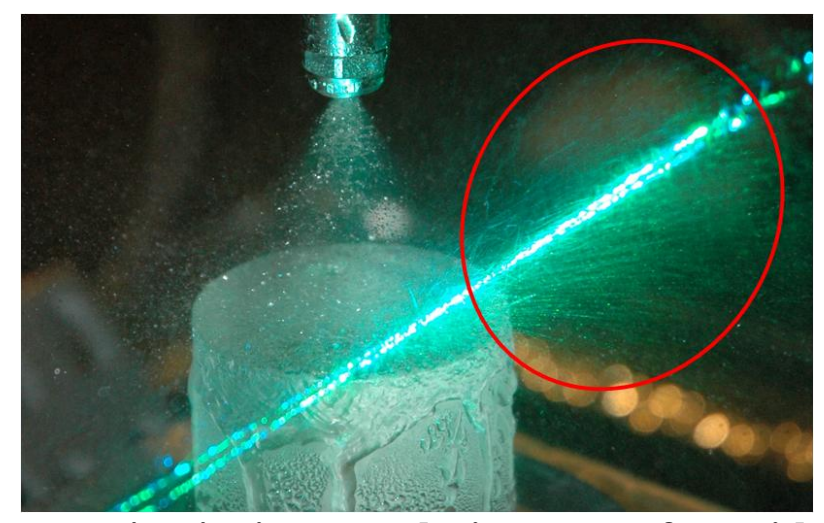

Figure 3.11: Spray apparatus impinging onto the impact surface with the laser probe volume traversing along the $z$-axis to the side. The splashed secondary droplets ejected outside of the spray cone that are being measured are circled in red. 


\section{CHAPTER 4: $\quad$ Single Drop EXPERIMENTS APPARATUS AND ProcedURES}

\section{Section 4.1: Introduction}

This chapter documents the experimental apparatus and procedures for the single droplet experiments, which were originally reported in Hillen et al. (2012), and Hillen et al. (2013). The single droplet experiments consisted of measuring the sub-cavity liquid film thicknesses via the CHR sensor along with simultaneous high-speed videos for flow visualization for the appropriate ranges

of $W e, R e$, and $h_{0}{ }^{*}$. The methodology for obtaining the transverse profiles and traversing the cavity to record the radial film thickness history profiles are reported herein. The radial profiles of the subcavity film thickness were utilized in the sub-cavity volume calculation method, as presented in Chapter 5.

The single drop apparatus is discussed in Section 4.2 including the droplet generator, optical probe housing, and traverse assembly. Descriptions of the noncontact CHR sensor utilized to measure the sub-cavity film thickness, and the high-speed video camera set up used for detailed flow visualization are also given in this section.

Section 4.3 documents the preliminary investigations of the sub-cavity single drop experiments found in Hillen et al. (2012). The initial methodology of measuring the crown radius and height versus time is discussed. The original methodology of utilizing the maximum crown radius to determine the radial location to traverse the cavity is also detailed.

For the main single drop experiments, the bottom inside cavity radius, referred herein as the sub-cavity radius, was determined to be the most effective method to determine the radial locations at which to traverse the CHR optical probe. Section 4.4 contains a detailed description of the two high-speed video techniques used to measure the sub-cavity radius versus time. The maximum subcavity radius was utilized to generate the traverse limits for the CHR optical probe. Also included in Section 4.4 are the image processing techniques to extract the sub-cavity radius values. 
Section 4.5 contains the procedures for producing the desired single drop test conditions and measuring the sub-cavity liquid film thickness histories with the CHR sensor. In addition the methodology for creating the radial cavity traverse profiles for the CHR optical probe is documented. Post processing procedures for correcting the recorded film thicknesses and timing are also reported. A brief summary of the image processing code developed to obtain the single droplet diameter, velocity, and impact location is reported.

\section{Section 4.2: Single Drop Sub-cavity Experiments Apparatus}

The apparatus used to measure the sub-cavity film thickness histories for the single drop experiments is depicted schematically in Figure 4.1. Single droplets of the desired size have been produced via a combination of solenoid valve total opening time, pressure head in the generator's liquid reservoir, and the needle orifice opening in which droplets detach due to gravity. The presented single drop experiments incorporated a 12-gauge stainless steel precision tip dispensing needle with a $2.16 \mathrm{~mm}$ inside diameter orifice. The droplet generator was attached to a vertical track allowing the droplet velocity prior to impact to be varied by changing the free fall height of the droplet. Generated droplet diameters and velocities had measured standard deviations of nominally $0.015 \mathrm{~mm}$ and $0.007 \mathrm{~m} / \mathrm{s}$ respectively for ten subsequent droplets. The individual droplets impinged onto a static layer of liquid contained within a nominally $15.2 \times 15.2 \times 5.1 \mathrm{~cm}$ optically clear acrylic tank open to the atmosphere. The tank size was deemed adequate to allow surface waves to damp before wall rebound.

Liquid film thickness has been measured with a time resolved non-contact chromatic optical CHRocodile SE sensor using a $3.3 \mathrm{~mm}$ full range optical probe. The sensor functions by focusing spectrally broadband light at an interface from which it is reflected back to the sensor. For thickness measurements, the sensor interprets two interfaces at different distances as two sharp peaks in the 
wavelengths. The spectral distance combined with the refractive properties of the interfaces result in thickness measurements (Kunkel and Schulze, 2005). The sensor had a sampling rate of $4 \mathrm{kHz}$ and $\pm 0.1 \mu \mathrm{m}$ thickness sensitivity. It was observed that the sensor could reliably read slightly beyond the nominal $3.3 \mathrm{~mm}$ range, to between $3.4-3.5 \mathrm{~mm}$. The optical probe was housed within an impact stand covered with a $0.152 \mathrm{~mm}$ thick, $25 \mathrm{~mm}$ diameter borosilicate glass disc that served as the interface between the sensor and the liquid pool as depicted schematically in Figure $4.1 \mathrm{~b}$ ). Film thickness data acquisition was performed using the manufacturer's CHRView software. The impact stand was fitted in the middle of the acrylic tank resulting in the $10.16 \mathrm{~cm}$ diameter surface that functioned as the wall, referred herein as the impact surface. The tank and all of its components were mounted on a single axis micro-traverse allowing sub-cavity thickness profiles to be measured at locations located radially away from the fixed droplet impact centerline. The micro-traverse had a $0.025 \mathrm{~mm}$ resolution. The impact surface was leveled via a leveling plate attached to the microtraverse. Detailed computer aided design (CAD) drawings of some of the major components can be found in Appendix A.
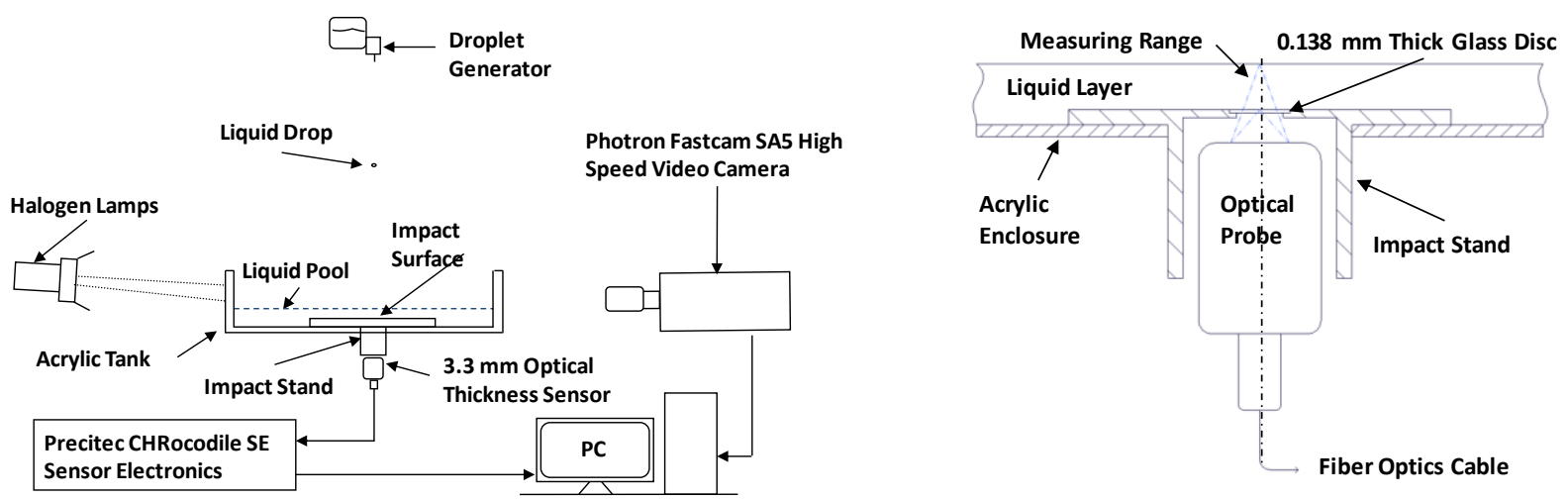

a)

b)

Figure 4.1: Schematics of the single drop experimental apparatus. a) Entire apparatus. b) Cross section of the impact stand and optical probe (Hillen et al. 2012).

Droplet impacts were recorded utilizing a gray scale Photron FASTCAM SA5 high-speed video camera equipped with a Nikon ED AF MICRO NIKKOR $2000 \mathrm{~mm} \mathrm{1:4} \mathrm{D} \mathrm{lens} \mathrm{for} \mathrm{detailed}$ 
analysis. The camera was capable of recording images at a maximum 150,000 frames per second (fps) at $128 \times 32$ pixel resolution. It had a maximum resolution of 1024 x 1024 pixels up to $7500 \mathrm{fps}$. For the sub-cavity film thickness experiments the camera's line of sight was aligned parallel to the impact surface in a backlit configuration shown in Figure 4.1 a). The camera was illuminated with a 250-W Lowel Pro halogen lamp positioned at the rear of the tank. A camera frame rate of 12,000 fps, 640 x 640 pixels was required to adequately capture the impact event and to prevent washout from the light source. Using the proprietary Photron FASTCAM Viewer software, a high-speed image of a caliper set to $10 \mathrm{~mm}$ was used to calibrate the pixel sizing. Depending on the distance of the camera from the tank, typical backlit configurations resulted in pixel resolutions of $0.0441 \mathrm{~mm}$ to $0.0476 \mathrm{~mm}$. A control switch was used to simultaneously trigger the camera during droplet generation and synchronized with the CHR sensor time while it recorded the liquid layer thickness.

\section{Section 4.3: Initial Preliminary Investigation}

Initial preliminary development of the current methodology utilizing the apparatus described in Section 4.1 to map the sub-cavity film thickness has been documented in greater detail in Hillen $e t$ al. (2012). Figure 4.2 is a sketch with definitions of a typical history of the sub-cavity liquid film thickness histories where the cavity lifetime is defined to begin upon droplet impact.

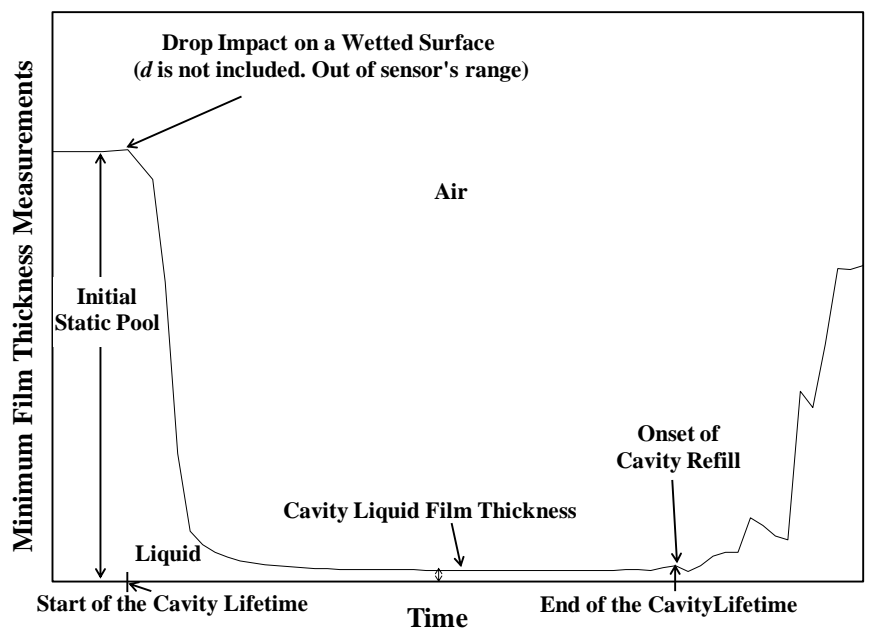

Figure 4.2: Definition of a cavity lifetime for a wetted surface, (Hillen et al., 2012). 
Two single drop impingement cases listed in Table 4.1 were studied in the preliminary investigation using distilled water as the working fluid (see Table 3.2 for properties). The low Weber number and medium initial liquid film thickness were chosen for Case A to investigate a low momentum drop impact below the Cossali et al. (1997) splashing criteria in Table 2.2. Case B parameters were based on a single drop impingent condition used in simulations by Nikolopoulos $e t$ al. (2007) for comparison purposes; this case is above the splashing threshold.

Table 4.1: Dimensional and corresponding dimensionless parameters for preliminary single drop cavity investigation (Hillen et al., 2012).

\begin{tabular}{|c|c|c|c|c|c|c|c|}
\hline Case & $\boldsymbol{h}_{\boldsymbol{0}}{ }^{*}$ & $\boldsymbol{d}(\mathbf{m m})$ & $\boldsymbol{v}(\boldsymbol{m} / \mathbf{s})$ & $\boldsymbol{W e}$ & $\boldsymbol{R e}$ & $\boldsymbol{F r}$ & $\boldsymbol{Y}$ \\
\hline $\mathrm{A}$ & 0.614 & 3.19 & 1.79 & 141 & 5,700 & 103 & 0.398 \\
\hline $\mathrm{B}$ & 0.113 & 6.39 & 2.69 & 633 & 17,100 & 115 & 3.50 \\
\hline
\end{tabular}

The film thickness lifetimes had to be manually determined during post processing with the aid of high-speed flow visualization. Due to a timing error between the CHR sensor and the highspeed camera, the beginning of the sub-cavity liquid film thickness profiles located off the centerline had to be manually corrected by utilizing the bottom crown radius propagation. The measured crown dimensions were also used for comparisons between experiments and corresponding CFD simulations as presented in Hillen et al. (2012). In Figure 4.3 the bottom crown radius is defined as the half the distance between the opposite outside edges of the crown and the free surface interface. Crown height was defined as the distance from the crown-free surface interface to the base of the fingering, as also shown in Figure 4.3. Defining the crown height in this manner eliminated any judgment required from trying to measure to a specific point in the fingers, which vary from drop-todrop. 


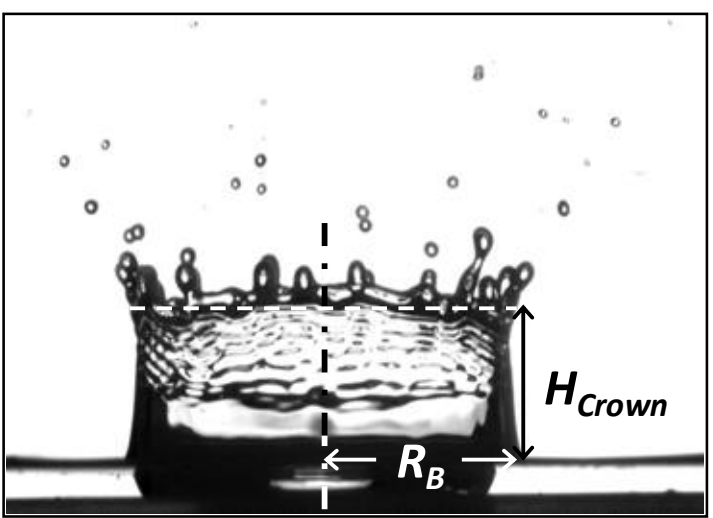

Figure 4.3: Definition of crown height and bottom radius.

Bottom outside crown diameters and crown heights were extracted from the high-speed videos via a custom automated image processing code. This code was developed in Matlab, for its efficient matrix manipulation routines and to take advantage of the built in Image Processing Toolbox. For the program to efficiently run, the frames of the beginning of the crown formation and its full collapse first had to be inputted. Once the range of frames of interest have been defined, the code extracts an average pixel location of the liquid free surface from an image of the undisturbed free surface. Subsequent video frames are uploaded in sequence and converted into binary (black and white) images to make subsequent image processing simpler. Crowns in subsequent frames were outlined by employing a built in Canny edge detection algorithm. Any holes in the crown outline are connected and the crown and liquid film are filled. Any splashed droplets and noise in the filled image were filtered out. The free surface and corresponding liquid film beneath the crown were then removed with the previously stored undisturbed free surface pixel coordinates. Crown fingers were then removed through the following process. The image was scanned to identify the first and last pixel in each row, removing any rows that contain any black pixels between these pixels. The removal of these rows removes the crown fingers leaving a smooth upper crown surface at the lowest point in the observed cusps. This results in the measuring range from which the crown height 
and bottom crown radius are extracted. Figure 4.4 presents the steps used to extract the crown height and bottom crown radius from the high speed video images.

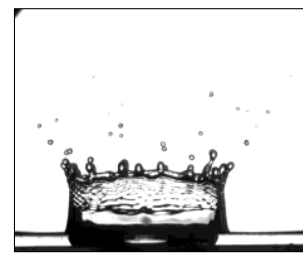

Original Image

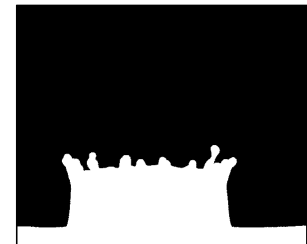

Filled and Filtered Image

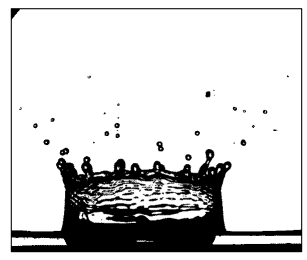

Binary Image

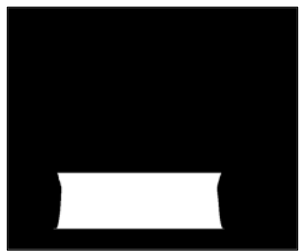

Measurement Boundary

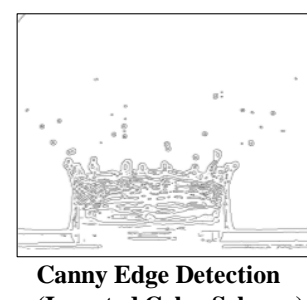

(Inverted Color Scheme)

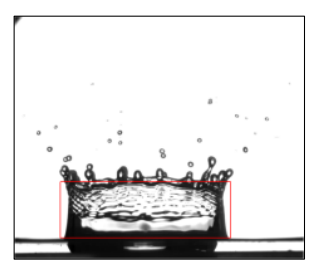

Bounding Box of Measuring Range

Figure 4.4: A basic overview of the Matlab image processing used to determine the crown height and bottom crown radius. The order of the sequence is left to right from the top (Hillen et al., 2012).

\section{Section 4.4: Single Drop Sub-cavity Radius Video Methodology}

The CHR traverse profiles created from the procedure described in Section 4.3 resulted in a low number of sub-cavity film thickness radial profiles, which is explained in more detail in Section 7.2. Thus the sub-cavity radius (see Figure 1.2) was determined to be the most effective method to determine the radial locations at which to traverse the cavity with the CHR optical probe. Therefore, two different high-speed video techniques have been developed to measure the inside sub-cavity radius for a single drop impinging on an initial thin liquid film. These two techniques are herein referred to as the side-view (SV) and bottom-view (BV) methods, based on their respective camera configurations. The camera alignment for the SV method was the same backlit configuration depicted in Figure 4.1 a). Images were acquired at 12,000 fps, 640 x 640 pixels as described in Section 4.2. Because the sub-cavity radius was of primary interest the camera height was adjusted 
such that its line of sight was level with the impact surface to provide greater visual clarity of the cavity formation.

The initial investigation into the development of the optimum bottom-view methodology has been reported in Hillen and Kuhlman (2013b). The bottom-view method involved modifications to the single drop apparatus shown in Figure 4.1 a) in order to visualize the impact process from beneath a transparent impact surface. This was accomplished by positioning an acrylic mirror beneath the impact stand. The mirror and high-speed camera were oriented such that the line of sight was perpendicular to the impact surface, as depicted schematically in Figure 4.5. To ensure adequate visualization of this phenomenon, a similar impact stand to the one depicted in Figure $4.1 \mathrm{~b}$ ) was constructed, but it contained a larger $4.45 \mathrm{~cm}$ diameter viewport with a 48 x 60 x $0.15 \mathrm{~mm}$ rectangular borosilicate slip glass covering it (this impact stand was exclusive to the BV bottom cavity measurements). Due to less available light, the BV videos were recorded at 1,000 fps with $1,024 \times 1,024$ pixel resolution. Each pixel had a typical resolution of between $0.0570 \mathrm{~mm}$ to 0.0656 mm depending on the camera positioning.

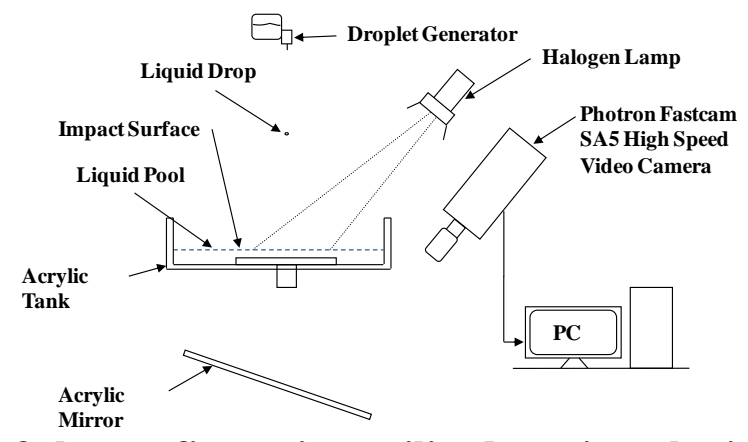

Figure 4.5: Schematic of the configuration utilized to view the impingement process from beneath the free surface (Hillen and Kuhlman, 2013b).

The CHR sensor was utilized to obtain the correct initial film thickness based on the single drop test condition for both sub-cavity radius video techniques. The CHR optical probe was removed prior to testing for the BV technique to create an unobstructed view. The sub-cavity radius was measured versus time, and analyzed using custom image processing codes presented in Section 
4.4.1. Figures 4.6 a) and b), respectively, are example images from the SV and BV videos for a drop impingement condition of $W e=140, R e=1,210$, and $h_{0}{ }^{*}=1.0$. Indicated in red in each image is the measured bottom cavity diameter for the time shown.

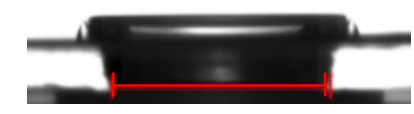

a)

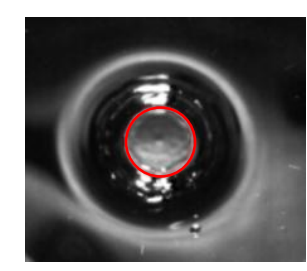

b)

Figure 4.6: Example images of the measured sub-cavity radius. a) Side-view image of measured sub-cavity radius (red line indicates measured diameter). b) Bottom-view image of measured sub-cavity radius (red circle indicates measured diameter) (Hillen et al. 2013).

\section{Section 4.4.1: Sub-cavity Radius Image Processing}

Two separate automated Matlab based image processing codes were developed to measure the sub-cavity radius histories for the SV and BV videos. Figure 4.7 depicts a basic overview of the logic used for the side-view code. The first step of the process was to identify the liquid layer region by manually imputing the average pixel location of the impact surface and the bottom of the free surface using a frame consisting of the undisturbed initial film thickness. For simplification of the process, each subsequent frame was converted into a binary image after which pixels not located within the liquid layer region were removed. A Canny edge detection function was again used to outline the remaining cavity, which was subsequently filled and filtered by applying various methods to remove noise. The bottom inside cavity diameter was averaged for the lowest five rows of pixels consisting of the bottom cavity. This reduced noise during the initial cavity depth propagation due to its large curvature, and noise at the bottom cavity and impact surface interface due to minute shadows. 

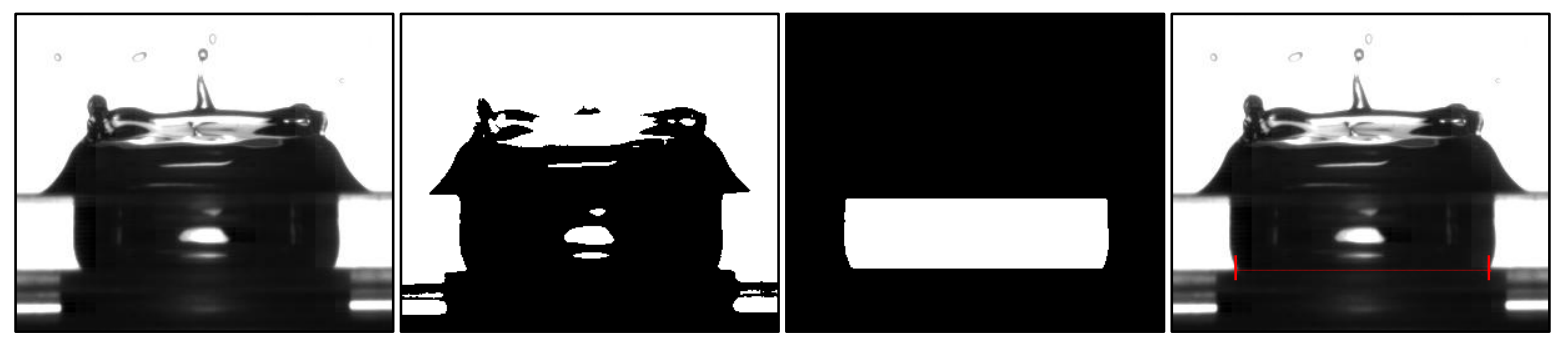

Figure 4.7: A basic overview for the side-view video image processing. From left to right: original image, binary conversion, filled cavity of the Canny edge detection outline, and the final measured bottom cavity diameter.

The code developed for the bottom-view sub-cavity radius measurements follows a similar initial logic used in the SV version. Figure 4.8 is a basic summary of the BV code logic, where the initial step was to convert subsequent frames to binary images. The cavity was then filled for simplification. The positioning of the light source created glare spots in the crown preventing the cavity region from being isolated, as shown in Figure 4.8. To rectify this issue the code searched for the maximum distance between the available inside cavity edges and connected the glare spots based on this distance and the user defined cavity center. Once the cavity region was enclosed the remaining pixels were filtered out leaving the isolated cavity. Cavity diameter and centroid were then measured utilizing the built in Matlab function "imfindcircles."
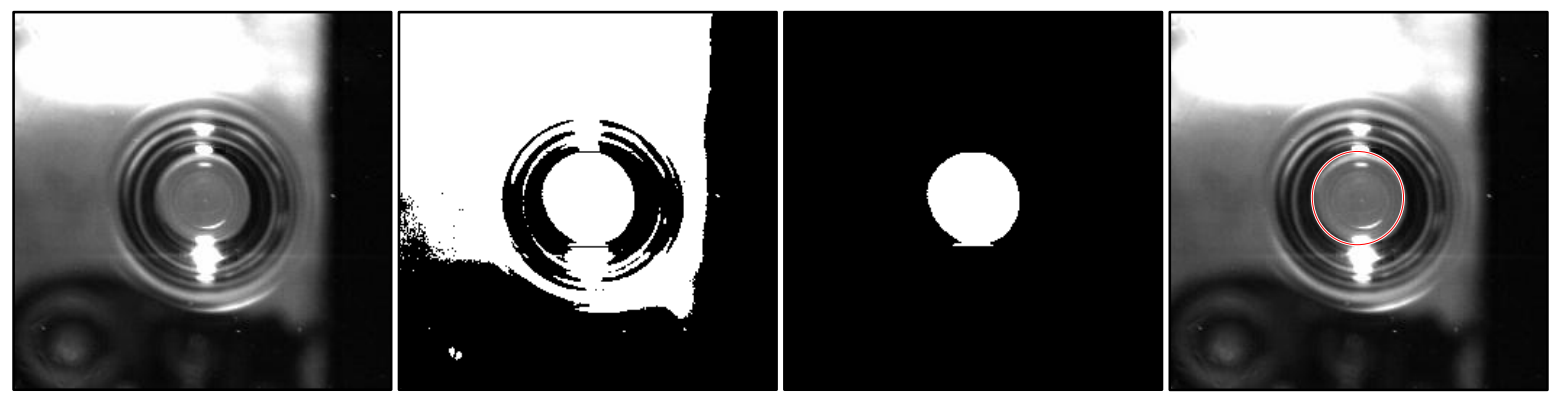

Figure 4.8: A basic overview for the bottom-view video image processing. From left to right: original image, filled binary conversion with glare spots connected, isolated cavity, and the final measured bottom cavity diameter.

\section{Section 4.5: Single Drop Sub-cavity Methodology}

The initial experimental procedures for measuring the single drop sub-cavity film thickness histories were originally described in Hillen et al. (2012), with the updated, improved process 
documented by Hillen et al. (2013). For the results presented in Chapter 7, the initial droplet diameters and velocities were varied nominally between $3-3.5 \mathrm{~mm}$, and $1.7-4.2 \mathrm{~m} / \mathrm{s}$ respectively to match a specific We and Re combination in the desired test plan described in Section 6.5. Individual droplet size, impact velocity, and impact location were extracted from the corresponding high-speed video with custom image processing detailed in Section 4.5.1. The measuring point of the CHR optical probe was aligned with the averaged location of droplet impact as measured using high-speed imagery. This measuring point is considered the CHR sensor zero location. Liquid was added to the tank rather than removed to obtain the desired initial film thickness since this resulted in the most significant reduction in the tank wall meniscus. The high-speed video camera was set up in a backlit configuration as depicted in Figure 4.1 with the height of the camera line of sight adjusted so that the free surface would be clearly distinguishable. Each droplet was triggered individually with a programmed 6 second trigger delay between runs that ensured ample time for the free surface to reach steady state after each impact.

The radial measuring points used to traverse the CHR optical sensor was constructed with the maximum sub-cavity radius data obtained from the SV analysis, using the process detailed in Section 4.4. The SV cavity radius data were generally somewhat larger than the respective BV data, thus ensuring full coverage of the bottom cavity history data obtained with the CHR sensor. The utilization of the maximum sub-cavity radius resulted in profiles that contained at least 8 to 14 locations at which film thickness data were recorded. The equal area annular ring method was used to distribute the radial measuring locations, so that a finer radial resolution resulted at the outer edges of the cavity, as shown schematically in Figure 4.9. This focus on the outer radial locations was an attempt to more accurately capture the location where the cavity bottom ended. Small uncontrollable air currents in the lab and the uncertainty in the droplet detachment process from the needle resulted 
in a variability in the drop-to-drop impact location. This variability in the droplet impact location determined the minimum meaningful radial step size and thus the number of traverse points for each test scenario. For the range of initial drop conditions presented in Section 7.3 this uncertainty in impact location increased from $4 \%$ of the initial droplet diameter at the lowest free fall height to $23 \%$ of the initial droplet diameter at the highest droplet free fall height (corresponding to the highest momentum case studied).

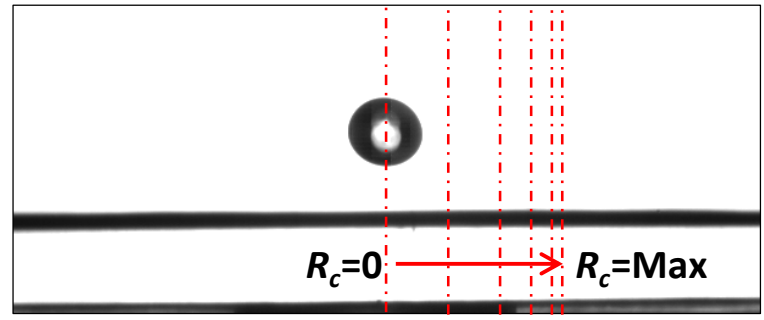

a)

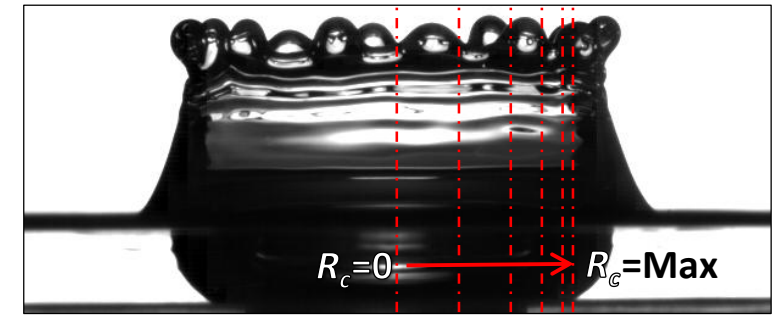

b)

Figure 4.9: Schematic representation of the radial cavity traverse locations for the CHR optical probe (Hillen et al. 2013).

The optical probe was traversed radially outward from the droplet impact centerline to each of the specified maximum radial locations. This was done by fixing the location of the droplet generator, and traversing the droplet impact tank (see Section 4.2). Individual droplet impact data were recorded for each radial location, maintaining consistent initial droplet impact location and film thickness conditions for the specified case. Droplet impacts which varied more than $1 \%$ away from the impact centerline location were excluded from the analysis with aid of the SV videos. This was accomplished using the Photron FASTCAM Viewer software to estimate the average droplet impact pixel location in the videos. The shape of the $1.27 \mathrm{~cm}$ diameter view port in the acrylic stand for the CHR probe light to pass through was visible in the backlit videos, as highlighted by the red box in Figure 4.10. Assuming perfect probe alignment, the droplet impact centerline location should be located in the center of this viewport. This location could be determined to within one pixel by subtracting the distances from the left side of the image to the two sides of the viewport and dividing 
by two. Adjusting the lateral position of the acrylic tank and the droplet generator until both the average droplet impact location and the viewport were centered in the image ensured that the optical probe measuring point was aligned with droplet impact centerline. Thus, the droplet impact centerline was defined to within one consistent pixel location (nominally $0.0570 \mathrm{~mm}$ to $0.0656 \mathrm{~mm}$ ) for videos for all radial traverse locations for a particular camera alignment.

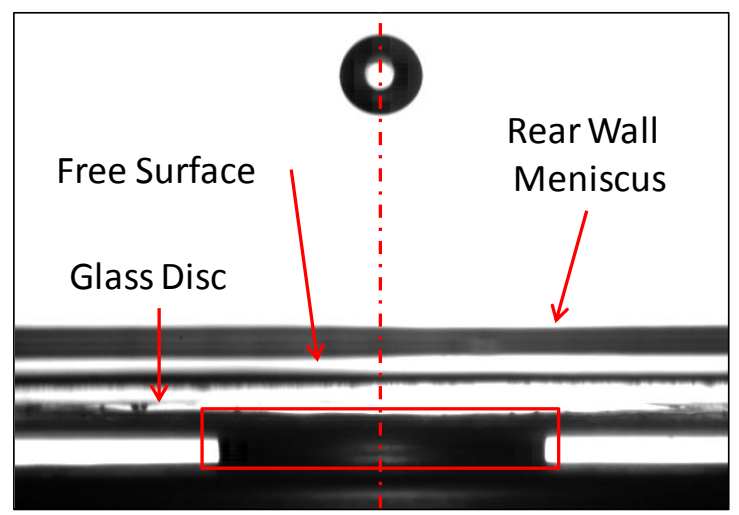

Figure 4.10: Example determining the CHR optical probe measurement center point in the videos with the probe positioned at $R_{c}=0 \mathrm{~mm}$. The red box highlights the $1.27 \mathrm{~cm}$ diameter acrylic hole "viewport" and the red dashed line represents the probe's center point.

Timing and film thickness corrections for the sub-cavity profiles were performed during post-processing. The cavity lifetime has been defined to start when the droplet first comes into contact with the free surface. This proved trivial for the impact centerline measurements, as the film thickness data abruptly changed when the droplet contacts the free surface creating one interface. Corresponding synchronized high-speed videos of the impinging drop allowed the time corrections for thickness profiles where $R_{c}>0$ to be within $\pm 0.33 \mathrm{msec}$ of the inter-frame time of the video. To properly account for the thickness of the glass interface, the index of refraction of the CHR sensor was set to a value of one so the true liquid film thickness was extracted by the following equation (Hillen et al., 2013):

$$
h_{\text {Fluid }}=\left(h_{\text {Total }}-\frac{h_{\text {Glass }}}{\eta_{\text {Glass }}}\right) \eta_{\text {Fluid }}
$$




\section{Section 4.5.1: Single Droplet Image Processing}

Single droplet diameter, velocity, and impact location were measured for each impact in the high-speed videos via an automated Matlab-based image processing code. The user would input a frame number range between when the droplet first fully comes into view and before it passed over the meniscus, or free surface. Subsequent frames were again converted to binary to simplify the code and all pixels where removed other than those consisting of the droplet. The isolated droplet was outlined through the Canny edge detection and the outline subsequently was filled. An ellipse was fitted to the drop since it would oscillate while falling, therefore the extracted droplet diameter was taken as the average of the two ellipse axes. The droplet centroid location from left edge of the image was taken as the center of the ellipse. Velocities were computed from the distance traveled by the droplet centroid for each subsequent frame. The final droplet impact diameter and locations were averaged over the last twenty frames until impact while the impact velocity was averaged for the last ten frames.
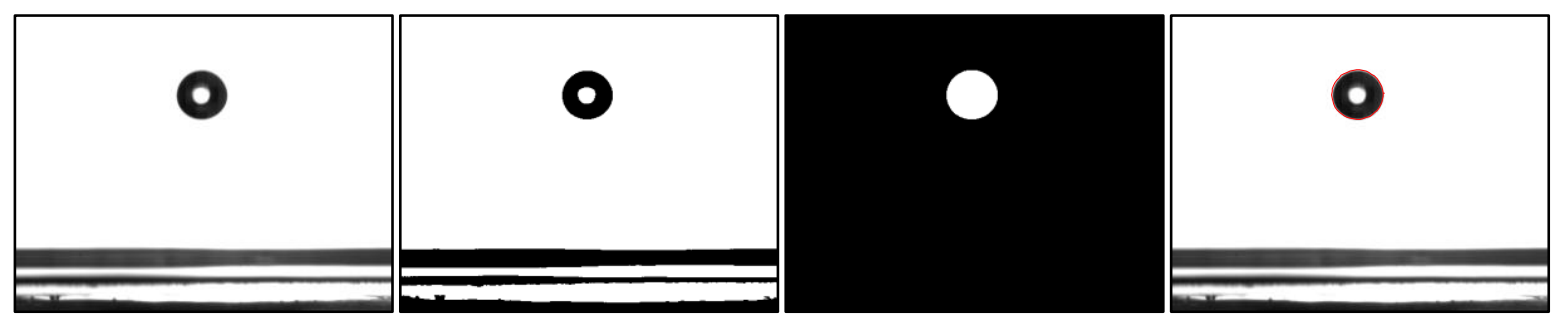

Figure 4.11: A basic overview of the single drop image processing. From left to right: original image, binary conversion, isolated filled drop, and the final measured drop diameter. 


\section{CHAPTER 5: Single Drop Sub-Cavity Volume Calculation METHODOLOGY}

\section{Section 5.1: Introduction}

The present chapter explains the methodology developed to compute the sub-cavity volumes for the single droplet experiments, as originally documented by Hillen et al. (2013). Both the preprocessing of the liquid film thickness histories to apply to the sub-cavity film thickness surface plots and subsequent sub-cavity volume calculations will be discussed in detail. Also, the integration method and iterative process used to determine the final sub-cavity volume histories are documented. The volume calculations for the presented methodology were automated via Matlab. A brief comparison of the sub-cavity volume results computed using the developed numerical method with an estimation utilizing the average minimum sub-cavity film thickness and measured sub-cavity radius history is also included.

\section{Section 5.2: Sub-cavity Integration Method}

Time dependent sub-cavity liquid volumes were obtained by numerically integrating the subcavity film thickness data at fixed times versus the cavity radius, based on the mapped cavity film thickness histories. Since the sub-cavity volume is the quantity of primary interest, the measured volumes when the droplet is initially forming the cavity, and after the cavity has filled back in, have not been calculated. The film thicknesses of these volumes have been judged to be too thick to have a susceptibility to onset of boiling or dry out similar to the sub-cavity volume. Sub-cavity volumes for specific times were computed utilizing the following integral, which has been evaluated using the trapezoidal rule

$$
\operatorname{Vol}_{i}=2 \pi \int_{0}^{R_{c_{i}}}(R * h) d R,
$$


where the $i$ subscript indicates the time index for which the volume is computed, and $R_{c_{i}}$ indicates the maximum sub-cavity radius at the current time.

\section{Section 5.3: Sub-cavity Volume Noise Removal}

Limitations of the CHR instrumentation required further post-processing of the sub-cavity thickness histories before the sub-cavity volumes can be computed. Thickness values that were beyond the sensor measuring range, and complex curvature in the free surface would cause data drop out, or noise. Sub-cavity thickness readings are generally very repeatable and reliable; with a majority of issues occurring with the first droplet impact, the collapse of crown walls, and the complex events during and after cavity fill in (e.g., impact of a secondary droplet into the cavity). By referring to the corresponding high-speed videos, any noise within the cavity lifetime was filtered out, and missing data points were then filled in with cubic interpolation. Generally, the greatest amount of noise was observed at the outer most radii (usually the last 1 - 3 histories), where it was more prominent for the thicker initial films. Typically very little filtering was required for the remaining inner radii. Initial film thicknesses of $h_{0}{ }^{*}<0.5$ resulted in the clearest histories since the initial liquid layer thickness was well below the CHR sensor measuring range. This allowed more film thicknesses greater than $h_{0}{ }^{*}$ to be captured without data drop out.

Figure 5.1 shows an example of the interpolated and filtered sub-cavity history overlaid with the raw data at the outer most radial location $\left(R_{c}=6.41 \mathrm{~mm}\right)$ measurement for an initial single drop condition of $W e=621, \operatorname{Re}=2,640, F r=415$, and $h_{0}{ }^{*}=0.5$. Nearly every history for this entire data set did not require any data removal in the minimum sub-cavity film thickness region $(0.014$ to 0.028 seconds for Figure 5.1). The cavity formation and receding phase shown in Figure 5.1, times of 0.0023 to 0.014 seconds and 0.028 to 0.034 seconds respectively, needed to be manually filtered. Generally, any data forming consistent trend patches such as the data from 0.034 to 0.038 seconds in 
Figure 5.1 were retained. Sparsely spaced isolated singular data points usually forming spikes were removed unless they formed part of an obvious greater trend or had clear video evidence of their correctness. For the example shown in Figure 5.1 data were removed between $0.004 \leq t \leq 0.01$ seconds and $0.029 \leq t \leq 0.033$ seconds. The removal of the lower portion of data $h<1000 \mu \mathrm{m}$ between $0.004 \leq t \leq 0.01$ was justified since the corresponding video showed smooth cavity propagation, so this noise was determined to be a product of the cavity curvature. The data consisting of the upper portion $(h>1000 \mu \mathrm{m})$ was believed to be a result of the sensor attempting to read the propagating crown that should be beyond its measuring range. This data could not be trusted so it was removed in the example. Similar logic was applied to the removed data between $0.029 \leq t \leq$ 0.033 seconds. In addition, no video evidence was observed of a contributing fluid structure for the larger thicknesses for this time frame. Similar noise behavior was consistent across the entire data set, so the same logic was applied to all cases.

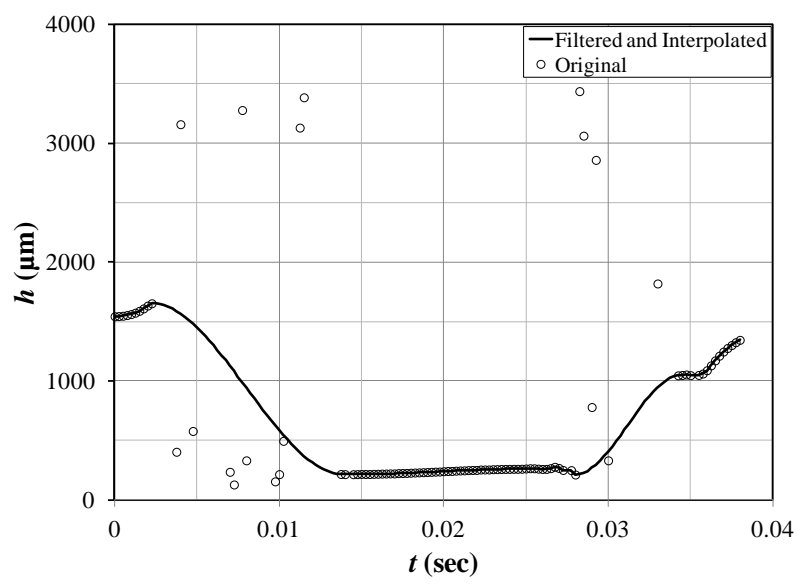

Figure 5.1: Filtered and interpolated sub-cavity film thickness history compared with the original $\left(W e=621, R e=2,640, F r=415, h_{0}{ }^{*}=0.5\right.$, and $\left.R_{c}=6.41 \mathrm{~mm}\right)$.

\section{Section 5.4: Sub-cavity Volume Radial Film Thickness Profile Preprocessing}

Sub-cavity liquid film thickness values at intermediate radii were linearly interpolated for each time step to increase the resolution of the volume calculations. The filtered and interpolated 
cavity film thickness histories were utilized in the radial interpolation. This typically resulted in an additional 95 histories between each measured radial location. An example surface contour plot of the final interpolated sub-cavity film thickness plotted versus time and radius is shown in Figure 5.2.

\section{Section 5.5: Sub-cavity Volume Iterative Technique}

The desired sub-cavity liquid volume was computed as the integral of the measured $h(R)$ at each time, as in Equation 5.1. The desired volume consisted of the "floor" of the example surface contour plot shown in Figure 5.2 b), basically in the dark blue region of the plot (roughly $120<h<$ $160 \mu \mathrm{m})$. The sub-cavity thickness surface plots such as the sample in Figure $5.2 \mathrm{~b}$ ) have been constructed from assembling each of the individual film thickness histories at different radii for the same droplet impact conditions.

Two issues arose in determining the sub-cavity volume: first, at what time to begin computing these volumes, and second, the choice of the radial location at which to end the integration at each time. The difficulty was generally due to a film thickness fillet versus time (referred to herein as a time fillet) as the cavity propagates to the impact surface and transitions into a (nearly) uniform film thickness after droplet impact. There is also a spatial fillet of thickness variation as the film transitions into the crown wall at each time, referred herein as the cavity fillet. Figure 5.2 a) shows an example of the time fillet and in Figure 5.2 b) the cavity fillets are observable at the outer radial locations. Determining how much of the spatial fillets to include in the volume calculation proved challenging as most of the fillet is too thick and has too short of a lifetime to be expected to contribute to the cavity dry out. As of this time, a universal set of conditions to determine this transition have not been developed. 


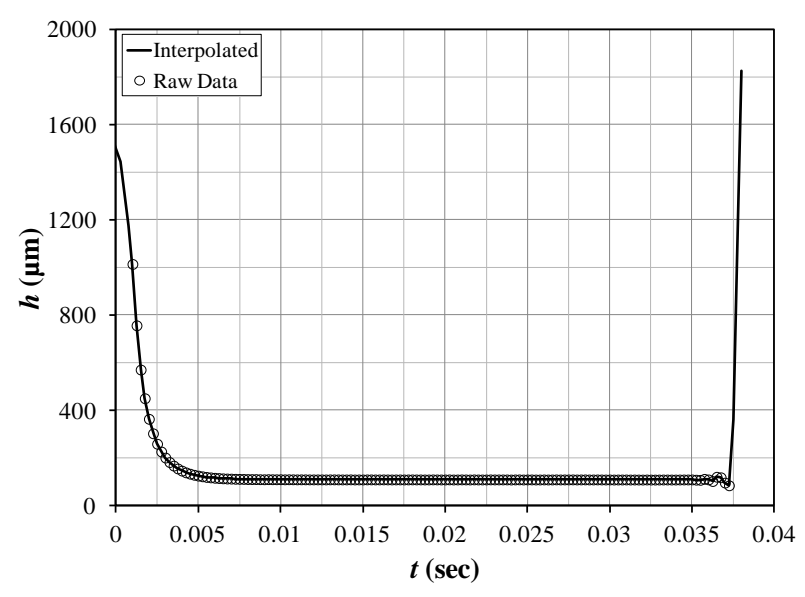

a)

Figure 5.2: Interpolated and filtered sub-cavity film thickness vs. time and mapped cavity (We $=621, \operatorname{Re}=2,640, F r=415$, and $\left.h_{0}{ }^{*}=0.5\right)$. a) Centerline thickness history. b) Sub-cavity thickness surface plot (Hillen et al. 2013).

Rather than use an arbitrary set of conditions to define the beginning of the sub-cavity liquid film region and the maximum radial integration limit, the following iterative process was developed. Volumes defined using Equation 5.1 were integrated radially outward using the trapezoidal rule. More specifically, at each time of interest the integration stops at several different radial locations corresponding to a film thickness beyond a selected film thickness constraint limit. The initial film thickness constraint limits were chosen as the average constant minimum film thickness on the drop impact centerline and 2.5 times this value, along with three equally spaced intermediate values. The minimum film thickness constraint value proved inappropriate since this value would fail to capture a significant portion of the sub-cavity volume of interest while twice its value resulted in exaggerated results. The lowest constraint limit was increased until sub-cavity volume curves with minimal noise were produced that had realistic trends consistent with those computed with the upper limit. The upper constraint limit was then decreased until peaks due to steep slopes in the time fillets shown Figure 5.2 a) at the beginning of the upper limit iteration were damped out. The upper constraint was further refined such that the differences in the volume trends began to converge at the upper bounds. The percent variation between sub-cavity volumes for the final film thickness 
constraints in Figure 5.3 b) range from $0.61 \%$ to $1.26 \%$ between $12 \leq t \leq 28 \mathrm{msec}$, with an overall average variance of $0.92 \%$. Generally, the final upper and lower constraints were within $1.2-1.4$ times the averaged minimum film thickness along the droplet impact centerline. This process is summarized visually in Figures 5.4 a) and b), respectively, for the example sub-cavity volumes shown in Figures 5.3 a) and b), using the initial and final film thickness constraint results shown in Figures 5.2 a) and b).

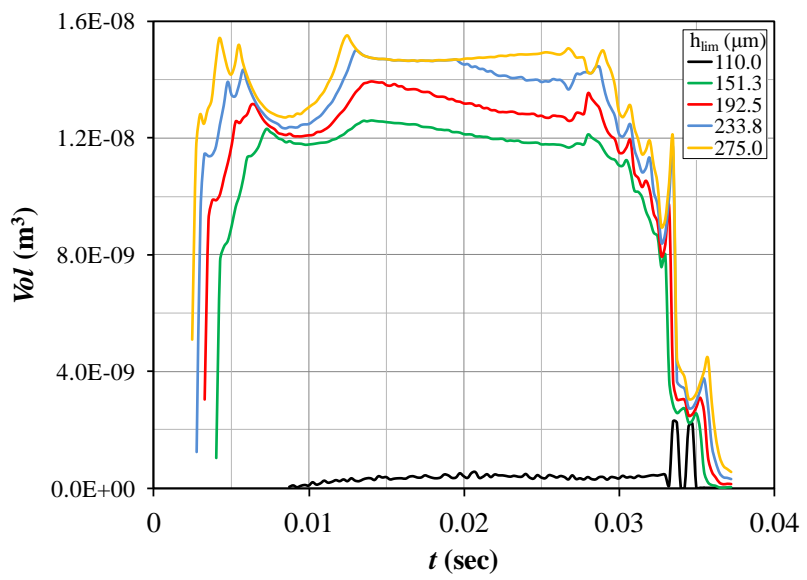

a)

Figure 5.3: Example of the iterative process of the sub-cavity volume trends vs. time ( $W e=621$, $R e=2,640, F r=415$, and $\left.h_{0}{ }^{*}=0.5\right)$. a) Initial constraints of the averaged and 2.5 times the minimum $h$. b) Final constraints (approximately 1.2 - 1.4 times the minimum $h$ ) (Hillen et al. 2013).

Further refinement and confirmation of realistic constraints were obtained by comparing the constraint limits with the corresponding droplet impact centerline history such as in Figure 5.2 a) and surface plots that are cropped using the film thickness constraint limits used to determine the radial limit. Figures 5.4 a) and b) shows cavity surface contour plots cropped and rescaled to the respective initial lower and upper constraint limits. Consistent with the sub-cavity volume trends in Figure 5.3 a), the constraint limits used in Figures 5.4 a) and b) significantly underestimate and overestimate the sub-cavity volume. In Figure 5.4 a) the minimum film thickness constraint limit caused the integration to end at nominally $R_{c}=2 \mathrm{~mm}$ neglecting most of the cavity region of interest. Too much 
of the time and cavity fillets were included for the initial upper constraint of 2.5 times the minimum film thickness as depicted in Figure 5.4 b). Figures 5.4 c) and d) are the corresponding cropped and rescaled cavity profiles for the final constraint limits, displaying a realistic representation of the subcavity liquid film thickness profile versus radius and time. The sub-cavity volumes computed with the middle film thickness constraint obtained at the end of the iterative process have been selected as the best representation for the final sub-cavity volume results presented in Section 7.8.

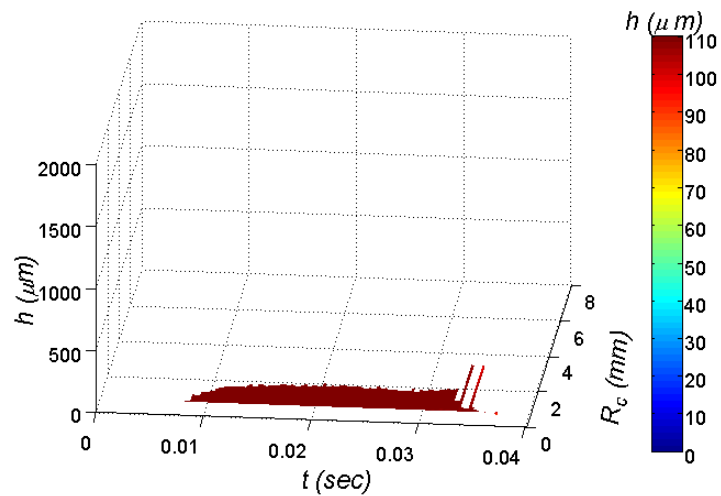

a)

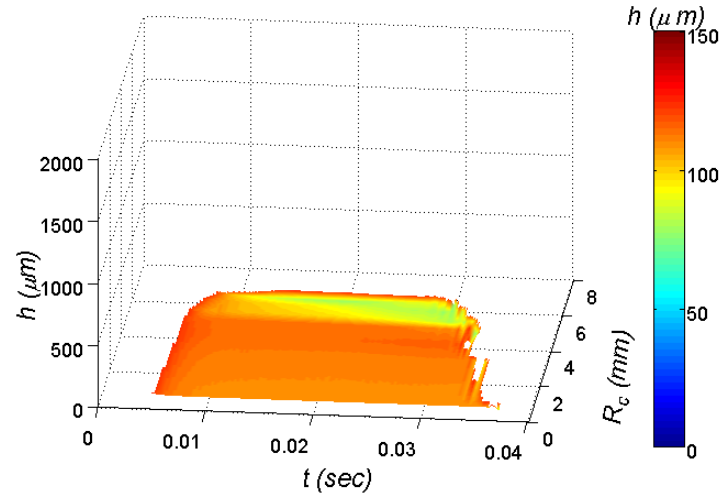

c)

Figure 5.4: Cropped cavity surface plots $\left(W e=621, \operatorname{Re}=2,640, \mathrm{Fr}=415\right.$, and $\left.h_{0}{ }^{*}=0.5\right)$. a) Initial lower limit constraint. b) Initial upper limit constraint. c) Final iterated lower limit constraint. d) Final iterated upper limit constraint (Hillen et al. 2013).

\section{Section 5.6: Sub-cavity Volume Accuracy Check}

A check of the accuracy of the adopted sub-cavity volume calculation process was obtained through the following procedure. The computed weighted average of the sub-cavity minimum film 
thickness, versus time and radius, found as depicted for the example discussed in Figures 5.1 - 5.4, is shown in Figure 5.5.

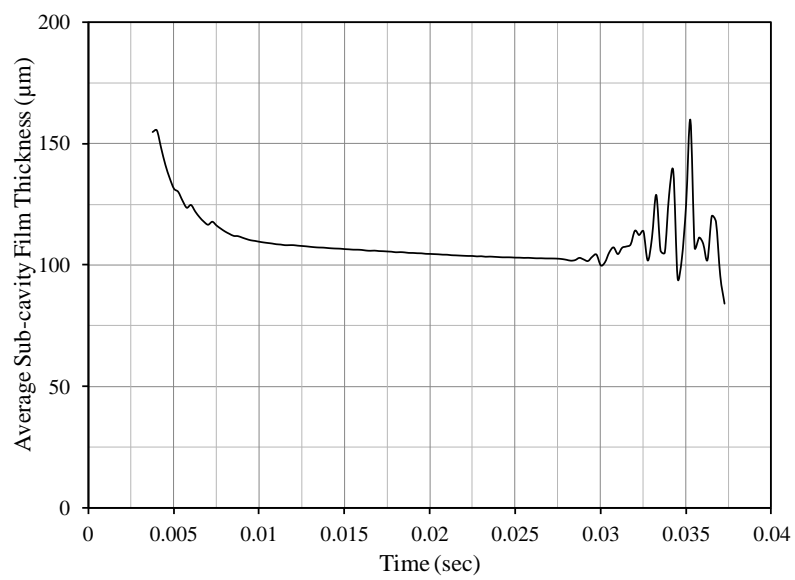

Figure 5.5: Estimated global minimum sub-cavity film thickness versus time.

The averaged minimum film thickness was multiplied by the cavity total area versus time which was computed using the measured sub-cavity radius versus time. Figure 5.6 shows that volumes computed using the sub-cavity radii from the SV and BV were on the same order of magnitude as the volumes computed by the main process. In fact, sub-cavity volume estimates using the cavity radii obtained by the BV method gave reasonably good agreement with the sub-cavity volumes computed by numerical integration and the main iterative method for this one case that was explained.

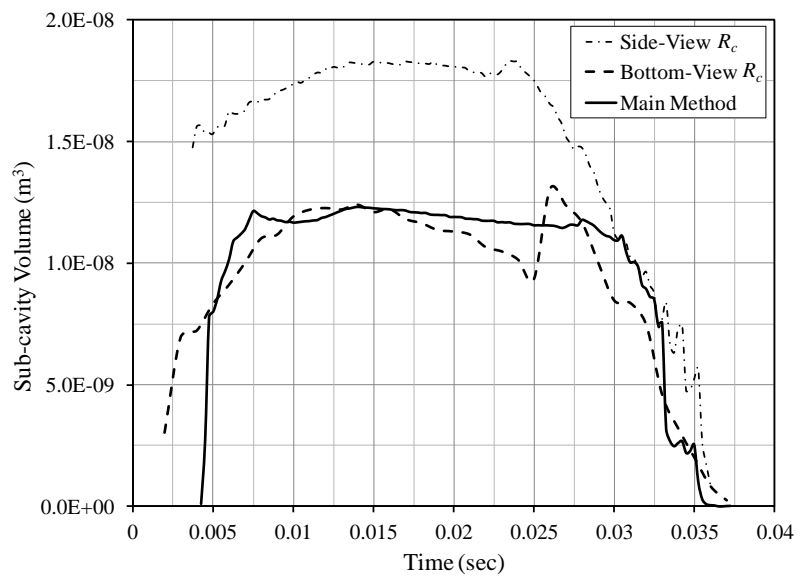

Figure 5.6: Sub-cavity volumes computed from the optimized numerical integration method compared with estimates using the averaged $h_{\min }$ and $\boldsymbol{R}_{c}$ (Hillen et al., 2013). 


\section{CHAPTER 6: $\quad$ SPRAY RESULTS AND DISCUSSION}

\section{Section 6.1: Introduction}

Results presented in chapter consist of the characterization of the spray of interest via PDPA, both with and without the impact surface in place. An initial form of these results has been presented in Hillen and Kuhlman (2013a). The spray droplet diameter and velocity results without the impact surface installed have been utilized in creating the single droplet sub-cavity film thickness experimental test matrix.

Spray droplet size and velocity trends and effects of varying the nozzle flow conditions without the impact surface installed are reported in Section 6.2. Also, the effects of varying the standoff measuring distance from the nozzle are examined. The section is concluded with the nozzle of interest characterized in terms of a single set of dimensionless Weber, Reynolds, and Froude numbers at each nozzle pressure.

Data for the characterization of the splashed spray droplets generated by the spray impinging onto the impact surface are documented in Section 6.3. The incoming spray droplet data with the impact surface installed have been compared with the corresponding basic spray results (spray measurements without the impact surface installed). The trends and effects of varying the flow conditions on the splashed droplets are discussed in this section. Splashed droplet data measured traversing vertically to the side of the impact surface are reported for comparison with the splashed data collected for radial traverses above the impact surface. Weber, Reynolds, and Froude numbers were also computed for the integrated global averages of the splashed droplets to investigate if the splashed droplets contribute to cavity formation.

The limitations of PDPA to measure volume fluxes are briefly discussed in Section 0.0. The development of the detailed test matrix for relevant spray conditions is then detailed in Section 6.5. 


\section{Section 6.2: Spray Results and Discussion: Without Spray Impact Surface Installed}

The data presented in this section focuses on the droplet diameter and velocity distributions measured using PDPA in the spray of interest without the impact surface installed. Figure 6.1 presents typical sample histograms of the spray data for the highest pressure case (4.14 bar gage pressure) listed in Table 3.3 at two measuring locations: at the spray center $\left(R_{s}=0 \mathrm{~cm}\right)$, and at the spray edge $\left(R_{s}=2.47 \mathrm{~cm}\right)$ for a standoff distance of $z=3.81 \mathrm{~cm}$. Data rates were much larger towards the center of the spray region due to a higher flux of droplets. Also, droplet sizes were noticeably smaller at the centerline than the edge of the spray, and a double peak in the droplet diameters can be noticed in Figure 6.1 a). Further comparison of Figures 6.1 a) and b) shows that the two locations share similar overall droplet velocity magnitudes and trends. However the droplets at $R_{s}=0 \mathrm{~cm}$ in Figure 6.1 a) have much larger axial velocities and a nearly negligible average radial velocity component compared to droplets at $R_{s}=2.47 \mathrm{~cm}$ in Figure $6.1 \mathrm{~b}$ ) which have relatively high radial velocities, but smaller axial velocities. This is reasonable since the droplets at the center of the spray should be moving virtually normal to the nozzle. Also, the droplet radial velocity should increase with the impact angle away from the centerline. Additional sample histograms similar to those in Figure 6.1 for $R_{s}=0 \mathrm{~cm}$ and $R_{s}=2.47 \mathrm{~cm}$ at $z=3.81 \mathrm{~cm}$ for the two lower pressure cases in Table 3.3 are provided in Appendix B. 

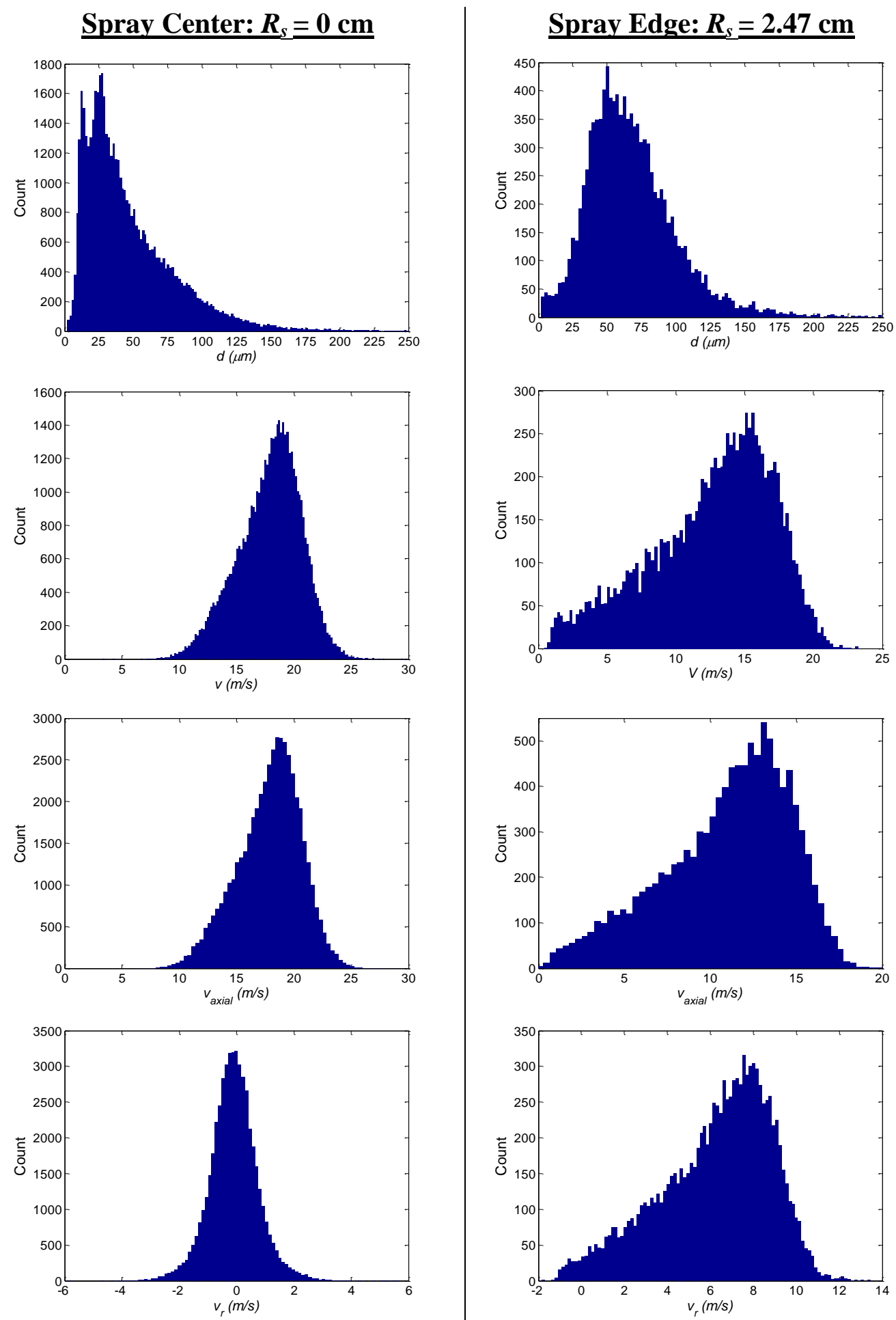

a)

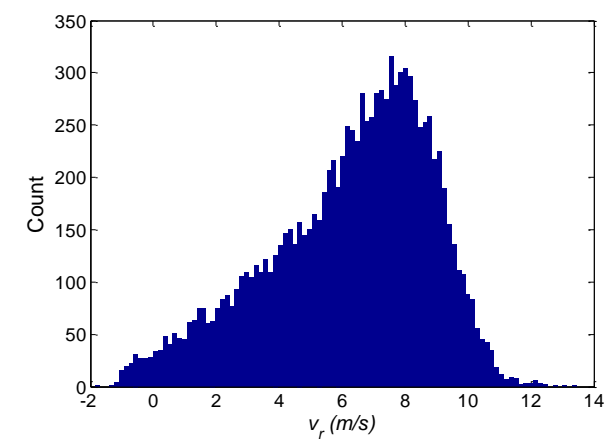

b)

Figure 6.1: Spray droplet diameter, speed, axial velocity, and radial velocity for a 4.14 bar and $54.5 \mathrm{~L} / \mathrm{hr}$ at $z=3.81 \mathrm{~cm}$. a) Center of the spray, $R_{s}=0 \mathrm{~cm}$. b) Edge of spray, $R_{s}=2.47 \mathrm{~cm}$. 


\section{Section 6.2.1: Spray Trends and Effects of Varying the Nozzle Flow Condition}

The effects of varying the spray pressure and flow rate on the arithmetic and Sauter mean diameters at $z=3.81 \mathrm{~cm}$ are shown in Figure $6.2 \mathrm{a}$ ) and b) respectively. It was observed that increases in pressure and flow rate generated smaller droplets, which was consistent with similar results presented by Kalantari and Tropea (2007), and Winkler and Peters (2002). Consistent with Figure 6.1, the arithmetic mean droplet sizes in Figure 6.2 a) decrease near the spray centerline where the spray is the most atomized. Centerline arithmetic mean diameters in Figure 6.2 a) are nominally $47 \mu \mathrm{m}, 54 \mu \mathrm{m}$, and $74 \mu \mathrm{m}$ for the respective 4.14 bar, 2.76 bar, 1.38 bar spray gage pressures. The studied spray produced nearly constant Sauter mean diameters of nominally $110 \mu \mathrm{m}$, $130 \mu \mathrm{m}$, and $170 \mu \mathrm{m}$ for the respective $1.38 \mathrm{bar}, 2.76 \mathrm{bar}$, and 4.14 bar spray cases across the span of the spray as shown in Figure $6.2 \mathrm{~b}$ ). Evidence that the spray cone was not fully formed for the 1.38 bar, $29.5 \mathrm{~L} / \mathrm{hr}$ spray can be observed in the variations in the arithmetic mean diameters past $R_{S}= \pm 20$ $\mathrm{mm}$ in Figure $6.2 \mathrm{a}$ ). Good agreement is observed between the $x$ and $y$-axis profiles for both the arithmetic and Sauter mean diameters. Uncertainty bars based on 95\% confidence interval shown in Figure 6.2 are presented at the spray center $\left(R_{s}=0 \mathrm{~cm}\right)$, edge $\left(R_{s}=2.68 \mathrm{~cm}\right)$, and half way in between $\left(R_{S}=1.34 \mathrm{~cm}\right)$. 


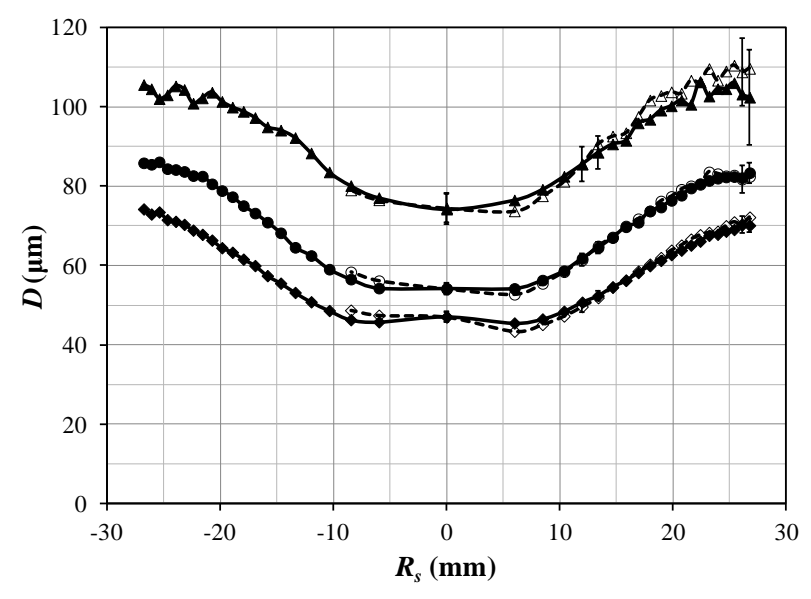

a)

Figure 6.2: Droplet diameter profiles at a constant $z=3.81 \mathrm{~cm}$ for the different pressure cases with uncertainty bars. a) Arithmetic mean diameter. b) Sauter mean diameter. $\Delta \triangle 1.38$ bar, 29.5 L/hr; $\bigcirc 2.76$ bar, $43.5 \mathrm{~L} / \mathrm{hr} ; \diamond \diamond 4.14$ bar, $54.5 \mathrm{~L} / \mathrm{hr}$; — $x$-plane data; ---- $y$-plane (Hillen et al., 2013).

The global average difference of the $D$ profiles between different pressure settings are decreases of $22.9 \mu \mathrm{m}, 12.4 \mu \mathrm{m}$, and $35.3 \mu \mathrm{m}$ between the respective flow conditions of $1.38-2.76$ bar, 2.76 - 4.14 bar, and 1.38 - 4.14 bar. The corresponding differences for the $D_{32}$ profiles between different pressure settings are $35.9 \mu \mathrm{m}, 20.4 \mu \mathrm{m}$, and $56.4 \mu \mathrm{m}$ between the flow conditions of 1.38 2.76 bar, 2.76-4.14 bar, and 1.38 - 4.14 bar, respectively.

Figure 6.3 shows the effects of the nozzle pressure and flow rate on the average droplet velocity component profiles. It was observed that increasing the nozzle flow rate and pressure resulted in higher droplet speeds and greater droplet incidence angles towards the cone edge. Consistent with Figure 6.1 the average axial velocity increases and the radial velocity decreases to virtually zero at $R_{s}=0 \mathrm{~mm}$ when approaching the spray centerline. Maximum axial velocities from Figure 6.3 a) were measured to be nominally $9.8 \mathrm{~m} / \mathrm{s}, 14 \mathrm{~m} / \mathrm{s}$, and $18 \mathrm{~m} / \mathrm{s}$ for the respective flow conditions of 1.38 bar, 2.76 bar, 4.14 bar. The largest radial velocities measured in Figure 6.3 b) are nominally $4.1 \mathrm{~m} / \mathrm{s}, 5.5 \mathrm{~m} / \mathrm{s}, 6.4 \mathrm{~m} / \mathrm{s}$ for the respective flow conditions of 1.38 bar, 2.76 bar, and 4.14 bar. Measurements showed negligible swirl velocity as was expected for this non-swirl nozzle. The 
radial velocity component in Figure 6.3 b) could only be measured along the $x$-axis of the spray, since the PDPA measured the swirl velocity component for the $y$-axis traverse. The average axial velocities that have been recorded along the y-axis exhibit some discrepancy with the measurements along $x$-axis over most of the spray. It is believed that this is due to the PDPA measuring volume length being much longer than its diameter as listed in Table 3.1, resulting in the $y$-axis profile being less sensitive to the density of the spray. Uncertainty bars shown in Figure 6.3 are presented at the spray center $\left(R_{s}=0 \mathrm{~cm}\right)$, edge $\left(R_{s}=2.68 \mathrm{~cm}\right)$, and half way in between $\left(R_{s}=1.34 \mathrm{~cm}\right)$. The methodology used in determining the uncertainty bars in Figures 6.2 and 6.3 will be explained in Section 6.2.2.

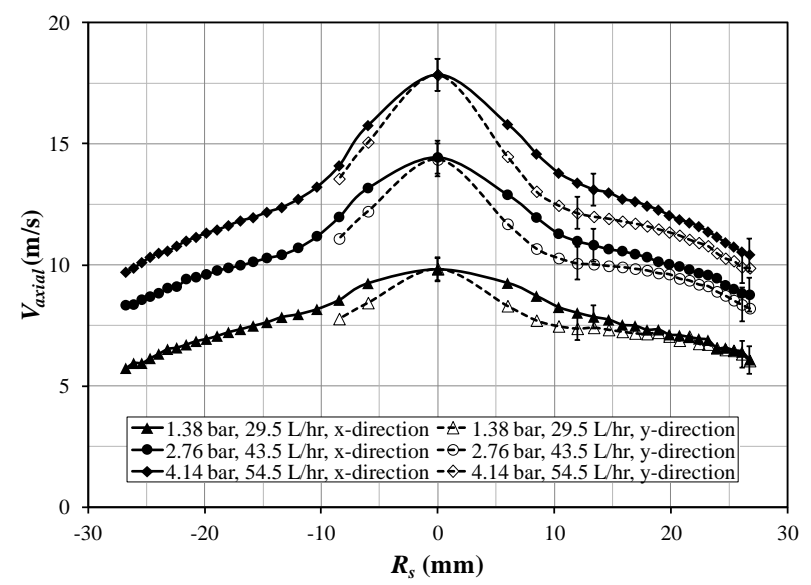

a)

Figure 6.3: Spray flow condition effects on the mean spray velocity components at $z=3.81 \mathrm{~cm}$ with uncertainty bars. a) Mean axial velocity profile. b) Mean radial velocity profile (Hillen $e t$ al., 2013).

The global average difference of the $V_{\text {axial }}$ profiles between the different pressure settings are increases of $2.96 \mathrm{~m} / \mathrm{s}, 2.07 \mathrm{~m} / \mathrm{s}$, and $5.02 \mathrm{~m} / \mathrm{s}$ for the respective flow conditions of $1.38-2.76$ bar, 2.76 - 4.14 bar, and 1.38 - 4.14 bar. The corresponding differences for the $V_{r}$ profiles between pressure settings are increases of $0.906 \mathrm{~m} / \mathrm{s}, 0.597 \mathrm{~m} / \mathrm{s}$, and $1.50 \mathrm{~m} / \mathrm{s}$ for the respective flow conditions of 1.38 - 2.76 bar, 2.76 - 4.14 bar, and 1.38 - 4.14 bar, respectively. 
Appendix B contains the graphs similar to Figures 6.2 and 6.3, but for data collected at standoff distances of $z=3.18$ and $4.45 \mathrm{~cm}$ in addition to the velocity magnitude for all $z$ profile locations. The spray profile graphs shown in Appendix B followed identical trends observed in Figures 6.2 and 6.3 when the spray pressure and flow rate were varied.

\section{Section 6.2.2: Uncertainty Analysis of PDPA Spray Data (No Target Surface)}

An uncertainty analysis of the PDPA spray results has been performed for the data presented in Section 6.2.1. There are three main contributing factors to the uncertainty in the PDPA measurements for the $D, D_{32}, V_{\text {axial }}$, and $V_{r}$ : the $0.75 \%$ instrumentation uncertainty reported by the manufacturer, the statistical fluctuation of the data for each measurement location, and the uncertainty between the $x$ and $y$-traverse profiles due to the spray density effects on the PDPA probe volume (referred to as the PDPA probe volume uncertainty). The uncertainty for $V_{r}$ consists of only the statistical fluctuation and instrumentation uncertainty since the $y$-profile is the swirl velocity which is $0 \mathrm{~m} / \mathrm{s}$, so there is no way to quantify this effect on $V_{r}$.

As mentioned in Section 6.2.1 there is difference in the measurements between the $x$ and $y$ axis profiles even though it was an axisymmetric spray. This difference between the $x$ and $y$-axis profiles is the PDPA probe volume uncertainty. This uncertainty is due to data taken for the $x$ traverse experiencing more interference from the spray density as shown in Figure 6.4. The transmitting and receiving optics were located on the $+y$ portion of the spray. 


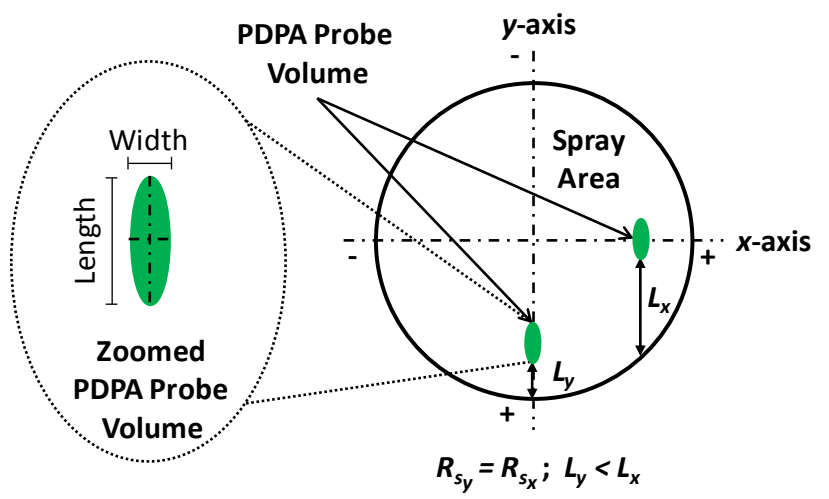

Figure 6.4: A sketch of the PDPA probe volume at equal $R_{s}$ locations on the $x$ and $y$-axis to demonstrate the increased interference of the $x$-traverse due to the spray density.

The PDPA probe volume uncertainty for each corresponding spatial location is computed by:

$$
\Delta(\Psi)_{P V}=\left|\Psi_{x}\left(R_{S}\right)-\Psi_{y}\left(R_{S}\right)\right|
$$

The symbol $\Psi_{x \text { or } y}\left(R_{s}\right)$ represents the average value of $D, D_{32}, V_{\text {axial }}$, or $V_{r}$ at a distance from the spray axis. The subscript $x$ denotes values measured along the $x$-traverse. Because the $x$-traverse extended across the spray axis, values were computed according to $\Psi_{x}\left(R_{s}\right)=1 / 2\left[\Psi\left(x=R_{s}\right)+\Psi(x=\right.$ $\left.R_{s}\right)$ ]. The subscript $y$ denotes values measured along the $y$-traverse, which did not fully extend across the axis. It should be noted that since the $y$-traverse measured the swirl velocity a PDPA probe volume uncertainty cannot be computed for the radial velocity component. Table 6.1 contains the global average, maximum, and minimum PDPA probe volume uncertainty between the $x$ and $y$-axis measurements for the arithmetic and Sauter mean diameters in Figure 6.2. There is very little variation between the two measuring profiles in Table 6.1 further supporting good agreement between the $x$ and $y$-axis measurements. The larger differences in $D$ for $P=1.38$ bar is due to the spray cone not being fully form resulting in data fluctuations towards the spray edges as shown in Figure 6.2 a). 
Table 6.1: Average, maximum, and minimum PDPA probe volume uncertainty between the $x$ and $y$-traverse profiles of the arithmetic and Sauter mean diameter for varying pressures at a constant $z=3.81 \mathrm{~mm}$.

\begin{tabular}{|c|c|c|c|c|c|c|}
\hline $\begin{array}{c}P \\
\text { (bar) }\end{array}$ & $\begin{array}{c}\overline{\Delta(\boldsymbol{D})_{P V}} \\
(\boldsymbol{\mu} \mathbf{m})\end{array}$ & $\begin{array}{c}\Delta(\boldsymbol{D})_{P V_{\max }} \\
(\boldsymbol{\mu m})\end{array}$ & $\begin{array}{c}\Delta(\boldsymbol{D})_{P V_{\text {min }}} \\
(\boldsymbol{\mu \mathrm { m }})\end{array}$ & $\begin{array}{c}\overline{\Delta\left(D_{32}\right)_{P V}} \\
(\mu \mathrm{m})\end{array}$ & $\begin{array}{c}\Delta\left(\boldsymbol{D}_{32}\right)_{P V_{\max }} \\
(\boldsymbol{\mu \mathrm { m } )})\end{array}$ & $\begin{array}{c}\Delta\left(\boldsymbol{D}_{32}\right)_{P V_{\text {min }}} \\
(\boldsymbol{\mu \mathrm { m } )})\end{array}$ \\
\hline 1.38 & 2.82 & 6.42 & 0.214 & 7.82 & 21.0 & 0.142 \\
\hline 2.76 & 0.767 & 2.20 & 0.0483 & 8.93 & 18.0 & 2.12 \\
\hline 4.14 & 0.703 & 2.22 & 0.00284 & 7.56 & 13.9 & 1.25 \\
\hline
\end{tabular}

Table 6.2 lists the global average, the maximum, and the minimum PDPA probe volume uncertainty for the axial velocity between the $x$ and $y$-axis measurements in Figure 6.3. The global average $V_{\text {axial }}$ PDPA probe volume uncertainty is lower since the equal area traversing method produces more data points towards the spray's edge where the data is in closer agreement. The maximum $V_{\text {axial }}$ uncertainty generally occurs between $0 \leq R_{s} \leq 1.8 \mathrm{~cm}$ where the measuring volume on the $x$-traverse has the largest interference from the spray density.

Table 6.2: Average, maximum, and minimum PDPA probe volume uncertainty between the $x$ and $y$-axis measurements of the mean axial velocity for varying pressures at a constant $z=3.81$ mm.

\begin{tabular}{|c|c|c|c|}
\hline $\boldsymbol{P}($ bar $)$ & $\overline{\Delta\left(\boldsymbol{v}_{\text {axial }}\right)_{\boldsymbol{P V}}}(\mathrm{m} / \mathbf{s})$ & $\Delta\left(\boldsymbol{v}_{\text {axial }}\right)_{\boldsymbol{P V} \max }(\mathbf{m} / \mathbf{s})$ & $\Delta\left(\boldsymbol{v}_{\text {axial }}\right)_{\boldsymbol{P V}} \min$ \\
\hline 1.38 & 0.275 & 0.930 & 0.00686 \\
\hline 2.76 & 0.442 & 1.34 & 0.0863 \\
\hline 4.14 & 0.497 & 1.29 & 0.0120 \\
\hline
\end{tabular}

The statistical fluctuation uncertainty is based on the variation of the measured droplet characteristics, the total number of measurement samples, and its corresponding decorrelation sample index at a specified radial location. Equation 6.2 is an attempt to quantify this uncertainty for both the $x$ and $y$-axis measurements even though the PDPA samples are at random increments and not at equal time samples. The equation is rigorously valid for data taken with a constant time interval between samples for a stationary signal, but for PDPA the sample interval varies.

$$
\Delta\left(\psi\left(R_{s}\right)\right)_{\text {stat }}=\frac{s t d\left(\psi\left(R_{s}\right)\right)}{\sqrt{\frac{N}{n_{d e c}}}}
$$


The $\psi\left(R_{s}\right)$ represents the individual values of $d, v_{\text {axial }}$, and $v_{r}$ at a distance $R_{s}$ from the spray axis. Statistical fluctuation uncertainties were computed for the $x$ and $y$-axis measurements at radial locations of $0,1.34$, and $2.68 \mathrm{~cm}$. These were not calculated for the radial velocity for the $y$-axis measurements since it is the swirl velocity. The total number of measured samples $(N)$ used in Equation 6.2 is based on either the PDPA system's time out (30 sec), or total sample limit $(50,000)$ listed in Table 3.1. The decorrelation sample index in Equation 6.2 was computed from the indexed total measurement sample to ensure the correct value. Figure 6.5 is example of the axial velocity correlation coefficient versus the sample index for a flow condition of 4.14 bar and $54.5 \mathrm{~L} / \mathrm{hr}$ at $R_{s}=$ $0 \mathrm{~cm}$ and $z=3.81 \mathrm{~cm}$, where the decorrelation sample index was taken as the separation between the sequential data sample where the correlation coefficient decays to zero.

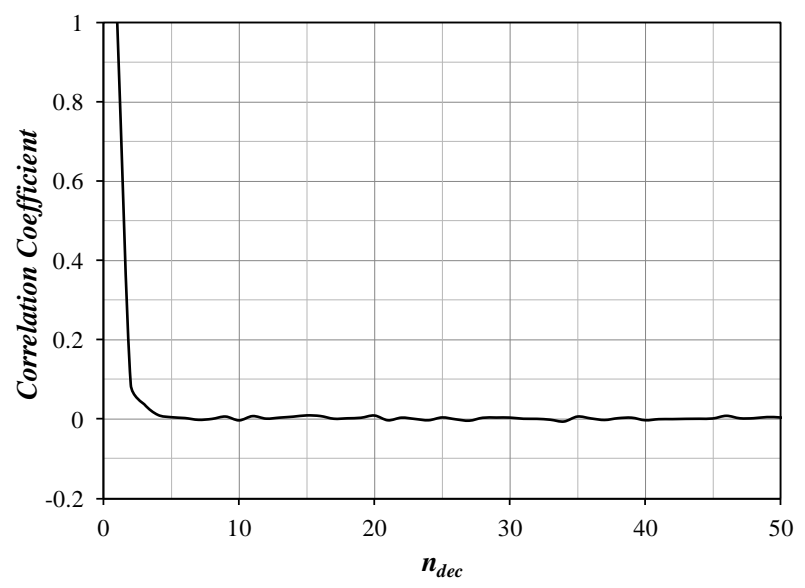

Figure 6.5: Example of the correlation coefficient versus the sample index for the axial velocity for a flow condition of $4.14 \mathrm{bar}$ and $54.5 \mathrm{~L} / \mathrm{hr}$ at $R_{s}=0 \mathrm{~cm}$ and $z=3.81 \mathrm{~cm}$.

Table 6.3 contains the total measurement sample size and decorrelation sample index values for the measurements located at $R_{s}=0,1.34,2.68 \mathrm{~cm}$ for the three pressure cases. Typically the total measurement samples are similar between the $x$ and $y$-traverses except at $R_{s}=1.34 \mathrm{~cm}$ where the $x$ axis measurements have nominally 2,000 - 5,000 more samples. The decorrelation sample index values are all generally small for the droplet characteristics, ranging in value from 3 to 12 . 
Table 6.3: The total measurement sample and the decorrelation sample index of the droplet diameter, axial velocity, and radial velocity at $z=3.81 \mathrm{~cm}$ for the $x$ and $y$-traverses $\left(\right.$ no $n_{d e c}\left(v_{r}\right)$ for the $y$-traverse).

\begin{tabular}{|c|c|c|c|c|c|c|c|c|}
\hline $\boldsymbol{P}(\mathbf{b a r})$ & $\boldsymbol{R}_{\boldsymbol{s}}(\mathbf{c m})$ & $\boldsymbol{N}_{\boldsymbol{x}}$ & $\boldsymbol{n}_{\text {dec }}(\boldsymbol{d})_{\boldsymbol{x}}$ & $\boldsymbol{n}_{\text {dec }}\left(\boldsymbol{v}_{\text {axial }}\right)_{\boldsymbol{x}}$ & $\boldsymbol{n}_{\text {dec }}\left(\boldsymbol{v}_{\boldsymbol{r}}\right)_{\boldsymbol{x}}$ & $\boldsymbol{N}_{\boldsymbol{y}}$ & $\boldsymbol{n}_{\text {dec }}(\boldsymbol{d})_{\boldsymbol{y}}$ & $\boldsymbol{n}_{\text {dec }}\left(\boldsymbol{v}_{\text {axial }}\right)_{\boldsymbol{y}}$ \\
\hline \multirow{4}{*}{1.38} & 0 & 26,967 & 8 & 3 & 6 & 27,306 & 6 & 3 \\
\cline { 2 - 9 } & 1.34 & 8,590 & 4 & 3 & 4 & 6,503 & 4 & 3 \\
\cline { 2 - 9 } & 2.68 & 643 & 7 & 3 & 3 & 694 & 3 & 3 \\
\hline \multirow{4}{*}{2.76} & 0 & 50,000 & 4 & 8 & 5 & 50,000 & 3 & 8 \\
\cline { 2 - 9 } & 1.34 & 27,406 & 3 & 5 & 5 & 22,556 & 4 & 4 \\
\cline { 2 - 9 } & 2.68 & 3,092 & 2 & 3 & 2 & 2,893 & 5 & 4 \\
\hline \multirow{4}{*}{4.14} & 0 & 50,000 & 5 & 7 & 5 & 50,000 & 4 & 12 \\
\cline { 2 - 9 } & 1.34 & 48,364 & 3 & 7 & 12 & 44,611 & 4 & 5 \\
\cline { 2 - 9 } & 2.68 & 6,923 & 2 & 5 & 5 & 6,257 & 4 & 3 \\
\hline
\end{tabular}

The standard deviation in the measurements of the $d, v_{a x i a l}$, and $v_{r}$ used in Equation 6.2 are listed in Table 6.4. The deviation in the data is similar between the corresponding $x$ and $y$-axis profiles with the largest deviation in the velocities occurring at $R_{s}=1.34 \mathrm{~cm}$. The deviation for the droplet diameters decreased and the deviation of the velocity components increased with increasing the pressure.

Table 6.4: The standard deviation of the droplet diameter, axial velocity, and radial velocity at $z=3.81 \mathrm{~cm}$ for the $x$ and $y$-traverses (no $\operatorname{std}\left(v_{r}\right)$ for the $y$-traverse).

\begin{tabular}{|c|c|c|c|c|c|c|}
\hline $\begin{array}{c}P \\
(\mathbf{b a r})\end{array}$ & $\begin{array}{c}\boldsymbol{R}_{\boldsymbol{s}} \\
(\mathbf{c m})\end{array}$ & $\begin{array}{c}\text { std }(\boldsymbol{d})_{\boldsymbol{x}} \\
(\mathbf{u m})\end{array}$ & $\begin{array}{c}\text { std }\left(\boldsymbol{v}_{\text {axial }}\right)_{\boldsymbol{x}} \\
(\mathbf{m} / \mathbf{s})\end{array}$ & $\begin{array}{c}\boldsymbol{s t d}\left(\boldsymbol{v}_{\boldsymbol{r}}\right)_{\boldsymbol{x}} \\
(\mathbf{m} / \mathbf{s})\end{array}$ & $\begin{array}{c}\text { std }(\boldsymbol{d})_{\boldsymbol{y}} \\
(\mathbf{u m})\end{array}$ & $\begin{array}{c}\text { std }\left(\boldsymbol{v}_{\text {axial }}\right)_{\boldsymbol{y}} \\
(\mathbf{m} / \mathbf{s})\end{array}$ \\
\hline \multirow{4}{*}{1.38} & 0 & 57.9 & 1.69 & 0.629 & 59.0 & 1.75 \\
\cline { 2 - 7 } & 1.34 & 56.2 & 2.80 & 1.15 & 56.3 & 2.61 \\
\cline { 2 - 7 } & 2.68 & 55.4 & 2.46 & 1.76 & 59.6 & 2.23 \\
\hline \multirow{4}{*}{4.76} & 0 & 42.0 & 2.30 & 0.729 & 41.8 & 2.31 \\
\cline { 2 - 7 } & 1.34 & 40.2 & 3.65 & 1.56 & 39.2 & 3.56 \\
\cline { 2 - 7 } & 2.68 & 43.1 & 3.17 & 2.29 & 40.1 & 2.89 \\
\hline \multirow{3}{*}{4.14} & 0 & 35.7 & 2.78 & 0.843 & 36.4 & 2.76 \\
\cline { 2 - 7 } & 1.34 & 32.9 & 4.32 & 1.86 & 29.8 & 4.29 \\
\cline { 2 - 7 } & 2.68 & 34.2 & 3.55 & 2.63 & 34.2 & 3.45 \\
\hline
\end{tabular}

The final statistical fluctuation uncertainty of the droplet characteristics for the $x$ and $y$-axis profiles for the varying pressure and radial locations are presented in Table 6.5. These uncertainties 
have been computed with the values presented in Tables 6.4 and 6.3 using Equation 6.2. The most notable statistical fluctuation in the data is for the droplet diameter at the edge of the spray at $P=$ 1.38 bar for both traverse profiles. This, is again, due to the spray cone not being fully formed and atomized at this flow condition. The uncertainty of the $x$ and $y$-axis measurements are similar.

Table 6.5: The statistical fluctuation uncertainty of the droplet diameter, axial velocity, and radial velocity at $z=3.81 \mathrm{~cm}$ for the $x$ and $y$-traverse profiles (no $\Delta\left(v_{r}\right)_{\text {stat }}$ for the $y$-traverse).

\begin{tabular}{|c|c|c|c|c|c|c|}
\hline $\begin{array}{c}P \\
\text { (bar) }\end{array}$ & $\begin{array}{c}\boldsymbol{R}_{s} \\
(\mathbf{c m})\end{array}$ & $\begin{array}{c}\Delta(d)_{\text {stat }_{x}} \\
(\mathbf{u m})\end{array}$ & $\begin{array}{c}\Delta\left(v_{\text {axial }}\right)_{\text {stat }} \\
(\mathbf{m} / \mathbf{s})\end{array}$ & $\begin{array}{c}\Delta\left(v_{r}\right)_{\text {stat }_{x}} \\
(\mathbf{m} / \mathbf{s})\end{array}$ & $\begin{array}{c}\Delta(d)_{\text {stat }} \\
(\mathbf{u m})\end{array}$ & $\begin{array}{c}\Delta\left(v_{\text {axial }}\right)_{\text {stat }} \\
(\mathbf{m} / \mathbf{s})\end{array}$ \\
\hline \multirow{3}{*}{1.38} & 0 & 0.998 & 0.0178 & 0.00939 & 0.874 & 0.0183 \\
\hline & 1.34 & 1.21 & 0.0523 & 0.0247 & 1.40 & 0.0561 \\
\hline & 2.68 & 5.78 & 0.168 & 0.120 & 3.918 & 0.146 \\
\hline \multirow{3}{*}{2.76} & 0 & 0.376 & 0.0291 & 0.00729 & 0.324 & 0.0292 \\
\hline & 1.34 & 0.421 & 0.0493 & 0.0210 & 0.522 & 0.0474 \\
\hline & 2.68 & 1.10 & 0.0986 & 0.0583 & 1.67 & 0.108 \\
\hline \multirow{3}{*}{4.14} & 0 & 0.357 & 0.0329 & 0.00843 & 0.325 & 0.0427 \\
\hline & 1.34 & 0.259 & 0.0519 & 0.0292 & 0.282 & 0.0454 \\
\hline & 2.68 & 0.581 & 0.0954 & 0.0706 & 0.866 & 0.0755 \\
\hline
\end{tabular}

The total uncertainty for the PDPA spray data for $D$ and $V_{\text {axial }}$ in Section 6.2.1 is computed through the following equation:

$$
\Delta\left(\Psi\left(R_{S}\right)\right)_{\text {total }}=\sqrt{\left(2 * \Delta\left(\psi\left(R_{S}\right)_{\text {stat }}\right)^{2}+\left[\frac{1}{2} \Delta(\Psi)_{P V_{\text {max }}}\right]^{2}+\Delta\left(\Psi\left(R_{S}\right)\right)_{0.75 \%}^{2}\right.}
$$

The symbol $\Delta\left(\Psi\left(R_{s}\right)\right)$ represents an overall uncertainty estimate for $D, D_{32}, V_{a x i a l}$, or $V_{r}$ at a distance $R_{s}$ from the spray axis. The subscripts stat, $P V$, and $0.75 \%$ represent the respective statistical fluctuation uncertainty, the PDPA probe volume uncertainty, and instrumentation uncertainty. Half of the maximum $\Delta(\Psi)_{P V}$ from Tables 6.1 and 6.2 was determined to give the best representation of the PDPA probe volume uncertainty between the $x$ and $y$-traverse results. As previously mentioned PDPA probe volume uncertainties were not attainable for the radial velocity, thus it has been excluded in Equation 6.3 for $\Delta\left(V_{r}\left(\mathrm{R}_{s}\right)\right)_{\text {total. }}$. The contributions of the statistical fluctuation to the total uncertainty are generally very small. The dominant uncertainty is the maximum PDPA probe volume 
uncertainty. Table 6.6 summarizes the total uncertainty computed for $D, D_{32}, V_{\text {axial }}$, and $V_{r}$. These are the values used for the uncertainty bars for the corresponding Figure 6.3 and Figure 6.4 presented in Section 6.2.1. Typically the smallest total uncertainties occur at the middle of the spray $\left(R_{s}=0 \mathrm{~cm}\right)$ and increase towards the edge of the spray $\left(R_{s}=2.68 \mathrm{~cm}\right)$. This is most prevalent for the $D$ and $D_{32}$ at $R_{s}=2.68 \mathrm{~cm}$ for the 1.38 bar spray. The $x$ and $y$-axis profiles generally have similar uncertainties between them.

Table 6.6: The total uncertainty of the droplet diameter, axial velocity, and radial velocity at $z=3.81 \mathrm{~cm}$ for the $x$ and $y$-traverses (no $\Delta\left(V_{r}\right)_{\text {total }}$ for the $y$-traverse).

\begin{tabular}{|c|c|c|c|c|c|c|c|c|}
\hline $\begin{array}{c}\boldsymbol{P} \\
(\mathbf{b a r})\end{array}$ & $\begin{array}{c}\boldsymbol{R}_{\boldsymbol{s}} \\
(\mathbf{c m})\end{array}$ & $\begin{array}{c}\Delta(\boldsymbol{D})_{\text {total }_{\boldsymbol{x}}} \\
(\mathbf{u m})\end{array}$ & $\begin{array}{c}\Delta\left(\boldsymbol{D}_{\mathbf{3 2}}\right)_{\text {total }_{\boldsymbol{x}}} \\
(\mathbf{u m})\end{array}$ & $\begin{array}{c}\Delta\left(\boldsymbol{V}_{\text {axial }}\right)_{\text {total }} \boldsymbol{x} \\
(\mathbf{m} / \mathbf{s})\end{array}$ & $\begin{array}{c}\Delta\left(\boldsymbol{V}_{\boldsymbol{r}}\right)_{\text {total }_{\boldsymbol{x}}} \\
(\mathbf{m} / \mathbf{s})\end{array}$ & $\begin{array}{c}\Delta(\boldsymbol{D})_{\text {total }_{\boldsymbol{y}}} \\
(\mathbf{u m})\end{array}$ & $\begin{array}{c}\Delta\left(\boldsymbol{D}_{\mathbf{3 2}}\right)_{\text {total }_{\boldsymbol{y}}} \\
(\mathbf{u m})\end{array}$ & $\begin{array}{c}\Delta\left(\boldsymbol{V}_{\text {axial }}\right)_{\text {total }} \\
(\mathbf{m} / \mathbf{s})\end{array}$ \\
\hline \multirow{3}{*}{1.38} & 0 & 3.82 & 10.8 & 0.472 & 0.00942 & 3.67 & 3.698 & 0.472 \\
\cline { 2 - 9 } & 1.34 & 4.08 & 10.8 & 0.480 & 0.0304 & 4.31 & 4.307 & 0.482 \\
\cline { 2 - 9 } & 2.68 & 12.0 & 15.7 & 0.576 & 0.124 & 8.51 & 8.509 & 0.551 \\
\hline \multirow{3}{*}{4.76} & 0 & 1.39 & 9.09 & 0.681 & 0.0146 & 1.34 & 9.080 & 0.681 \\
\cline { 2 - 9 } & 1.34 & 1.47 & 9.09 & 0.682 & 0.0480 & 1.59 & 9.110 & 0.681 \\
\cline { 2 - 9 } & 2.68 & 2.53 & 9.32 & 0.702 & 0.124 & 3.57 & 9.642 & 0.706 \\
\hline \multirow{3}{*}{4.14} & 0 & 1.37 & 7.04 & 0.662 & 0.0169 & 1.33 & 7.004 & 0.657 \\
\cline { 2 - 9 } & 1.34 & 1.29 & 7.02 & 0.661 & 0.0645 & 1.31 & 7.010 & 0.658 \\
\cline { 2 - 9 } & 2.68 & 1.69 & 7.09 & 0.677 & 0.149 & 2.13 & 7.210 & 0.667 \\
\hline
\end{tabular}

The Sauter mean diameter results presented in Figure $6.2 \mathrm{~b}$ ) noticeably varies by radial location resulting in lower quality data compared to the arithmetic mean diameters in Figure 6.2 a). The variation of $D_{32}$ along $R_{s}$ is due to the larger droplets being weighted more heavily. These larger droplets are more inconsistent in their frequency compared to the smaller droplets. In addition the $D_{32}$ can change by as much as 20 to $25 \%$ if a few larger droplets are filtered out by the PDPA software. Table 6.7 lists the spatial standard deviation of $D_{32}$ across $0 \leq R_{s} \leq 2.68 \mathrm{~cm}$ in Figure 6.2 b). This spatial variation decreases with pressure and is marginally more pronounced in the $y$-axis profile. This observed spatial variation in the measured Sauter mean diameters has not been separately included in the overall estimated uncertainties using Equation 6.3. 
Table 6.7: Spatial standard deviation of $D_{32}$ across $0 \leq R_{s} \leq 2.68 \mathrm{~cm}$ for varying flow conditions at $z=3.81 \mathrm{~cm}$.

\begin{tabular}{|c|c|c|}
\hline$P$ (bar) & $\boldsymbol{s t d}\left(D_{\mathbf{3 2}}\right)_{\boldsymbol{x}}$ (um) & $\boldsymbol{s t d}\left(\boldsymbol{D}_{\mathbf{3 2}}\right)_{\boldsymbol{y}}$ (um) \\
\hline 1.38 & 6.37 & 8.56 \\
\hline 2.76 & 5.09 & 6.84 \\
\hline 4.14 & 5.09 & 6.41 \\
\hline
\end{tabular}

\section{Section 6.2.3: Effects of Varying the Standoff Distance}

As initially reported by Hillen et al. (2013) it was found that variation in the data between $z=$ $3.18,3.81$, and $4.45 \mathrm{~cm}$ was minor. This conclusion is supported by Figure 6.6 which consists of the measured spray droplet arithmetic mean diameters, Sauter mean diameters, and the velocity components for a constant spray condition of 4.14 bar and $54.5 \mathrm{~L} / \mathrm{hr}$. The effects varying $z$ for the given range on the diameter size is shown to be negligible in Figures 6.6 a) and b). Very small deceases in the average axial and radial velocity profiles can be observed in Figures $6.6 \mathrm{c}$ ) and d) as $z$ is increased. The differences are due to the shorter standoff distances being closer to the nozzle exit where the drops have been exposed to less drag due to air resistance, but, again, the variation is minor. The trends shown in Figure 6.6 were similar for the variation of the standoff distances for the lower spray conditions of $1.38 \mathrm{bar}, 29.5 \mathrm{~L} / \mathrm{hr}$ and $2.76 \mathrm{bar}, 43.5 \mathrm{~L} / \mathrm{hr}$ with the corresponding plots presented in Appendix B including the respective average velocity magnitudes. 


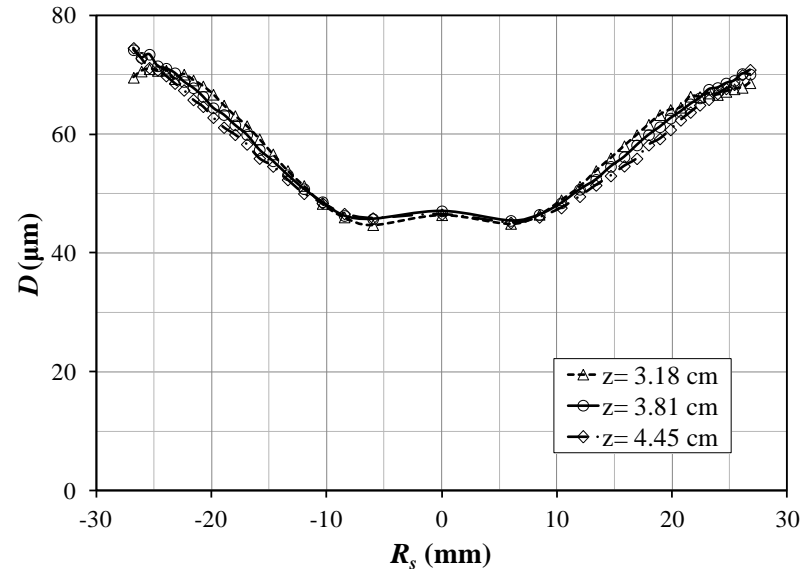

a)

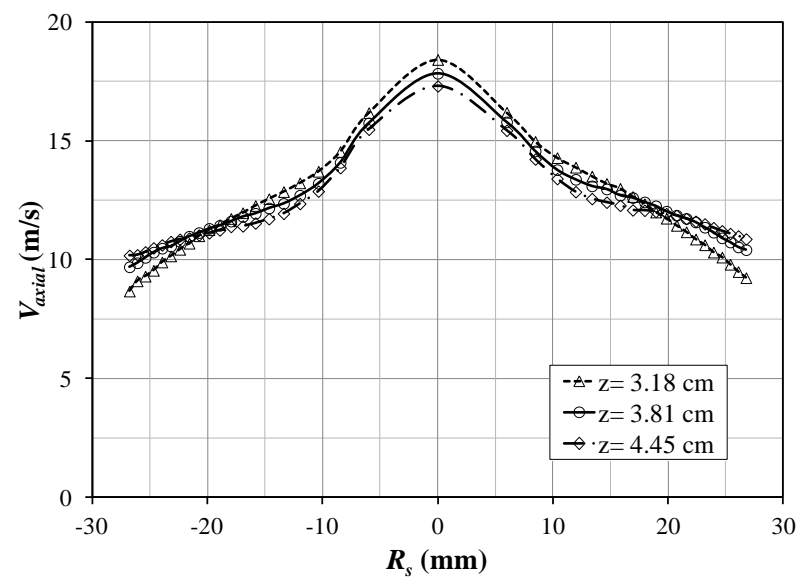

c)

Figure 6.6: Variation of the standoff distance of the measuring profile to the nozzle for a spray flow condition of 4.14 bar and $54.5 \mathrm{~L} / \mathrm{hr}$. a) Arithmetic mean diameters. b) Sauter mean diameters. c) Average axial velocities. d) Average radial velocities.

\section{Section 6.2.4: Spray Nozzle Droplet Size and Velocity Characterization}

Spray droplet and velocity profiles measured using PDPA have been utilized to compute global integrated average values of the dimensionless Weber, Reynolds, and Froude numbers to characterize the sprays at each nozzle pressure. Droplet and velocity data listed in Table 6.8 have been computed via a global average across the span of the spray encompassing every performed spray scenario (such as the example given in Figures 6.2 and 6.3) without the impact surface installed. The global integrated average of the $x$ and $y$-axis mean arithmetic mean diameter, Sauter mean diameter, and axial velocity profiles have been used to compute the values listed. The global 
averages of the spray profiles are consistent with the trends discussed in the previous Sections 6.2.1 and 6.2.2. Note that because the radial traverse locations were selected such that each data point corresponded to an equal (annular) area, this global averaging corresponds exactly to integrating in cylindrical coordinates and dividing by the circular area.

Table 6.8: Global integrated average of spray droplet characteristics across $-2.54 \leq \boldsymbol{R}_{s} \leq \mathbf{2 . 5 4}$ cm.

\begin{tabular}{|c|c|c|c|c|c|c|}
\hline $\boldsymbol{P}(\mathbf{b a r})$ & $\boldsymbol{z}(\mathbf{c m})$ & $\boldsymbol{D}(\boldsymbol{\mu m})$ & $\boldsymbol{D}_{\mathbf{3 2}}(\boldsymbol{\mu m})$ & $\boldsymbol{V}_{\text {axial }}(\mathbf{m} / \mathbf{s})$ & $\boldsymbol{V}_{\boldsymbol{r}}(\mathbf{m} / \mathbf{s})$ & $\boldsymbol{V}(\mathbf{m} / \mathbf{s})$ \\
\hline \multirow{3}{*}{1.38} & 3.18 & 90.8 & 167.7 & 7.36 & 3.25 & 8.18 \\
\cline { 2 - 7 } & 3.81 & 93.9 & 171.6 & 7.55 & 2.78 & 8.13 \\
\cline { 2 - 7 } & 4.45 & 95.1 & 175.1 & 7.51 & 2.34 & 7.93 \\
\hline \multirow{3}{*}{2.76} & 3.18 & 70.5 & 133.9 & 10.4 & 4.41 & 11.5 \\
\cline { 2 - 7 } & 3.81 & 71.0 & 135.7 & 10.5 & 3.68 & 11.3 \\
\cline { 2 - 7 } & 4.45 & 70.6 & 135.7 & 10.2 & 3.04 & 10.8 \\
\hline \multirow{3}{*}{4.14} & 3.18 & 59.3 & 116.2 & 12.6 & 5.18 & 13.8 \\
\cline { 2 - 7 } & 3.81 & 58.7 & 115.2 & 12.6 & 4.28 & 13.4 \\
\cline { 2 - 7 } & 4.45 & 57.5 & 114.3 & 12.4 & 3.53 & 13.0 \\
\hline
\end{tabular}

The dimensionless Weber, Reynolds, and Froude numbers characterizing the spray presented in Table 6.9 have been computed with the global arithmetic and Sauter mean diameters listed in Table 6.8. The arithmetic average diameters gave more weight to the smaller droplets that form the majority of the spray population while the Sauter mean diameter weighted more heavily the larger droplets. The global averages of the mean axial velocity profiles were used in computing the dimensionless numbers in Table 6.9 since the majority of the correlations developed for single drops experiments in the literature including those listed in Table 2.2 are based on the axial velocity. The Weber numbers computed based on $D$ are less than or very near to (at the lower spray flow conditions) the typical Weber number criterion found in Table 2.2 for the onset of splashing while the Weber number values computed based on $D_{32}$ are all above this threshold. 
Table 6.9: Characterization of the spray in terms of the average $W e, R e$, and $F r$ for each spray condition. Dimensionless numbers were computed using the axial velocity component.

\begin{tabular}{|c|c|c|c|c|c|c|c|}
\cline { 2 - 8 } \multicolumn{2}{c|}{} & \multicolumn{3}{c|}{ Calculated with $\boldsymbol{D}$} & \multicolumn{3}{c|}{ Calculated with $\boldsymbol{D}_{\mathbf{3 2}}$} \\
\hline $\boldsymbol{P}$ (bar) & $\boldsymbol{z}(\mathbf{c m})$ & $\boldsymbol{W e}$ & $\boldsymbol{R e}$ & $\boldsymbol{F r}$ & We & $\boldsymbol{R e}$ & $\boldsymbol{F r}$ \\
\hline \multirow{3}{*}{1.38} & 3.18 & 68 & 666 & 60,800 & 125 & 1,230 & 33,000 \\
\cline { 2 - 9 } & 3.81 & 73 & 706 & 61,800 & 134 & 1,290 & 33,800 \\
\cline { 2 - 9 } & 4.45 & 73 & 711 & 60,500 & 135 & 1,310 & 32,800 \\
\hline \multirow{3}{*}{2.76} & 3.18 & 105 & 733 & 158,000 & 200 & 1,390 & 83,000 \\
\cline { 2 - 8 } & 3.81 & 107 & 743 & 158,000 & 205 & 1,420 & 82,900 \\
\cline { 2 - 8 } & 4.45 & 101 & 720 & 151,000 & 195 & 1,380 & 78,600 \\
\hline \multirow{3}{*}{4.14} & 3.18 & 128 & 742 & 272,000 & 252 & 1,460 & 139,000 \\
\cline { 2 - 8 } & 3.81 & 127 & 735 & 275,000 & 250 & 1,440 & 140,000 \\
\cline { 2 - 8 } & 4.45 & 120 & 707 & 271,000 & 239 & 1,410 & 136,000 \\
\hline
\end{tabular}

In addition to the dimensionless numbers based on the global diameter and velocity averages, values have also been computed for the 5\% population of diameters consisting of the largest and the $5 \%$ population consisting of the smallest droplets for each flow condition at the spray center $\left(R_{c}=0\right.$ $\mathrm{mm})$ and edge $\left(R_{c}=2.47 \mathrm{~cm}\right)$. Table 6.10 contains the droplet arithmetic mean and Sauter mean diameters along with the mean velocity components computed from the 5\% of the population consisting of the smallest droplets. These droplets are very small and relatively slow compared to the global averages in Table 6.8.

Table 6.10: Mean drop characteristics calculated from the 5\% population consisting of the smallest droplets at $z=3.81 \mathrm{~cm}$.

\begin{tabular}{|c|c|c|c|c|c|c|}
\hline $\boldsymbol{P}(\mathbf{b a r})$ & $\boldsymbol{R}_{\boldsymbol{S}}(\mathbf{c m})$ & $\boldsymbol{D}(\boldsymbol{\mu m})$ & $\boldsymbol{D}_{\mathbf{3 2}}(\boldsymbol{\mu m})$ & $\boldsymbol{V}_{\text {axial }}(\mathbf{m} / \mathbf{s})$ & $\boldsymbol{V}_{\boldsymbol{r}}(\mathbf{m} / \mathbf{s})$ & $\boldsymbol{V}(\mathbf{m} / \mathbf{s})$ \\
\hline \multirow{2}{*}{1.38} & 0 & 8.68 & 10.0 & 8.68 & 0.0396 & 8.73 \\
\cline { 2 - 7 } & 2.47 & 15.3 & 20.6 & 5.48 & 3.35 & 6.46 \\
\hline \multirow{2}{*}{2.76} & 0 & 8.67 & 9.64 & 12.7 & 0.0877 & 12.8 \\
\cline { 2 - 7 } & 2.47 & 15.9 & 21.5 & 8.36 & 4.78 & 9.71 \\
\hline \multirow{2}{*}{4.14} & 0 & 7.79 & 8.61 & 15.9 & 0.117 & 15.9 \\
\cline { 2 - 7 } & 2.47 & 14.8 & 19.2 & 11.0 & 6.24 & 12.7 \\
\hline
\end{tabular}

The average droplet arithmetic and Sauter mean diameter in Table 6.10 were used to compute the dimensionless numbers found in Table 6.11 for the $5 \%$ population consisting of the smallest droplets. These smallest droplets were generally within the drop rebound/deposition criteria defined 
in Table 2.2 and their mass only contributed up to $0.03 \%$ of the total spray mass. Thus these droplets were of little interest and were not incorporated into the single drop sub-cavity film thickness matrix to be presented in Section 6.5.

Table 6.11: Dimensionless numbers calculated from the 5\% population consisting of the smallest droplets at a constant $z=3.81 \mathrm{~cm}$.

\begin{tabular}{|c|c|c|c|c|c|c|c|}
\cline { 3 - 8 } $\boldsymbol{P}$ (bar) & \multicolumn{3}{c|}{ Calculated with $\boldsymbol{D}$} & \multicolumn{3}{c|}{ Calculated with $\boldsymbol{D}_{\mathbf{3 2}}$} \\
\hline \multirow{2}{*}{1.38} & 0 & 8.96 & 75.0 & 885,000 & 10.3 & 86.6 & 767,000 \\
\cline { 2 - 8 } & 2.47 & 6.29 & 83.5 & 200,000 & 8.45 & 112 & 149,000 \\
\hline \multirow{2}{*}{2.76} & 0 & 19.2 & 110 & $1,910,000$ & 21.4 & 122 & $1,710,000$ \\
\cline { 2 - 8 } & 2.47 & 15.2 & 132 & 448,000 & 20.7 & 179 & 331,000 \\
\hline \multirow{2}{*}{4.14} & 0 & 26.8 & 123 & $3,290,000$ & 29.6 & 136 & $2,980,000$ \\
\cline { 2 - 8 } & 2.47 & 24.5 & 162 & 828,000 & 31.7 & 210 & 640,000 \\
\hline
\end{tabular}

Droplet arithmetic and Sauter mean diameters along with the mean velocity components averaged over the $5 \%$ of the population consisting of the largest droplets are presented in Table 6.12. These droplets are significantly larger and faster than the global Sauter mean droplet diameters listed in Table 6.8, and thus are expected to be significant contributors to cavity formation in the residual liquid film.

Table 6.12: Mean drop characteristics calculated from the 5\% population consisting of the largest droplets at a constant $z=3.81 \mathrm{~cm}$.

\begin{tabular}{|c|c|c|c|c|c|c|}
\hline $\boldsymbol{P}(\mathbf{b a r})$ & $\boldsymbol{R}_{\boldsymbol{s}}(\mathbf{c m})$ & $\boldsymbol{D}(\boldsymbol{\mu m})$ & $\boldsymbol{D}_{\mathbf{3 2}}(\boldsymbol{\mu m})$ & $\boldsymbol{V}_{\text {axial }}(\mathbf{m} / \mathbf{s})$ & $\boldsymbol{V}_{\boldsymbol{r}}(\mathbf{m} / \mathbf{s})$ & $\boldsymbol{V}(\mathbf{m} / \mathbf{s})$ \\
\hline \multirow{2}{*}{1.38} & 0 & 204 & 214 & 10.4 & 0.0799 & 10.4 \\
\cline { 2 - 7 } & 24.7 & 223 & 234 & 8.80 & 5.43 & 10.4 \\
\hline \multirow{2}{*}{2.76} & 0 & 154 & 165 & 15.6 & 0.0910 & 15.6 \\
\cline { 2 - 7 } & 24.7 & 184 & 190 & 12.3 & 7.34 & 14.3 \\
\hline \multirow{2}{*}{4.14} & 0 & 141 & 154 & 19.5 & 0.0934 & 19.5 \\
\cline { 2 - 7 } & 24.7 & 157 & 168 & 14.8 & 8.93 & 17.3 \\
\hline
\end{tabular}

Table 6.13 contains the dimensionless averaged $W e, R e$, and $F r$ numbers computed from the corresponding average data for the 5\% population of the largest spray droplets listed in Table 6.12. This range is also considered to be relevant because these droplets make upwards of $39.3 \%$ of the 
total spray mass. In addition all of these averaged Weber numbers are in the range where cavity formation is expected (see Table 2.2). The highest Weber number computed $(W e=802$ ) has been used as a guide to set the upper limit of the Weber number for the single droplet test matrix to be discussed in Section 6.5.

Table 6.13: Average dimensionless numbers calculated from the $5 \%$ population consisting of the largest droplets at a constant $z=3.81 \mathrm{~cm}$.

\begin{tabular}{|c|c|c|c|c|c|c|c|}
\cline { 2 - 8 } \multicolumn{2}{c|}{} & \multicolumn{3}{c|}{ Calculated with $\boldsymbol{D}$} & \multicolumn{3}{c|}{ Calculated with $\boldsymbol{D}_{\mathbf{3 2}}$} \\
\hline \multirow{2}{*}{1.38} & 0 & 302 & 2,110 & 54,200 & 318 & 2,220 & 51,500 \\
\cline { 2 - 8 } & 24.7 & 244 & 2,020 & 34,400 & 249 & 2,050 & 33,800 \\
\hline \multirow{2}{*}{2.76} & 0 & 512 & 2,390 & 160,000 & 549 & 2,560 & 149,000 \\
\cline { 2 - 8 } & 24.7 & 381 & 2,250 & 83,600 & 393 & 2,320 & 81,000 \\
\hline \multirow{2}{*}{4.14} & 0 & 734 & 2,740 & 275,000 & 802 & 2,990 & 252,000 \\
\cline { 2 - 8 } & 24.7 & 471 & 2,310 & 143,000 & 503 & 2,470 & 133,000 \\
\hline
\end{tabular}

\section{Section 6.3: Splash Results and Discussion: Spray Impact Surface Installed}

This section focuses only on the splashed droplets generated by the spray impinging onto the installed impact surface. These results are expected to have no influence on the single drop test matrix presented in Section 6.5, but are documented for use in future work.

\section{Section 6.3.1: Comparison of Spray with and without the Impact Surface Installed}

A comparison was made in Figure 6.7 between the spray profiles measured at $z=3.81 \mathrm{~cm}$ presented in Section 6.2 without the impact surface installed and the spray droplet profiles that were extracted from the PDPA data measured at $z=3.65 \mathrm{~cm}$ with the impact surface installed. Good agreement for a flow condition of $1.38 \mathrm{bar}, 29.5 \mathrm{~L} / \mathrm{hr}$ can be observed in Figures $6.7 \mathrm{a}$ ) and b). At the two higher flow conditions of $2.76 \mathrm{bar}, 43.5 \mathrm{~L} / \mathrm{hr}$ and $4.14 \mathrm{bar}, 54.5 \mathrm{~L} / \mathrm{hr}$ the arithmetic mean diameter with the installed impact surface can vary as much as 15 and $21 \mu \mathrm{m}$, respectively, compared to the basic spray data. Also, in Figure 6.7 a) the diameter data for installed target spray is nearly constant for the two highest spray flow conditions and nearly identical. The velocity 
components of the impact surface spray data in Figures $6.7 \mathrm{c}$ ) and d) are in better agreement with the basic spray and follow similar trends, though there is still noticeable variation.
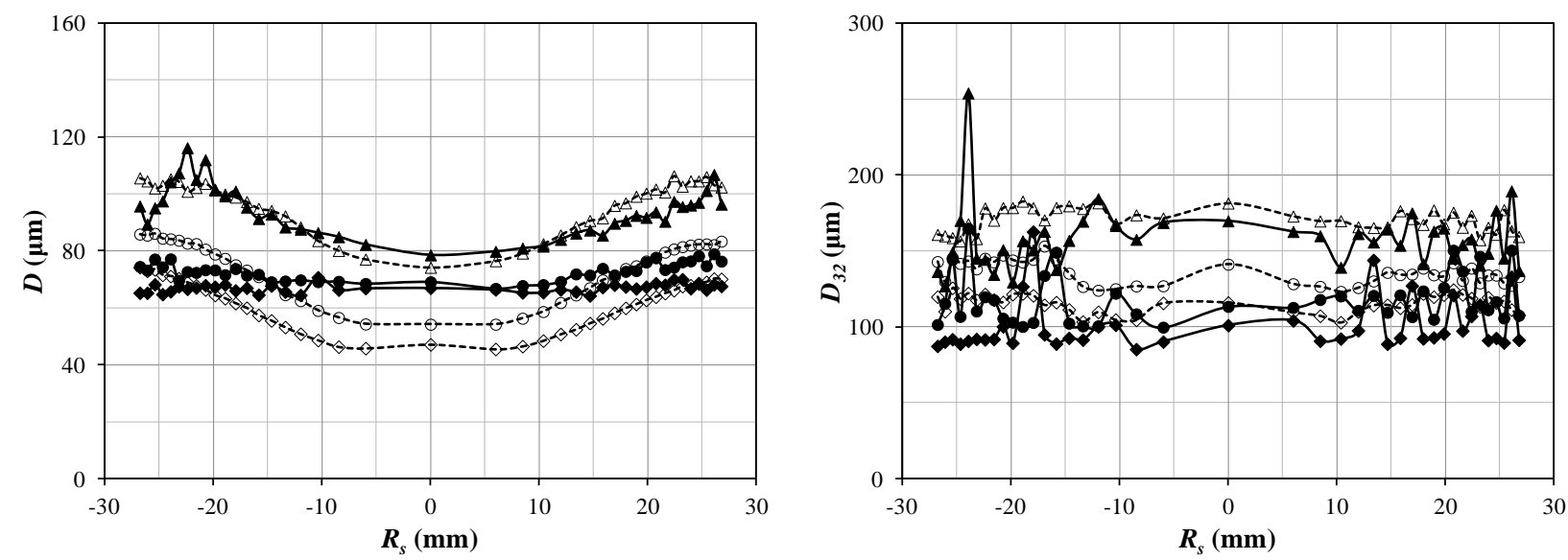

a)

b)
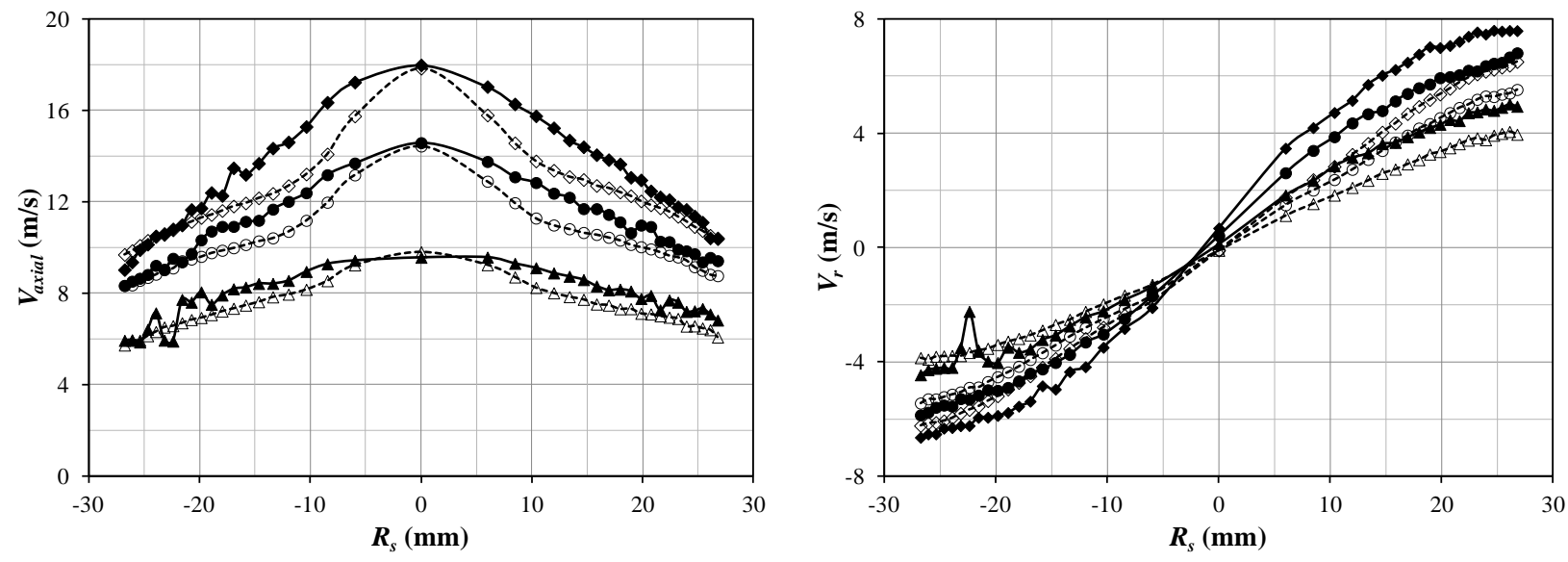

d)

Figure 6.7: Comparison of the spray droplet characteristics with $(z=3.60)$ and without $(z=$ 3.81) the impact surface installed for 1.38 bar, 2.76 bar, 4.14 bar. a) Arithmetic mean diameter. b) Sauter mean diameter. c) Mean axial velocity. d) Mean radial velocity. $\leftarrow-1.38$ bar, 29.5 L/hr, with impact surface; $-2.76 \mathrm{bar}, 43.5 \mathrm{~L} / \mathrm{hr}$, with impact surface; $-4.14 \mathrm{bar}, 54.5 \mathrm{~L} / \mathrm{hr}$, with impact surface; $-\backsim-1.38$ bar, $29.5 \mathrm{~L} / \mathrm{hr}$, without impact surface; - - - $2.76 \mathrm{bar}, 43.5 \mathrm{~L} / \mathrm{hr}$, without impact surface; $\diamond-4.14 \mathrm{bar}, 54.5 \mathrm{~L} / \mathrm{hr}$ without impact surface.

It is believed that the variations and disagreements found in Figure 6.7 are due to, at least in part, the denser spray region caused by the splashed droplets when the spray is impinging onto the impact surface. As previously discussed in Section 3.5.1, the quality of PDPA data can be affected by large droplet fluxes, which is why the best agreement is in the sparser spray at the lowest flow settings. An additional contributing factor, also discussed in Section 3.5.1, is the smaller population 
on which to base the statistics for the impact surface spray since they are extracted from the overall PDPA data consisting of both the incoming spray droplets and the splashed droplets. Another plausible contributing factor could be the increased droplet-to-droplet interactions between the impinging spray droplets and the ejected splashed droplets. The abnormal spikes in data located between $-25<R_{s}<-20 \mathrm{~mm}$ for every plot presented in Figure 6.7 for the $1.38 \mathrm{bar}, 29.5 \mathrm{~L} / \mathrm{hr}$ impact surface spray are believed to be a combination of the aforementioned lower statistics and a not fully

formed nozzle spray cone at the lowest pressure (see Section 6.2.1). Appendix C contains the corresponding comparison of the velocity magnitude between the basic spray and impact surface spray.

\section{Section 6.3.2: Separated Splashed Droplet Components: Impact surface Installed}

The PDPA data collected for a spray impinging onto the impact surface has been separated into their respective incoming spray and splashed droplet components as described in Section 3.5. Figure 6.8 depicts the arithmetic and Sauter mean diameters of the separated data for a nozzle flow condition of 4.14 bar, $54.5 \mathrm{~L} / \mathrm{hr}$ in terms of the original spray, ejected splash, returning splash, and absolute splash droplets at $z=3.65 \mathrm{~cm}$. Consistent with Kalantari and Tropea (2006) it was found that the splashed droplet sizes were the same order of magnitude as the spray droplets at all radial locations as shown in the respective arithmetic and Sauter mean diameters in Figures 6.8 a) and b). As discussed previously in Section 2.3.3 and observed experimentally, single drop impacts generally produce multiple splash droplets that are considerably smaller than the initial drop. Plausible contributors to the larger splash drop sizes include rebounding drops on the spray film liquid, and Worthington jet droplets, which are on the same size scale as the impinging droplet (see Section 2.3.3). Additional possible contributors include thicker crowns due to neighboring droplet impingement interactions, ejected film ligaments, and the droplet impingement angle (Kalantari and 
Tropea, 2006). Another possible explanation includes drop-to-drop interactions where the splash droplets could merge forming larger droplets.

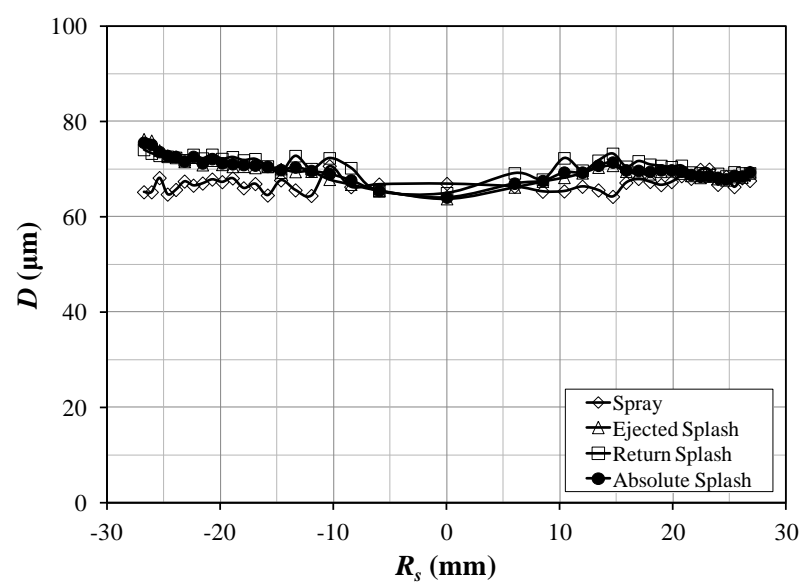

a) surface installed for a flow condition of $4.14 \mathrm{bar}, 54.5 \mathrm{~L} / \mathrm{hr}$ at a constant $z=3.65 \mathrm{~cm}$. a) Arithmetic mean diameter. b) Sauter mean diameter.

Axial and radial mean velocities for the separated spray and splashed droplet components are shown in Figures 6.9 a) and b) respectively. As seen in Figure 6.9 a) the ejected splashed droplet mean axial velocities never exceed $2.5 \mathrm{~m} / \mathrm{s}$ consistent with Kalantari and Tropea (2006) in which axial velocities were not observed exceeding $3 \mathrm{~m} / \mathrm{s}$. The returning splashed droplet mean axial velocity is comparable in magnitude to the ejected, yet it is noticeably slower with a maximum nominal mean axial velocity magnitude of $1.5 \mathrm{~m} / \mathrm{s}$. There was not a distinguishing difference in the radial velocity component observed between the different splashing types with measured mean radial velocities increasing nominally from 1.8 to $5 \mathrm{~m} / \mathrm{s}$, as shown in Figure 6.9 b). However, because radial splashed droplet velocities are similar in size to axial components, this signifies that the splashed droplets generally have a low trajectory angle with respect to the impact surface. Examining Figures 6.9 a) and b), increases in the ejected droplet axial velocity component correspond to a decreasing radial velocity component towards the spray centerline. This indicates that the splash droplets in this region are ejecting at greater angles from the surface. These results are 
also consistent with those found in Kalantari and Tropea (2006). The spray, ejected splash, return splash, and absolute splash droplets for the flow conditions of $1.38 \mathrm{bar}, 29.5 \mathrm{~L} / \mathrm{hr}$ and $2.76 \mathrm{bar}, 43.5$ $\mathrm{L} / \mathrm{hr}$ followed identical trends and behaviors to those described for Figures 6.8 and 6.9. The corresponding plots comparing the spray droplets and the various splash droplet categories for these two lower nozzle flow conditions are presented in Appendix C along with the corresponding velocity magnitude plots.

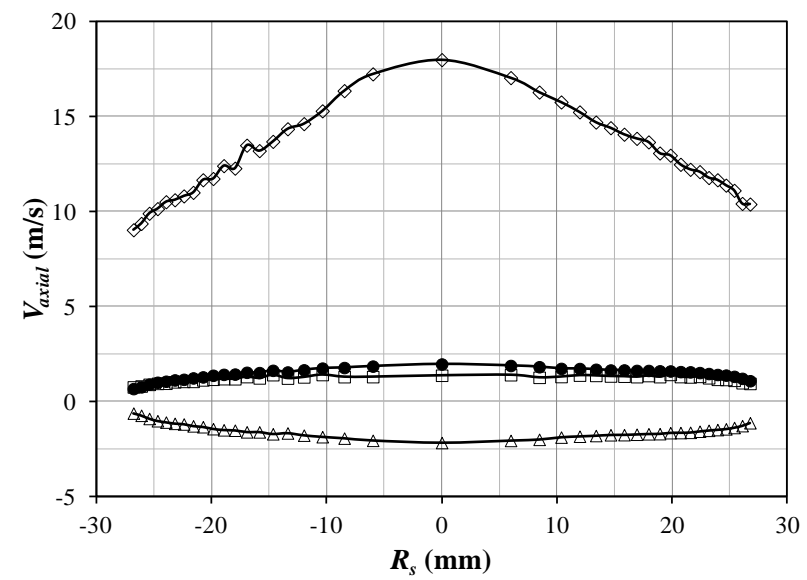

a)

Figure 6.9: Droplet velocities of the spray, ejected, return, and absolute splash data with the impact surface installed for $4.14 \mathrm{bar}, 54.5 \mathrm{~L} / \mathrm{hr}$ at a constant $z=3.65 \mathrm{~cm}$. a) Mean axial velocity, b) Mean radial velocity. $\checkmark$ Incoming spray droplets, $\checkmark$ ejected splashed droplets, $\neg$ - returning splashed droplets, $\rightarrow$ - absolute splashed droplets.

Table 6.14 presents the global integrated average of the standard deviation for each measuring location of the splashed droplet characteristics. There is a large variance in the droplet diameter data suggesting that the population does contain the expected smaller splash droplets. 
Table 6.14: The averaged standard deviation of the splash droplet characteristics averaged across $-2.54<R_{s}<2.54 \mathrm{~cm}$ at $z=3.65 \mathrm{~cm}$.

\begin{tabular}{|c|c|c|c|c|c|}
\hline Splash Type & $\boldsymbol{P}(\mathbf{b a r})$ & $\boldsymbol{s t d}(\boldsymbol{d})(\boldsymbol{\mu m})$ & $\boldsymbol{s t d}\left(\boldsymbol{v}_{\text {axial }}\right)(\mathbf{m} / \mathbf{s})$ & $\boldsymbol{s t d}\left(\boldsymbol{v}_{\boldsymbol{r}}\right)(\mathbf{m} / \mathbf{s})$ & $\boldsymbol{s t d}(\boldsymbol{v})(\mathbf{m} / \mathbf{s})$ \\
\hline \multirow{3}{*}{ Ejected } & 1.38 & 58.1 & 1.04 & 1.37 & 1.42 \\
\cline { 2 - 6 } & 2.76 & 42.1 & 1.43 & 1.60 & 1.76 \\
\cline { 2 - 6 } & 4.14 & 38.8 & 1.39 & 1.74 & 1.85 \\
\hline \multirow{3}{*}{ Return } & 1.38 & 58.5 & 1.00 & 1.32 & 1.37 \\
\cline { 2 - 6 } & 2.76 & 42.9 & 1.38 & 1.57 & 1.73 \\
\cline { 2 - 6 } & 4.14 & 38.7 & 1.34 & 1.72 & 1.82 \\
\hline \multirow{3}{*}{ Absolute } & 1.38 & 54.4 & 1.28 & 1.88 & 2.02 \\
\cline { 2 - 6 } & 2.76 & 40.9 & 1.46 & 1.95 & 2.11 \\
\cline { 2 - 6 } & 4.14 & 37.1 & 1.50 & 1.95 & 2.09 \\
\hline
\end{tabular}

\section{Section 6.3.3: Effects of Varying the Nozzle Flow Conditions on the Splashed Droplet Components}

Since all three splashed droplet types follow similar trends, the absolute splash droplets are utilized in Figure 6.10 to demonstrate the influence of spray flow conditions on the splashed droplet behavior. Figures 6.1 a) and b) show that the spray flow conditions have a negligible effect on the arithmetic and Sauter mean droplet diameters except for the $1.38 \mathrm{bar}, 29.5 \mathrm{~L} / \mathrm{hr}$ case where the Sauter mean droplet diameter is nominally $63 \mu \mathrm{m}$ larger. The lack of variation in the droplet mean size could be attributed to the lack of adequate quality in the statistical data available for the PDPA spray impact surface data. The flow conditions also have a nearly negligible effect on the mean axial velocity of the splashed droplets with a maximum difference of $0.13 \mathrm{~m} / \mathrm{s}$ for the example shown in Figure $6.10 \mathrm{c}$ ). Of the available data the only definitive splashed droplet trend observed with increasing the spray pressure and flow rate was for the mean radial velocity shown in Figure $6.10 \mathrm{~d}$ ). Towards the spray edge the mean radial velocity of the splash droplets increases with the nozzle pressure and flow rate. This is reasonable since the spray cone angle increases from 1.38 bar to 4.14 bar resulting in the impinging droplets to have a smaller impact angle, resulting in a lower trajectory for the generated splash droplets. Plots similar to those presented in Figure 6.10 are contained in 
Appendix $\mathrm{C}$ for the ejected and returning spray splash types in addition to the variation of the flow conditions for velocity magnitude for all three spray splash types.

In all of these plots there was a dramatic fluctuation in the data for the $1.38 \mathrm{bar}, 29.5 \mathrm{~L} / \mathrm{hr}$ spray conditions at radial measurements of $R_{s}<-20 \mathrm{~mm}$ as shown in Figure 6.10. It is believed that a combination of the spray cone not being fully developed in that region and a reduced quality in the statistical data was the source.
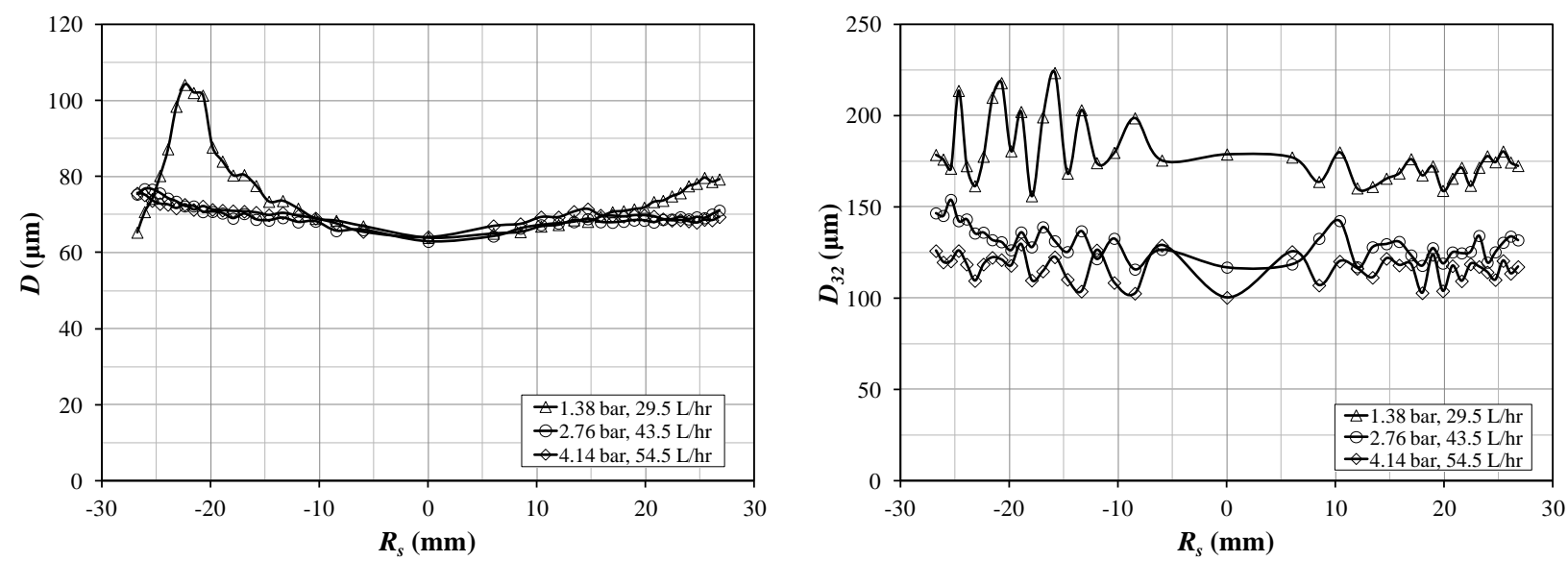

a)

b)
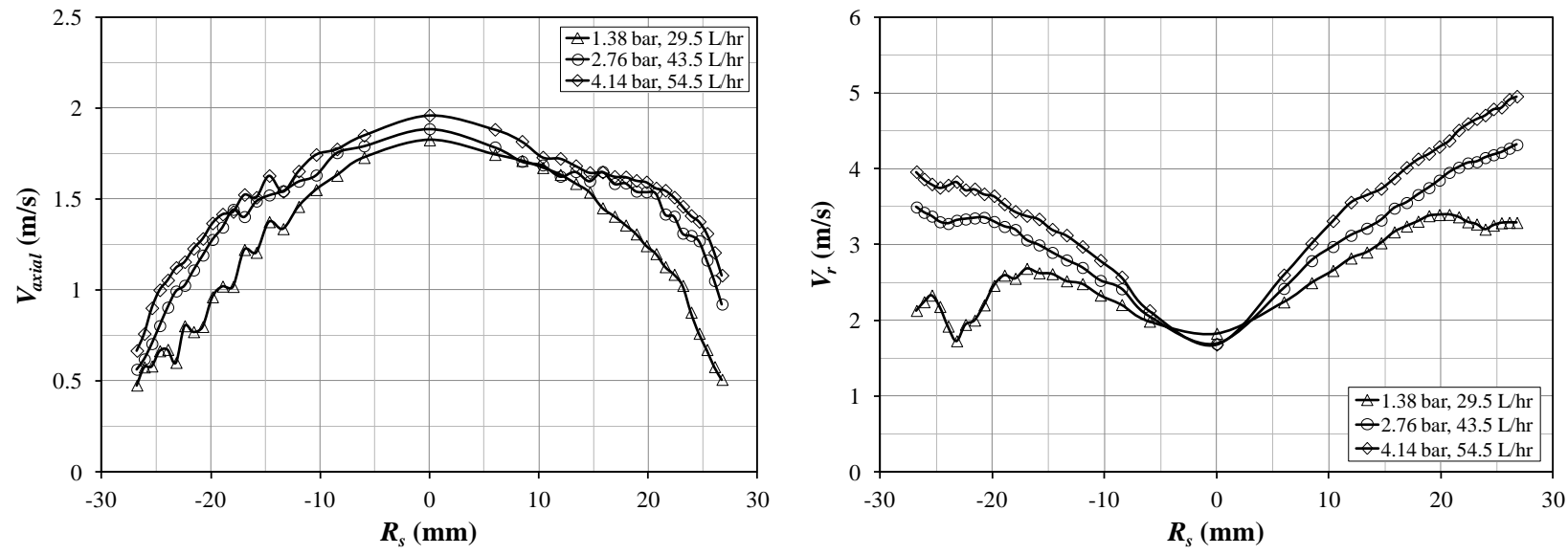

d)

c)

Figure 6.10: Absolute splash droplet characteristics at varying pressures at a constant $z=36.5$ mm. a) Arithmetic mean droplet diameter. b) Sauter mean droplet diameter. c) Mean axial velocity. d) Mean radial velocity. 


\section{Section 6.3.4: Comparison of $x$-axis Splash Results with Vertical Edge Splash Results}

To check the validity of the splashed droplet diameter and velocity profiles measured radially above the impact surface at $z=3.65 \mathrm{~cm}$ additional measurements were performed for traverses along the $z$ direction on the $x z$-plane at a radius just beyond the spray edge as described in Section 3.5.1. Because two symmetrical traverses were performed at a constant $R_{s}= \pm 2.68 \mathrm{~cm}$, the values shown in Figure 6.11 were computed according to $\Psi\left(z_{i}\right)=1 / 2\left[\Psi_{x=R_{s}}\left(z_{i}\right)+\Psi_{x=R_{s}}\left(z_{i}\right)\right]$, with appositive radial velocity defined as away from the spray centerline. Figures 6.1 a) and b) show splash droplet sizes of nominally $55<D<76 \mu \mathrm{m}$, and $90<D_{32}<170 \mu \mathrm{m}$. All the vertical profiles collected at $R_{s}=2.68$ $\mathrm{cm}$ and $R_{s}=-2.68 \mathrm{~cm}$ showed excellent symmetry. These data are in good agreement with those presented in Figure 6.8 and Figure 6.10 of nominally $60<D<80 \mu \mathrm{m}$, and $100<D_{32}<180 \mu \mathrm{m}$, respectively. Data collected above the impact surface level show that the mean diameters decrease with increasing spray pressure and flow rate by as much as $16 \mu \mathrm{m}$ as shown in Figure 6.11. Additional observations of Figure 6.11 a) show that while approaching the impact surface plane, the arithmetic mean splashed diameters converge to nominally $61 \mu \mathrm{m}$ at about $z=3.25 \mathrm{~cm}$ before increasing to nominally $74 \mu \mathrm{m}$ near the surface. Sauter mean diameters shown in Figure $6.11 \mathrm{~b}$ ) demonstrate a similar trend as they also nearly converge at $z=3.65 \mathrm{~cm}$.

Figure $6.11 \mathrm{c}$ ) shows that the splashed droplet axial velocities remain within the range of -1.5 $\mathrm{m} / \mathrm{s}$ to $-1.25 \mathrm{~m} / \mathrm{s}$ between $1.75<z<3.25 \mathrm{~cm}$ for the two extremes of spray pressure and flow rate before rapidly increasing to nominally $3 \mathrm{~m} / \mathrm{s}$ due to the nozzle overspray of the impact surface. Ligaments from the ejecting residual spray film flow were also suspected to contribute to the spike in data near the impact surface plane. The low splashed droplet trajectory is evident in the mean droplet radial velocities shown in Figure 6.11 d). There is a rapid rise in the mean radial velocities, where the spray flow conditions of 1.38 bar, 2.76 bar, and 4.14 bar reach respective peaks of 3.34 , 4.27 , and $5.17 \mathrm{~m} / \mathrm{s}$ at nominally $z=3.50 \mathrm{~cm}$. Mean axial velocities between $1.75<z<3.25 \mathrm{~cm}$ and 
the mean radial velocities near the impact surface plane are consistent with those measured radially $1.6 \mathrm{~mm}$ above the impact surface shown in Figures 6.8 and 6.1. As expected, the splashed droplet velocities depicted in Figure 6.11 rapidly approach zero for the measurements beneath the impact surface plane. The corresponding splashed droplet average velocity magnitude data for the flow conditions in Figure 6.11 is located in Appendix C.
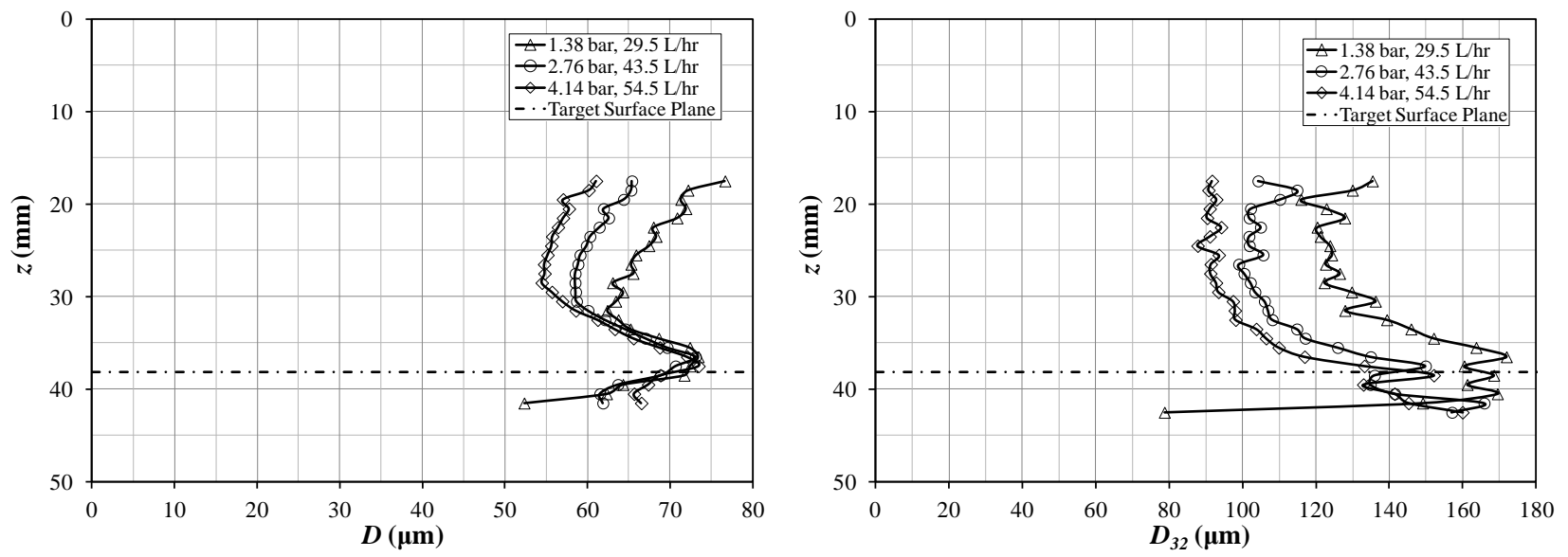

a)

b)
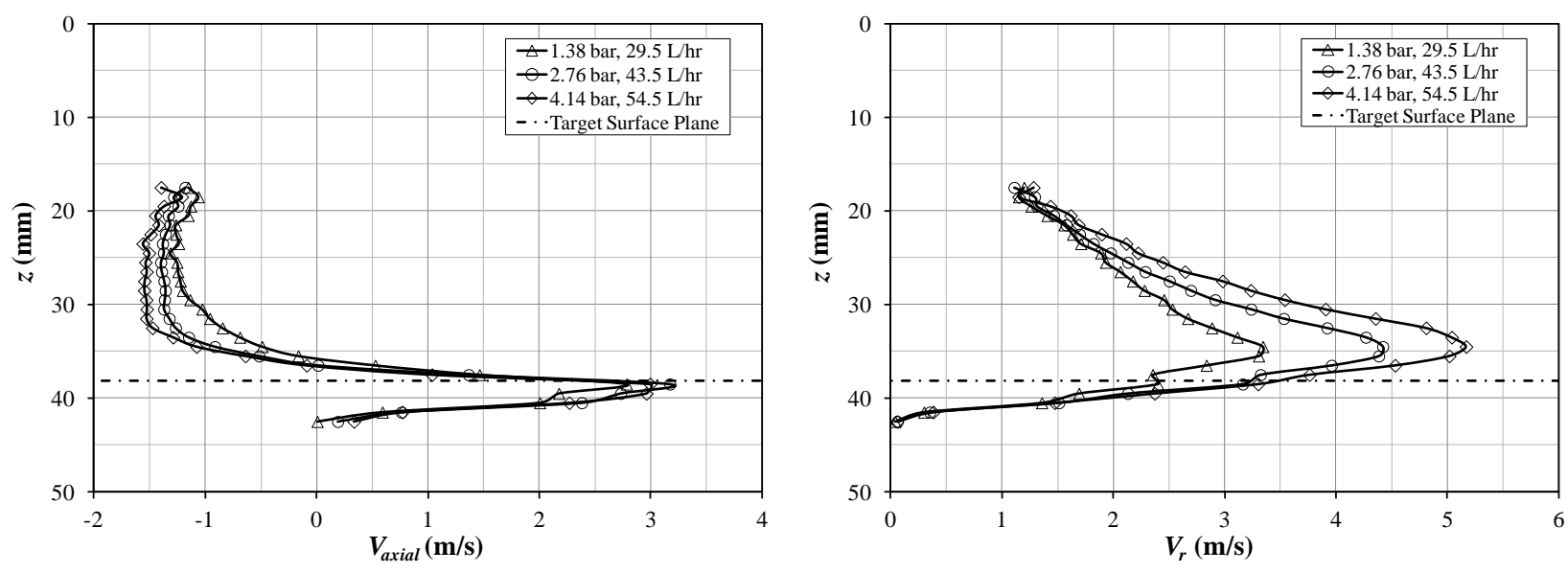

d)

Figure 6.11: Vertical PDPA results of the splashed droplets at $\boldsymbol{R}_{s}= \pm 2.68 \mathrm{~cm}$. a) Arithmetic mean diameter, b) Sauter mean diameter, c) Mean axial velocity, d) Mean radial velocity.

\section{Section 6.3.5: Splashed Droplets Characterized in Dimensionless Numbers}

Global integrated averages of the ejected, return, and absolute spray splashed droplet characteristics measured radially above the impact surface $(z=3.65 \mathrm{~cm})$ are listed in Table 6.15 for 
varying flow conditions. The global average results confirm the lack of significant correlations between spray flow conditions and the splashed droplet average size and axial velocity. The radial velocity and thus the velocity magnitude listed in Table 6.15 do increase with increasing nozzle pressure and flow rate. Sauter mean diameter decreases with increasing pressure.

Table 6.15: Global integrated averages of the splashed droplet characteristics at $z=3.65 \mathbf{c m}$.

\begin{tabular}{|c|c|c|c|c|c|c|}
\hline Splash Type & $\boldsymbol{P}(\mathbf{b a r})$ & $\boldsymbol{D}(\boldsymbol{\mu m})$ & $\boldsymbol{D}_{\mathbf{3 2}}(\boldsymbol{\mu m})$ & $\boldsymbol{V}_{\text {axial }}(\mathbf{m} / \mathbf{s})$ & $\boldsymbol{V}_{\boldsymbol{r}}(\mathbf{m} / \mathbf{s})$ & $\boldsymbol{V}(\mathbf{m} / \mathbf{s})$ \\
\hline \multirow{3}{*}{ Ejected } & 1.38 & 75.9 & 196 & 0.984 & 2.42 & 2.82 \\
\cline { 2 - 7 } & 2.76 & 69.2 & 135 & 1.27 & 3.25 & 3.73 \\
\cline { 2 - 7 } & 4.14 & 69.6 & 123 & 1.25 & 3.80 & 4.21 \\
\hline \multirow{3}{*}{ Return } & 1.38 & 76.8 & 196 & 0.960 & 2.29 & 2.68 \\
\cline { 2 - 7 } & 2.76 & 69.9 & 137 & 1.22 & 3.14 & 3.61 \\
\cline { 2 - 7 } Absolute & 4.14 & 70.7 & 121 & 1.20 & 3.67 & 4.08 \\
\hline & 1.38 & 76.0 & 179 & 1.16 & 2.66 & 3.12 \\
\cline { 2 - 7 } & 2.76 & 69.4 & 130 & 1.38 & 3.29 & 3.82 \\
\cline { 2 - 7 } & 4.14 & 69.9 & 116 & 1.47 & 3.67 & 4.21 \\
\hline
\end{tabular}

The computed average dimensionless Weber, Reynolds, and Froude numbers for the three splash types presented in Table 6.16 were computed for the corresponding arithmetic and Sauter mean diameters listed Table 6.15. The axial velocities from Table 6.15 were utilized to compute the splash dimensionless numbers consistent with the reasoning used for those presented in Table 6.16. The splashed droplets are not expected to make any significant contribution to cavity formation on the residual spray film. This is because the computed Weber numbers are all well below or near the rebound/deposition criteria presented in Table $2.2(W e<3.0, W e=20$, and $W e<30)$. 
Table 6.16: Dimensionless numbers computed from the global average characteristics of the ejected, return, and absolute splashed droplets at $z=3.65 \mathrm{~cm}$.

\begin{tabular}{|c|c|c|c|c|c|c|c|}
\cline { 3 - 8 } \multicolumn{2}{c|}{} & \multicolumn{3}{c|}{ Calculated with $\boldsymbol{c}$} & \multicolumn{3}{c|}{ Calculated with $\boldsymbol{D}_{\mathbf{3 2}}$} \\
\hline Splash Type & $\boldsymbol{P}$ (bar) & $\boldsymbol{W e}$ & $\boldsymbol{R e}$ & $\boldsymbol{F r}$ & $\boldsymbol{W e}$ & $\boldsymbol{R} \boldsymbol{e}$ & $\boldsymbol{F r}$ \\
\hline \multirow{3}{*}{ Ejected } & 1.38 & 1.01 & 74.4 & 1,300 & 2.61 & 193 & 503 \\
\cline { 2 - 9 } & 2.76 & 1.52 & 87.4 & 2,360 & 2.96 & 170 & 1,210 \\
\cline { 2 - 9 } & 4.14 & 1.49 & 86.6 & 2,280 & 2.63 & 153 & 1,290 \\
\hline \multirow{3}{*}{ Return } & 1.38 & 0.971 & 73.5 & 1,220 & 2.48 & 188 & 479 \\
\cline { 2 - 9 } & 2.76 & 1.43 & 85.0 & 1,270 & 2.79 & 166 & 1,110 \\
\cline { 2 - 9 } & 4.14 & 1.40 & 84.5 & 2,080 & 2.40 & 145 & 1,210 \\
\hline \multirow{3}{*}{ Absolute } & 1.38 & 1.39 & 87.5 & 1,800 & 3.28 & 206 & 764 \\
\cline { 2 - 8 } & 2.76 & 1.80 & 95.1 & 2,780 & 3.38 & 178 & 1,490 \\
\cline { 2 - 8 } & 4.14 & 2.06 & 102 & 3,130 & 3.41 & 169 & 1,890 \\
\hline
\end{tabular}

\section{Section 6.4: Volume Flux}

Volume fluxes measured by PDPA were consistently off (low) by nominally a factor of 5 .

This was low relative to what one would compute by dividing the total spray volume flow rate by the spray cross sectional area. PDPA systems are very effective at collecting statistical information on individual particle size and speed; however, achieving mass conservation in the volume flux is difficult. The difficulty is due to the dependency of flux measurements on the droplet size, number density, and velocity which vary from point to point in a spray. Furthermore the number of particles that can be measured in the measuring volume is limited in dense sprays resulting in unreliable droplet number densities (Bade and Schick, 2009, Schwarzkopf et al., 2006, and Widmann et al. 2001).

Improved mass flux measurements require extensive optimization of the PDPA system settings, in which the present author had limited experience and access to the PDPA system. For example, Schwarzkopf et al. (2006) suggested using higher PMT voltages to achieve partial intensity saturation to make the system more sensitive to smaller droplets. Even with the correct settings mass conservation is typically no better than to within 30\% (Schwarzkopf et al., 2006). Another PDPA manufacturer, Artium, has proprietary auto setup software that can more accurately determine the 
volume flux distribution, but the present work did not have access to this capability (Bade, 2009).

The data acquisition software used a Probe Volume Correction (PVC) algorithm to attempt to improve the spray flux measurements. PVC scales the measuring volume to the diameter size to weight the smaller droplets more heavily (Widmann et al. 2001).

\section{Section 6.5: Development of Test Matrix for Single Drop Sub-cavity Experiments}

The single drop sub-cavity test matrix was originally presented in Hillen et al. (2013). Formulation of the detailed test matrix has been based on the dimensionless spray droplet parameters listed in Tables 6.9 and 6.1. The initial target droplet Weber and Reynolds number values selected for study in the single drop experiments to cover the relevant range of spray drop conditions are listed in Table 6.17.

Table 6.17: Single drop experiment dimensionless droplet parameters (Hillen et al., 2013).

\begin{tabular}{|c|c|c|c|}
\hline Case & $\boldsymbol{W e}$ & $\boldsymbol{R e}$ & $\boldsymbol{F r}$ \\
\hline 1 & 140 & 1,210 & 49,800 \\
\hline 2 & 400 & 2,270 & 94,000 \\
\hline 3 & 600 & 2,570 & 193,000 \\
\hline 4 & 800 & 2,800 & 324,000 \\
\hline 5 & 1,000 & 3,340 & 313,000 \\
\hline
\end{tabular}

Initial dimensionless film thicknesses were determined by utilizing the range of values of $h_{0}{ }^{*}$ for the spray experiments listed in Table 2.3. Taken into consideration was the desired 3 to $3.45 \mathrm{~mm}$ range of the single droplet diameter. A minimum attainable initial liquid film thickness of $0.520 \mathrm{~mm}$ eliminated some of the smallest ranges of $h_{0}{ }^{*}$. Since the desired drop size is only slightly less than the maximum CHR measuring range, values of $h_{0}{ }^{*}>1$ were excluded. This was actually not a significant problem since one rapidly approaches a deep pool impact behavior as the value of $h_{0}{ }^{*}$ increases above 1.0. A thin film would not be formed during the impact on a deep pool. These considerations resulted in selection of values of $h_{0}{ }^{*}=1.0,0.5$, and 0.2 for each Case listed in Table 6.17 . 
One limitation of the present work was that matching the single drop Froude numbers with those of the full spray was not possible with the experimental facility at hand. This is because the drops producible for the single drop experiments were on a millimeter scale while the spray droplets were on a scale of 50 to $100 \mu \mathrm{m}$, which referring to Equation 1.3, resulted in unattainably high Froude numbers.

Since the spray droplets are much smaller than those producible by the current experimental apparatus, a water-glycerol solution was used as the working fluid for the single drop experiments in order to match the Reynolds numbers from Table 6.17. A 46.2\% glycerol in water solution by mass of glycerol allowed the Weber and Reynolds numbers of the single droplets to be matched within 5\% of the target values given in Table 6.17. Table 6.18 contains the properties of this glycerol-water solution computed using empirical correlations developed by Cheng (2008) and a detailed collection of databases for glycerol-water solutions (Glycerine Producers' Association, 1963). The correlations in Cheng (2008) produced values consistent with the available databases in Glycerine Producers' Association (1963).

Table 6.18: Computed physical properties of $46.2 \%$ by mass glycerol-water solution for $T=$ $20^{\circ} \mathrm{C}$.

\begin{tabular}{|c|c|c|}
\hline $\boldsymbol{\mu}\left(\mathbf{N} * \mathbf{s} / \mathbf{m}^{2}\right)$ & $\boldsymbol{\rho}\left(\mathbf{k g} / \mathbf{m}^{\mathbf{3}}\right)$ & $\boldsymbol{\sigma}(\mathbf{N} / \mathbf{m})$ \\
\hline 0.00493 & 1120 & 0.0699 \\
\hline
\end{tabular}

Solutions were mixed based on the corresponding volume fraction using a series of graduated cylinders. Volumes were attainable to within a $0.2 \mathrm{~mL}$ accuracy based on a syringe with uncertainty readings of nominally $\pm 0.052 \mathrm{~mL}$. To ensure the droplet and target pool had the same concentration, a single batch of the solution was prepared for both the droplet generator and tank initial liquid thicknesses.

Additional detail is provided in Appendix D for the methodology utilized to determine the mass fraction of glycerol needed to provide the correct fluid properties to match the dimensionless 
numbers in Table 6.17. Appendix D also contains more detail for obtaining the physical properties of the glycerol-water solution in Table 6.18.

Target single droplet diameters of $3 \mathrm{~mm}$ were selected for Cases 1 and 3 in Table 6.17 while a diameter of $3.45 \mathrm{~mm}$ was targeted for Cases 2, 4, and 5. The larger diameter was required for Case 2 to obtain a Reynolds number within $5 \%$ of the target, but it was also used for Cases 4 and 5 to produce the necessary larger dimensionless numbers at a lower drop height than that required for a 3 mm droplet. The lower drop heights for Cases 4 and 5 made the droplet less susceptible to air currents reducing the variation of the droplet impact location by $50 \%$. Additionally, after examining the sub-cavity film thickness results for at $h_{0}{ }^{*}=1.0$ for Case 2 it was determined that Cases 4 and 5 should be performed at an $h_{0}{ }^{*}=0.9$ instead of $h_{0}{ }^{*}=1.0$. Noise was observed for Case 2 that occurred during the initial droplet impingement and retraction phases due to the thickness being beyond the CHR sensor's measuring range. By lowering $h_{0}{ }^{*}$ for Cases 4 and 5, the CHR sensor could measure more data during these time, thus reducing the noise. 


\section{CHAPTER 7: SINGLE DROP RESUltS AND DisCUSSION}

\section{Section 7.1: Introduction}

This chapter focuses on the single drop sub-cavity results for experiments and methodologies described in Chapters 4 and 5. Some of the results contained herein were initially reported by Hillen et al. (2012) and Hillen et al. (2013). Preliminary sub-cavity experimental results from Hillen et al. (2012) are discussed in Section 7.2 for the single drop parameters listed in Table 4.1. This section includes histories of the bottom crown radius, crown height, and centerline film thickness. Also included in this section are sample comparisons between the experimental results and CFD simulations performed by Dinc and Gray in Hillen et al. (2012). The remaining portion of this chapter focuses on the single drop sub-cavity results for the target test matrix discussed in Section 6.5 relevant to the spray of interest.

Section 7.3 documents the actual average single droplet parameters measured experimentally for every target test case listed in Table 6.17 at $h_{0}{ }^{*}=0.2,0.5$, and 1.0. These dimensionless numbers apply to the remaining single drop results in Sections 7.4 - 7.8 and should be used for any future comparisons.

Side-view and bottom-view sub-cavity radius versus time results are presented in Section 7.4. The influence of initial droplet parameters (We and $h_{0}{ }^{*}$ ) on the sub-cavity radius trends are investigated. Also, comparisons between the two methods and the sub-cavity liquid film thickness contour are made.

Flow visualization of the cavity for the different single drop parameters are presented below in Section 7.5. The origin of the "capillary" cavity wave is discussed in detail. Also reported is the splashing behavior of the impinging droplets.

Histories of the centerline sub-cavity liquid film thickness and the liquid film thickness radial distribution are presented in Sections 7.6 and 7.7, respectively. The effects of varying $W e$ and $h_{0}{ }^{*}$ are 
examined for the sub-cavity film thickness histories versus radius. The centerline sub-cavity film thickness was normalized both with the droplet diameter and initial liquid layer thickness.

Sub-cavity volume histories are presented in Section 7.8 and are discussed in detail. The subcavity surface plots have been used to compare with the sub-cavity volume histories. The influence of varying the initial droplet test conditions is discussed. The potential contributions of the subcavity volume results to the MC model are discussed in detail.

\section{Section 7.2: Preliminary Investigation Results and Discussion}

The results in this section are from the preliminary single drop sub-cavity film thickness investigation originally presented in Hillen et al. (2012). The sequence of the droplet impingement evolution spanning from the time of impingement to the collapse of crown is shown in Figure 7.1 for Cases A and B. Splashing was not observed for drop impingements for Case $\mathrm{A}(W e=141, R e=$ 5,700, $\mathrm{Fr}=103$, and $\left.h_{0}{ }^{*}=0.614\right)$ consistent with the splash criteria given in Table 2.2. The crown evolution shown in Figure 7.1 for Case A is relatively stable with no finger formations due to the low momentum, and high viscous and surface tension forces. The cavity is clearly visible for Case A demonstrating the cylindrical shape behavior for drop impingements on thin liquid films. It becomes conical in shape as surface and gravity forces cause it to begin to recede $(t=17.8 \mathrm{~ms})$, consistent with the literature discussed in Section 2.4. Case B $\left(W e=633, \operatorname{Re}=17,100, F r=115\right.$, and $h_{0}{ }^{*}=$ 0.113) had more momentum, and lower viscous and surface tension forces to overcome resulting in

splashing as shown in Figure 7.1. This splashing behavior agrees well with the single drop splashing criteria. The film thickness $\left(h_{0}{ }^{*}=0.113\right)$ utilized for Case B was too thin to visually observe the cavity evolution. 


\section{Case A}

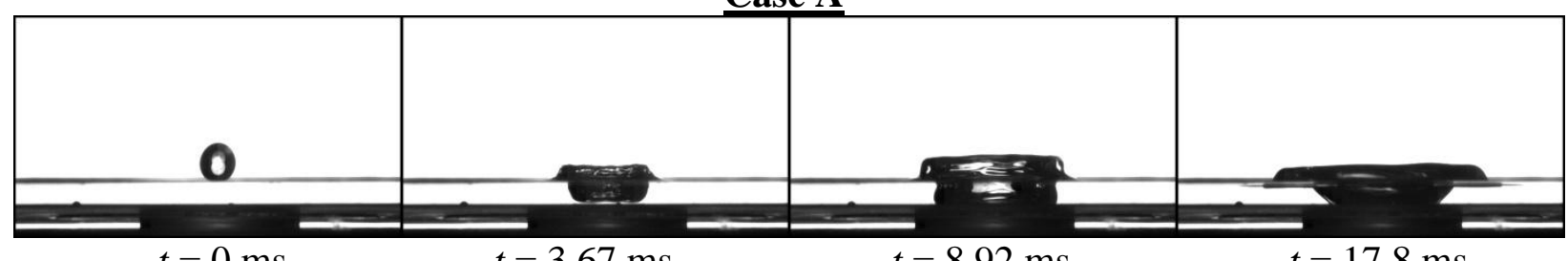

\section{Case B}

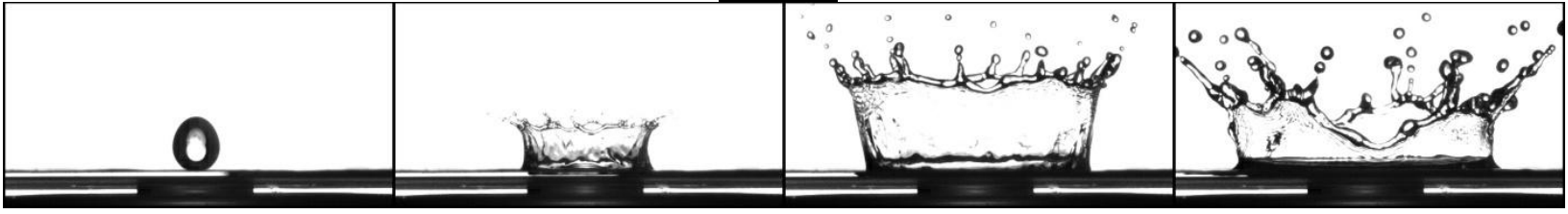

$t=0 \mathrm{~ms}$

$t=3.08 \mathrm{~ms}$

$t=12.9 \mathrm{~ms}$

$t=28.6 \mathrm{~ms}$

Figure 7.1: Sequence of video images for (left to right) initial drop impact, initial crown formation, maximum crown height, and crown collapse for Case A (top) and Case B (bottom) (Hillen et al., 2012)

Figures 7.2 a) and b) show the measured dimensionless bottom crown radius versus dimensionless time for five separate data runs for Cases A and B. The results were computed via image processing as documented in Section 4.3. Bottom crown radii were normalized with the impinging droplet diameter via

$$
R_{B}^{*}=R_{B} / d,
$$

and dimensionless times were computed using Equation 2.8. Bottom crown radii for both Cases A and $\mathrm{B}$ follow the general $t^{0.5}$ crown growth rates from the literature reviewed in Section 2.3.1. Excellent data run-to-run repeatability is observed for the bottom crown radius. Ideally, the bottom crown radius should be 0 at the initial time in Figures 7.2 a) and b), but the desynchronization is due to the lack of measuring capabilities at the point of contact for these preliminary data. Maximum crown radii were observed in Figures $7.2 \mathrm{a}$ ) and b) of nominally $R_{b}{ }^{*}=3.4$ at $\tau \approx 12$, and $R_{b}{ }^{*}=2.5$ at $\tau$ $\approx 11$, respectively. 


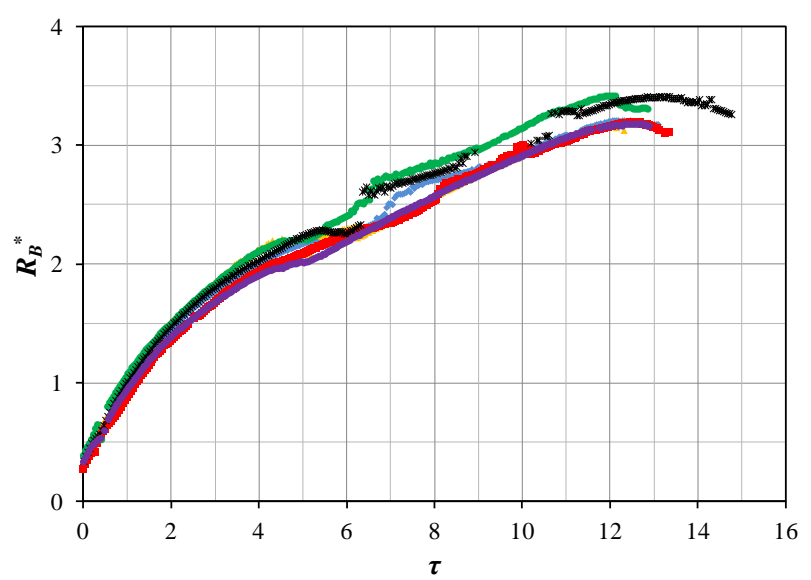

a)

Figure 7.2: Dimensionless bottom crown radius histories for five runs. a) Case A: $W e=141, \operatorname{Re}$ $=5,700, F r=103$, and $h_{0}{ }^{*}=0.614$. b) Case B: $W e=633, \operatorname{Re}=17,100, F r=115$, and $h_{0}{ }^{*}=0.113$ (Hillen et al. 2012).

The dimensionless crown height histories for the respective Case A and B are depicted in Figures 7.3 a) and b). The crown height was also normalized with the initial droplet diameter through the dimensionless expression:

$$
H_{\text {crown }}^{*}=H_{\text {crown }} / d
$$

Maximum crown heights were observed in the respective Figures 7.3 a) and b) are $\sim 0.6 d$ at $4<\tau<5$, and $\sim 1.6 d$ at $\tau \approx 6$. The higher Weber number of the impinging droplet for Case B generates a larger crown with a slightly longer total lifetime than that of Case A, consistent with results from Cossali et al. (2004) covered in Section 2.3. 


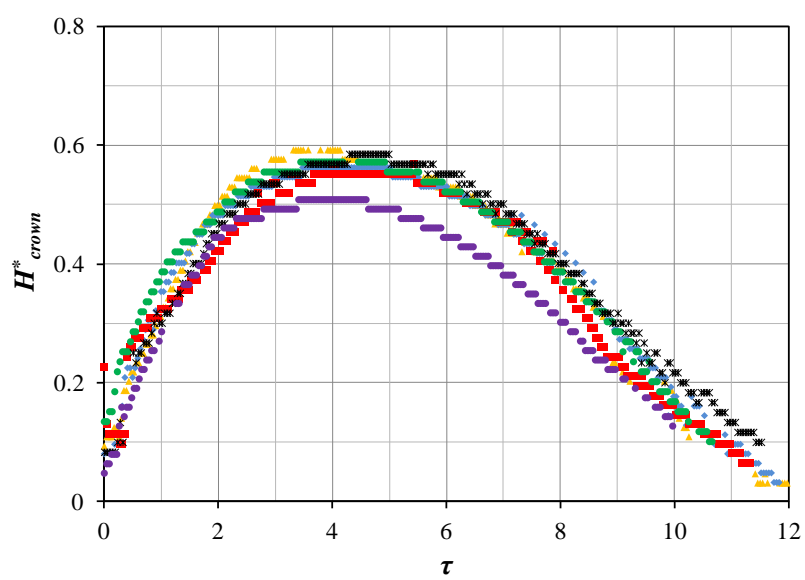

a)

Figure 7.3: Dimensionless crown height histories for five runs. a) Case $A$ : $W e=141, R e=5,700$, $F r=103$, and $h_{0}{ }^{*}=0.614$. b) Case B: $W e=633, \operatorname{Re}=17,100, F r=115$, and $h_{0}{ }^{*}=0.113$ (Hillen et al. 2012).

Centerline sub-cavity liquid film thickness histories for five repeat data runs are shown in the respective Figures 7.4 a) and b). Figure 7.4 a) shows that drop-to-drop liquid film thickness measurements have excellent repeatability for Case A. This repeatability is also generally good in Figure 7.4 b) for Case B. However, some inconsistencies in the minimum liquid film thickness readings due to a subsequent satellite drop are observed. These satellite drops are a product of the primary droplet detachment process from the needle and only observed for droplets with large masses $(d>4 \mathrm{~mm})$. Figure 7.4 shows that a nearly constant minimum sub-cavity film thickness was established during initial moments of drop impingements. This minimum film thickness was maintained until subsequent cavity retraction phase. These observations were consistent with results by Van Hinsberg et al. (2010) discussed in Section 2.4.1. For Case A shown in Figure 7.4 a), an average minimum sub-cavity film thickness of approximately $70 \mu \mathrm{m}$ was observed at $20 \mathrm{msec}$. Both Cases A and B had sub-cavity film thickness histories measured radially outward from the centerline. These film thickness histories also displayed similar excellent repeatability (outer radial measurements for Case B were generally not affected by the satellite droplets). A minimum subcavity film thickness of nominally $81 \mu \mathrm{m}$, or $1.25 \%$ of the initial droplet diameter, was measured for 
the film thickness reading that was not affected by a satellite drop for Case B in Figure $7.4 \mathrm{~b}$ ). This was less than the $2 \%$ minimum liquid sub-cavity film thickness reported by Nikolopoulos et al. (2007) for numerical simulations of a single drop under the same impingement conditions as the current Case B. This was believed to be due to a maximum resolution of two computational cells making up the sub-cavity film thickness in their simulations as previously discussed in Section 2.4. The consistency of the sub-cavity film thickness and crown dimensions data of Cases A and B justified the analysis of only one film thickness history per radial location for the subsequent data presented in Sections $7.5-7.8$.

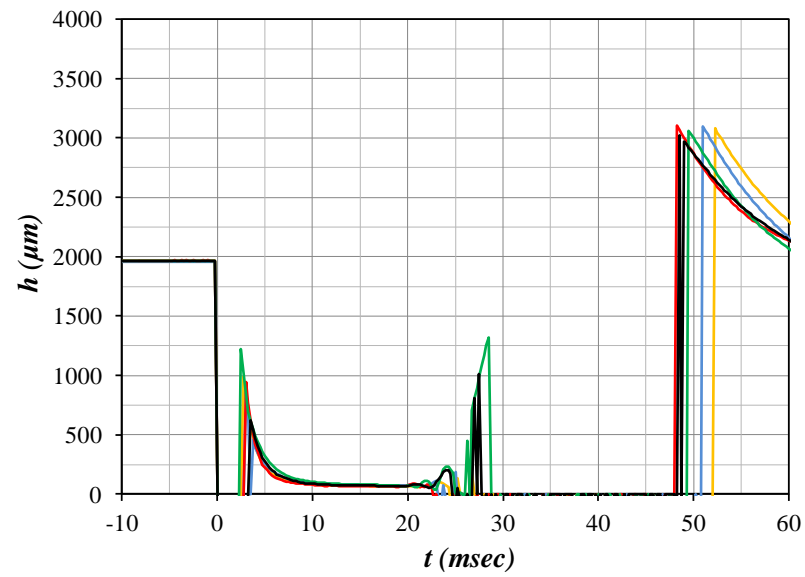

a)

Figure 7.4: Centerline sub-cavity liquid film thickness histories for five runs. a) Case A: $W e=$ 141, $\operatorname{Re}=5,700, F r=103$, and $h_{0}{ }^{*}=0.614$. b) Case B: $W e=633, \operatorname{Re}=17,100, F r=115$, and $h_{0}{ }^{*}=$ 0.113 (Hillen et al. 2012).

A major flaw with utilizing the bottom crown radius to construct the CHR optical probe traverse was discovered in the preliminary investigation. Traversing based on the bottom crown radius resulted in only four relevant sub-cavity liquid film thickness histories for Case A and five for Case B as shown in Figures 7.5 a) and b) respectively. This lack of cavity resolution resulted in reduced accuracy of the sub-cavity volume calculations for these preliminary data. 


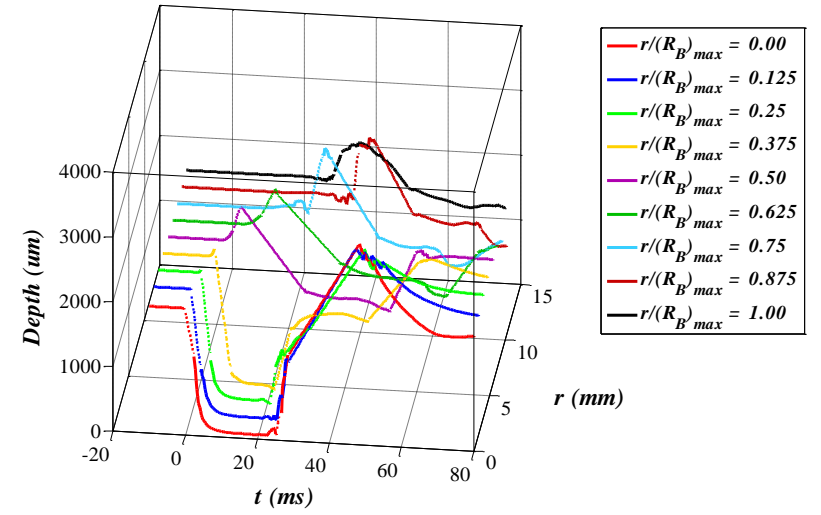

a)

Figure 7.5: Sub-cavity history carpet. a) Case $A: W e=141, \operatorname{Re}=5,700, F r=103$, and $h_{0}{ }^{*}=$ 0.614. b) Case B: $W e=633, \operatorname{Re}=17,100, F r=115$, and $h_{0}{ }^{*}=0.113$ (Hillen et al. 2012).

\section{Section 7.2.1: Experimental and 2D Simulation Comparison}

Companion 2D axisymmetric numerical simulations were also reported in detail in Hillen et al. (2012). Comparisons had been performed with the experimental data to check for consistency to at least perform an initial validation check of one against the other. As depicted in Figure 7.6, the dimensionless bottom crown radius histories for the experiments and simulations are in very good agreement. The small discrepancies between the simulations and experiments may be attributed to image processing not capturing the full bottom crown radius due to the wall meniscus. Crown heights were not available from the simulations for comparison since most of the simulations were 2D. Thus the crown height definition used for the results presented in Figure 4.3 could not be applied. 


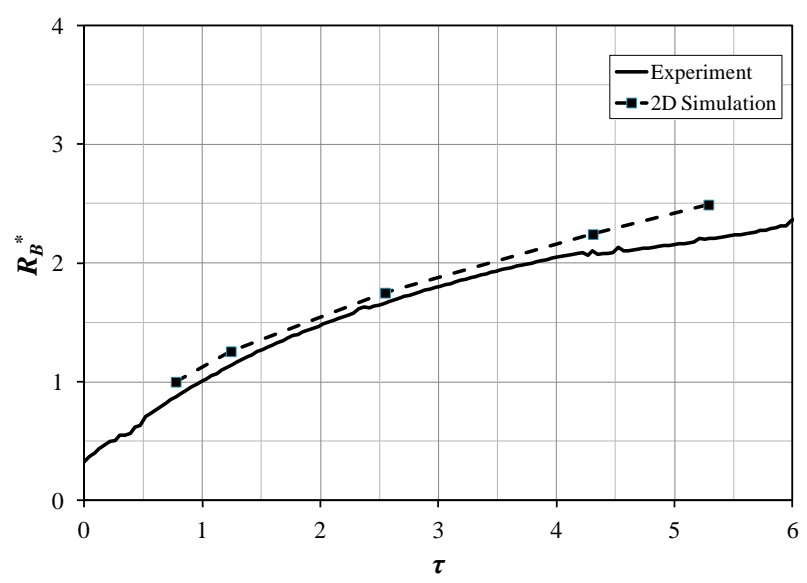

a)

Figure 7.6: Comparison of the bottom crown radius histories from the experiment and 2D simulation. a) Case A: $W e=141, \operatorname{Re}=5,700, F r=103$, and $h_{0}{ }^{*}=0.614$. b) Case B: $W e=633, \operatorname{Re}$ $=17,100, F r=115$, and $h_{0}{ }^{*}=0.113$ (Hillen et al. 2012).

Dimensionless centerline sub-cavity liquid film thickness histories are presented in Figure

7.7 comparing experimental and simulation results. Comparisons between the experiment and simulation results demonstrated excellent agreement for Case A, as shown in Figure 7.7 a). Both results have consistent sub-cavity film thicknesses and similar cavity durations. Figure 7.7 b) also shows good agreement for the sub-cavity film thickness between experiments and simulations of Case B. However, for Case B the simulation cavity begins receding approximately $4 \tau$ to $6 \tau$ sooner than the corresponding experiment, as depicted in Figure 7.7 b). It is unknown as of this time the reason for this discrepancy, but plausible explanations include discrepancies in the initial drop conditions (We, $R e, F r, h_{0}{ }^{*}$, and impact surface properties) between the simulations and experiments, limitations in the 2-D simulations, or unknown limitations with the experimental apparatus. It should be noted that the longer sub-cavity duration at higher Weber numbers are consistent with results by Berberovic et al. (2009) and Van Hinsberg et al. (2010). 


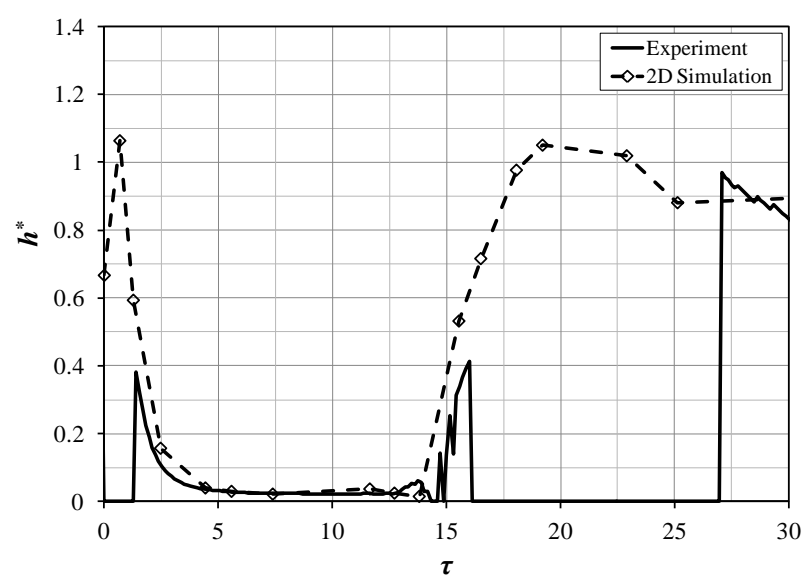

a)

Figure 7.7: Comparison of the centerline sub-cavity liquid film thickness histories from the experiment and 2D simulation. a) Case $A: W e=141, \operatorname{Re}=5,700, F r=103$, and $h_{0}{ }^{*}=0.614$. b) Case B: $W e=633, R e=17,100, F r=115$, and $h_{0}{ }^{*}=0.113$ (Hillen et al. 2012).

\section{Section 7.3: Single drop Sub-cavity Initial Droplet Parameters}

The final dimensionless conditions computed from the Case 1 to Case 5 experiments in terms of $W e, R e, F r$, and $h_{0}{ }^{*}$ are listed in Table 7.1. Also included are the corresponding percent errors with respect to the target results in Table 6.17. Values are computed as an average of the initial drop parameters and film thicknesses for all of the radial profiles for each particular test case. Initial average droplet parameter values and respective standard deviations used to compute the values in Table 7.1 are located in Appendix E. Larger deviations of the We and Re numbers from the target values can be contributed to a lack of control of the environmental temperature. Lab conditions never varied more than $1.75 \%, 1.08 \%$, and $3.72 \%$ for the respective ambient air temperature, absolute atmospheric pressure, and relative humidity during the completion of an individual test case. For consistency, all test cases were completed within the same day they were started. The larger deviations in $W e$ and $R e$ listed in Table 7.1 are a combination of not obtaining the correct impinging droplet parameters and a lack of temperature control in the laboratory. This is especially evident in the Reynolds numbers since the viscosity is highly dependent on temperature. Average 
ambient conditions of the test environment and respective standard deviations are also listed in Appendix E.

Table 7.1: Dimensionless parameters measured in the experiments and their respective percent error to the target values.

\begin{tabular}{|c|c|c|c|c|c|c|c|c|}
\hline & \multicolumn{4}{|c|}{ Experimental Results } & \multicolumn{3}{|c|}{ Percent Error } \\
\hline Case & $h_{0}{ }^{*}$ & $\boldsymbol{h}_{0}^{*}$ & We & $\boldsymbol{R e}$ & $\boldsymbol{F r}$ & $h_{0}{ }^{*}$ & We & $\boldsymbol{R e}$ \\
\hline \multirow{3}{*}{1} & 1.0 & 1.00 & 145 & 1,190 & 100 & $-0.297 \%$ & $-3.44 \%$ & $-1.75 \%$ \\
\hline & 0.5 & 0.50 & 146 & 1,290 & 99.0 & $-0.196 \%$ & $-2.64 \%$ & $6.33 \%$ \\
\hline & 0.2 & 0.24 & 135 & 1,180 & 94.0 & $19.5 \%^{*}$ & $-10.1 \%$ & $-2.82 \%$ \\
\hline \multirow{3}{*}{2} & 1.0 & 1.01 & 410 & 2,250 & 217 & $0.749 \%$ & $2.60 \%$ & $-0.783 \%$ \\
\hline & 0.5 & 0.51 & 413 & 2,270 & 219 & $1.36 \%$ & $3.35 \%$ & $0.0320 \%$ \\
\hline & 0.2 & 0.21 & 414 & 2,290 & 217 & $2.67 \%$ & $3.38 \%$ & $0.903 \%$ \\
\hline \multirow{3}{*}{3} & 1.0 & 1.00 & 622 & 2,670 & 415 & $0.324 \%$ & $3.61 \%$ & $3.99 \%$ \\
\hline & 0.5 & 0.50 & 621 & 2,640 & 415 & $0.454 \%$ & $3.58 \%$ & $2.84 \%$ \\
\hline & 0.2 & 0.19 & 667 & 2,740 & 439 & $-2.54 \%$ & $11.1 \%$ & $6.68 \%$ \\
\hline \multirow{3}{*}{4} & 0.9 & 0.90 & 762 & 3,080 & 398 & $-0.484 \%$ & $-4.81 \%$ & $10.0 \%$ \\
\hline & 0.5 & 0.50 & 771 & 3,090 & 407 & $-0.173 \%$ & $-3.65 \%$ & $10.5 \%$ \\
\hline & 0.2 & 0.20 & 780 & 3,100 & 413 & $0.289 \%$ & $-0.249 \%$ & $10.8 \%$ \\
\hline \multirow{3}{*}{5} & 0.9 & 0.90 & 993 & 3,570 & 510 & $-0.224 \%$ & $-0.670 \%$ & $-7.08 \%$ \\
\hline & 0.5 & 0.50 & 984 & 3,560 & 499 & $-0.988 \%$ & $-1.64 \%$ & $6.78 \%$ \\
\hline & 0.2 & 0.20 & 984 & 3,560 & 407 & $0.583 \%$ & $-1.64 \%$ & $6.53 \%$ \\
\hline
\end{tabular}

Operator error in obtaining the correct initial target liquid layer thickness.

\section{Section 7.4: Sub-cavity Radius Results and Discussion}

Sub-cavity radius measurements were performed for each case listed in Table 6.17 for $h_{0}{ }^{*}=$ 1.0, 0.5, and 0.2 (with $h_{0}{ }^{*}=0.9$ instead of 1.0 for Cases 4 and 5) to determine the radial sub-cavity traverse locations. Sub-cavity radii presented were normalized with the impinging droplet diameter by the following:

$$
R_{c}^{*}=R_{c} / d
$$

Sub-cavity radius histories measured via the two video techniques for varying the $h_{0}{ }^{*}$ and $W e$ are shown in Figures 7.8 and 7.9, respectively. Figure 7.8 shows that the maximum value of the subcavity radius does not change significantly with the variation in $h_{0}{ }^{*}$. However, the lifetimes do significantly increase with increasing $h_{0}{ }^{*}$. Maximum sub-cavity radii of $2.02 d$ at $\tau=18.9,2.10 d$ at $\tau$ $=30.7$, and $2.10 d$ at $\tau=21.3$ have been observed for the respective $h_{0}{ }^{*}=1.0,0.5$, and 0.2 in Figure 
7.8 a). Maximum $R_{c}$ measured from the side-view in Figure $7.8 \mathrm{~b}$ ) for the respective $h_{0}{ }^{*}=1.0,0.5$, and 0.2 are $2.46 d$ at $\tau=27.7,2.43 d$ at $\tau=20.7$, and $2.32 d$ at $\tau=19.0$.

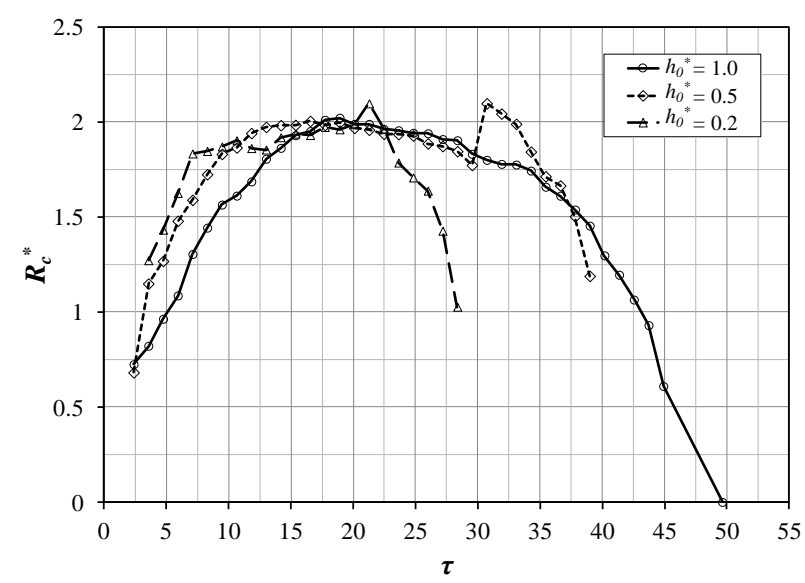

a) a) Bottom-view measurements. b) Side-view measurements.

Figure 7.9 shows that, within the studied parameters, there is a weak correlation between the We and maximum $R_{c}{ }^{*}$. Initially the maximum $R_{c}{ }^{*}$ increases with increasing We until the effect dampens to negligible influence at higher We. For example in Figure 7.9 a maximum $R_{c}{ }^{*}$ for nominally $W e<600$ for the BV results and $W e<400$ for the SV results display a significant, but small dependence on the Weber number. A consistent trend observed for both the BV and SV results was that the sub-cavity radius increases with Weber number more prominently than varying $h_{0}{ }^{*}$. These results are consistent with the literature reviewed in Section 2.4. Also consistent with the literature was the observed initial $R_{c}{ }^{*}$ growth of rate $t^{0.5}$ until the onset of the retraction phase for all scenarios. Similar trends for the influence of the initial drop conditions and initial dimensionless film thickness on the sub-cavity radius lifetime are expected to apply to the sub-cavity volume histories.

There are observed peaks in $R_{c}{ }^{*}$ in most of the data shown in Figures 7.8 and 7.9 during the cavity retraction phase. These peaks are a result of the cavity capillary wave propagating to the impact surface temporally increasing the sub-cavity radius. This was consistent with cavity radius 
histories presented be Berberovic et al. (2009). This propagating cavity capillary wave will be discussed in further detail in the subsequent Section 7.5. Sub-cavity radius histories for the impingement scenarios not shown exhibit similar behavior to those discussed in this section and can be found in Appendix E.
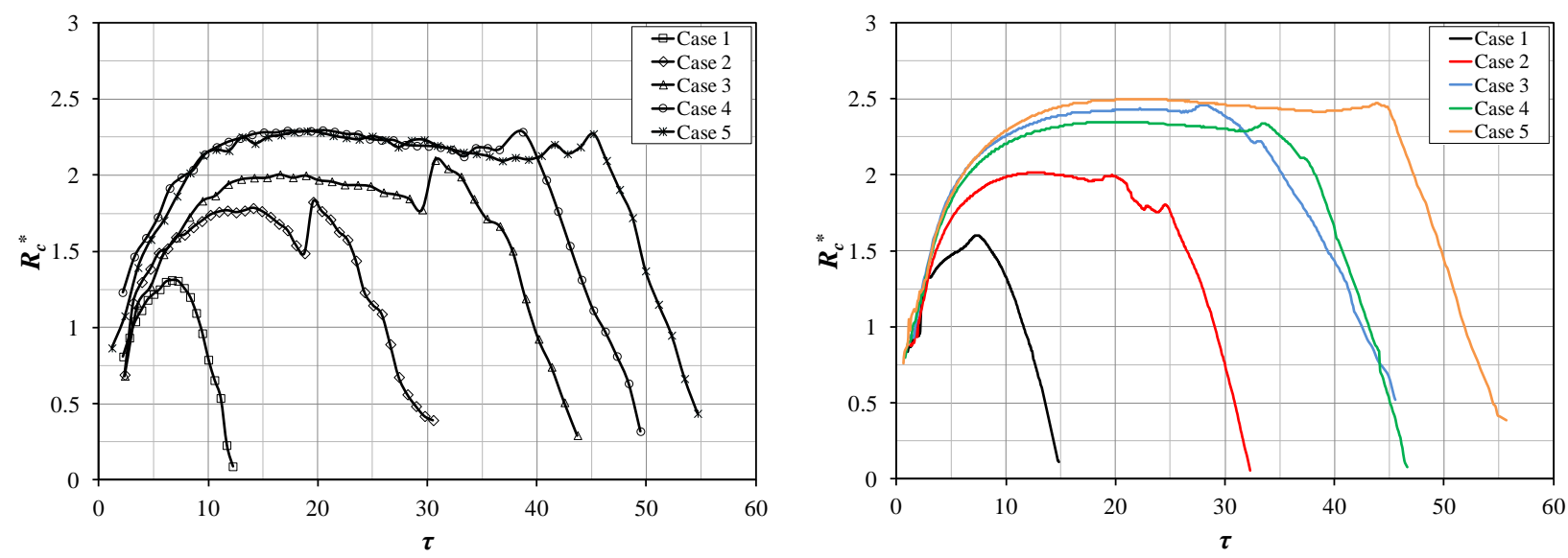

a)

b)

Figure 7.9: Sub-cavity radius histories varying $W e$ at a constant $h_{0}{ }^{*}=0.5$. a) Bottom-view measurements. b) Side-view measurements.

Sub-cavity radii measured via the SV technique resulted in larger measurements than corresponding BV measurements, as shown in Figures 7.8 and 7.9. The coefficient of variance between the maximum $R_{c}{ }^{*}$ measured in Figures $7.8 \mathrm{a}$ ) and b) ranged from 7\% to $14 \%$. It is believed that larger results from the SV method was due to the combination of lack of resolution at the impact surface/bottom cavity interface, and the sub-cavity radius being the average of the last five rows of pixels above the impact surface (see Section 4.4.1). Additional variation in data between the two methods could be contributed to the acrylic mirrors utilized in the BV apparatus. The mirrors tended to slightly warp with use leading to faintly distorted bottom-view cavity videos. Bottom-view subcavity radii were an average of the two major axes, with the typical variance in the measurement ranging from $2 \%$ to $7 \%$ of the average. Figure 7.10 depicts the Case $3, h_{0}{ }^{*}=0.5$ color contour plot of the measured sub-cavity liquid film thickness histories. The film thicknesses have been profiled to 
the largest radial location in which sub-cavity film thicknesses were measured. Superimposed on the contour plot are the analogous BV and SV $R_{c}$ histories including their average. Both the $\mathrm{SV}$ and BV $R_{c}$ match the general sub-cavity film contour (mostly the blue region). Also, it is noted that the bottom cavity $R_{c}$ history under predicts and $\mathrm{SV} R_{c}$ over predicts the actual thin sub-cavity liquid film region. The average of the two $R_{c}$ measurements provided the best fit to the data. These observations are generally applicable for all the impingement scenarios. However results for Cases 4 and 5 show closer convergence of BV and SV sub-cavity radii. Contour plots corresponding to Figure 7.10 for the remaining impingement scenarios may also be found in Appendix E.

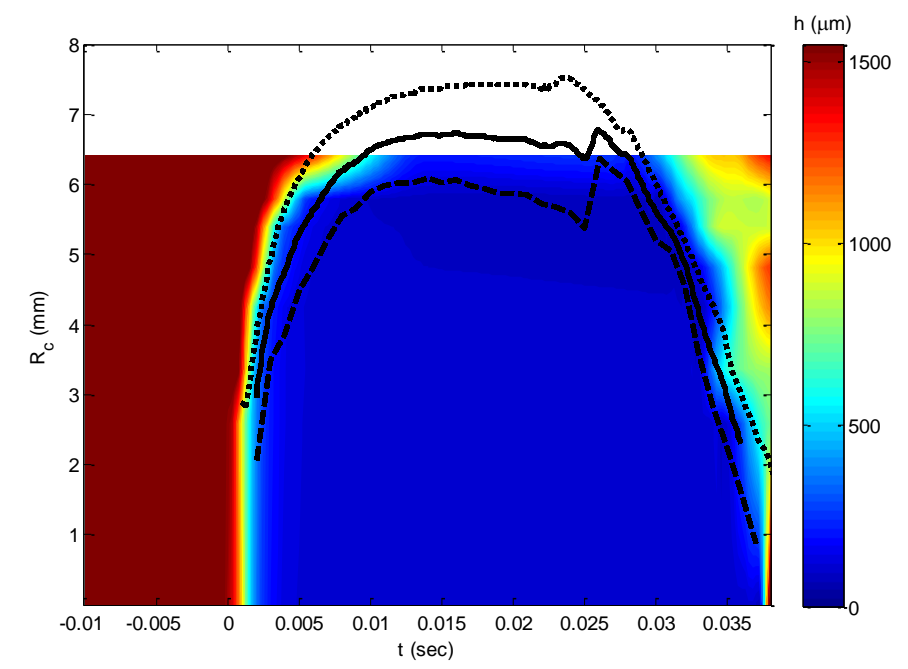

Figure 7.10: Sample contour plot of with the bottom-view (dashed line), side-view (dotted line), and averaged (solid line) sub-cavity radius overlaid for Case $3, h_{0}{ }^{*}=0.5(W e=621, R e=3,090$, $F r=415)$.

\section{Section 7.5: Single Drop Cavity Flow Visualization}

Flow visualization of crown and cavity morphology evolution encompassing the entire test matrix listed in Table 6.17 and full range of $h_{0}{ }^{*}$ was performed. In general the observed cavity and crown behavior follows the same behavior described in literature as discussed in Chapter 2. Since the present work focuses primarily on the cavity behavior, crown behavior discussion herein is limited. A more detailed description of the crown behavior has been presented in Section 2.3. As 
previously discussed in Chapter 2, the crown and cavity are interdependent on each other. This is demonstrated in the sequenced video images of the droplet impingement process for varying the $W e$ and $h_{0}{ }^{*}$ shown in the respective Figures 7.11 and 7.12.

The general cavity trend observed experimentally for all cases consisted of a droplet impinging upon the free surface generating a cavity. The cavity would propagate downward until contacting the impact surface forming a nearly cylindrical shape and creating the sub-cavity liquid film thickness distribution and sub-cavity volume. The cavity then continuously expanded outward along with the crown again illustrating the well established $t^{0.5}$ behavior. During crown collapse the cavity would cease to expand becoming nearly conical in contour before receding and deviating from $t^{0.5}$. These two observations demonstrate the interdependency of the crown and cavity evolution.

Images for drop impingement scenarios shown Figures 7.11 and 7.12 are sequenced to capture the times of the droplet before impact, the cavity during contact with the impact surface, the maximum crown height, and the last stages of crown collapse and cavity retraction. The red horizontal line noted on the images represents the location of the true free surface bottom. Optical distortion was created on the bottom of the free surface due to having aligned the line of sight with the impact surface. The cavity receding phase was always observed to initiate shortly after the crown began to collapse. Consistent with the literature, increasing the Weber number delayed the onset of the receding phase of both the crown and cavity. It also led to an increased crown height as shown in Figure 7.11. In addition, the crown became more susceptible to finger formations and splashing. Higher We had little effect on the initial cavity propagation towards the impact surface. 


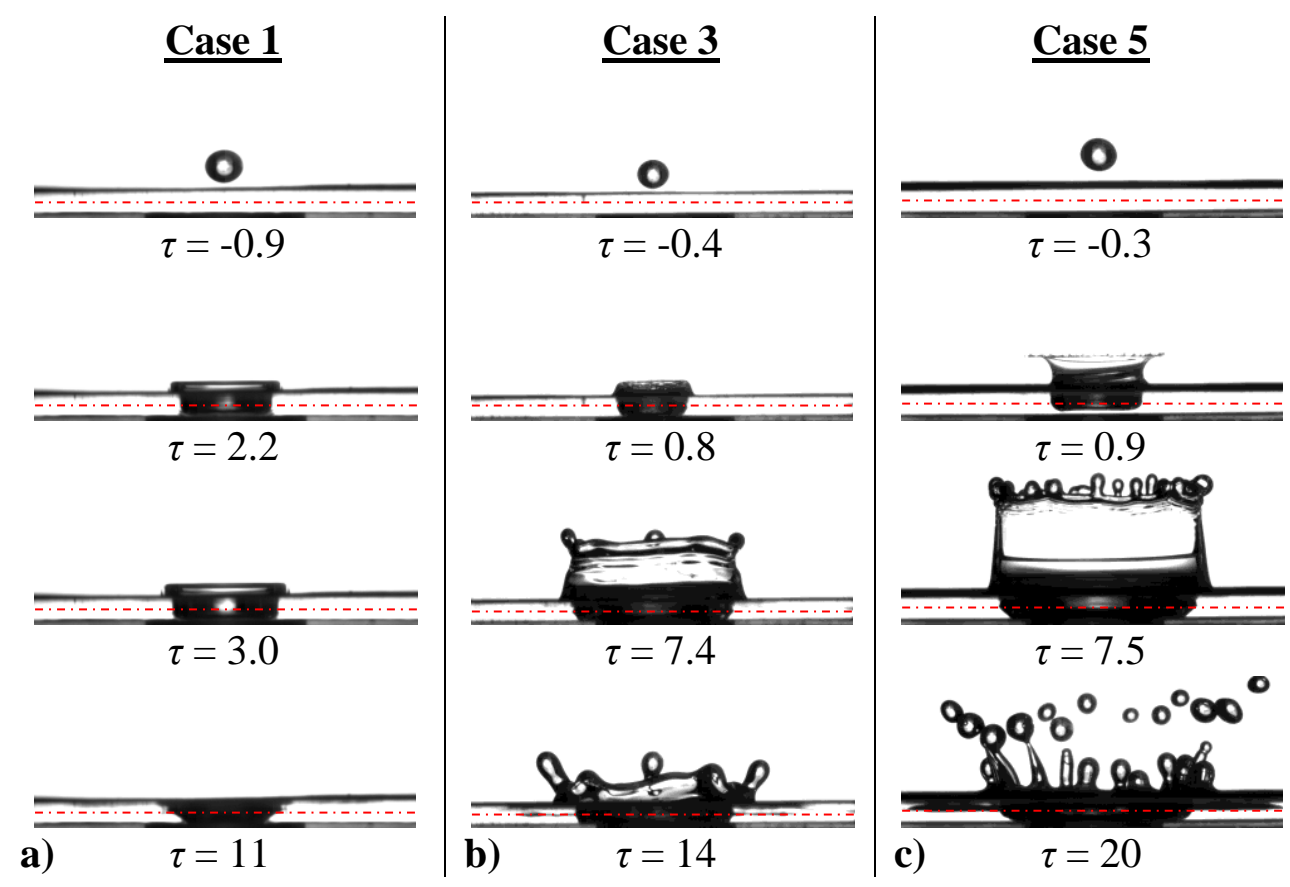

Figure 7.11: Images taken from high-speed videos showing the cavity formation over time at an $h_{0}{ }^{*}=0.5$ for Cases 1,3 , and 5 test conditions. The red dashed line is the true free surface interface. a) $W e=146, \operatorname{Re}=1,290, \mathrm{Fr}=99.0$. b) $W e=621, \mathrm{Re}=\mathbf{2 , 6 4 0 ,} \mathrm{Fr}=415$. c) $W e=984, \mathrm{Re}$ $=3,560, F r=499$ (Hillen et al. 2013).

No significant changes were observed for varying the $h_{0}{ }^{*}$ of Case 1 other than a slightly larger crown height. The corresponding cavity evolution images for $h_{0}{ }^{*}=1.0$ and 0.2 for Case 3 and $h_{0}{ }^{*}=0.9$ and 0.2 for Case 5 are shown in Figure 7.12. As expected, the film thickness had a dramatic influence over the crown formation and splashing. Thinner initial film thickness cases were more susceptible to splashing and produced thinner crowns and larger crown angles. For a given $h_{0}{ }^{*}$ the general cavity shape was not generally affected other than the cylindrical shape occurring earlier for thinner $h_{0}{ }^{*}$. During the initial stages of cavity formation for thicker $h_{0}{ }^{*}$, the cavity generally displayed a more spherical shape consistent with deep pool behavior. These observations were all consistent with the literature reviewed in Chapter 2. Other than the bottom cavity diameter not much on the cavity morphology can be discerned when $h_{0}{ }^{*}=0.2$ in Figures $7.12 \mathrm{~b}$ ) and d) because the thin film lacks visual sharpness. 


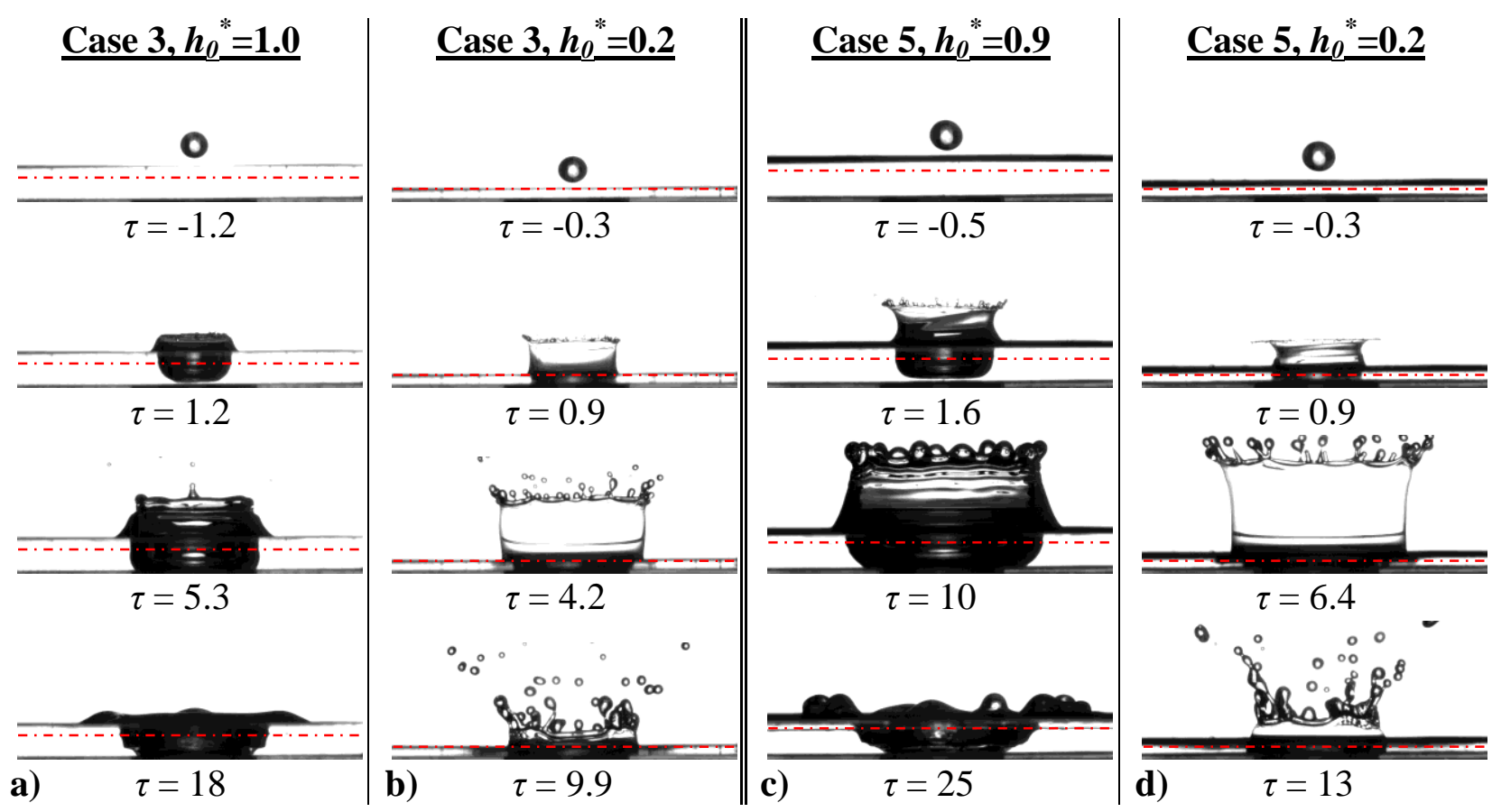

Figure 7.12: High-speed video images showing the crown and cavity formation over time at varying $h_{0}{ }^{*}$ for Cases 3 and 5. The red dashed line is the true free surface interface. a) We $=$ 145, $\operatorname{Re}=1,190, F r=100.0$. b) $W e=135, \operatorname{Re}=1,180, F r=94$. c) $W e=993, \operatorname{Re}=3,570, F r=510$. d) $W e=984, R e=3,560, F r=407$ (Hillen et al., 2013)

During the cavity recession period when the crown is collapsing a noticeable wave on the inside surface of the upper portion of the cavity propagates downward to the impact surface (Berberovic et al., 2009). This wave temporarily expands the sub-cavity radius as it recedes as evident in the results shown in Figures 7.8 and 7.9. The wave has been defined as a capillary wave in the literature (Fedorchenko and Wang, 2004, Roisman et al., 2008, and Berberovic et al., 2009), but is referred to herein as a cavity wave since it is believed to also be driven partially by gravity. A cavity wave is clearly visible at the last time steps in Figures 7.12 a) and c). Figure 7.13 shows a more detailed example of the cavity wave propagation for Case 3 at $h_{0}{ }^{*}=1.0$. The images are sequenced beginning with the maximum crown height before collapse $(\tau=16.0)$, the time immediately before the cavity wave is visible $(\tau=30.7)$, and its propagation to the impact surface $(30.7 \leq \tau \leq 38.5)$. Gravity is believed to be a major contributor to the cavity wave along with surface tension, due to its occurrence coincides with the collapse of the crown mass. It should be noted that 
for the current data the Weber and Froude number are the same order of magnitude while this would not be true for the corresponding Weber number spray droplets. Also, capillary waves generally consist of multiple short wavelength waves, but it was observed experimentally that most of these waves were single events with no more than three occurring at the higher Weber numbers. These two observations support the theory that gravity at least partially drives the termed "capillary" wave (Fedorchenko and Wang, 2004, Roisman et al., 2008, and Berberovic et al., 2009). Cavity wave propagation was observed to occur rapidly with the example contacting the surface in nominally 7.8 dimensionless time units. This propagation agrees well with respective the sub-cavity radius plots in Figure $7.8 \mathrm{~b}$ ) in which the cavity wave is evident for at least $5 \leq \Delta \tau \leq 6$.

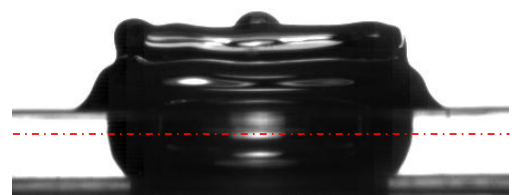

$\tau=16.0$

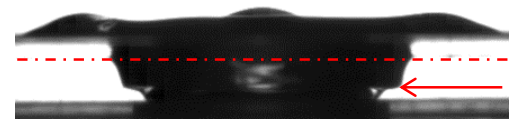

$\tau=36.5$

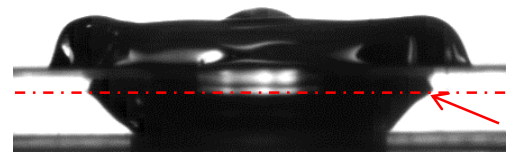

$\tau=30.7$

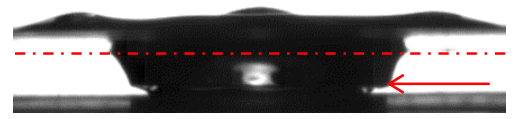

$\tau=37.4$

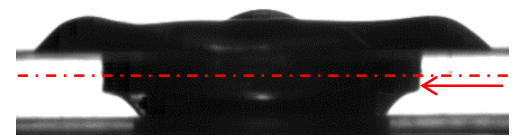

$\tau=34.2$

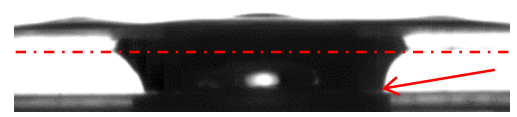

$\tau=38.5$

Figure 7.13: Examination of cavity wave denoted as a capillary wave in literature. Case 3: We $=145, \operatorname{Re}=1,190, F r=100.0$, and $h_{0}{ }^{*}=1.0$. The red dashed line is the true free surface interface and the red arrow highlights the cavity wave.

\section{Section 7.5.1: Additional Splashing Observations}

Splashing behavior has been analyzed for droplet impingement conditions and the results have been presented in Table 7.1. The splashing criteria $K$ and $Y$ have been computed for each test scenario using the respective Equations 2.9 and 2.10 (Cossali et al. 1997, Yarin, 2006). Table 7.2 summarizes the resulting splash criteria and whether consistent splashing was observed from the experiments. To reiterate, splashing would be expected for values of $K>57.1$ for a dry surface and $Y$ 
$>1$ for a thin initial liquid film (see Section Table 2.2). Generally, the $Y$ splashing criteria (Cossali et al. 1997) predicted the splashing outcome.

Table 7.2: Computed splashing limits based on Cossali et al. (1997) compared with experimental observations.

\begin{tabular}{|c|c|c|c|c|}
\hline Case & $\boldsymbol{h}_{\boldsymbol{0}}{ }^{*}$ & $\boldsymbol{K}$ & $\boldsymbol{Y}$ & Observed Splash? \\
\hline \multirow{4}{*}{1} & 1.0 & 911 & 0.114 & No \\
\cline { 2 - 6 } & 0.5 & 944 & 0.221 & No \\
\cline { 2 - 5 } & 0.2 & 855 & 0.319 & No \\
\hline \multirow{4}{*}{2} & 1.0 & 2700 & 0.338 & No \\
\cline { 2 - 5 } & 0.5 & 2720 & 0.637 & No \\
\cline { 2 - 5 } & 0.2 & 2740 & 1.02 & Delayed Splash \\
\hline \multirow{4}{*}{3} & 1.0 & 4040 & 0.506 & No \\
\cline { 2 - 5 } & 0.5 & 4020 & 0.941 & Prompt Splash \\
\cline { 2 - 5 } & 0.2 & 4310 & 1.61 & Prompt and Delayed Splash \\
\hline \multirow{4}{*}{4} & 0.9 & 5020 & 0.701 & Prompt Splash \\
\cline { 2 - 5 } & 0.5 & 5070 & 1.19 & Prompt and Delayed Splash \\
\cline { 2 - 5 } & 0.2 & 5130 & 1.92 & Prompt and Delayed Splash \\
\hline \multirow{3}{*}{5} & 0.9 & 6600 & 0.922 & Prompt Splash \\
\cline { 2 - 5 } & 0.5 & 6530 & 1.53 & Prompt and Delayed Splash \\
\cline { 2 - 5 } & 0.2 & 6530 & 2.44 & Prompt and Delayed Splash \\
\hline
\end{tabular}

An interesting crown formation phenomenon was consistently observed for nearly every test condition for Cases $2-5$ at $h_{0}{ }^{*}=0.2$. An example of this unique crown formation is presented Figure 7.14 for a sequence of images for Case $4(W e=762, \operatorname{Re}=3,080, F r=398)$ at $h_{0}{ }^{*}=0.9$. During the initial droplet impingement an initial crown would form $(\tau=0.265)$. As the droplet continues to penetrate the initial liquid layer a secondary larger crown is formed propagating at a faster speed $(\tau=0.617)$. This new larger crown quickly over takes the slower and smaller initial crown essentially engulfing it $(\tau=1.06)$. However, this initial crown does not immediately merge with the main crown existing on the inside of its rim and continues to propagate upward $(\tau=2.56)$. When the main crown begins to slow as it reaches its maximum height, the initial crown over takes it and completely merges with its $\operatorname{rim}(\tau=7.76)$. This process typically resulted in a prompt splash that 
originates from this initial film as shown in Figure 7.14 at $\tau=7.76$. Prompt splashing due to this behavior was observed to occur on occasion even for Case 2 for $h_{0}{ }^{*}=1$.0. It is also responsible for some the discrepancy between the splashing criteria prediction and the observed predictions in Table 7.2 .

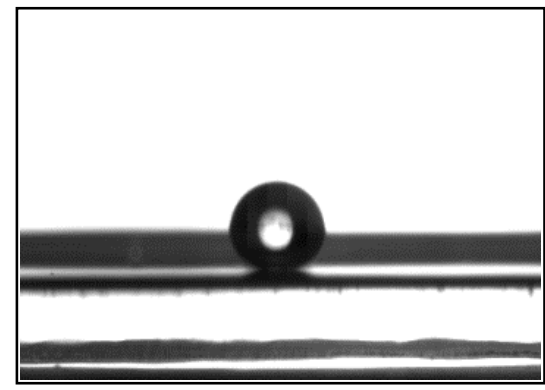

$$
\tau=0
$$

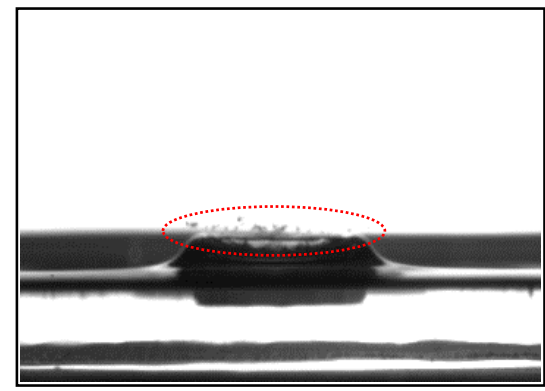

$\tau=1.06$

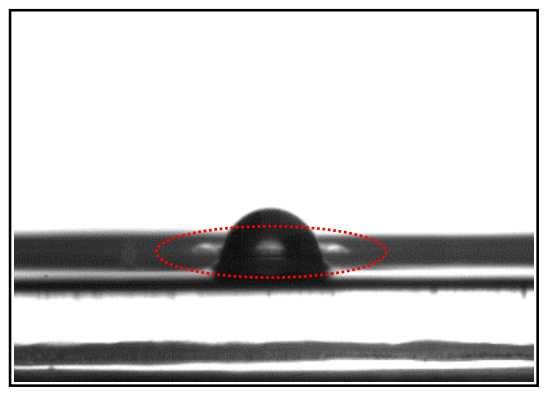

$\tau=0.265$

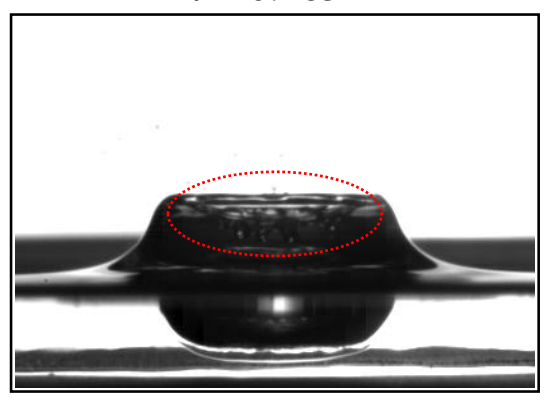

$\tau=2.56$

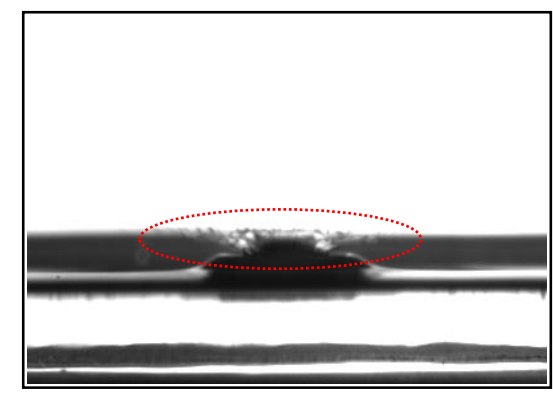

$\tau=0.617$

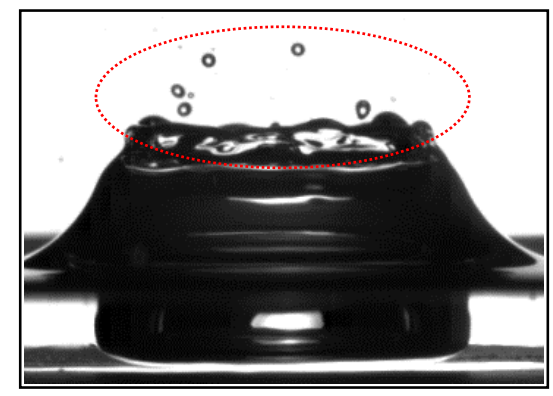

$\tau=7.76$

Figure 7.14: Example of unique prompt splash for Case $4, h_{0}{ }^{*}=0.9(W e=762, R e=3,080, F r=$ 398). The initial splash is highlighted by the dashed ellipse.

\section{Section 7.6: Single Drop Centerline Sub-cavity Film Thickness}

Centerline sub-cavity results presented in this section consist of the improved single drop sub-cavity experiments based on the test plan developed in Section 6.5. The effects of varying the Weber number and initial film thickness on the resulting centerline sub-cavity film thickness histories have been analyzed. Dimensionless sub-cavity film thickness and times were computed via Equations 1.5 and 2.8, respectively. Figure 7.15 shows the measured dimensionless centerline subcavity liquid film thickness histories for the middle Weber number of 640 with $0.2<h_{0}{ }^{*}<1.0$ (Case 3), and for the middle dimensionless film thickness of $h_{0}{ }^{*}=0.5$ for $142<W e<987$ (Cases 1 - 5). It was found that the varying $h_{0}{ }^{*}$ for a constant $W e, R e$, and $F r$ had a negligible effect on minimum sub- 
cavity $h^{*}$ as observed for Case 3 in Figure 7.15 a) with minimum films measured from $\sim 3.2 \%$ to $4.5 \%$ of the initial drop diameter at this Weber number. Earlier initiation versus time of the thin subcavity film thickness was found to occur with decreasing $h_{0}{ }^{*}$ for a specific impact (We, $R e$ ), although this is a minor effect. For an increasing $h_{0}{ }^{*}$ the respective dimensionless nominal times to form the constant minimum $h^{*}$ were $\tau=4.0,4.3$, and 8.0 in Figure 7.15 b). However, $h_{0}{ }^{*}$ has a very pronounced effect on the onset of the cavity collapse, with thinner $h_{0}{ }^{*}$ resulting in shorter cavity durations $\left(\tau=50.0,42.5\right.$, and 27.9 for decreasing $\left.h_{0}{ }^{*}\right)$. The contributions of the dimensionless drop parameters (primarily, variation in We) for a constant $h_{0}{ }^{*}$ on the minimum $h^{*}$ and cavity evolution are shown for $h_{0}{ }^{*}=0.5$ in Figure $7.15 \mathrm{~b}$ ). Increasing the Weber number also is seen to have a negligible effect on the minimum sub-cavity $h^{*}$. Minimum sub-cavity film thicknesses of between $4.1 \%$ to $3.0 \%$ of the initial droplet diameter were measured in Figure $7.15 \mathrm{~b}$ ) as the Weber number is increased. Figure 7.15 b) does demonstrate the cavity duration to be strongly dependent on the Weber number. The cavity collapse time is dramatically increased for higher Weber numbers. Although the Weber number's influence on the total cavity lifetime is comparable to that of $h_{0}{ }^{*}$, it had essentially no influence on the dimensionless cavity formation time. All these observations are consistent with similar previous work as reviewed in Section 2.4. The dependence of the centerline sub-cavity film thickness lifetimes on $W e$ and $h_{0}{ }^{*}$ have been found to be consistent with sub-cavity radius results and flow visualization discussed in the respective Sections 7.4 and 7.5. 


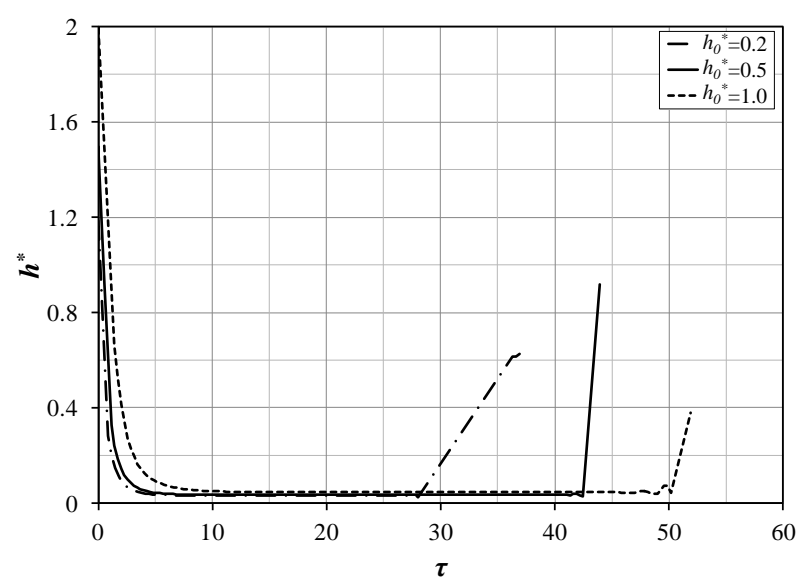

a)

Figure 7.15: The effects of varying the $h_{0}{ }^{*}$ and $W e$ on the centerline dimensionless film thickness. a) Varying $h_{0}{ }^{*}$ for a constant $W e \approx 640, R e \approx 2,700, F r \approx 430$ of Case 3 for. b) Varying the $W e$ at a constant $h_{0}{ }^{*}=0.5$ (Hillen et al., 2013).

The centerline sub-cavity film thickness has also been analyzed as a ratio of the initial film thickness, $\left(h / h_{0}\right)$. Effects of varying the $h_{0}{ }^{*}$ and Weber number on this ratio are shown in Figures 7.16 a) and b) respectively. It was found that for the studied range of conditions, the initial dimensionless film thickness does influence the $h / h_{0}$ ratio at a value of $h_{0}{ }^{*}=0.2$ as shown in Figure 7.16 a). The minimum sub-cavity film thicknesses for this example were measured to nominally increase from $4.6 \%$ to $16 \%$ of the initial film thickness for decreasing $h_{0}{ }^{*}$. Thinner films $\left(h_{0}{ }^{*}=0.2\right)$ are more susceptible to the larger viscous frictional forces in the thin film boundary layer. This results in the sub-cavity film thickness being a higher percentage of the initial layer thickness. It is believed that this particular thin film is comprised mostly of the impinging droplet liquid. As discussed in Section 2.5, numerical simulations tracking the impinging droplet and initial layer fluids were performed in Hillen et al. (2012) for the conditions listed in Table 4.1. Simulation results for a similarly thin initial liquid layer of $h_{0}{ }^{*}=0.113$ for Case B showed the sub-cavity to consist almost entirely of droplet liquid. Additional observations found that Weber number had a negligible influence on $h / h_{0}$ in Figure 7.16 b) consistent with Figure 7.15 b). Minimum sub-cavity film thicknesses between $8.4 \%$ and $6.1 \%$ of the initial liquid film thickness were measured in Figure 7.16 
b) with increasing Weber number. Sub-cavity lifetime behavior was found to be consistent with that observed for Figure 7.15 b).

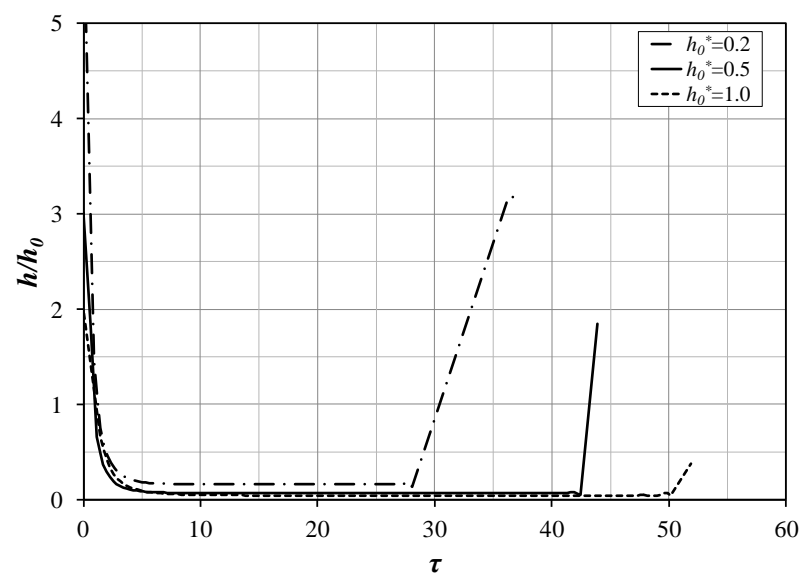

a)

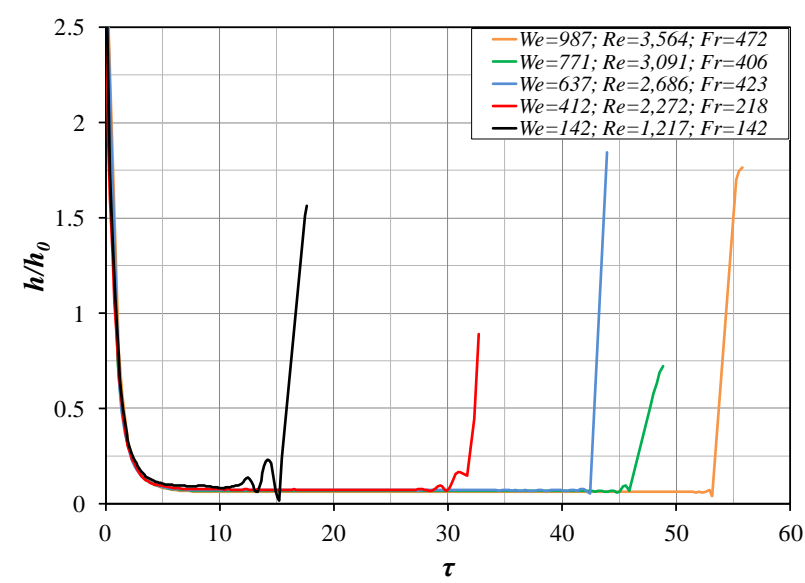

b)

Figure 7.16: The effects of varying the $W e$ and $h_{0}{ }^{*}$ on the centerline film thickness ratio $h / h_{0}$. a) Varying $h_{0}{ }^{*}$ for a constant $W e \approx 640, R e \approx 2,700, F r \approx 430$ of Case 3. b) Varying the $W e$ at a constant $h_{0}{ }^{*}=0.5$ (Hillen et al., 2013).

Trends consistent with those discussed for Figures 7.15 and 7.16 were observed for all Cases at every $h_{0}{ }^{*}$. These additional centerline sub-cavity film thickness history plots are presented in Appendix E.

\section{Section 7.7: Single Drop Cavity Film Thickness Surface Contour Maps}

Cavity liquid film thickness surface contour plots have been constructed from the sub-cavity liquid film thickness versus time and radius. The surface plots are comprised of the film thickness histories using data smoothing and interpolation methods described above in Section 5.4.

The resulting cavity liquid film thickness surface plots are presented below in Figure 7.17 for the entire test matrix given in Table 7.1. A color contour range of $0 \leq h \leq 250 \mu \mathrm{m}$ has been employed to most effectively highlight the sub-cavity contours. The sub-cavity generally displayed a uniform distribution of the liquid film thicknesses. However, a noticeably thinner region of liquid was consistently observed at the outer radial locations for every impingement scenario shown in 
Figure 7.17. These thinner regions occur at the latter stages of the cavity propagation and are expected to influence the sub-cavity volume results. It is thought that the viscous frictional forces in the sub-cavity boundary layer are damping the radial outward momentum of the sub-cavity film as the cavity expands. Increased inertia makes all of the sub-cavity film thicknesses smaller so that the change in the maximum versus minimum $h$ is reduced. Even with the increased drop inertia this thinner region is prominent except for the most extreme drop impingement scenario $\left(\right.$ Case $5, h_{0}{ }^{*}=$ 0.2, We $\approx 987, \operatorname{Re} \approx 3,560$, and $F r \approx 472$ ). This suggests that for the studied cases the Reynolds number (inertial and viscous effects) is the dominant variable in sub-cavity film flow propagation $(R e \leq \sim 2,270)$. Cases 2 - 5 were expected to produce smooth sub-volume histories due to their uniform distribution of the sub-cavity film while Case 1 was expected to provide less clean trends, due to the unevenness of its contours. Examining Case $1(W e \approx 142, \operatorname{Re} \approx 1,220$, and $F r \approx 98.0)$ at $h_{0}{ }^{*}=1.0$ the impinging droplet lacks the momentum for the bulk of the sub-cavity film flow to overcome viscous forces and spread radially outward. Initial film thicknesses of $h_{0}{ }^{*}<1.0$ for Case 1 appear to lead to more uniform sub-cavity liquid film thickness distribution.

Additional investigations of the surface plots depicted in Figure 7.17 show that the maximum cavity radius is nearly constant for a significant portion of the cavity lifetime. The best examples of this occur at the higher $W e$ and $R e$ at $h_{0}{ }^{*}=0.5$. This behavior is fully plausible since it is consistent with the sub-cavity radius measurements presented in Section 7.4.

For the lower Weber number cases and thicker initial liquids films the minimum film thickness histories located at large radius are actually initiated at small times before the minimum film thickness histories near the centerline are established. This phenomenon is due to the "top tail end" of the impinging droplet not fully transitioning into a thin film as the sub-cavity is formed. This was also evident in some of the sub-cavity radius histories in Appendix E due to the image 
processing measuring part of the impinging drop for early times. This resulted in a peak in the data before it transitioned to the smooth $t^{0.5}$ initial sub-cavity radius growth rate.

The cavity wave discussed in the previous Sections 7.4 and 7.5 clearly influenced the surface contour liquid film thickness results. Again referring to Figures 7.8 to 7.10 the peak of the sub-cavity radius due to the cavity wave is typically near the maximum radius that occurs just prior to the onset of the receding phase. It was nearly impossible to capture the transition of the cavity wave contacting the impact surface. This was due to the aforementioned combination of drop-to-drop variability combined with the discrete CHR optical probe traverse method. It is believed that the nearly constant maximum sub-cavity radius is evidence of this cavity wave contacting the surface and delaying the onset of the receding phase. However, measurements of the liquid thickness of the cavity wave propagation via the CHR sensor was obtained for every initial droplet impingement condition of $h_{0}{ }^{*}$ $=0.2$, as shown in Figure 7.17. It occurs near the onset of the receding phase and temporally expands the sub-cavity radius and thus the sub-cavity thin film region. Surface plots for Cases 3 and 5 at $h_{0}{ }^{*}=$ 0.2 clearly show that upon full contact with the impact surface the cavity wave results in the thinnest liquid film region at the outer most radius (the small dark blue region). This occurs at times of nominally 14 to $17.8 \mathrm{msec}$ and 17.3 to $22.5 \mathrm{msec}$ for Cases 3 and 5, respectively. Further examination of the respective nominal time ranges of $12.8<t<14.5 \mathrm{msec}$ and $9.25<t<13.5 \mathrm{msec}$ for Cases 1 and 3 for $h_{0}{ }^{*}=0.2$ shows the transition of the cavity wave contacting the surface. Its thin film is initially evident "behind" the corresponding original film thicknesses at the maximum radial location. Due to these results, it is expected that computed sub-cavity volumes for the impingement scenarios of $h_{0}{ }^{*}=0.2$ will have larger volumes when the cavity wave film thickness merges with the original sub-cavity film region. 

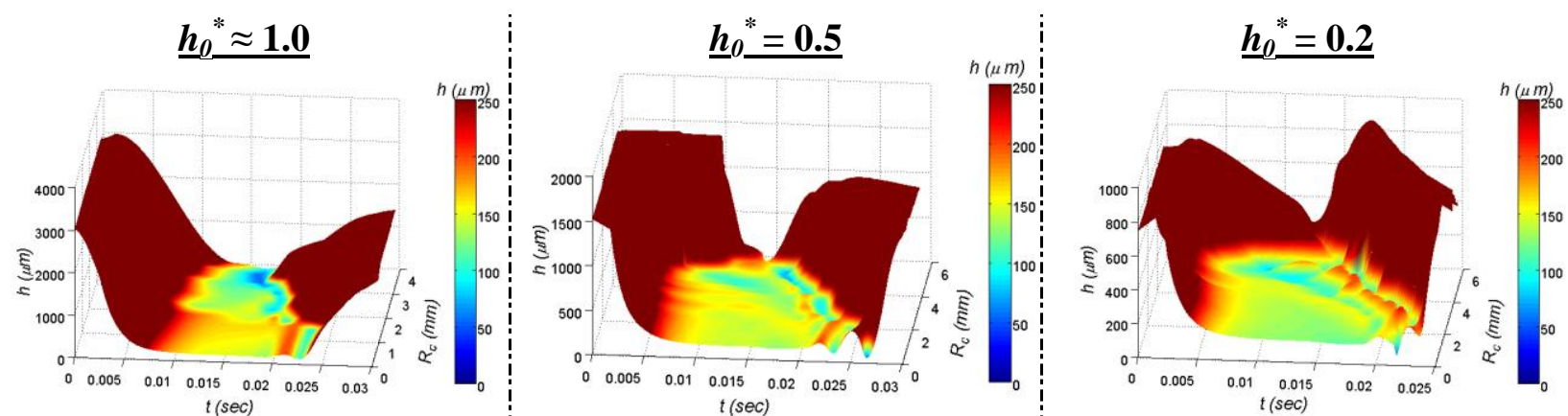

Case 1: $W e=142 ; \operatorname{Re}=1,220 ; F r=98.0$
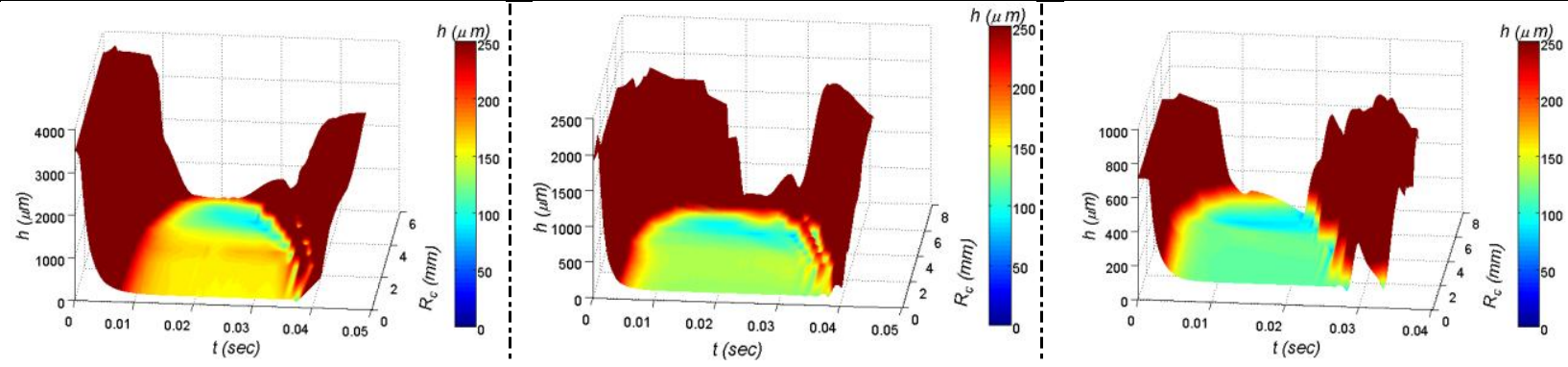

Case 2: $W e=412 ; \operatorname{Re}=2,270 ; \mathrm{Fr}=218$
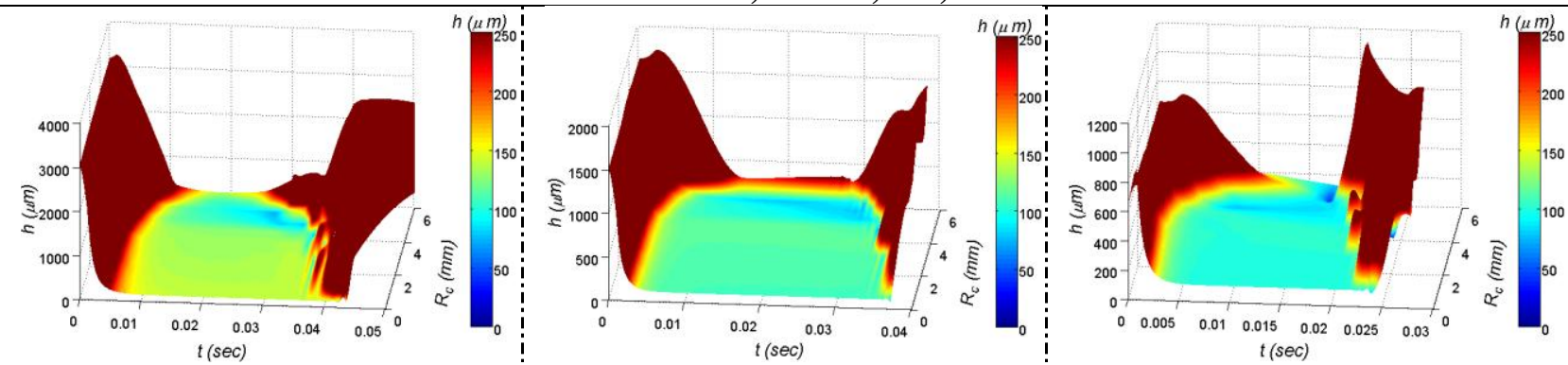

Case 3: $W e=637 ; \operatorname{Re}=2,690 ; F r=423$
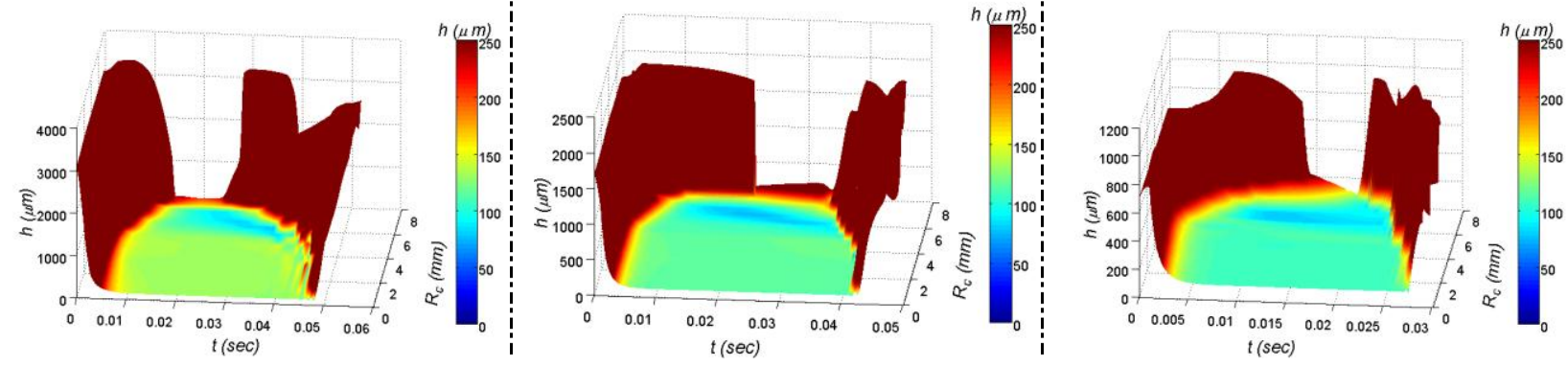

Case 4: $W e=771 ; \operatorname{Re}=3,090 ; \mathrm{Fr}=406$
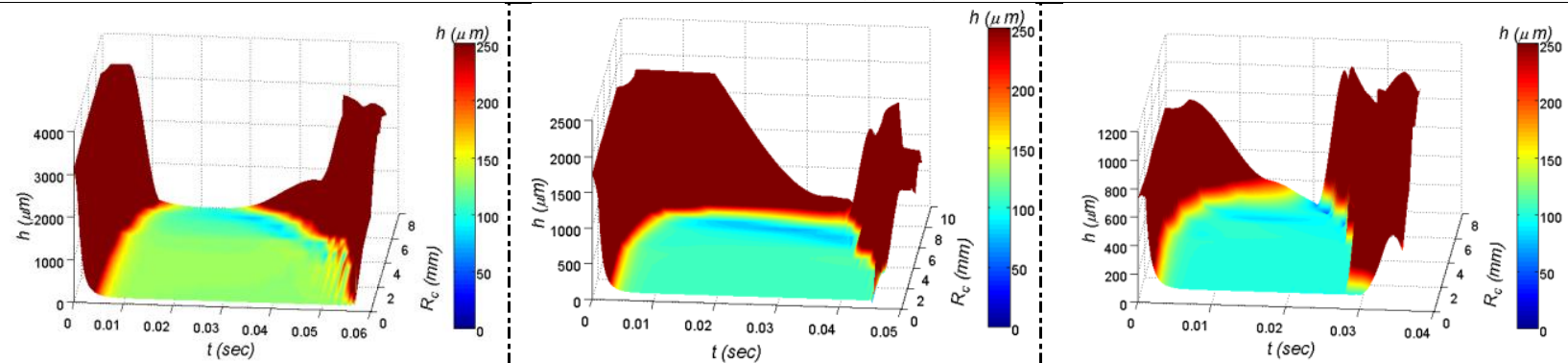

Case 5: $W e=987 ; \operatorname{Re}=3,560 ; \mathrm{Fr}=472$

Figure 7.17: Sub-cavity liquid film thickness surface plots for Cases 1 - 5 at a fixed 0 - $250 \mu m$ contour color scale. Note for cases 4 and $5 h_{0}{ }^{*}=0.9$ in the left hand column. 
A summary of the cavity values are presented in Table 7.3 which have been extracted individually from each Case presented in Figure 7.17. The absolute minimum, absolute maximum, and average sub-cavity liquid film thickness have been computed for the thin sub-cavity region and listed in Table 7.3. The sub-cavity region was defined based on the film thickness constraint limit used to compute sub-cavity volumes (Section 5.5). Therefore, the computed maximum $h$ is similar in thickness to the film thickness constraint limit used for a particular test scenario. They were typically located in the outer radial region where the sub-cavity region begins to transition into the cavity wall. The absolute minimum sub-cavity film thickness was generally consistent between each test scenario and typically located in the thinner sub-cavity regions located radially outward, as previously discussed. However, significantly thinner absolute $h_{\min }$ are listed in Table 7.3 for Cases 1, 3, and 5 for $h_{0}{ }^{*}=0.2$ and Case 1 for $h_{0}{ }^{*}=1.0$. The location and time that these smaller absolute $h_{\min }$ occur corresponds to the time when the cavity wave fully merges with the original sub-cavity liquid film before the onset of retraction. An overall average of the sub-cavity film thickness has also been computed for the sub-cavity region for each test scenario. The $h_{\text {mean }}$ values computed for each test scenario are the same order of magnitude in thickness and follow trends consistent with the centerline sub-cavity film thickness histories. The average sub-cavity film thickness is mostly insensitive to the Weber number, but a noticeable decrease in $h_{\text {mean }}$ with decreasing $h_{0}{ }^{*}$ is observed (excluding Case 1). Table 7.3 also contains the duration of the nearly constant minimum film thickness of the cavity. These times are generally consistent with the trends for varying the We and $h_{0}{ }^{*}$ discussed in the previous Sections 7.3 - 7.6. The maximum sub-cavity radius has also been computed based on the radial sub-cavity film thickness distribution. These results are also listed in Table 7.3 and have trends consistent with the sub-cavity radii measured from the SV and BV 
methods in Section 7.4. It should be noted that for Cases 1,3 and 5 for $h_{0}{ }^{*}=1.0$ the transition of the sub-cavity region to the cavity wall was not captured with the CHR sensor.

Table 7.3: Nominal absolute minimum, absolute maximum, and mean liquid sub-cavity film thickness measured in the sub-cavity region. Also included is the duration of the thin liquid sub-cavity film thickness and maximum sub-cavity radius. Results are for Cases $1-5$ at $h_{0}{ }^{*}=$ 1.0, 0.5, and 0.2.

\begin{tabular}{|c|c|c|c|c|c|c|}
\hline Case & $\boldsymbol{h}_{\boldsymbol{0}}{ }^{*}$ & $h_{\min }(\mu \mathrm{m})$ & $h_{\max }(\mu \mathrm{m})$ & $h_{\text {mean }}(\mu \mathrm{m})$ & $h_{\min }$ Duration (msec) & $R_{c \max }(\mathrm{mm})$ \\
\hline \multirow{3}{*}{1} & 1.0 & 54.4 & 189 & 135 & 13.8 & 3.68 \\
\hline & 0.5 & 66.1 & 200 & 143 & 16.8 & 4.97 \\
\hline & 0.2 & 54.8 & 189 & 138 & 16.5 & 4.82 \\
\hline \multirow{3}{*}{2} & 1.0 & 75.0 & 189 & 145 & 27.8 & 5.80 \\
\hline & 0.5 & 68.5 & 159 & 126 & 31.5 & 6.03 \\
\hline & 0.2 & 70.6 & 145 & 112 & 22.8 & 6.19 \\
\hline \multirow{3}{*}{3} & 1.0 & 71.5 & 170 & 127 & 35.6 & 6.18 \\
\hline & 0.5 & 74.4 & 138 & 106 & 32.1 & 6.10 \\
\hline & 0.2 & 35.8 & 129 & 98.4 & 19.8 & 6.05 \\
\hline \multirow{3}{*}{4} & 0.9 & 71.8 & 170 & 125 & 40.1 & 7.46 \\
\hline & 0.5 & 74.3 & 155 & 110 & 37.5 & 7.25 \\
\hline & 0.2 & 79.3 & 134 & 103 & 23.0 & 7.29 \\
\hline \multirow{3}{*}{5} & 0.9 & 75.8 & 170 & 123 & 50.7 & 7.59 \\
\hline & 0.5 & 73.1 & 145 & 103 & 39.7 & 7.51 \\
\hline & 0.2 & 42.6 & 119 & 98.0 & 26.0 & 7.36 \\
\hline
\end{tabular}

\section{Section 7.8: Sub-cavity Volume Results and Discussion}

Sub-cavity volume histories have been computed for Cases $1-5$ for the three $h_{0}{ }^{*} \approx 1.0,0.5$, and 0.2 through the methodology developed in Chapter 5. The same smoothed and filled sub-cavity liquid film thicknesses versus time and radius used to create the surface plots in Figure 7.17 have been employed in the sub-cavity volume calculations. Presented sub-cavity volumes in this section have been normalized with the volume of the related impinging droplet by:

$$
\mathrm{Vol}^{*}=\mathrm{Vol}_{\mathrm{Vol}} \text { droplet }
$$

Figures 7.18 to 7.20 show the resulting dimensionless sub-cavity volumes including the effects of varying $h_{0}{ }^{*}$ and initial droplet $W e$. For a more in-depth understanding of the presented sub-cavity plots the surface plots, refer to Figure 7.17. 
Examining Figures 7.18 to 7.20 the typical trend observed for $\mathrm{Vol}^{*}$ does not follow the same $t^{0.5}$ growth rate behavior of the crown and cavity radius established in literature and the current results. Instead, the volume follows a time behavior similar to the centerline film thickness as it rapidly reaches a nearly constant plateau that lasts until the onset of the receding phase. One contributing factor to this plateau is that the sub-cavity radius becomes virtually constant during this time until the onset of the receding phase as seen in Figure 7.17. The sharp rise in the sub-cavity volume was not unexpected, since the cavity forms very quickly. Figures 7.15 and 7.16 illustrate that it takes $\tau \approx 4$ - 5 for the rear portion the droplet to reach the impact surface. The thinner regions of liquid film at the outer sub-cavity radius discussed for Figure 7.17 also contribute to the plateau. Furthermore, a slight gradual downward slope in the plateau region is typically found for $h_{0}{ }^{*}>0.2$ for Cases 2 - 5 as shown in Figure 7.18, and Figures 7.19 a) and b). The outer thinner film region is also believed to be the main source of this slight slope. More evidence of the contributions of the propagating cavity wave to the sub-cavity liquid volume can be seen by carefully examining Figure 7.18. The cavity wave causes small sharp dips in the dimensionless volume occur before it plateaus between nominally $5<\tau<15$. The peak occurs at respective approximate dimensionless times of $7.43<\tau<8.68,12.9<\tau<15.3,16.5<\tau<21.2,18.7<\tau<22.7$, and $19.3<\tau<26.4$ for Cases $1-5$ in Figure 7.19 c). All of the sub-cavity volume data shown in Figures 7.18 to 7.20 experienced some oscillation at the times corresponding to the receding phase of the cavity. This was result of the combined effect of the downward inner cavity wave propagation and variations in sub-cavity maps shown in Figure 7.17. A primary cause of the variations in the surface plots was the drop-to-drop variations in the impact process. Surface plots were assembled from $h(t)$ data for different droplet impacts at the differing radii. The resulting assembled waves created what appeared as a "ripple effect" at the final time steps of the sub-cavity surface plots. 
The effects of $h_{0}{ }^{*}$ on the sub-cavity volume for a constant Weber number shown in Figure 7.18 are similar to those described for the centerline film thickness data depicted in Figure 7.15 a). The sub-cavity volume lifetime is equal to the cavity duration. Thus higher initial values of $h_{0}{ }^{*}$ correspond to larger computed dimensionless volumes. This conclusion ignores the sub-cavity volume peaks before the receding phase for $h_{0}{ }^{*}=0.2$ which have a maximum volume nearly the same as the data for $h_{0}{ }^{*}=0.5$ in Figure 7.18, Figures 7.19 a) and b), and Figure 7.20.

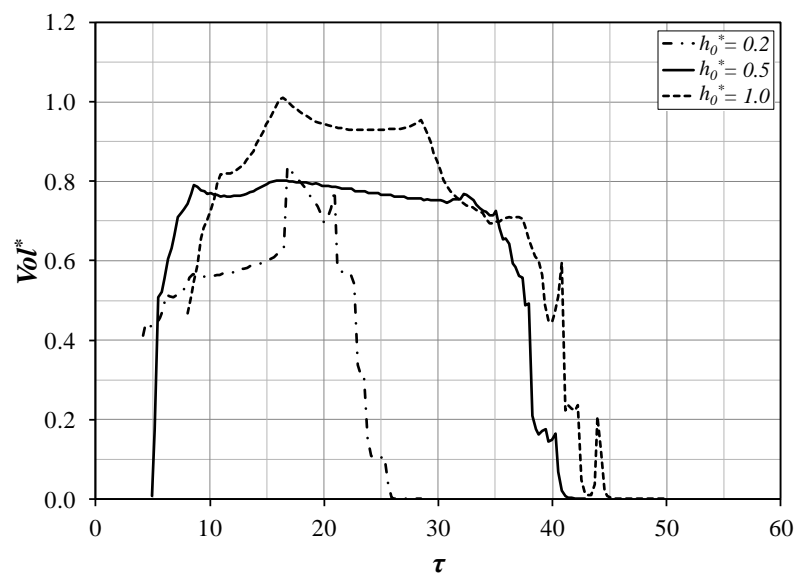

Figure 7.18: Effects of varying the $h_{0}{ }^{*}$ on the dimensionless sub-cavity volume histories at a constant $W e=637, R e=2,686, F r=432$ of Case 3 (Hillen et al., 2013).

Figure 7.19 demonstrates the dimensionless sub-cavity volume dependence on the initial droplet conditions (We, Re, and, $F r$ ). This emphasizes the effects of increased Weber and Reynolds numbers in each of the three plots. The sub-cavity volume lifetimes follow trends similar to those observed for the centerline sub-cavity film thickness profiles in Figure 7.15 a) when Weber number was varied. Consistent with the crown and cavity lifetimes, the sub-cavity volume lifetime increased with an increasing Weber number. This trend was again more prominent than that of increasing the initial film thickness. For a constant $h_{0}{ }^{*}=\sim 1.0$ and 0.5 shown in Figures 7.19 a) and b) it was observed that the maximum value of the sub-cavity volume increased with the Weber number. This influence dampens for the $W e>\sim 412$ for the studied scenarios. A negligible dependence on the 
maximum values of the sub-cavity volume on the Weber number were observed in Figure $7.19 \mathrm{c}$ ) for $h_{0}{ }^{*}=0.2$.
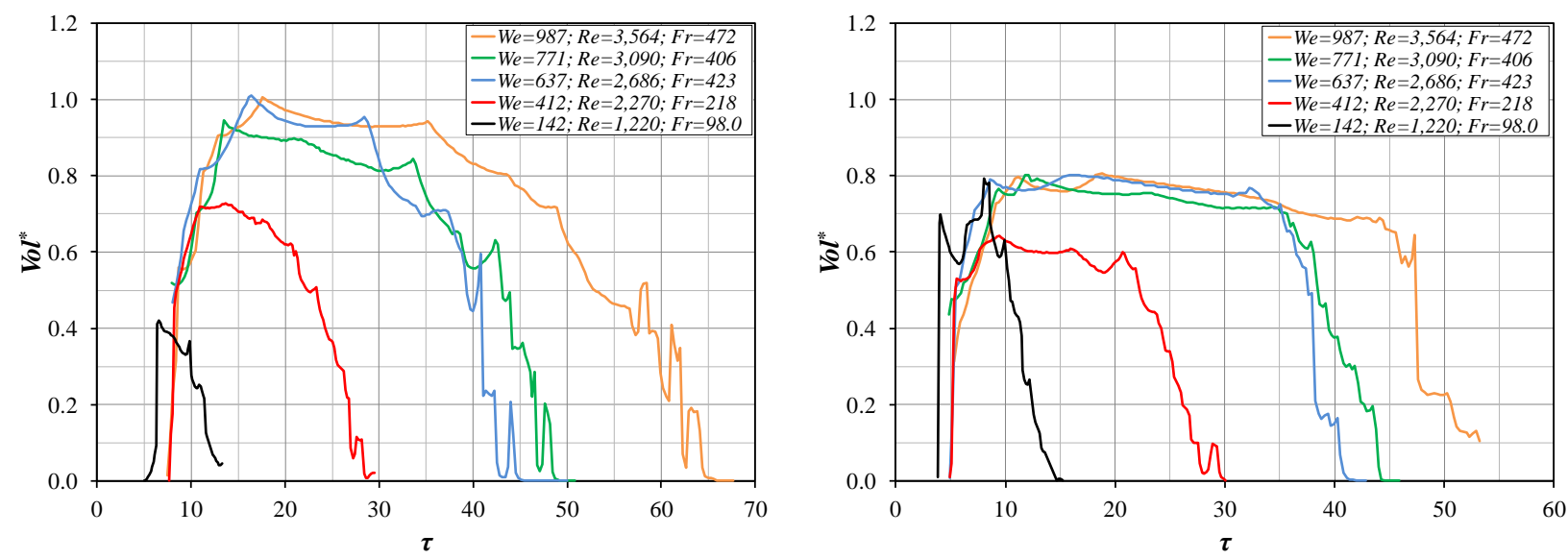

a)

b)

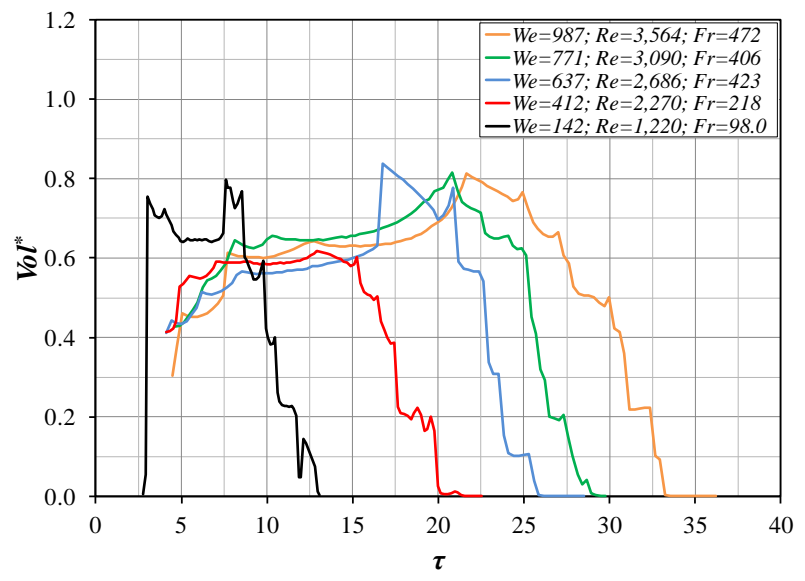

c)

Figure 7.19: Effects of varying $W e$ on the dimensionless sub-cavity volume histories. a) Constant $h_{0}{ }^{*}=1.0\left(h_{0}{ }^{*}=0.9\right.$ for cases 4 and 5). b) Constant $h_{0}{ }^{*}=0.5$. c) Constant $h_{0}{ }^{*}=0.2$.

Sub-cavity volume results presented in Figure 7.20 for Case 1 (lowest Weber number) were not consistent in behavior with the rest of the results. Figure 7.20 show that the smallest sub-cavity volumes were observed for $h_{0}{ }^{*}=1.0$ for a constant $W e$, which was contrary to the previous results shown in Figures 7.18 and 7.19. Also, the sub-cavity volume for Case $1, h_{0}{ }^{*}=1.0$ begins significantly later in time compared to Case 1 results for $h_{0}{ }^{*}=0.5$ and 0.2 . Further Case 1 deviation from the main sub-cavity volume trends can be observed for $h_{0}{ }^{*}=0.5$ and 0.2 results. These two trends are observed to have a very similar duration and sub-cavity volume values. The unique 
behavior of the Case 1 results are due to a lack of initial droplet momentum ( $W e=142, R e=1,220$, and $F r=98.0$ ). The droplet's smaller kinetic energy causes it to be more susceptible to the dampening effects of the layer thickness. Therefore, less potential energy is available to effectively spread the sub-cavity liquid film.

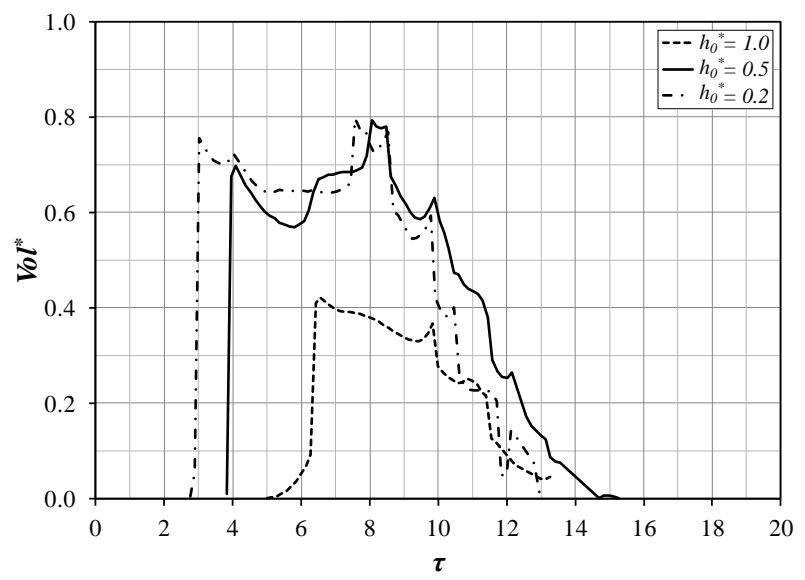

Figure 7.20: Effects of varying the $h_{0}{ }^{*}$ on the dimensionless sub-cavity volume histories at a constant $W e=142, R e=1,220, F r=98.0$ of Case 1 (Hillen et al., 2013). 


\section{CHAPTER 8: CONCLUSIONS AND FUTURE WORK}

\section{Section 8.1: Conclusions}

Sub-cavity volumes created by a single droplet impinging onto a thin initial liquid layer have been successfully computed for a range of dimensionless numbers (We, $R e$, and $\left.h_{0}{ }^{*}\right)$ relevant to a spray of interest. This was accomplished by initially characterizing a full cone water spray generated by a Spraying Systems 1/8-G FullJet nozzle via PDPA for varying flow conditions. A relevant test matrix for the single drop experiments has been created from the integrated global Weber, Reynolds, and Froude numbers utilizing the spray data for the three different operating pressures. Sub-cavity liquid film thicknesses versus time and radius have been measured for each droplet condition in the test matrix utilizing a non-contact chromatic optical thickness sensor and high-speed video imagery. Time dependent sub-cavity volumes have been computed through numerical integration of the subcavity film thickness radial distributions.

PDPA spray results without an impact surface installed displayed consistent trends across the flow conditions. The average droplet diameter has been observed to increase away from the centerline for each nozzle operating pressure. This corresponded to the respective increase and decrease in the spray droplet's average radial and axial velocity. Additional observations showed that the droplet diameter decreased and its average velocities increased with increasing spray nozzle flow condition (gage pressure, and flow rate). The spray data was further examined for $5 \%$ of the diameter population comprised of the largest droplets. The results showed that this diameter range contributed up to nominally $39 \%$ of the total spray mass. Accurate measured volume fluxes were not obtained due to limitations in the PDPA system, along with the very dense spray studied in the present work. This resulted in measured fluxes that deviated significantly from the actual average nozzle flux. 
Additional PDPA studies were performed characterizing the splashed droplets created by the same nozzle spray impinging onto a smooth surface. Data was collected $1.6 \mathrm{~mm}$ above the surface, traversing along the horizontal spray centerline. These measurements were separated into the incoming spray and various splashing droplets. Results showed that the splashed droplets were similar in size to the incoming spray droplets, consistent with Kalantari and Tropea, (2006). These splashed droplets were also relatively slow, never exceeding $3 \mathrm{~m} / \mathrm{s}$, and had low trajectory angles with respect to the surface. This suggests that the spray/splash region may be more complicated than originally had been contemplated based on single drop observations. There may be more interaction between the impinging droplets and incoming spray droplets. Lower quality data was collected at measurement locations above the spray impact surface due to the increased droplet fluxes created by additional splashing droplets. Therefore, splashed droplet diameter and velocities were also measured via PDPA, traversing vertically just outside of the main impinging droplet spray cone. These splashed droplet data have been found to be in consistent agreement with the traverses that were collected above the spray impact surface. Splashing criteria have been computed for the splashed droplets showing that they are not a major contributor to cavity formation in the residual spray film.

A single drop test matrix was created from the studied spray characteristics. This test plan consisted of the following dimensionless number ranges: $150 \leq W e \leq 1,000,1,200 \leq R e \leq 3,300$, and $0.2 \leq h_{0}{ }^{*} \leq 1.0$. A glycerol-water solution was utilized to match the single droplet parameters to the spray data. Due to the size difference of the spray and single droplets, the Froude numbers were unable to be matched for the current apparatus.

Sub-cavity radius histories have been determined for single drop impacts using two complementary high-speed imagery techniques. Good agreement has been observed between the two 
measurements. The behavior of the sub-cavity radius evolution has been shown to be consistent with results by Berberovic et al. (2009). It was found that the maximum sub-cavity radius was relatively insensitive to the initial liquid layer thickness for a given droplet Weber number for the given range of $h_{0}{ }^{*}$ studied in the current work. However, it was observed from the results that the maximum subcavity radius would increase with an increasing Weber number, for Weber numbers below $~ 600$ for the current range. Consistent with literature, increasing the Weber number and/or the initial dimensionless film thickness will delay the onset of the cavity retraction phase (Berberovic et al., 2009 and Hinsberg et al., 2010). The measured sub-cavity radii were in good agreement with the radial distribution of the sub-cavity liquid film thickness.

Single drop high-speed flow visualization has been performed for the single drop impingements of the given test range. A cavity wave was observed in nearly all of the test conditions, which is termed a "capillary wave" in the literature (Fedorchenko and Wang, 2004, Roisman et al., 2008, and Berberovic et al., 2009). However, the high-speed imagery showed evidence that the cavity wave that occurs during the receding phase of the cavity is partially gravity driven. The effects of this cavity wave were observed in the majority of the sub-cavity measurements in the current work $\left(R_{c}{ }^{*}\right.$ data, $h$ surface plots, and $V o l^{*}$ data). Splashing created by the single drop impacts was in good agreement with criteria developed by Cossali et al. (1997).

Single drop sub-cavity liquid film thickness histories have been measured, from the cavity centerline out to the maximum sub-cavity radius, for the test conditions summarized in Table 7.1. Centerline sub-cavity film thickness trends were in good agreement with similar work by Van Hinsberg et al. (2010). During the droplet impingement process a thin, nearly constant minimum sub-cavity liquid film thickness $(\sim 3 \%-4.5 \%$ of the droplet diameter) was quickly established, lasting until the onset of the cavity receding phase. The initial dimensionless film thickness and 
Weber number had only minor effects on the minimum sub-cavity film thickness. However, the lifetime of the cavity increased dramatically with increasing Weber number and/or initial dimensionless layer thickness, consistent with the sub-cavity radius results. Another way to examine the sub-cavity film thickness data was to normalize it with the initial liquid layer thickness. This allowed the effects of viscous friction forces to be observed, where it was observed that $h_{0}{ }^{*}=0.2$ resulted in a thicker $h / h_{0}$ ratio. Surface contour plots of the sub-cavity liquid film thickness showed a nearly uniformly distributed liquid film thickness, except for thinner regions at the outer radial locations. This demonstrates that sub-cavity volumes computed using the current method provide higher quality data compared to using the sub-cavity radius and an average minimum centerline subcavity film thickness. Low Weber numbers of less than approximately 400 displayed uneven subcavity film thickness contours.

Detailed analysis of the sub-cavity volume histories has been performed. Typical sub-cavity volumes resulted in a nearly constant value until the initiation of receding. Deviations from this trend were generally observed for conditions with $h_{0}{ }^{*}=0.2$. For these scenarios the propagating cavity wave would result in a temporary expansion of the radial sub-cavity film thickness distribution. This resulted in significantly larger sub-cavity volumes for this time. Variations in $W e$ and $h_{0}{ }^{*}$ had the same effect on sub-cavity volume lifetimes as were observed for the respective centerline sub-cavity film thickness sub-cavity radii results. That is, the sub-cavity volume lifetime increased with increasing $W e$ and/or $h_{0}{ }^{*}$. It was found that the maximum sub-cavity volume increased with increasing Weber number up to $W e \approx 600$ for $h_{0}{ }^{*}>0.2$. It was also observed that for the given range $h_{0}{ }^{*}$ the sub-cavity volume also increases with thicker initial dimensionless film thicknesses. Plateau regions of the computed sub-cavity volume as percentage of the initial drop volume were nominally $70 \%$ to $90 \%$ for $h_{0}{ }^{*} \approx 1.0,60 \%$ to $75 \%$ for $h_{0}{ }^{*}=0.5$, and $57 \%$ to $65 \% h_{0}{ }^{*}=0.2$. However, sub-cavity 
volumes for $h_{0}{ }^{*}=0.2$ would generally increase to a maximum of nominally $80 \%$ of the initial drop volume when the cavity wave propagated to the floor.

\section{Section 8.2: Future Work}

The presented sub-cavity volume results for the $W e, R e, F r$, and $h_{0}{ }^{*}$ studied herein will be incorporated into the current Monte-Carlo spray cooling model (Kreitzer, 2010) to improve its predictive capabilities. Sub-cavity volumes can be scaled to the spray of interest, and may be treated as a constant over the sub-cavity volume lifetime in the MC model, thus simplifying the computation. Local wall heat fluxes can be computed by applying the localized heat flux enhancement levels computed by Sarkar and Selvam (2009) to the sub-cavity volumes. Including the contributions of these localized cavity heat fluxes is anticipated to improve the accuracy of the MC model.

Also, the contributions of the cavities to the onset of boiling and subsequent $\mathrm{CHF}$ for relevant spray conditions can be investigated. Localized wall heat fluxes that would be needed for the onset of boiling and subsequent dry out in the drop impact cavity can be computed utilizing the measured sub-cavity volumes and their lifetimes. It is hoped that this might lead to an accurate estimate of the overall average heat flux required for onset of $\mathrm{CHF}$.

It is suggested that CFD simulations of the present single droplet test parameters listed in Table 7.1 be performed. Single drop Froude numbers can then be matched to actual spray results to investigate its influence on the sub-cavity region. Also, with the use of CFD the percentage of impinging droplet liquid and initial layer liquid can be computed for Table 7.1. Knowledge of the concentration of the cool droplet liquid in the sub-cavity film would be beneficial. This data would not only improve predictive capabilities of the MC spray cooling model, but also contribute to a greater understanding of the spray cooling heat transfer mechanisms. 
Additional sub-cavity experiments based on the methods developed in the current work should be performed. This includes examining the effects that a single droplet train impinging on a wetted surface would have on the liquid sub-cavity results. It would also be beneficial to investigate the effects of multiple single droplets impinging near the vicinity of each other. In addition, it is suggested that heated sub-cavity single droplet experiments be performed. These data would also contribute to a more accurate MC model.

The PDPA splashed droplet data presented in Chapter 6 can also be incorporated into the initial MC model. If proper volume fluxes can be obtained from this data, it would greatly improve the liquid spray film mass conservation in the MC model. This author recommends that future PDPA be performed for a less dense spray. This would provide smaller drop number fluxes and improve the quality of the PDPA data, ultimately leading to more accurate volume fluxes. 


\section{ACKNOWLEDGEMENTS}

I want to first thank Dr. John Kuhlman for giving me the opportunity to work for him on the current research and for making all of this possible. I am deeply grateful for his unique mentoring and for his invaluable advice throughout my graduate career. Even though at times I loathed him for his extreme attention to detail, it ultimately made me a better individual. When I would feel overwhelmed with a deadline, I would remember that he had an exponentially large work load and still always went out of his way to answer my questions and help me. This motivated me to push myself beyond my known limits and to accomplish my tasks no matter the odds. Most significantly, I want to thank him for providing me with an excellent graduate experience and showing me that you never truly grow up.

I would also like to thank my committee members Dr. Donald Gray and Dr. Gary Morris for going out of their way to provide me with expertise, input, and guidance. I want to specifically thank Dr. Gray for his numerous suggestions and helpful advice. I appreciate that he tolerated reading through my dry and laborious writing style while usually under a deadline. I particularly enjoyed his near limitless historical insights and facts into all things engineering. I would also like to thank Dr. Morris for modifying the droplet generator control box to output a trigger signal. Without it I would not have been able to synchronize my major components. This made my life much easier. He also made time to help us with adjusting the PDPA optics.

I am also grateful for Mr. Chris Menchini for allowing us to utilize his PDPA system and for taking time out of his busy schedule to teach us how to work it. Without him, the current work would be of much lesser quality and a significantly shorter read. I must also thank Dr. Dan Troolin of TSI Inc. for his input and advice on our data and the correct PDPA system settings.

I also thank NASA for providing the funding for my assistantship, my research, and travel. I should also thank the Department of Mechanical and Aerospace Engineering for providing the lab and educational facilities. Specifically, I want to thank the faculty for providing me with a constructive, challenging, and ultimately rewarding learning environment. I also appreciate the staff, especially Debbie Willis, Jean Kopasko, and Marilyn Host, for always answering my many redundant questions about completing my paper work.

I am very thankful for Mr. Cliff Judy who machined the various components of my apparatus. I must acknowledge him for his experience and expertise, and for his various inputs and improvements to my initial designs to make them more practical.

I would be remiss if I did not thank and acknowledge the contributions of my fellow spray cooling project graduate students Mr. Stephen Taylor and Mr. Murat Dinc. Mr. Taylor provided numerous suggestions and ideas that I incorporated into my research. Mr. Dinc spent countless hours performing the necessary related CFD simulations for the current project. More importantly, however, I am most grateful for their friendship.

I am tremendously thankful for my family for without them I would not be the person I am today. Even though they had no idea what my research was about no matter how I tried to explain it, they supported me in furthering my education.

The most important person I must thank is my fiancé Haley Metz. I could not have accomplished any of this without her love and support. She provided me motivation to constantly push myself to be better. During the long dark months of writing my thesis she would bring me food and wait up for me late into the night. 


\section{REFERENCES}

Albrecht, H. E., Damaschke, N., Borys, M., and Tropea, C., "Laser doppler and phase doppler measurement techniques," Springer, Ch. 1-14, 2003.

Bade, K. M. and Schick, R. J., "Phase doppler interferometry volume flux calculation optimization and comparison with nominally point mechanical patternation techniques," $11^{\text {th }}$ Triennial International Annual Conference on Liquid Atomization and Spray Systems, Vail, CO, July, 2009.

Berberovic, E., van Hinsberg, N. P., Jakirlic, S., Roisman, I. V., and Tropea, C., "Drop impact onto a liquid layer of finite thickness: Dynamics of the cavity evolution," Physical Review, The American Physics Society, Vol. 79, pp. 036306-1-9, 2009.

Ching, B., Golay, M. W., and Johnson, T. J., "Droplet impacts upon liquid surfaces," Science 2, Vol. 226, pp. 535-537, 1984.

Cole, V., Mehra, D., Lowry, S., Gray, D., 2005. “A numerical spray impingement model coupled with a free surface film model," Proceedings of the 16th Annual Thermal and Fluids Analysis Workshop (TFAWS-2005), 14 pages. http://tfaws.nasa.gov/TFAWS05/Website/files/ InterdisciplinaryPaperSession/TFAWS05_VCole_ID.pdf

Cossali, G. E., Coghe, A., and Marengo, M., " The impact of a single drop on a wetted surface," Experiments in Fluids, Vol. 22, pp. 462-472, 1997.

Cossali, G. E., Marengo, M., Coghe, A., and Zhdanov, S., "The role of time in single drop splashing thin film," Experiments in Fluids, Vol. 36, pp. 888-900, 2004.

Engel, O. G., "Crater depth in fluid impact," Journal of Applied Physics, Vol. 37, pp. 1798-1808, 1965.

Engel, O. G., "Initial pressure, initial flow velocity, and the time dependence of the crater depth in fluid impacts," Journal of Applied Physics, Vol. 38, pp. 3935-3940, 1967.

Fedorchenko, A. I. and Wang, A. B., "On some common features of drop impact on liquid surfaces," Physics of Fluids, Vol. 165, pp. 1349-1365, 2004.

Gehring, E., Soriano, G., Lin, Y. P., Alvarado, J. L., and Trujillo, M. F., "Film dynamics relevant to spray cooling," 11th International Conference on Advanced Computational Methods and Experimental Measurements in Heat Transfer, July 2010.

Josserand, C. and Zaleski, S., "Droplet splashing on a thin film," Physics of Fluids, Vol. 15, pp.1650-1657, 2003.

Kalantari, D. and Tropea, C., "Spray impact onto flat and rigid walls: Empirical characterization and modeling," International Journal of Multiphase Flow, Vol. 33, pp. 525-544, 2006.

Kalantari, D. and Tropea, C., "Phase Doppler measurements of spray impact onto rigid walls," Experiments in Fluids, Vol. 43, pp. 285-296, 2007.

Kim, J., " Spray cooling heat transfer: The state of the art," Heat and Fluid Flow, Vol. 28, pp. 753767, 2007.

Kreitzer, P. J., "Spray cooling simulation implementing time scale analysis and Monte Carlo method," Ph.D. Dissertation, West Virginia University, Morgantown, WV, 2010.

Kreitzer, P. J. and Kuhlman, J. M., "Visualization of electrohydrodynamic effects and time scale analysis for impinging spray droplets of HFE-7000," in proceedings of the Space Technology and Applications International Forum (STAIF), edited by M. S. El-Genk, AIP Conference Proceedings 969, Melville, New York, pp. 86-93, 2008.

Kuhlman, J. M., Gray, D. D., and Kreitzer P. J., "Progress in modeling of spray cooling," Proceedings of the $24^{\text {th }}$ European Conference on Liquid Atomization and Spray Systems 
(ILASS), 7 pages, Estoril, Portugal, September 5-6, 2011. Poster presented at the European Conference on Liquid Atomization and Spray Systems (ILASS), Estoril, Portugal, September 5-7, 2011.

Kuhlman, J.M., Kreitzer, P. J., Mehra, D., Gray, D.D., and Yerkes, K.L., "Influence of the Coulomb Force on Spray Cooling," STAIF ${ }^{11}$ Conference on Thermophysics Applications in Microgravity, edited by M. El-Genk, AIP Conference Proceedings 504, New York, 2007.

Kunkel, M. and J. Schulze, J., "Noncontact measurement of central lens thickness," Glass Science Technology, Vol. 78, pp. 245-247, 2005.

Hillen, N. L., Kuhlman, J. M., Dinc, M., and Gray, D. D., "Drop Impingement on Wet and Dry Surfaces," Paper AIAA-2012-2960, AIAA 42nd Fluid Dynamics Conference, New Orleans, LA, June 25-28, 2012.

Hillen, N. L. and Kuhlman, J. M., "Characterization of sprays onto an unheated surface for spray cooling applications," $38^{\text {th }}$ AIAA Dayton-Cincinnati Aerospace Science Symposium (DCASS), Dayton, OH, March 6, 2013a.

Hillen, N. L. and Kuhlman, J. M., "Single impinging droplets on a preexisting liquid surface using high-speed video visualization techniques for bottom cavity size," $88^{\text {th }}$ Annual Meeting of the West Virginia Academy of Science, Davis, WV, April 6, $2013 \mathrm{~b}$.

Hillen, N. L, Menchini, C. P., Morris, G., Dinc, M., Taylor, J. S., Gray, D. D., and Kuhlman, J. M., "Droplet impact time histories for a range of Weber numbers and liquid film thicknesses for spray cooling application,” Paper AIAA-2013-2976, AIAA 43rd Fluid Dynamics Conference, San Diego, CA, June 24-27, 2013.

Horacek, B., Kiger, K. T., and Kim, J., "Single nozzle spray cooling heat transfer mechanisms," International Journal of Heat and Mass Transfer, Vol. 48, pp. 1425-1238, 2005.

Lin, L. and Ponnappan, R., "Heat transfer characteristics of spray cooling in a closed loop," International Journal of Heat and Mass Transfer, Vol. 46, pp. 3737-3746, 2003.

Lee, M. M. and Hanratty, T. J., "The inhibition of droplet deposition by the presence of a liquid film," International Journal of Multiphase Flow, Vol. 14, pp. 129-140, 1988.

Macklin, W. C. and Metaxes, G. J., "Splashing of drops on liquid layers," Journal of Applied Physics, Vol. 47, pp. 3963-3970, 1976.

Macklin, W. C. and Hobbs, P. V., "Subsurface phenomena and splashing of drops on shallow liquids," Journal Storage (JSTOR), Vol. 166, pp. 107-108, 1969.

Manzello, S. L. and Yang, J. C., “An experimental study of a water droplet impinging on a liquid surface," Experiments in Fluids, Vol. 32, pp. 580-589, 2002.

Mathews, W. S., Lee, C. F., and Peters, J. E., "Experimental investigations of spray/wall impingement," Atomization and Sprays, Vol. 13, pp. 223-242, 2003.

Menchini, C. P., "Experimental flow characterization and computational model development of aqueous film forming-foam (AFFF) jets," Ph.D. Dissertation, West Virginia University, Morgantown, WV, 2013.

Metropolis, N. and Ulam, S, "The Monte Carlo Method," Journal of the American Statistical Association, Vol. 44, No. 247, pp. 335-341, 1949.

Mundo, C. H. R., Sommerfeld, M., and Tropea, C., "Droplet-wall collisions: Experimental studies of the deformation and breakup process," International Journal of Multiphase Flow, Vol. 21, pp. 151-173, 1995.

Nikolopoulos, N., Theodorakakos, A., and Bergeles, G., "Normal impingement of a droplet onto a wall film: numerical investigation," International Journal of Heat and Fluid Flow, Vol. 26, pp. 119-132, 2005. 
Nikolopoulos, N., Theodorakakos, A., and Bergeles, G., "Three-dimensional numerical investigation of a droplet impinging normally onto a wall film," Journal of Computational Physics, Vol. 225, pp. 322-342, 2007.

Okawa, T. Shiraishi, T., and Mori Toshiaki, "Effect of impingement angle on the outcome of a single drop impact onto a plane water surface," Experiments in Fluids, Vol. 44, pp. 331-339, 2007.

Pautsch, A. G., Shedd, T. A., and Nellis, G. F., "Thickness measurements of the thin film in spray evaporative cooling," Paper 0-7803-8357-5, $9^{\text {th }}$ Inter Society Conference on Thermal Phenomena, June 1-4, 2004.

Pautsch, A. G. and Shedd, T. A., "Adiabatic and diabatic measurements of the liquid film thickness during spray cooling with FC-72," International Journal of Heat and Mass Transfer, Vol. 49, pp. 2600-2618, 2006.

Rein, M., "Phenomena of liquid drop impact on solid and liquid surfaces," Fluid Dynamics Research, Vol. 12, pp. 61-93, 1993.

Rioboo, R. , Bauthier, C., Conti, J., Voue, M., and De Coninck, J., “ Experimental investigation of splash and crown formation during single drop impact on wetted surfaces," Experiments in Fluids, Vol. 35, pp. 648-652, 2003.

Rioboo, R., Marengo, M., Cossali, G. E., and Tropea, C., "Comparison of drop impact: dry and wetted cases," ILASS-Europe, pp.11.1-5, 2000.

Roisman, T., Tropea, C., and Stephan, P., " Dynamics of the cavity and surface film for impingements of single drops on liquid films of various thicknesses," Journal of Colloid and Interface Science, Vol. 350, pp. 336-343, 2010.

Roisman, I. V., van Hinsberg, N. P., and Tropea, C., " Propagation of kinematic instability in liquid layer: Capillary and Gravity effects," Physical Review, The American Physical Society, Vol. 77, pp. 046305-1-7, 2008.

Santini, M., Fest-Santini, S., Cossali, G. E., and Guilizzoni, M., "Drop impact onto a deep pool: study of the crater evolution," $24^{\text {th }}$ European Conference on Liquid Atomization and Spray Systems (ILASS), Estoril, Portugal, September 5-6, 2011.

Sarkar, S. and Selvam, R. P., "Direct Numerical Simulation of Heat Transfer in Spray Cooling Through 3D Multiphase Flow Modeling Using Parallel Computing," Journal of Heat Transfer, ASME, Vol. 131, pp. 121007-1 to 121007-8, 2009.

Schwarzkopf, J. D., Shakal, J. S., and Bonuccelli, C., "A new method for minimizing volumetric flux error associated with PDPA measurements in the dilute region of the full cone pressure swirl atomizers," Paper ICLASS06-163, International Conference on Liquid Atomization and Spray Systems (ICLASS), Kyoto, Japan, Aug. 27-Sept. 1, 2006.

Shedd, T. A. and Pautsch, A. G., "Spray impingement cooling with single- and multiple-nozzle arrays. Part II: Visualization and empirical models," International Journal of Heat and Mass Transfer, Vol. 48, pp. 3176-3184, 2005.

Shin, J. and McMahon, T. A., “The tuning of a splash,” Physics of Fluids, Vol. 8, pp. 1312-1317, 1990.

Silk, E. A., Golliher, E. L., and Selvam, R. P., "Spray cooling heat transfer: Technology overview and assessment of future challenges for micro-gravity application," Energy Conversion and Management, Vol. 49, pp. 452-468, 2008.

Sivakumar, D. and Tropea, C., "Splashing impact of a spray onto a liquid film," Physics of Fluids, Vol. 14, pp. L85-L88, 2002. 
Soriano, G., Lin, Y. P., and Alvarado, J. L., "Experimental Characterization of Single and Multiple Droplet Impingement on Surfaces Subject to Constant Heat Flux Conditions," Paper IHTC14-22217, Proceedings of the 14th International Heat Transfer Conference ITHC14, Washington, DC, August 8-13 2010.

Tilton, D. E., "Spray Cooling,” Ph.D. Dissertation, University of Kentucky, Lexington, KY, 1989. TSI Inc., LDV/PDPA System Installation Manual. February 2005.

Dr. Dan Troolin, TSI, Inc, Personal Communication, 2012.

Van Hinsberg, N. P., Budakli, M., Göhler, S., Berberovic, E., Roisman, I. V., Gambaryan-Roisman, T., Tropea, C., and Stephan, P., "Dynamics of the cavity and the surface film for impingements of single drops on liquid films of various thicknesses," Journal of Colloid and Interface Science, Vol. 350, pp. 336-343, 2010.

Vander Wal, R. L., Berger, G. M., and Mozes, S. D., "Droplets splashing upon films of the same fluid of various depths," Experiments in Fluids, Vol. 40, pp. 33-55, 2006.

Wang, A. and Chen, C. C., "Splashing of a single drop onto very thin liquid films," Physics of Fluids, Vol. 12, pp. 2155-2158, 2000.

Wang, M. and Watkins, A. P., "Numerical modeling of Diesel spray impaction phenomena," International Journal of Heat and Fluid Flow, Vol. 14, pp. 301-311, 1993.

Widmann, J. F., Presser, C., and Leigh, S. D., "Improving phase Doppler volume flux measurements in low data rate applications," Measurement Science and Technology, Vol. 12, pp. 11801190, 2001.

Winkler, C. M. and Peters, J. E., "Refrigerant droplet size measurements in conjunction with a novel method for improving flow distribution in evaporators," Aerosol Science and Technology, Vol. 36, pp. 734-741, 2002.

Xu, L., Zhang, W. W., and Nagel, S. R., "Drop splashing on a dry smooth surface," Physical Review Letters, Vol. 94, pp. 1-4, 2005.

Yarin, A. L., "Drop impact dynamics: splashing, spreading, receding, bouncing...," Annual Review Fluid Mechanics, Vol. 38, pp. 159-192, 2006.

Yerkes, K.L., Michalak, T., Baysinger, K., Puterbaugh, R., Thomas, S.K., and McQuillen, J., "Variable-gravity effects on a single-phase partially-confined spray cooling system," Paper AIAA-2006-0596, 44 ${ }^{\text {th }}$ AIAA Aerospace Sciences Meeting and Exhibit, Reno NV, 9-12 Jan. 2006. 


\section{Appendix A: $\quad$ CAD DraWINGS}

This Appendix contains the CAD drawings with dimensions of the major components of the single drop apparatus. Units are in inches. 


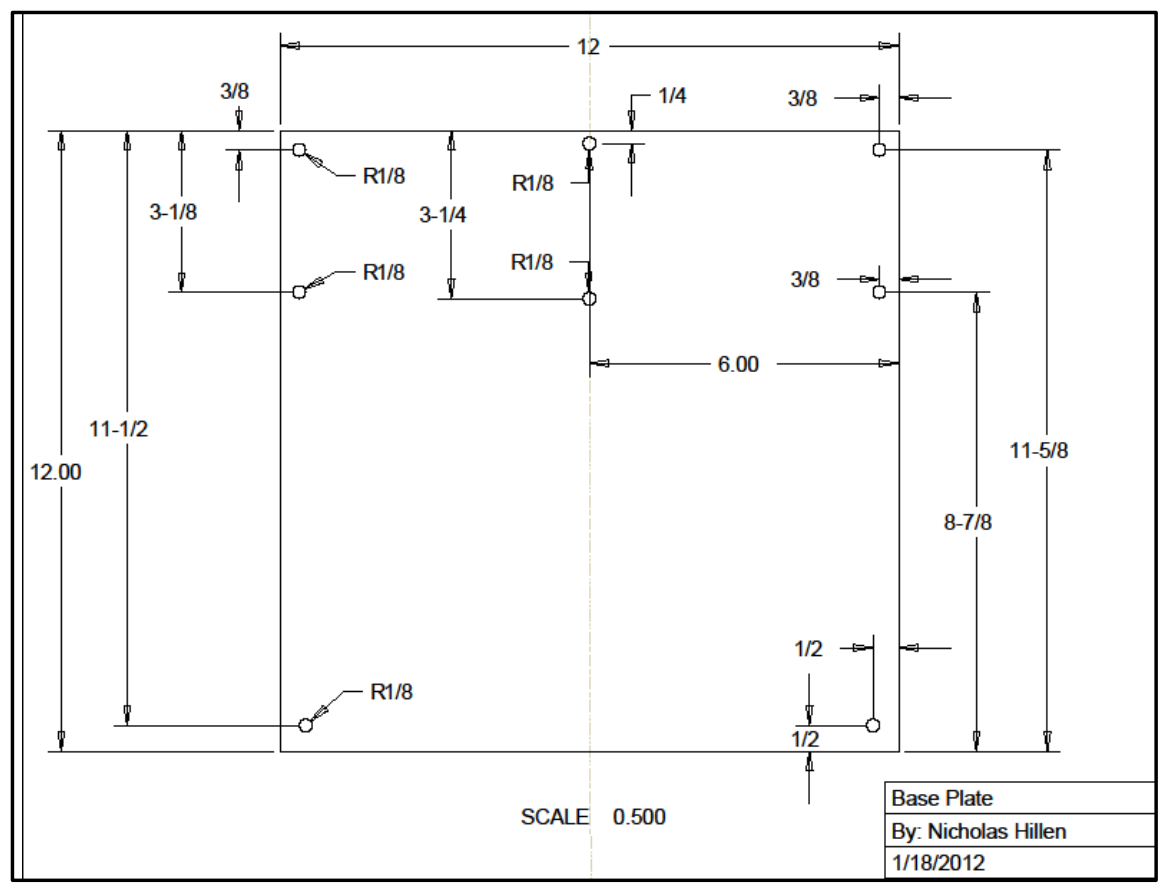

Figure A.1: Base mounting plate (0.25 in thick stainless steel sheet) used to attach to the leveling plate to the structural frame (units in inches).

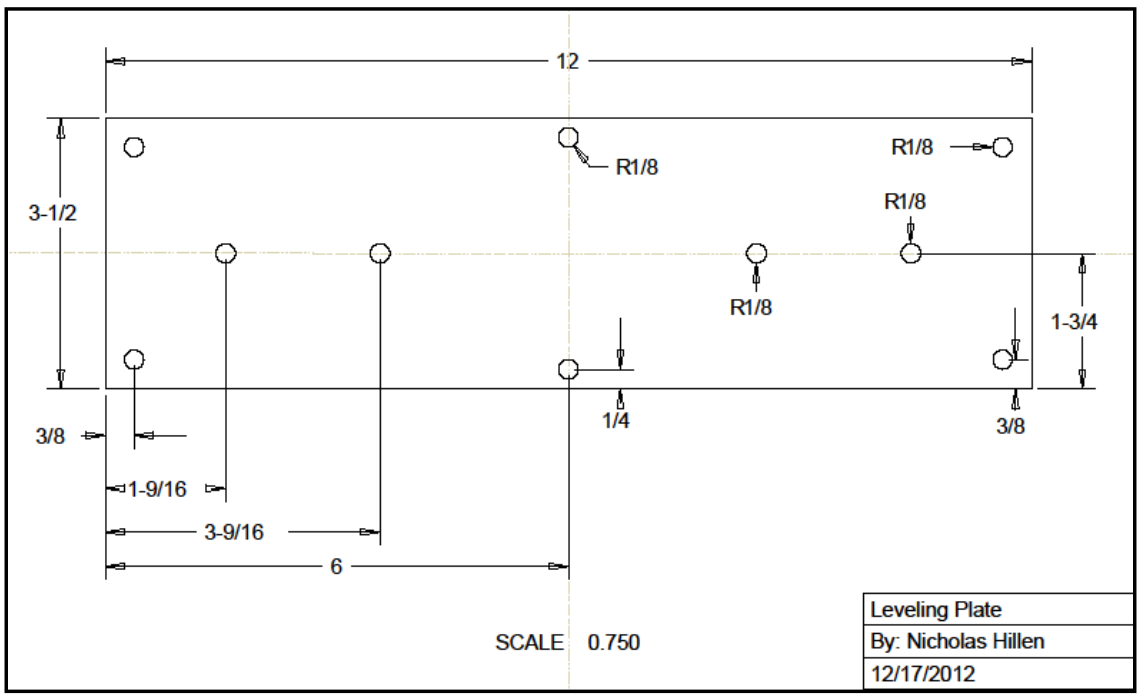

Figure A.2: Leveling plate (0.25 in thick stainless steel sheet) used to level the single droplet tank (units in inches). 


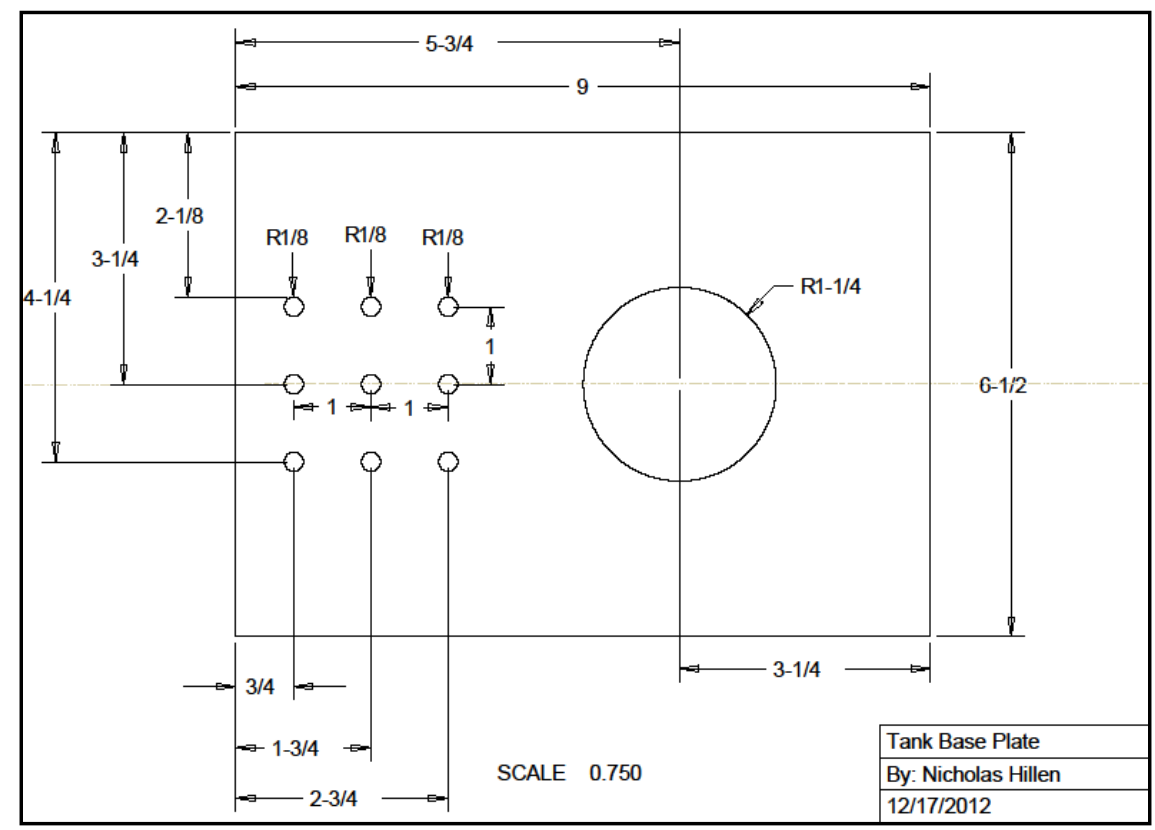

Figure A.3: Base plate (0.25 in thick stainless steel sheet) used to attach the acrylic tank to the micro-traverse (units in inches).

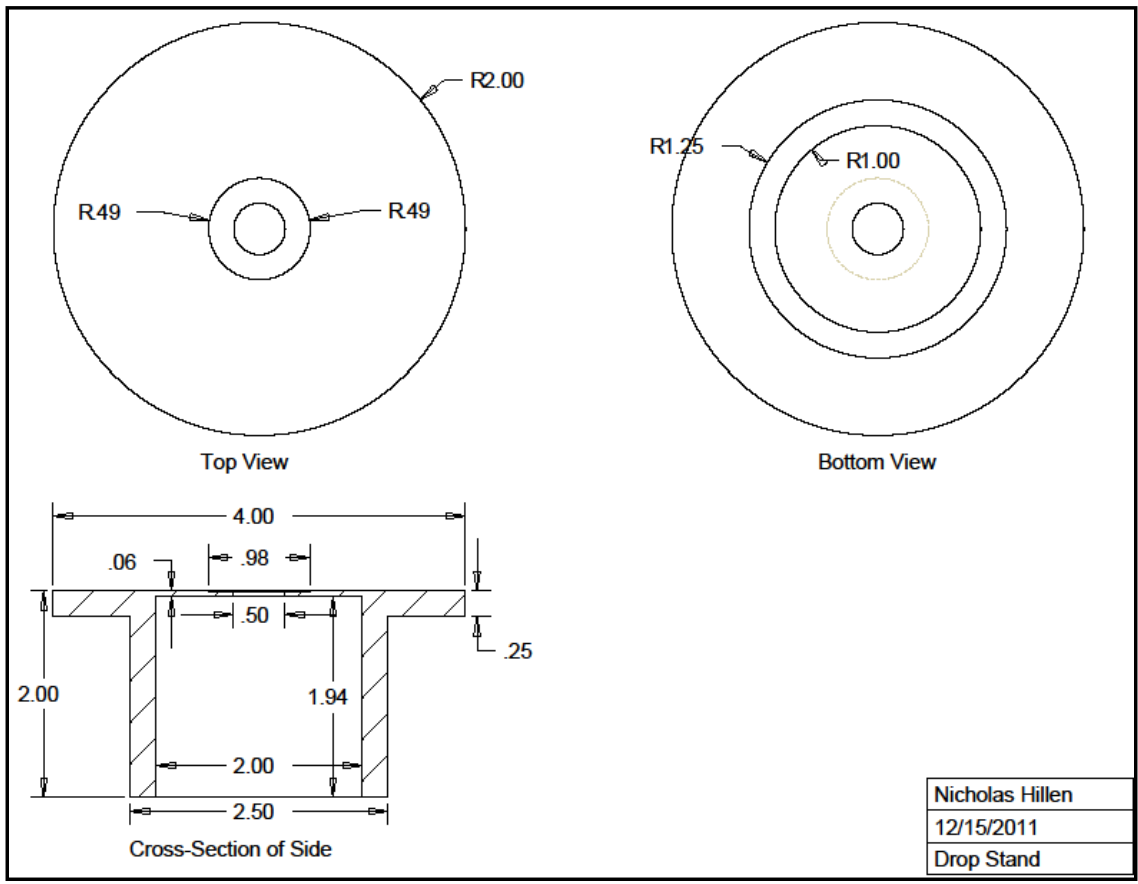

Figure A.4: Acrylic impact stand used for sub-cavity liquid film thickness experiments (units in inches). 


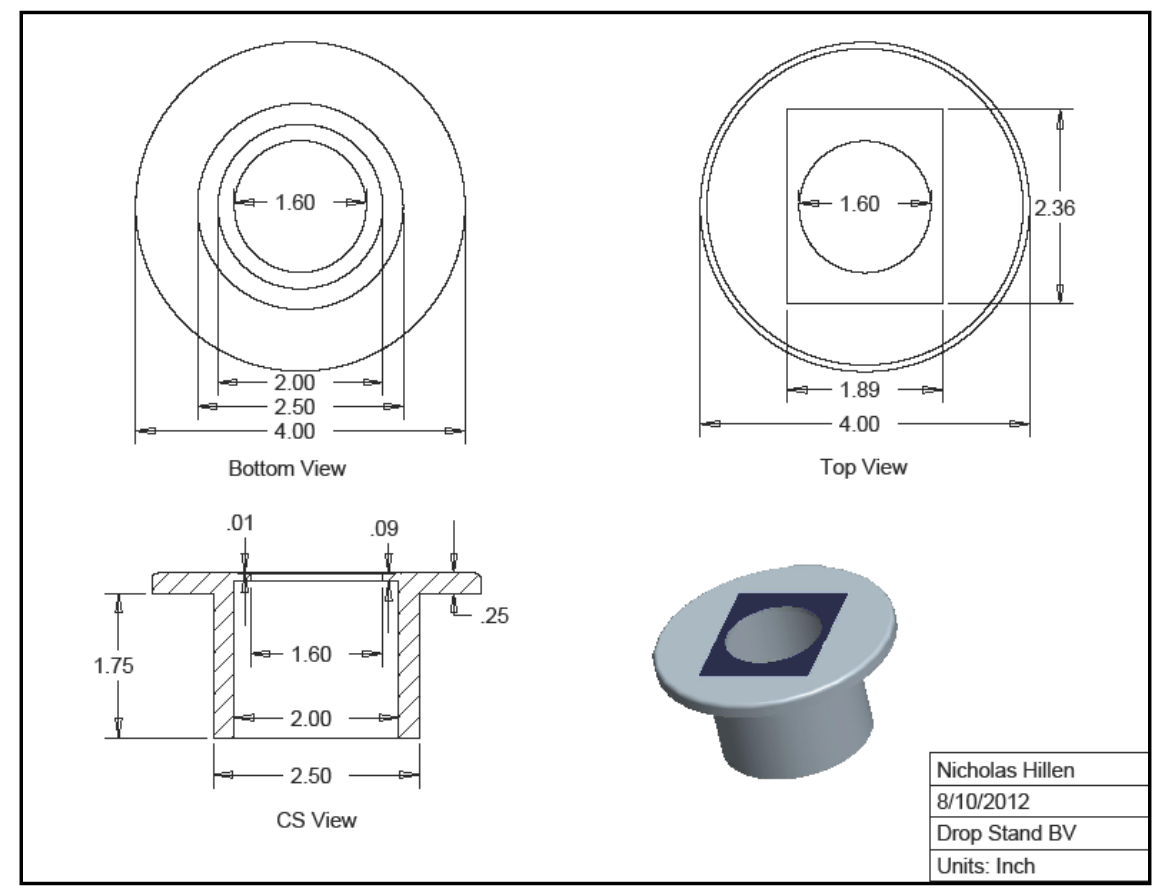

A.5: Acrylic impact stand with larger viewing port used for the BV sub-cavity radius experiments (units in inches).

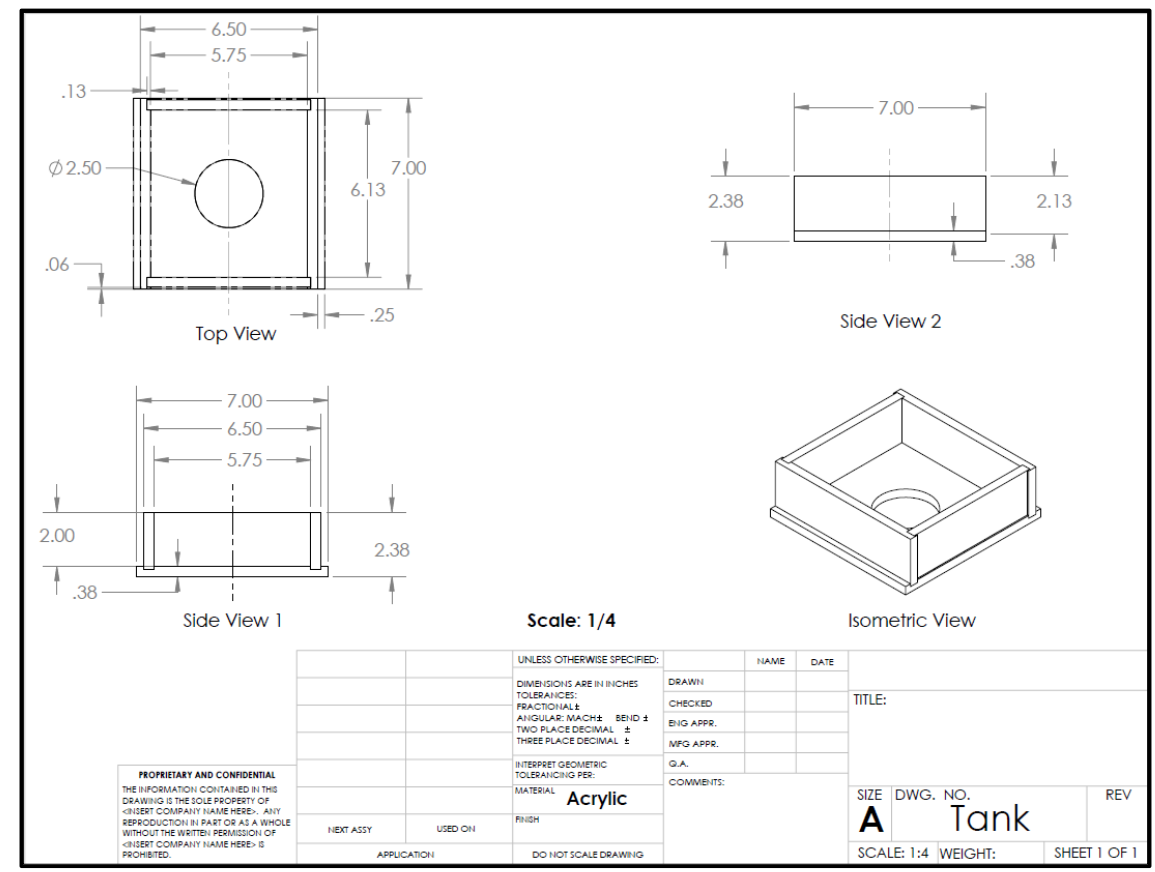

Figure A.6: Acrylic tank (units in inches). 


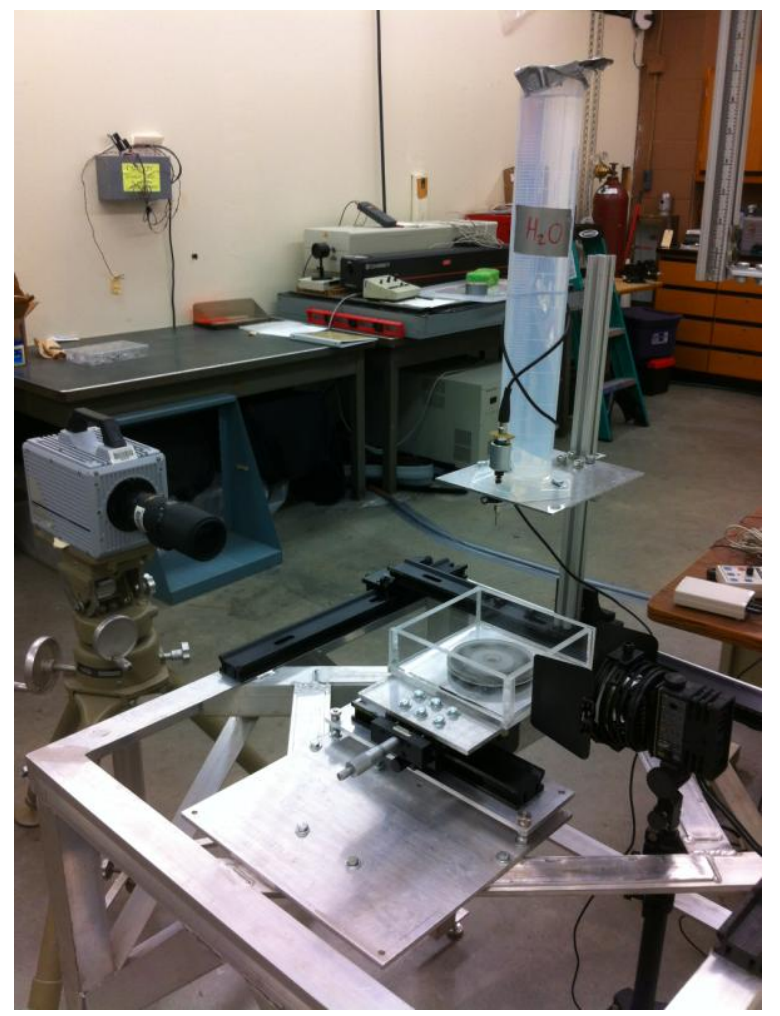

a)

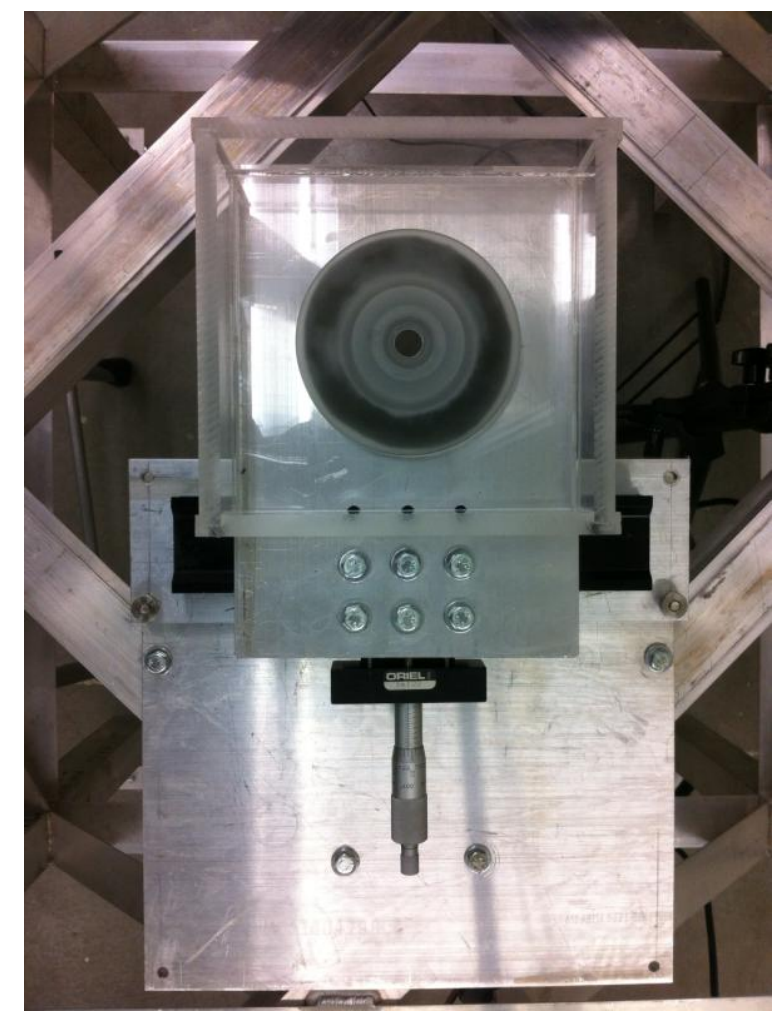

b)

Figure A.7: Final Single drop test facility Assembly. a) Backlit configuration. b) Tank and impact surface fixed to the micro-traverse. 


\section{Appendix B: $\quad$ Additional PDPA Spray Data (No Impact Surface)}

This Appendix contains the additional basic spray (no spray impact surface installed) PDPA data results for the various flow conditions not presented in Chapter 6. Included are the raw droplet diameter and velocity PDPA data histograms, and the average droplet diameter and velocity distributions. It should be noted that $y$-traverse profiles have been only measured at $z=3.81 \mathrm{~cm}$. 

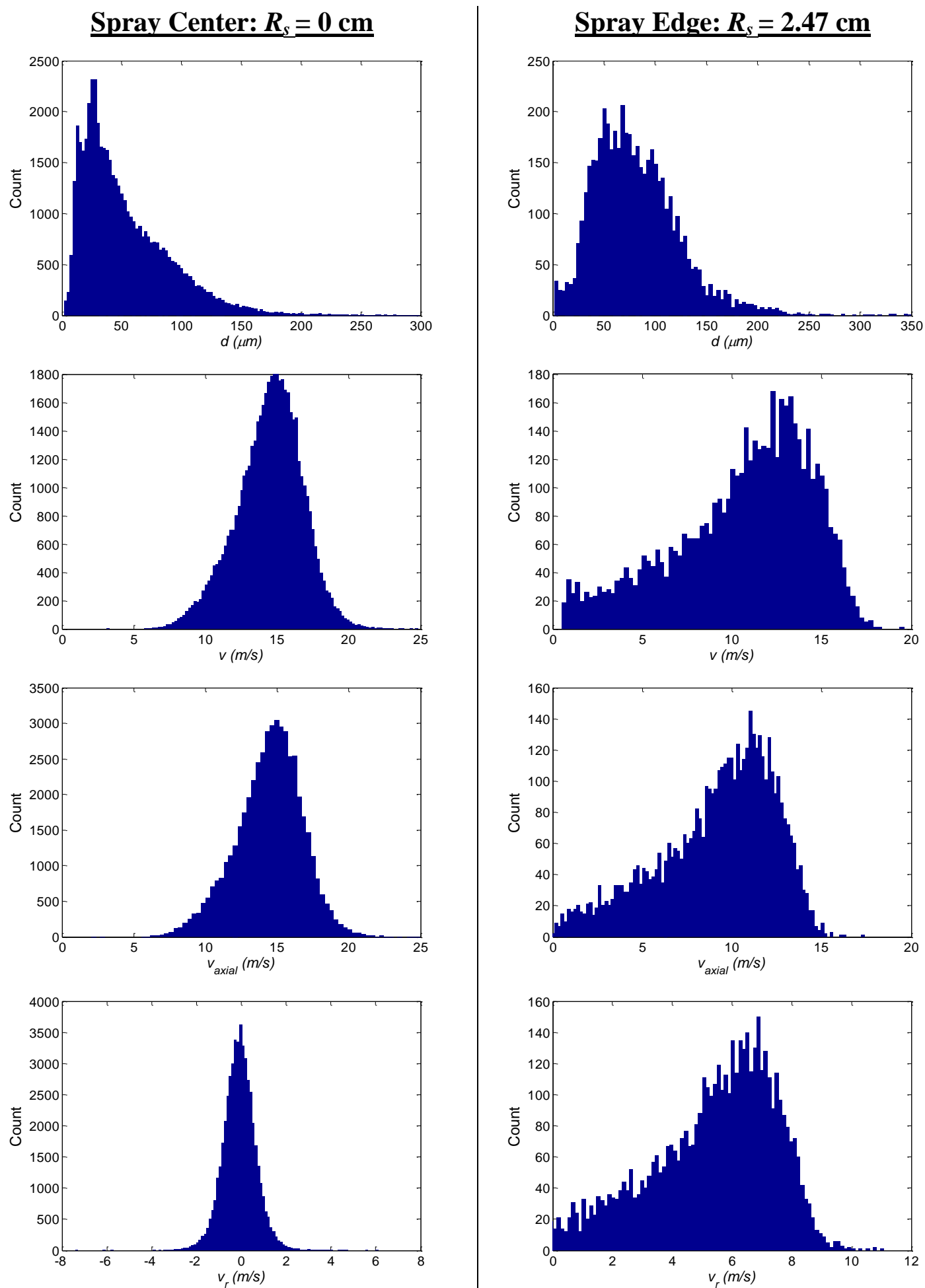

a)

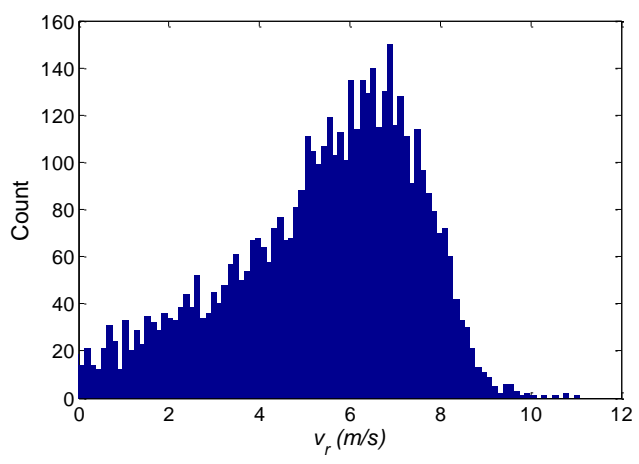

b)

Figure B.1: Spray droplet diameter, speed, axial velocity, and radial velocity for a 2.76 bar and $43.5 \mathrm{~L} / \mathrm{hr}$ at $z=3.81 \mathrm{~cm}$. a) Center of the spray, $R_{s}=0 \mathrm{~cm}$. b) Edge of spray, $R_{s}=2.47 \mathrm{~cm}$. 

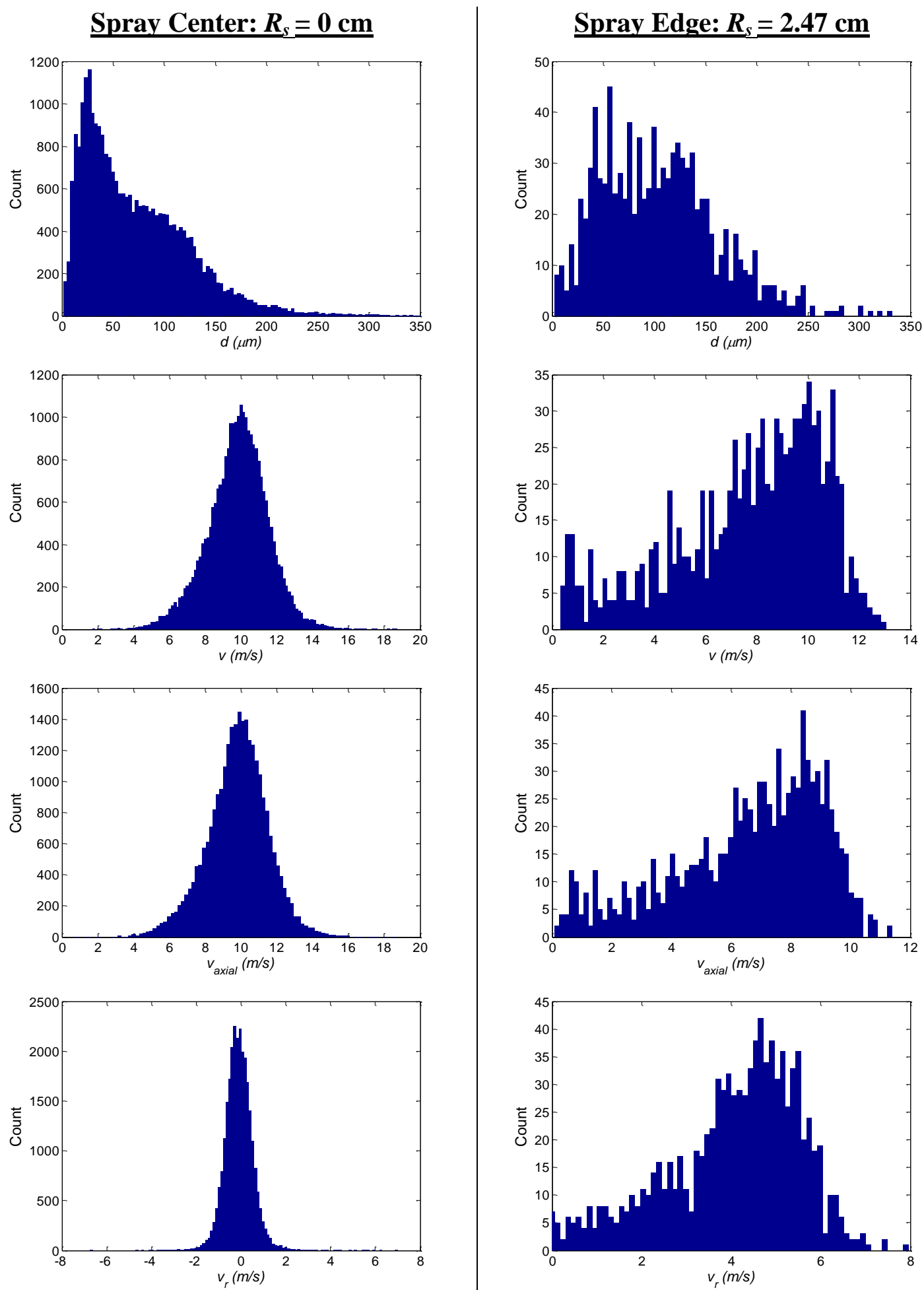

a)

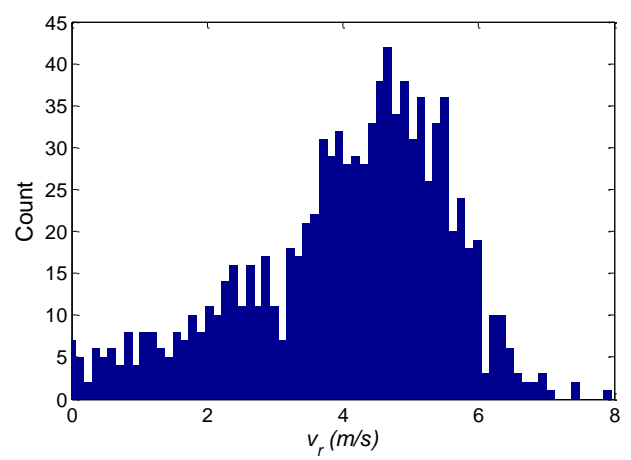

b)

Figure B.2: Spray droplet diameter, speed, axial velocity, and radial velocity for a 1.38 bar and $29.5 \mathrm{~L} / \mathrm{hr}$ at $z=3.81 \mathrm{~cm}$. a) Center of the spray, $R_{s}=0 \mathrm{~cm}$. b) Edge of spray, $R_{s}=2.47 \mathrm{~cm}$. 


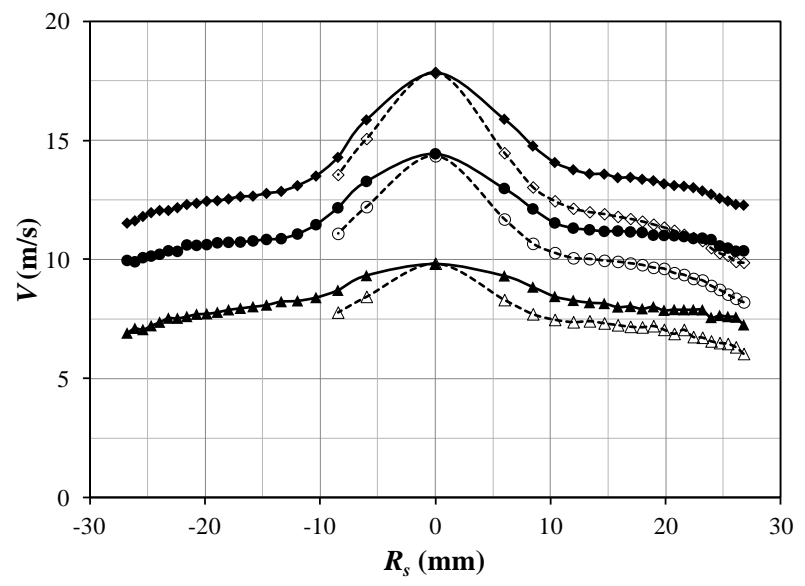

Figure B.3: Average droplet velocity magnitude profiles at a constant $z=3.81 \mathrm{~cm}$ for the different pressure cases $\Delta \triangle 1.38 \mathrm{bar}, 29.5 \mathrm{~L} / \mathrm{hr} ; \bullet \bigcirc 2.76 \mathrm{bar}, 43.5 \mathrm{~L} / \mathrm{hr} ; \diamond \diamond 4.14 \mathrm{bar}, 54.5$ $\mathrm{L} / \mathrm{hr}$; - $x$-plane data; ---- $y$-plane.

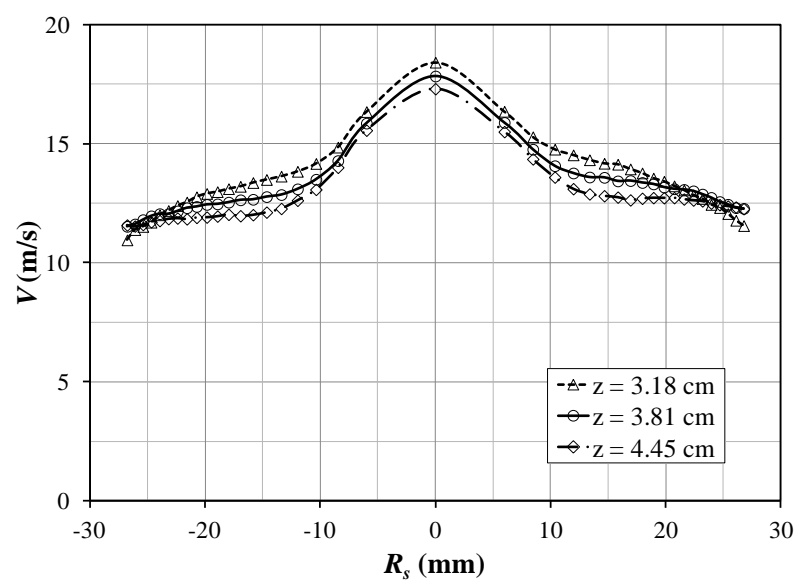

Figure B.4: Variation of the standoff distance of the measuring profile to the nozzle for a spray flow condition of the average velocity magnitude for $4.14 \mathrm{bar}$ and $54.5 \mathrm{~L} / \mathrm{hr}$. 

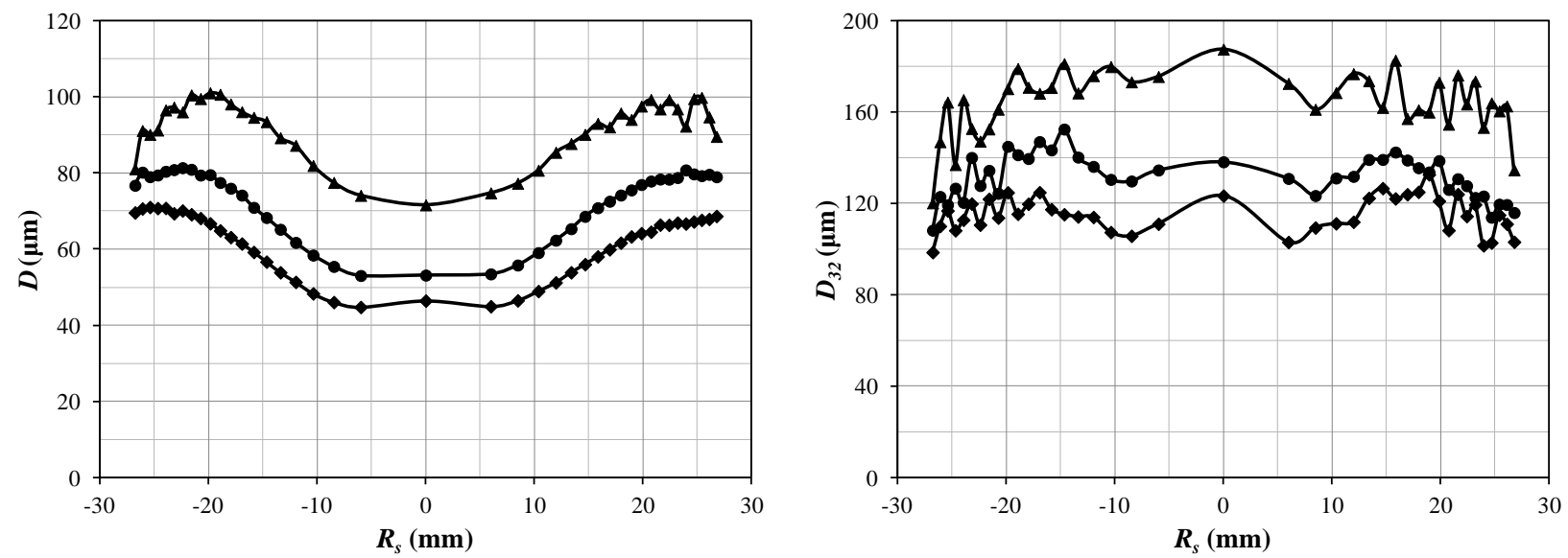

a)

b)
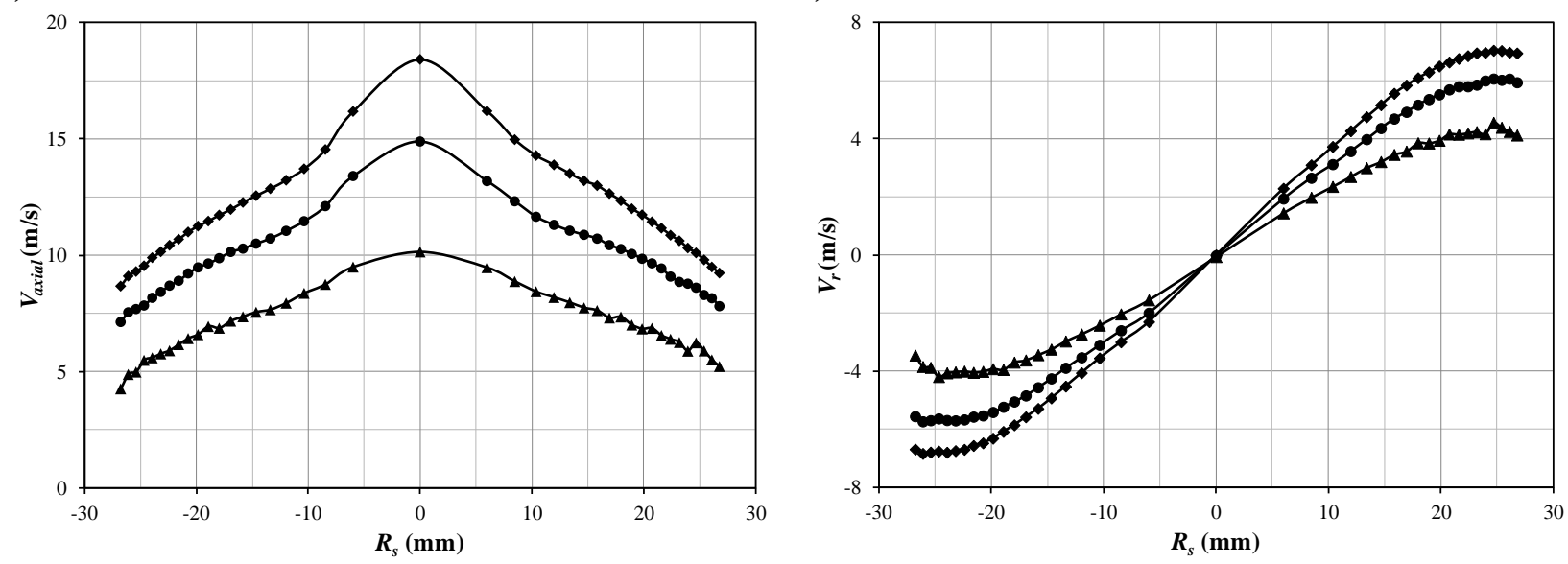

d)

c)

Figure B.5: Average droplet characteristic profiles at a constant $z=3.18 \mathrm{~cm}$ for the different pressure cases. a) Arithmetic mean diameter. b) Sauter mean diameter. c) Average axial velocity. d) Average radial velocity. e) Average velocity magnitude. $\Delta 1.38$ bar, $29.5 \mathrm{~L} / \mathrm{hr}$; 2.76 bar, $43.5 \mathrm{~L} / \mathrm{hr} ; 4$ 4.14 bar, $54.5 \mathrm{~L} / \mathrm{hr}$. 


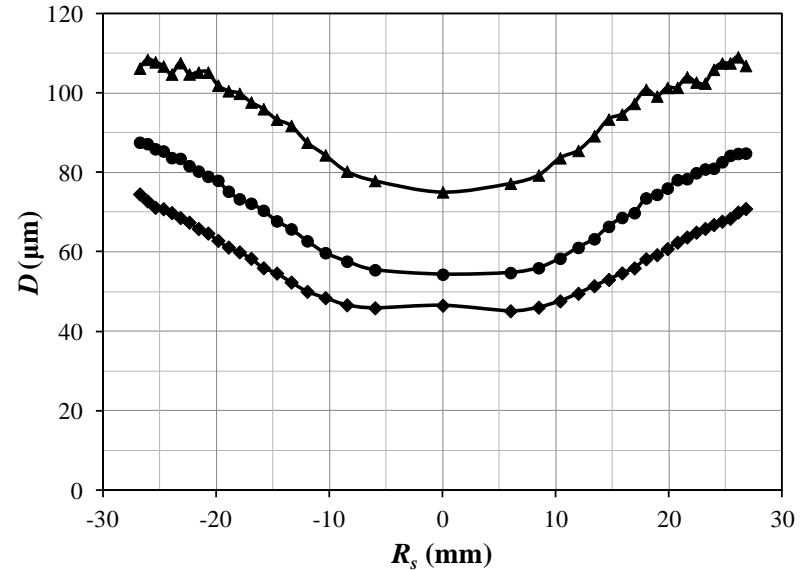

a)

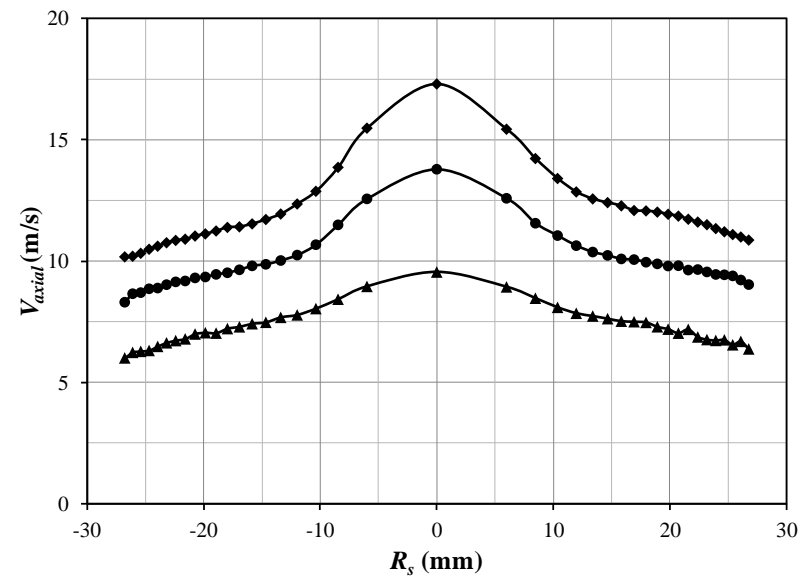

c)

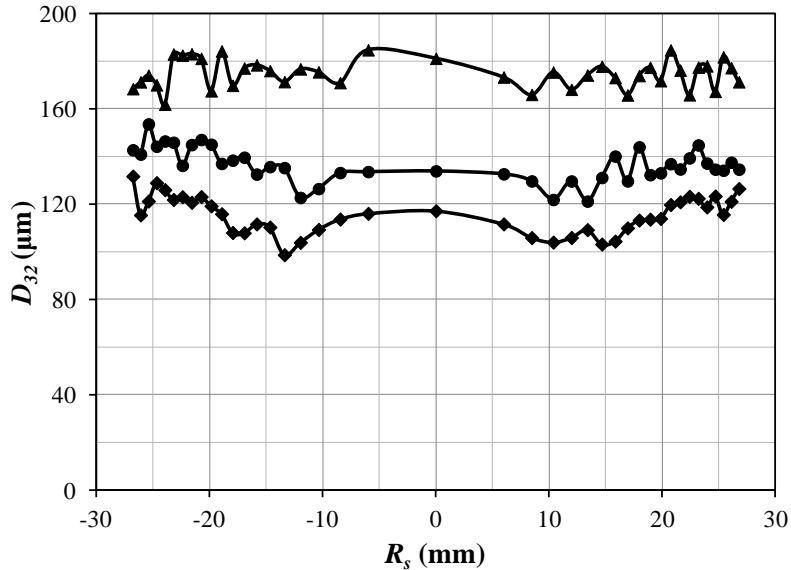

b)

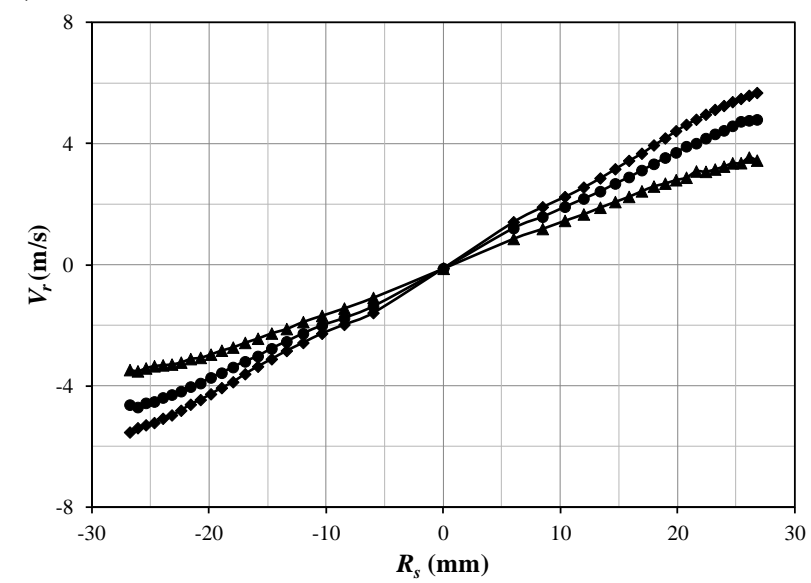

d)

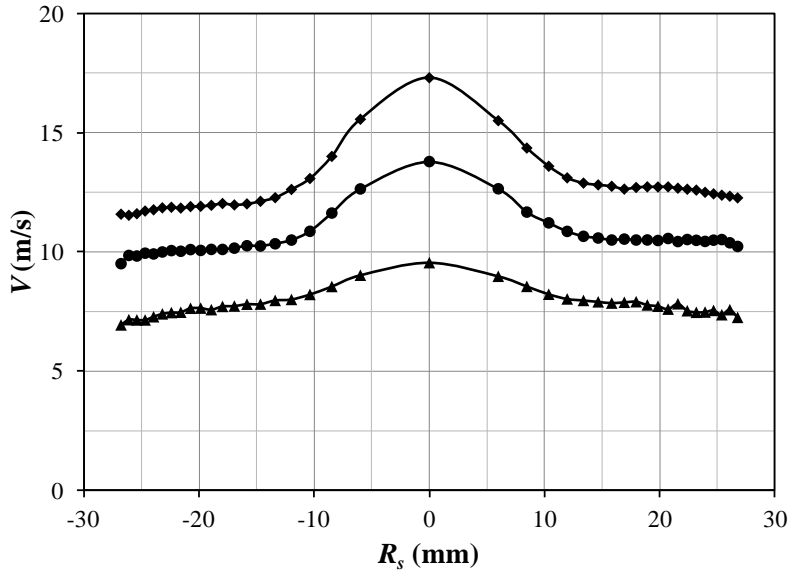

e)

Figure B.6: Average droplet characteristic profiles at a constant $z=4.45 \mathrm{~cm}$ for the different pressure cases. a) Arithmetic mean diameter. b) Sauter mean diameter. c) Average axial velocity. d) Average radial velocity. e) Average velocity magnitude. $\triangle 1.38 \mathrm{bar}, 29.5 \mathrm{~L} / \mathrm{hr}$; 2.76 bar, 43.5 L/hr; 4.14 bar, 54.5 L/hr. 


\section{Appendix C: Additional PDPA Splashing Data(With Impact Surface)}

Contained within this appendix are additional plots of the spray PDPA data collected with the spray impact surface installed to generate splashing. Additional PDPA data of the droplet diameter and velocity histograms separated into the spray, ejected splash, return splash, and absolute splash components for flow conditions listed Table 3.3 are contained herein. Also included are the PDPA splashed droplet characteristic profiles for both the $x$-axis and vertical edge traverses 

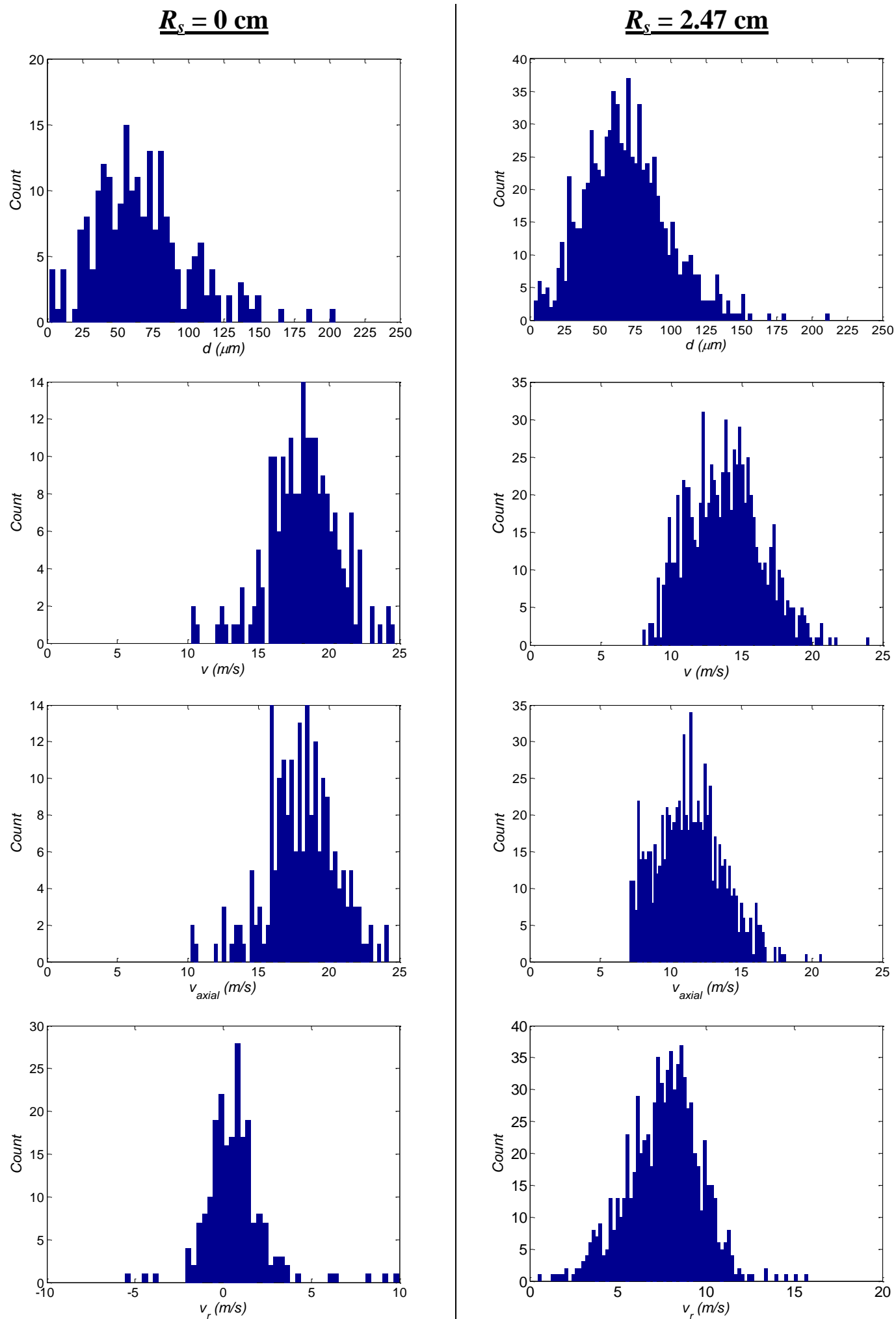

a)

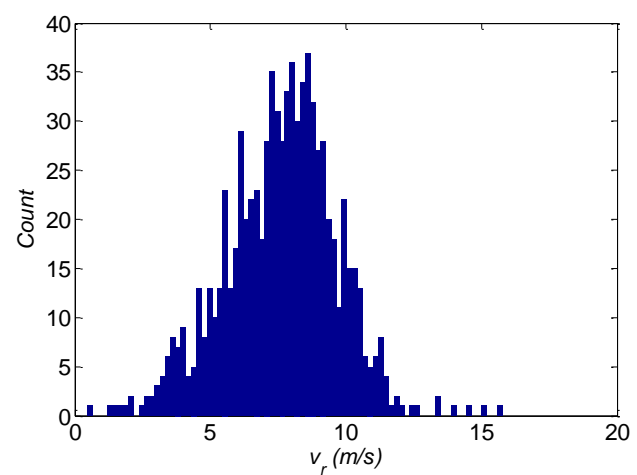

b)

Figure C.1: Histograms of the incoming spray droplet data for $4.14 \mathrm{bar}, 54.5 \mathrm{~L} / \mathrm{hr}$ at $z=3.65$ cm at a single point. a) $R_{s}=0 \mathrm{~cm}$. b) $R_{s}=2.47 \mathrm{~cm}$. 

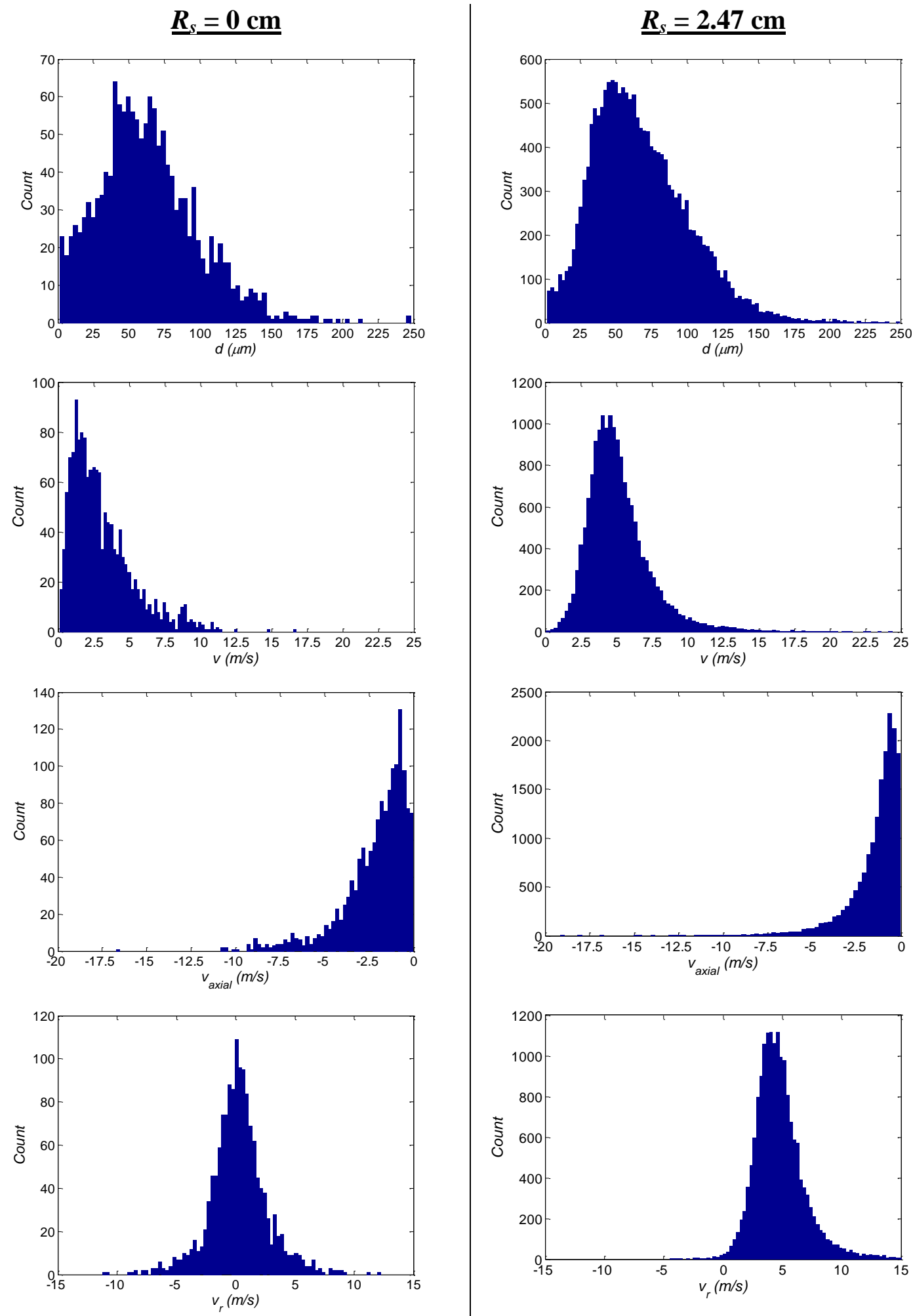

a)

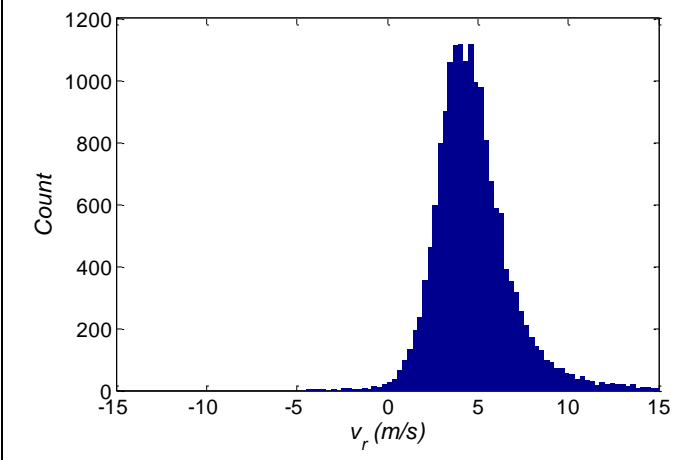

b)

Figure C.2: Histograms of the ejected splashed droplet data for $4.14 \mathrm{bar}, 54.5 \mathrm{~L} / \mathrm{hr}$ at $z=3.65$ cm at a single point. a) $R_{s}=0 \mathrm{~cm}$. b) $R_{s}=2.47 \mathrm{~cm}$. 

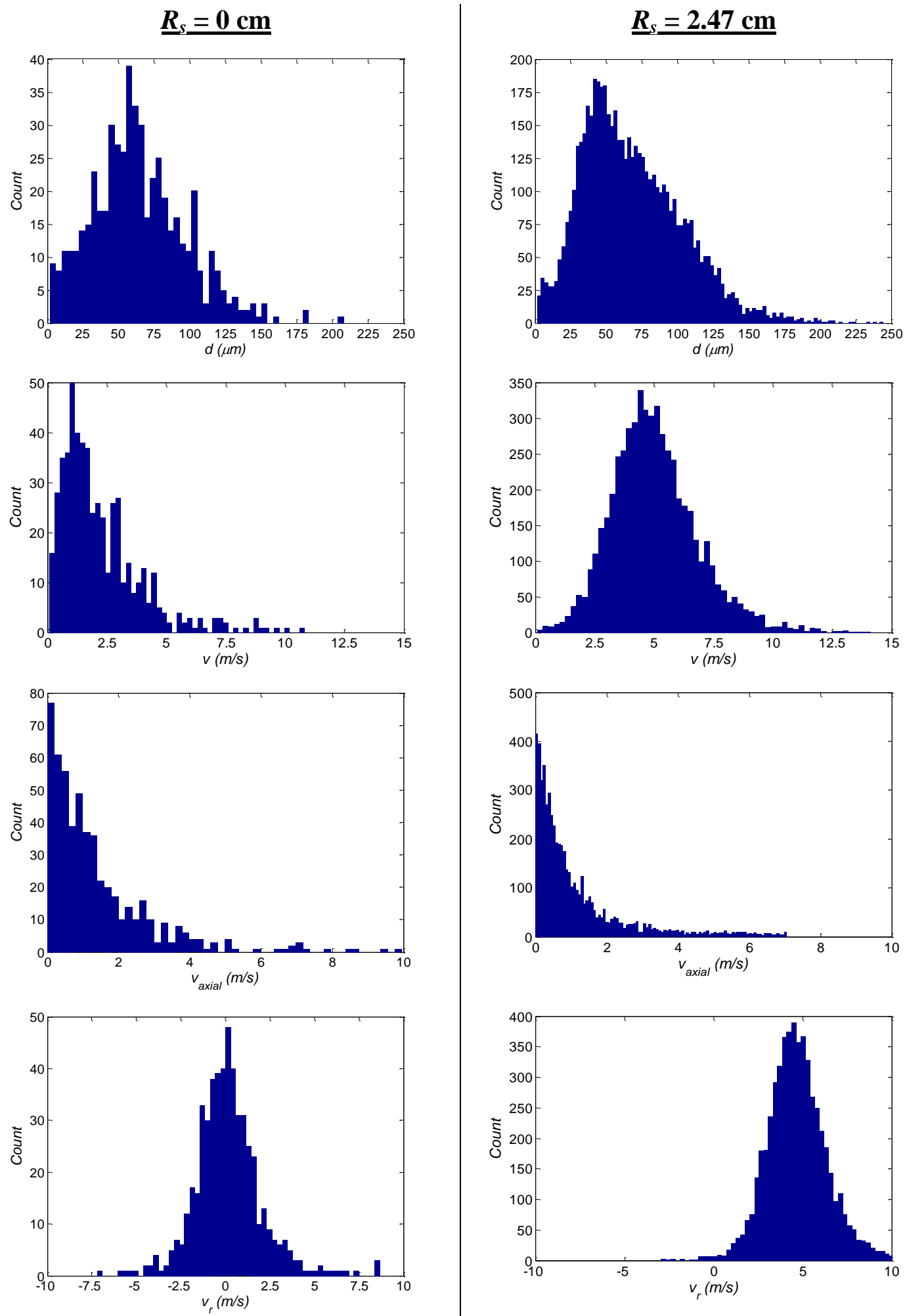

a)

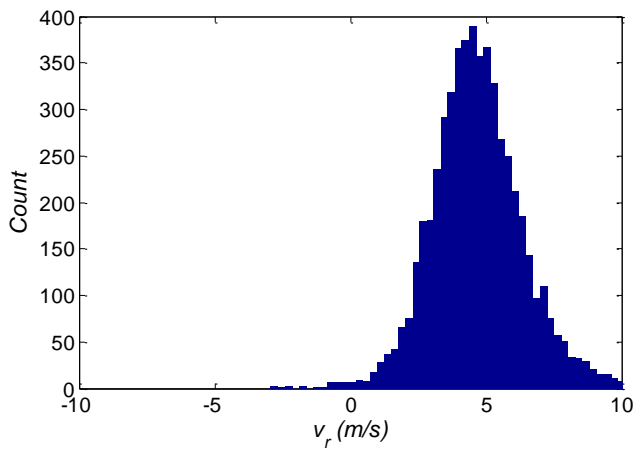

b)

Figure C.3: Histograms of the returning splashed droplet data for $4.14 \mathrm{bar}, 54.5 \mathrm{~L} / \mathrm{hr}$ at $z=$ $3.65 \mathrm{~cm}$ at a single point. a) $R_{s}=0 \mathrm{~cm}$. b) $R_{s}=2.47 \mathrm{~cm}$. 

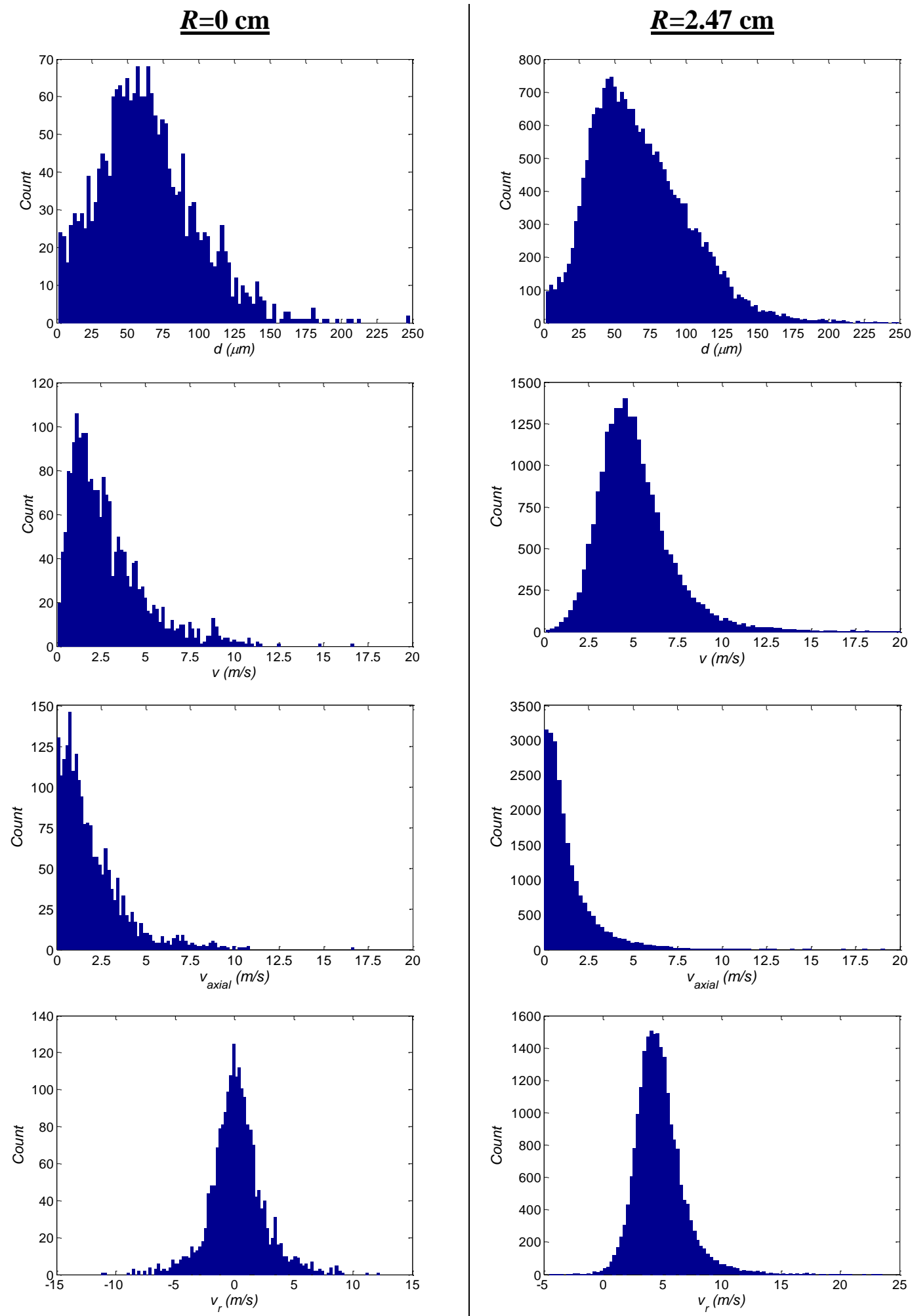

a)

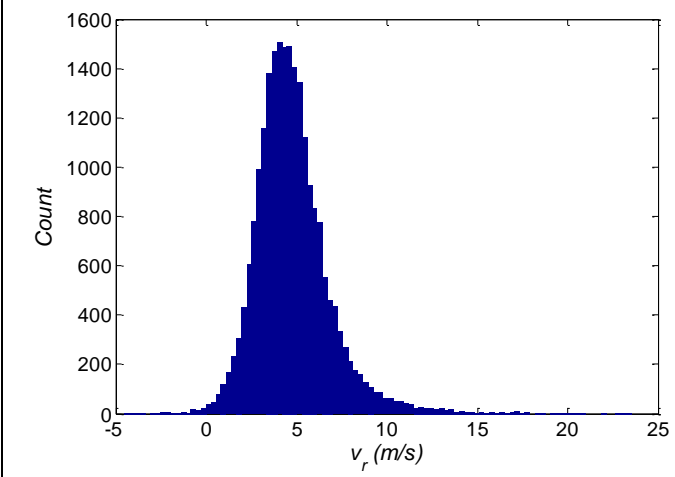

b)

Figure C.4: Histograms of the absolute splashed droplet data for $4.14 \mathrm{bar}, 54.5 \mathrm{~L} / \mathrm{hr}$ at $z=3.65$ cm at a single point. a) $R_{s}=0 \mathrm{~cm}$. b) $R_{s}=2.47 \mathrm{~cm}$. 

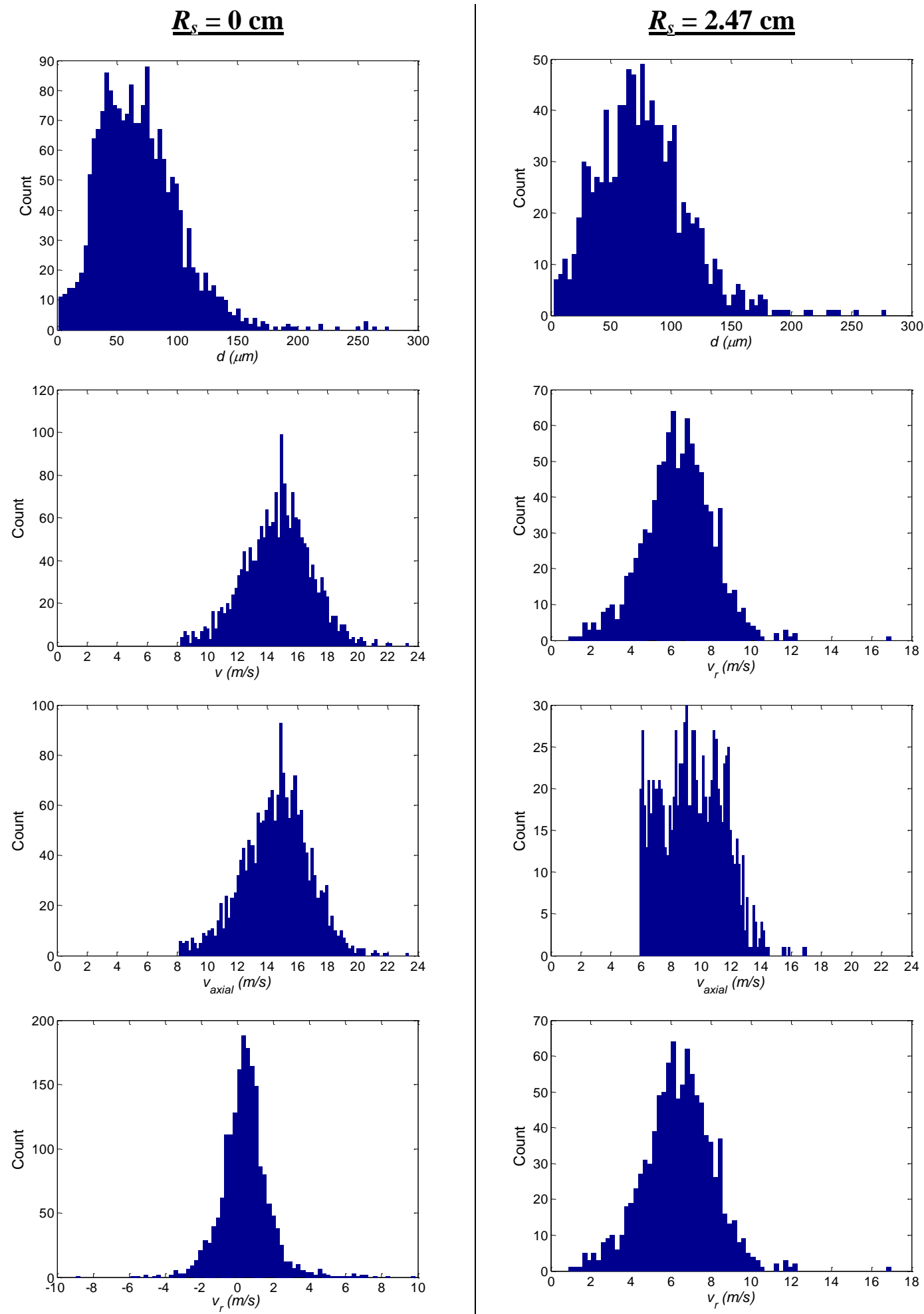

a)

b)

Figure C.5: Histograms of the incoming spray droplet data for $2.76 \mathrm{bar}, 43.5 \mathrm{~L} / \mathrm{hr}$ at $z=3.65$ cm at a single point. a) $R_{s}=0 \mathrm{~cm}$. b) $R_{s}=2.47 \mathrm{~cm}$. 

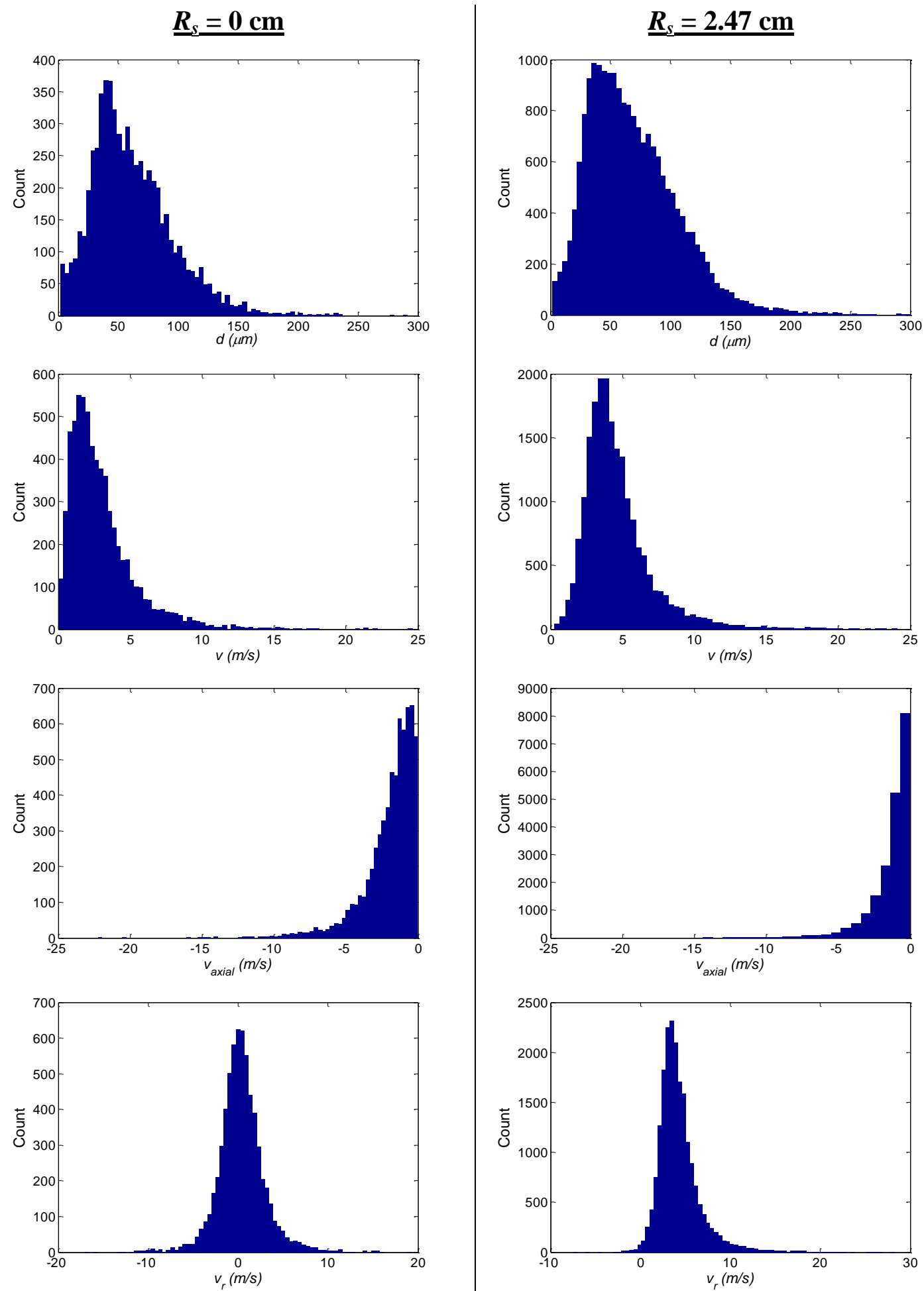

a)

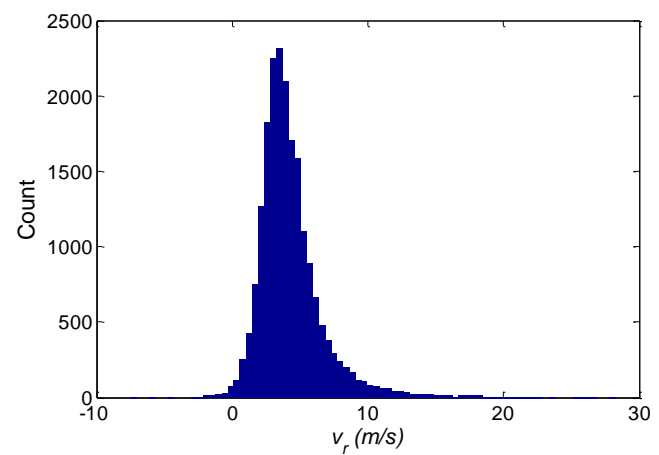

b)

Figure C.6: Histograms of the ejected splashed droplet data for $2.76 \mathrm{bar}, 43.5 \mathrm{~L} / \mathrm{hr}$ at $z=3.65$ cm at a single point. a) $R_{s}=0 \mathrm{~cm}$. b) $R_{s}=2.47 \mathrm{~cm}$. 

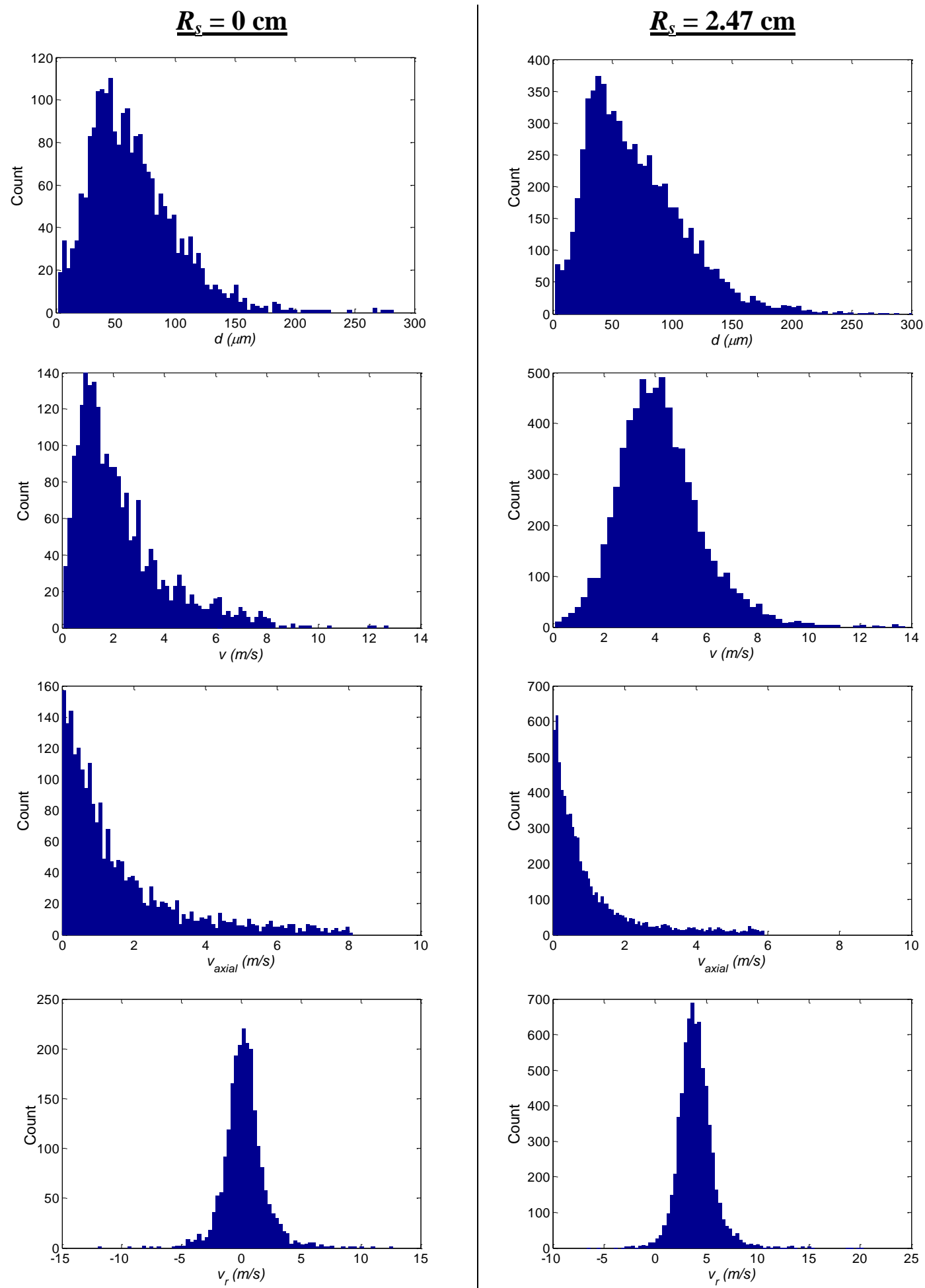

a)

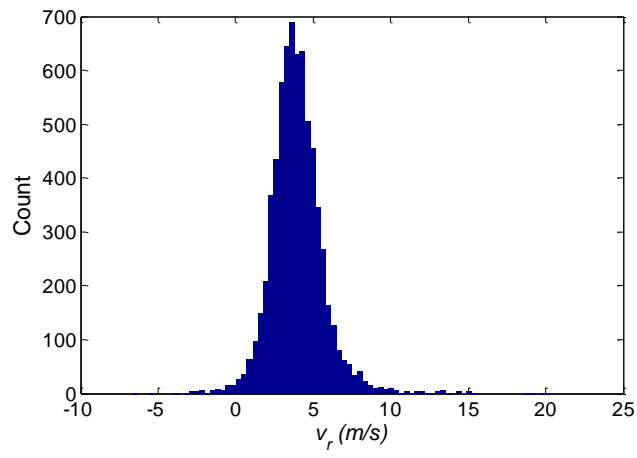

b)

Figure C.7: Histograms of the returning splashed droplet data for $2.76 \mathrm{bar}, 43.5 \mathrm{~L} / \mathrm{hr}$ at $z=$ $3.65 \mathrm{~cm}$ at a single point. a) $R_{s}=0 \mathrm{~cm}$. b) $R_{s}=2.47 \mathrm{~cm}$. 

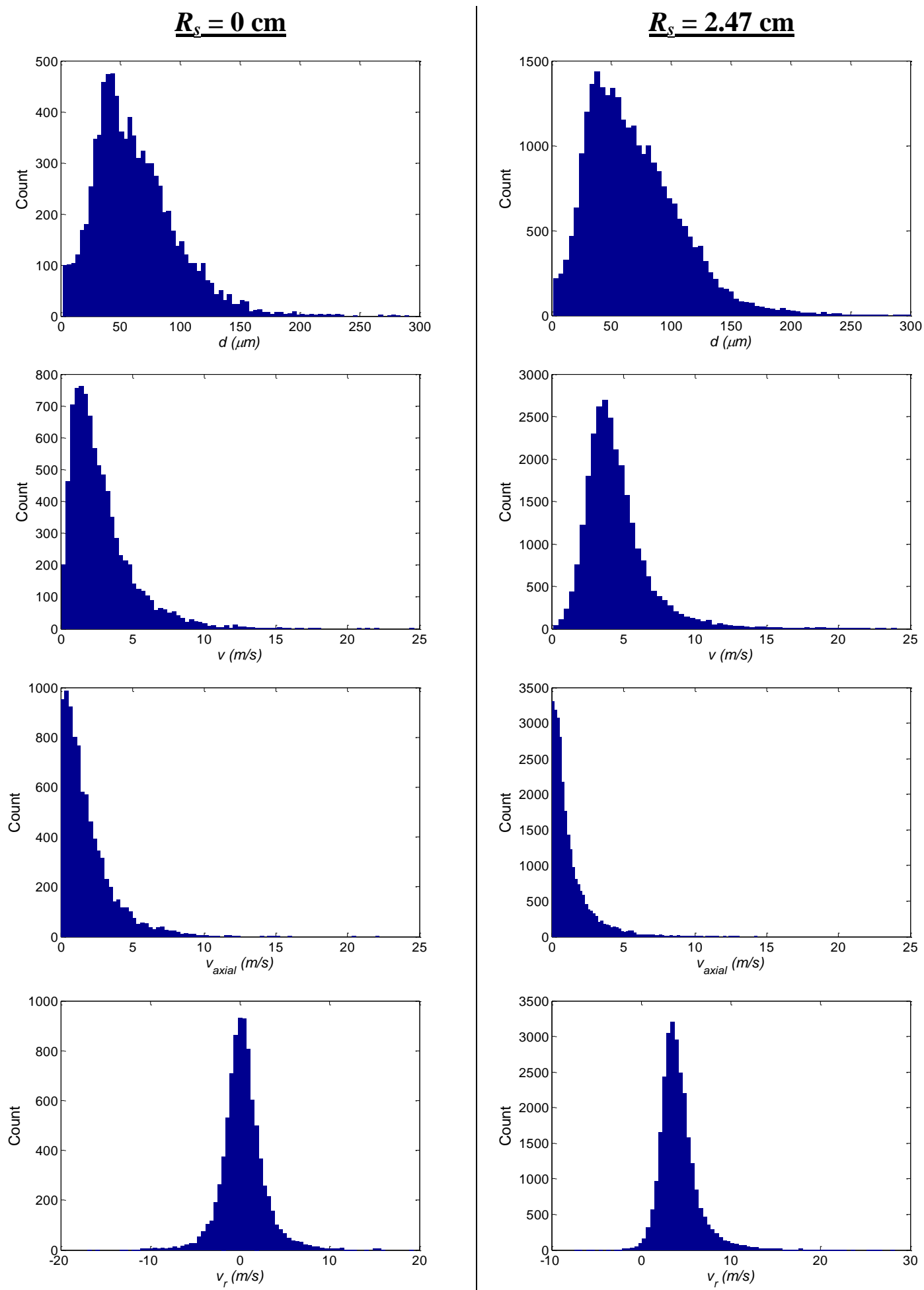

a)

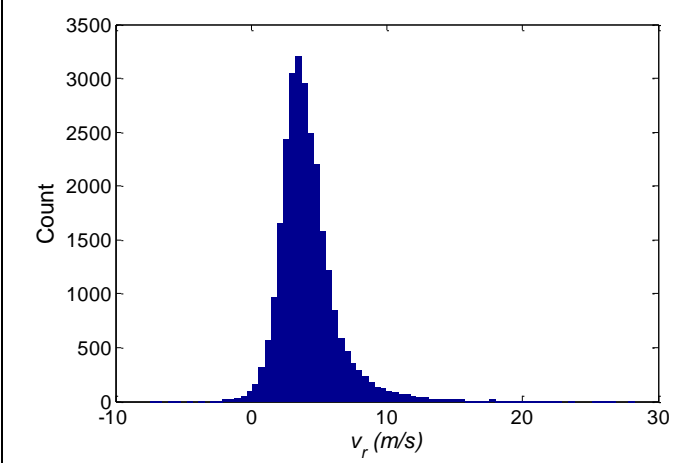

b)

Figure C.8: Histograms of the absolute splashed droplet data for $2.76 \mathrm{bar}, 43.5 \mathrm{~L} / \mathrm{hr}$ at $z=3.65$ cm at a single point. a) $R_{s}=0 \mathrm{~cm}$. b) $R_{s}=2.47 \mathrm{~cm}$. 

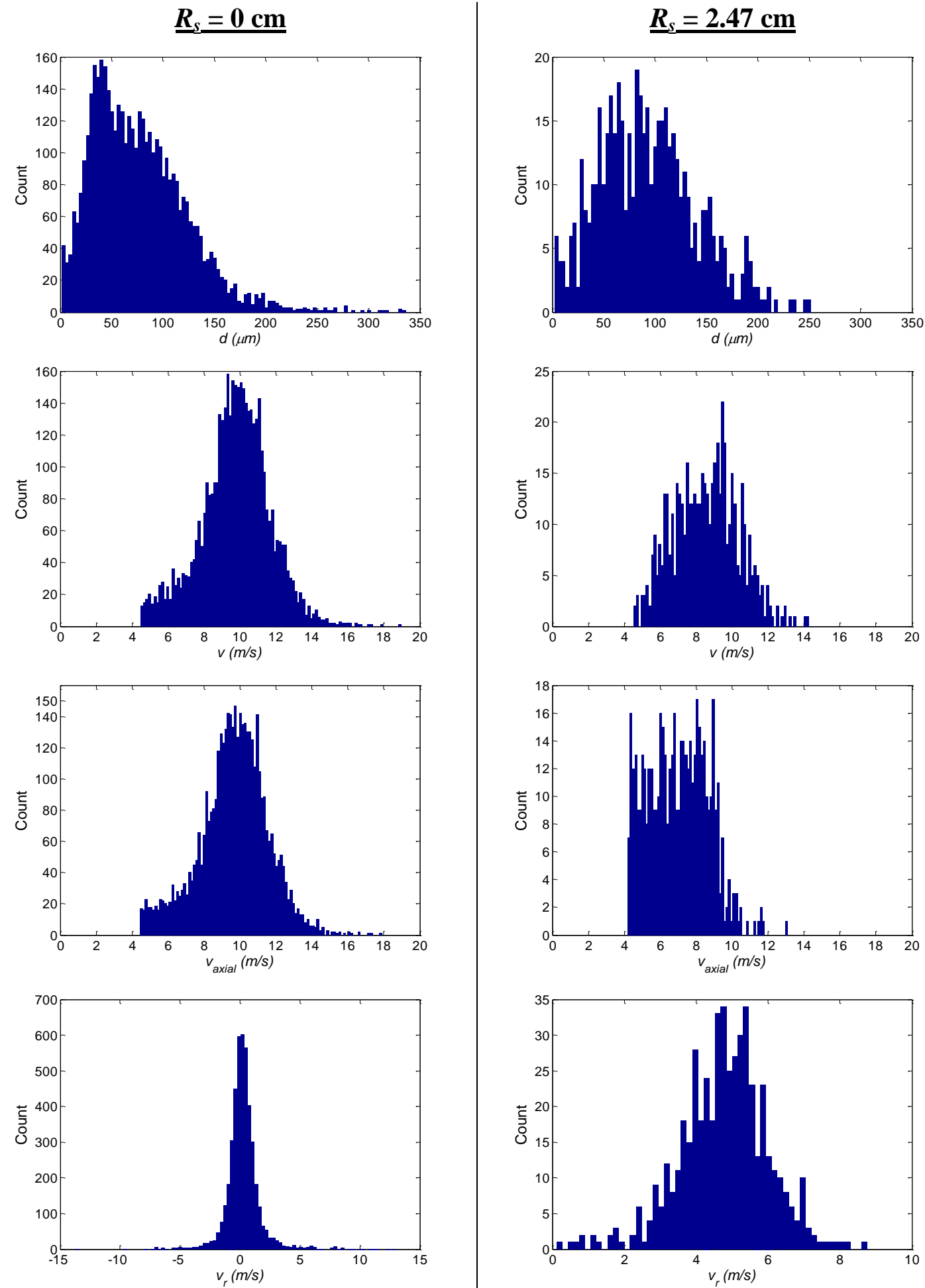

a)

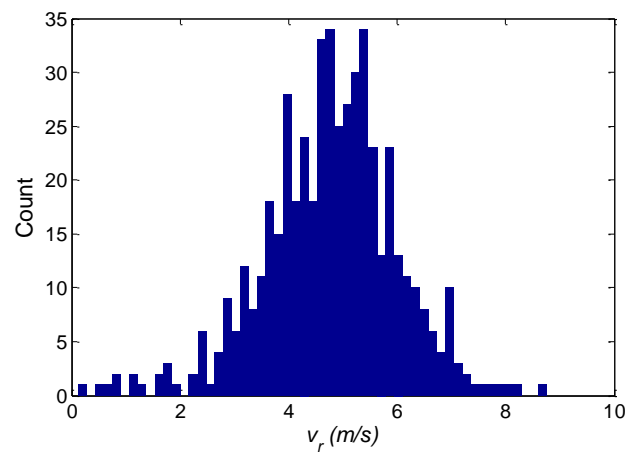

b)

Figure C.9: Histograms of the incoming spray droplet data for $1.38 \mathrm{bar}, 29.5 \mathrm{~L} / \mathrm{hr}$ at $z=3.65$ cm at a single point. a) $R_{s}=0 \mathrm{~cm}$. b) $R_{s}=2.47 \mathrm{~cm}$. 

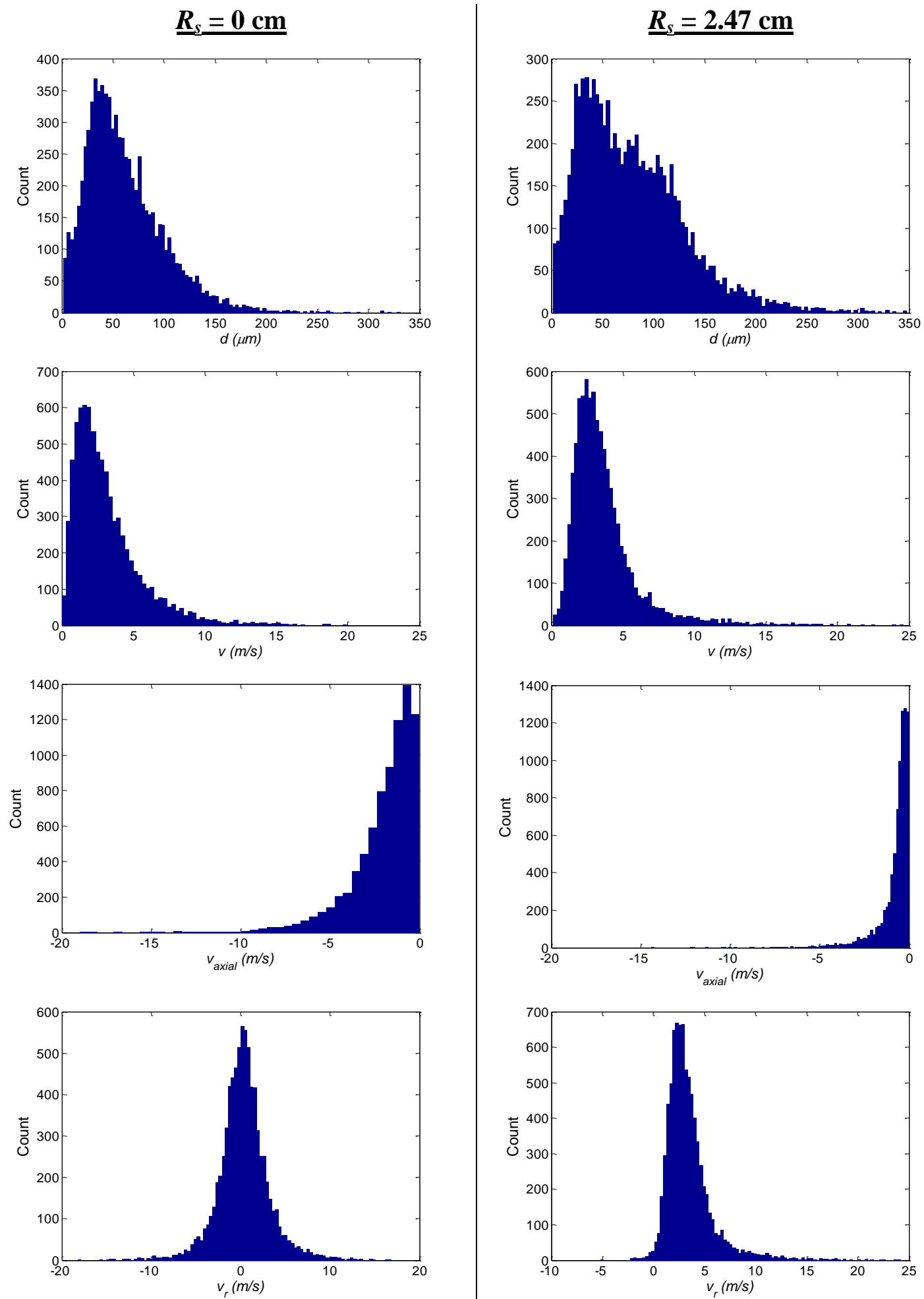

a)

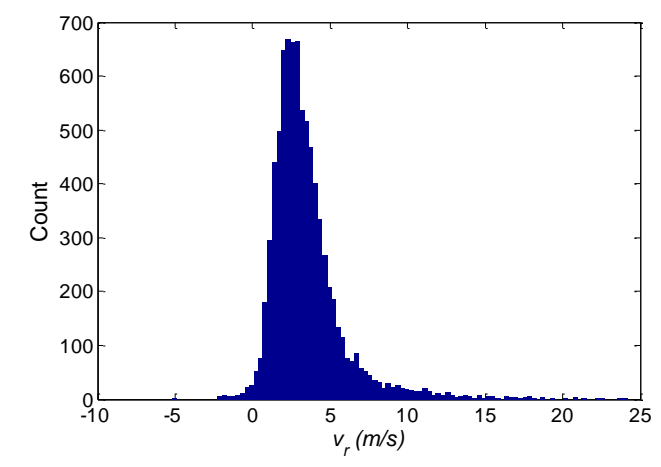

b)

Figure C.10: Histograms of the ejected splashed droplet data for $1.38 \mathrm{bar}, 29.5 \mathrm{~L} / \mathrm{hr}$ at $z=3.65$ cm at a single point. a) $R_{s}=0 \mathrm{~cm}$. b) $R_{s}=2.47 \mathrm{~cm}$. 

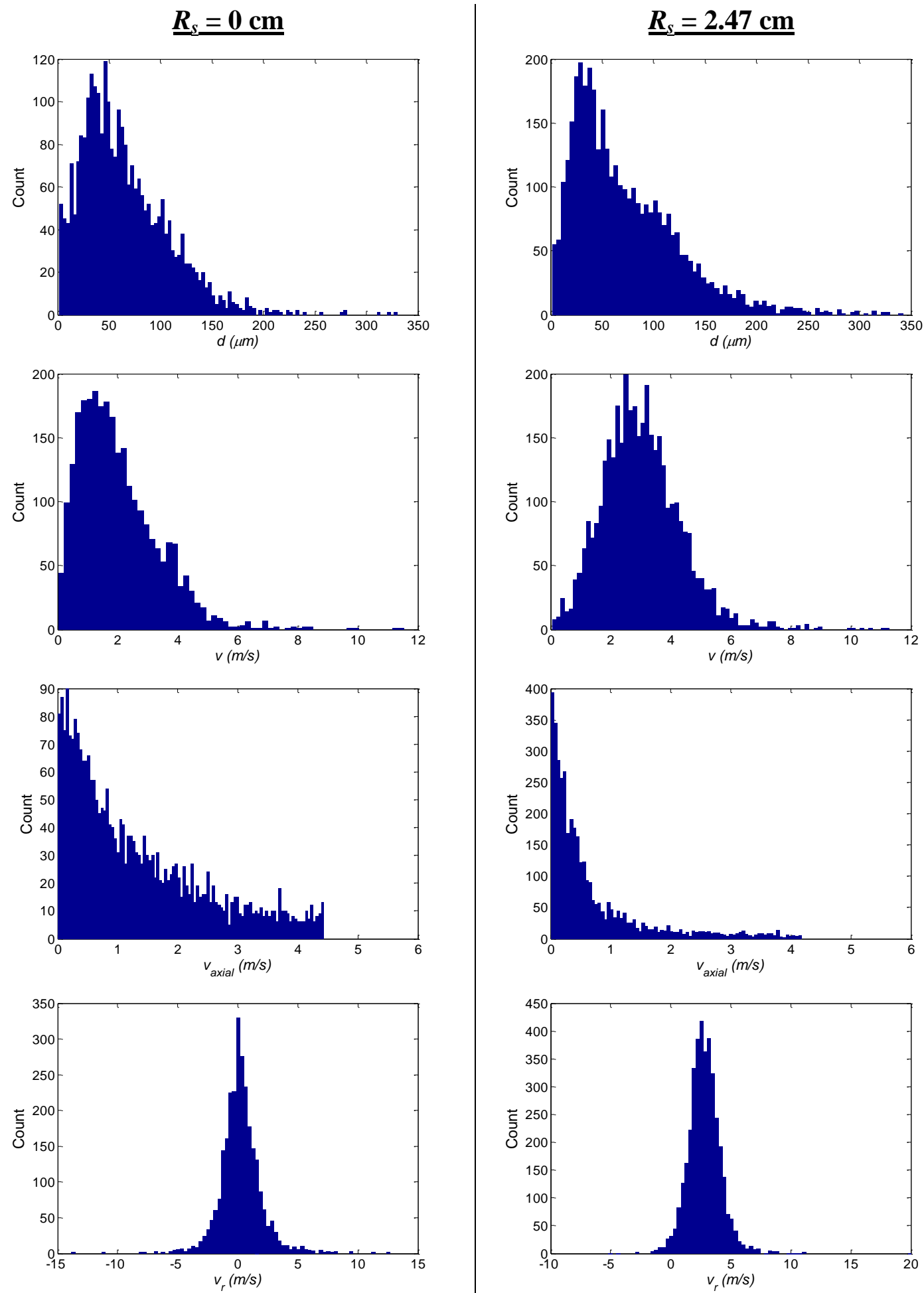

a)

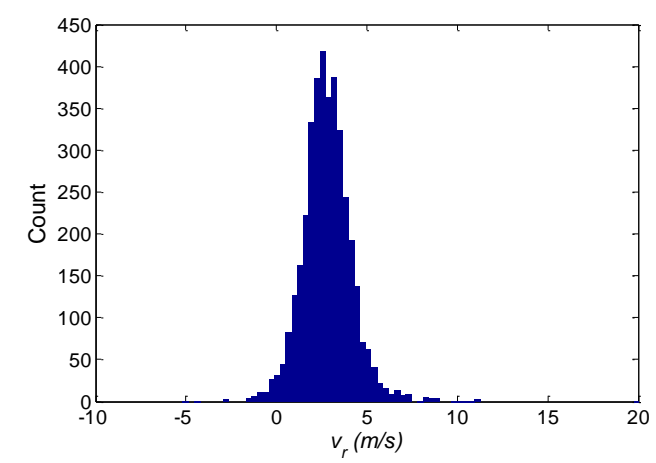

b)

Figure C.11: Histograms of the returning splashed droplet data for $1.38 \mathrm{bar}, 29.5 \mathrm{~L} / \mathrm{hr}$ at $z=$ $3.65 \mathrm{~cm}$ at a single point. a) $R_{s}=0 \mathrm{~cm}$. b) $R_{s}=2.47 \mathrm{~cm}$. 

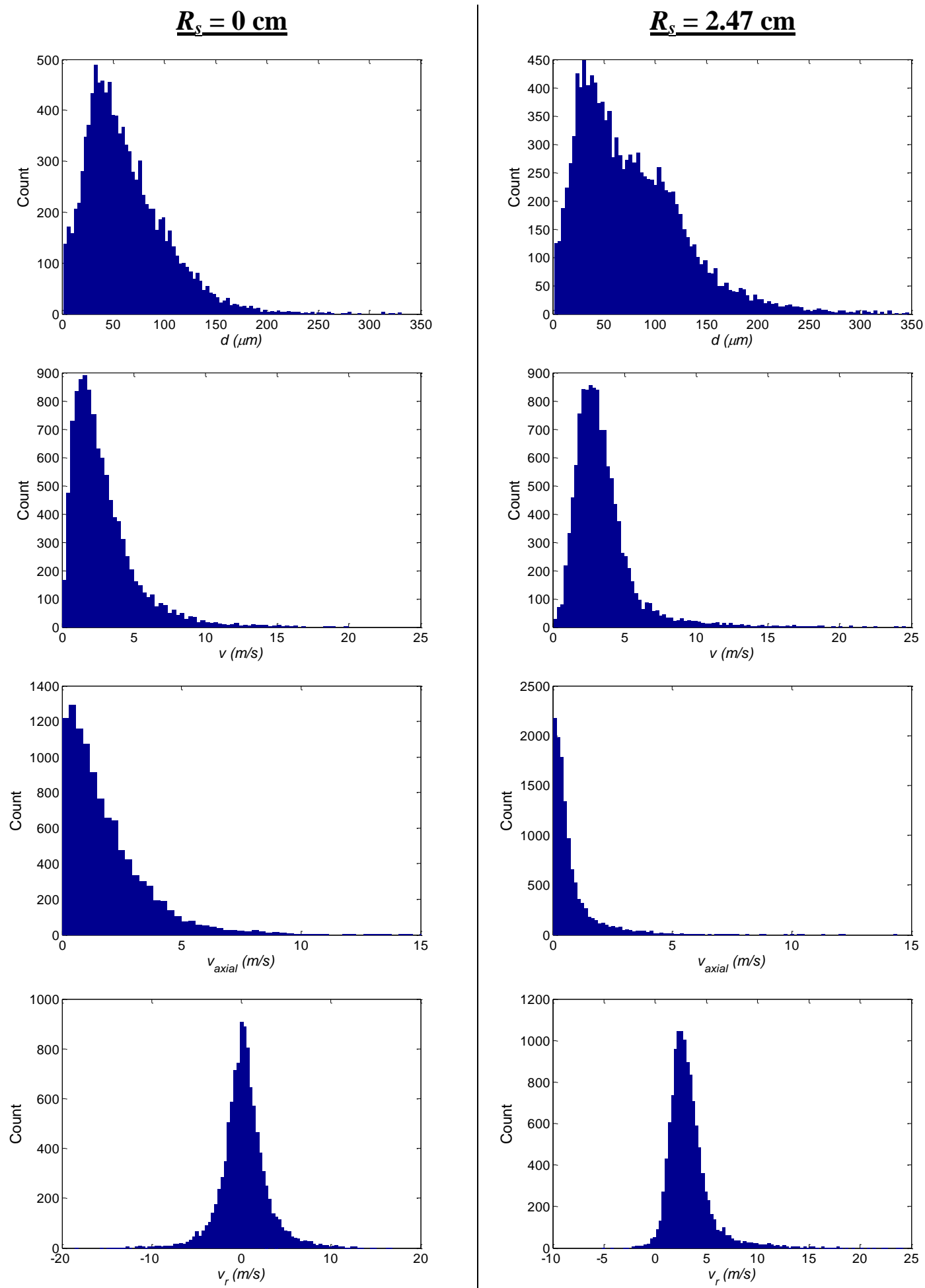

a)

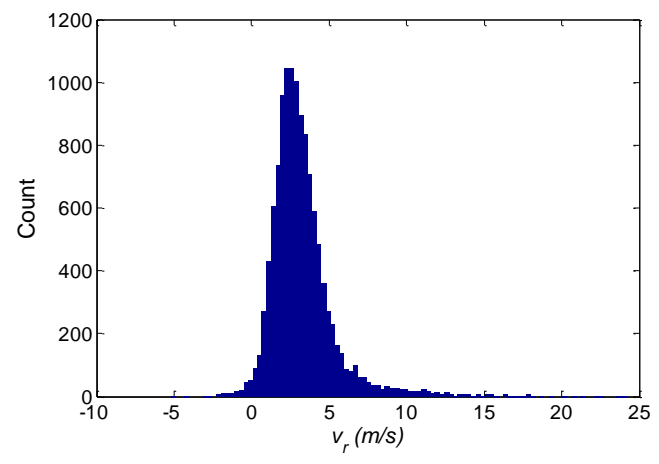

b)

Figure C.12: Histograms of the absolute splashed droplet data for $1.38 \mathrm{bar}, 29.5 \mathrm{~L} / \mathrm{hr}$ at $z=$ $3.65 \mathrm{~cm}$ at a single point. a) $R_{s}=0 \mathrm{~cm}$. b) $R_{s}=2.47 \mathrm{~cm}$. 


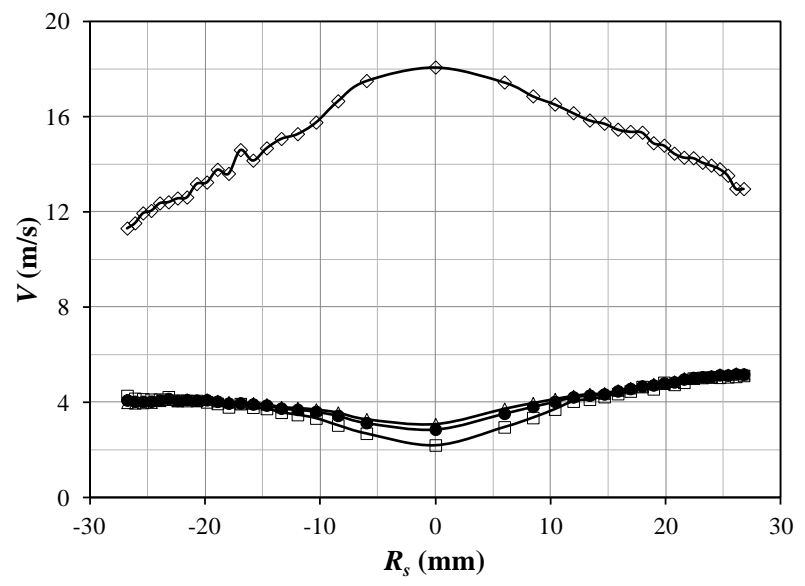

Figure C.13: Droplet velocity magnitude of the spray, ejected, return, and absolute splash data with the impact surface installed for a flow condition of $4.14 \mathrm{bar}, 54.5 \mathrm{~L} / \mathrm{hr}$ at a constant $z=$ $36.5 \mathrm{~mm}$. $\checkmark$ Impinging spray droplets, $\triangleleft$ ejected splashed droplets, $\neg \square$ - returning splashed droplets, - - absolute splashed droplets.

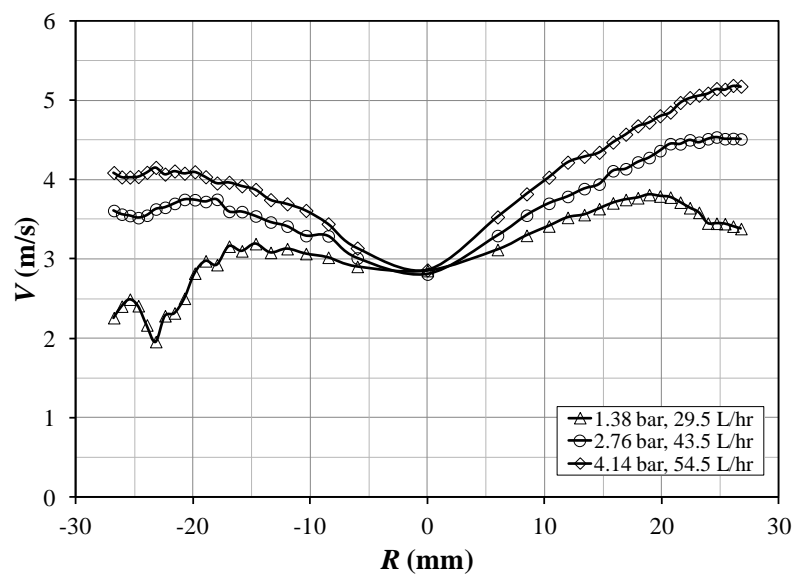

Figure C.14: Absolute splashed droplet mean velocity magnitude profile for varying pressures at a constant $z=36.5 \mathrm{~mm}$.

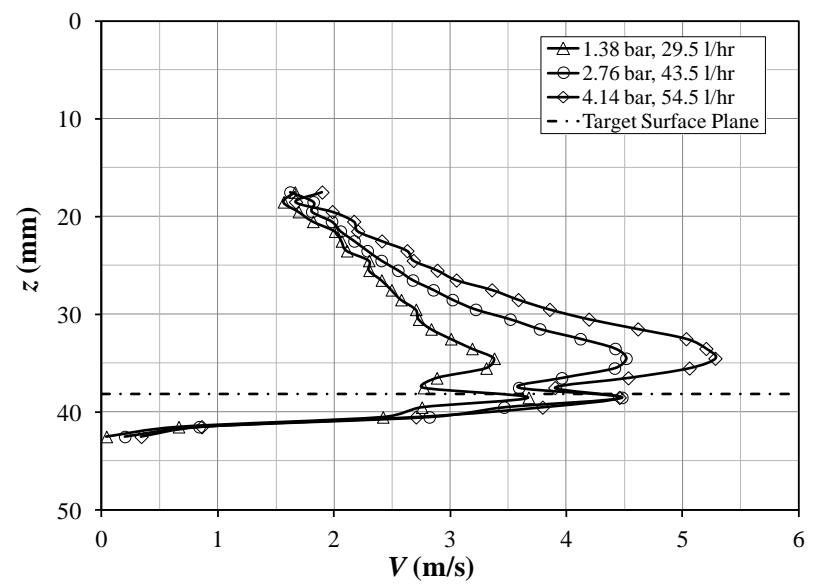

Figure C.15: PDPA data of the mean velocity magnitude versus $z$ at $R_{s}= \pm 2.68 \mathrm{~cm}$. 


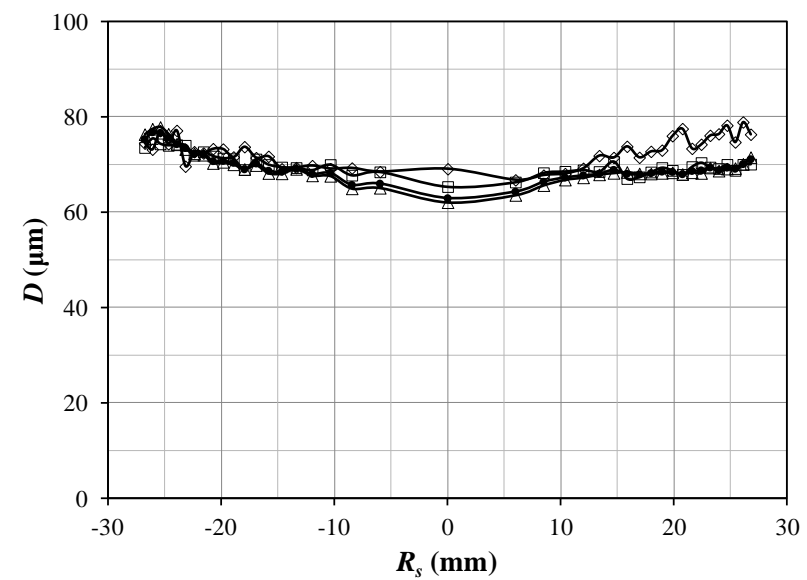

a)

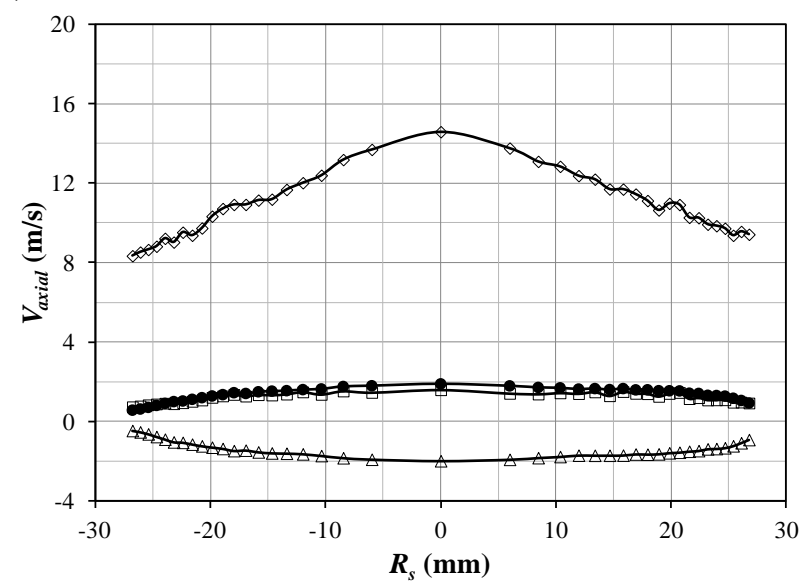

c)

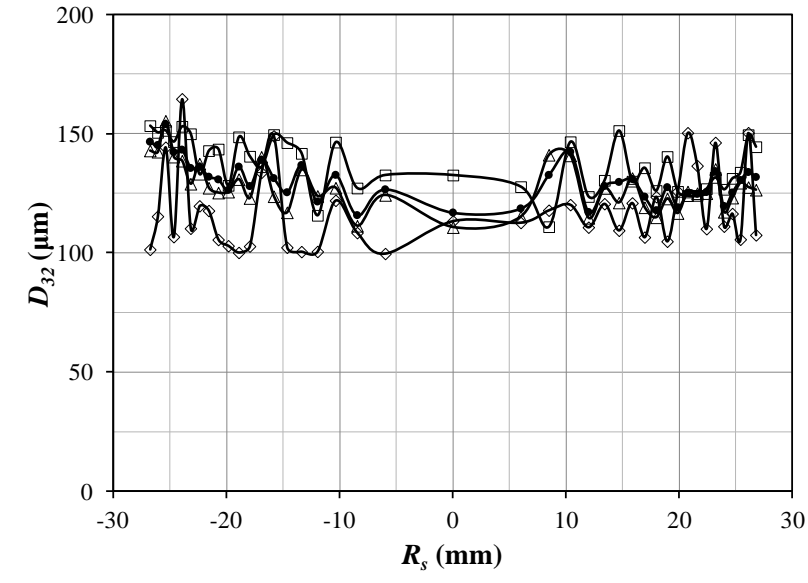

b)

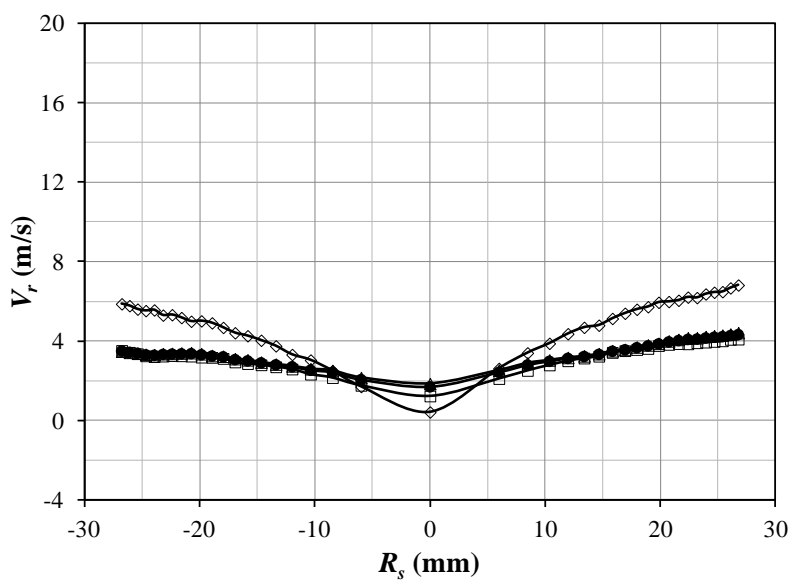

d)

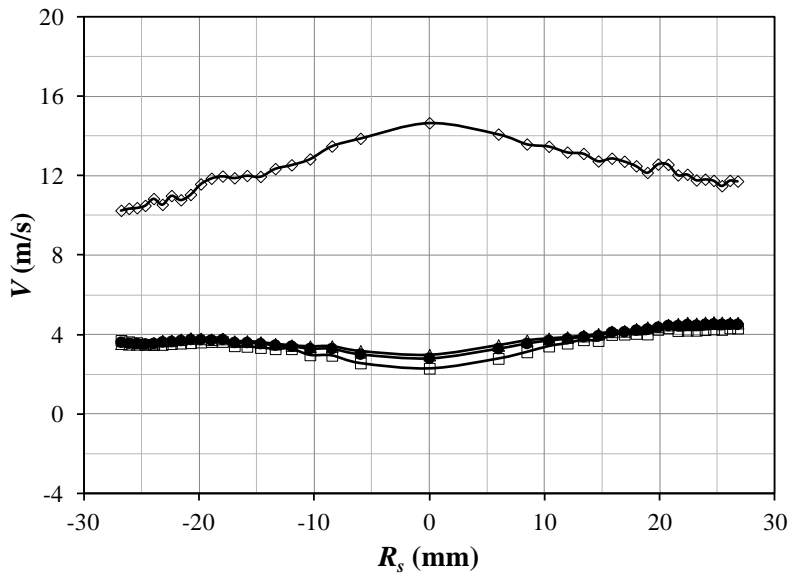

e)

Figure C.16: Comparison of the spray droplet characteristics with and without the impact surface installed a flow condition of $2.76 \mathrm{bar}, 43.5 \mathrm{~L} / \mathrm{hr}$ at a constant $z=36.5 \mathrm{~mm}$. a) Arithmetic mean diameter. b) Sauter mean diameter. c) Mean axial velocity. d) Mean radial velocity. e) Mean velocity magnitude. $\checkmark$ Impinging spray droplets, $\checkmark$ ejected splashed droplets, $-\square$ - returning splashed droplets, - - absolute splashed droplets. 


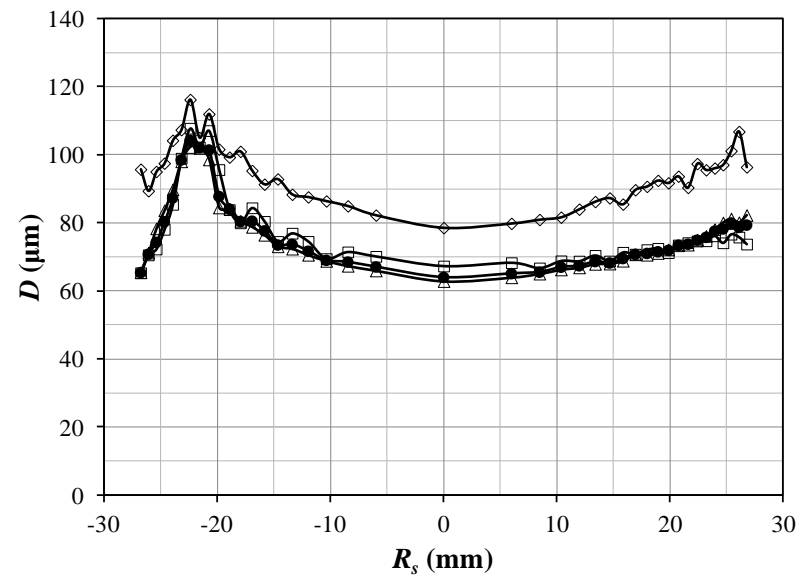

a)

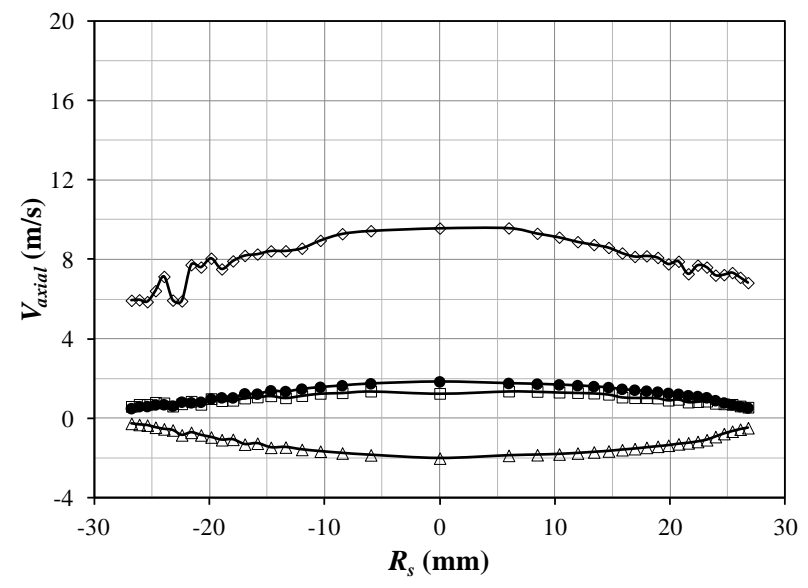

c)

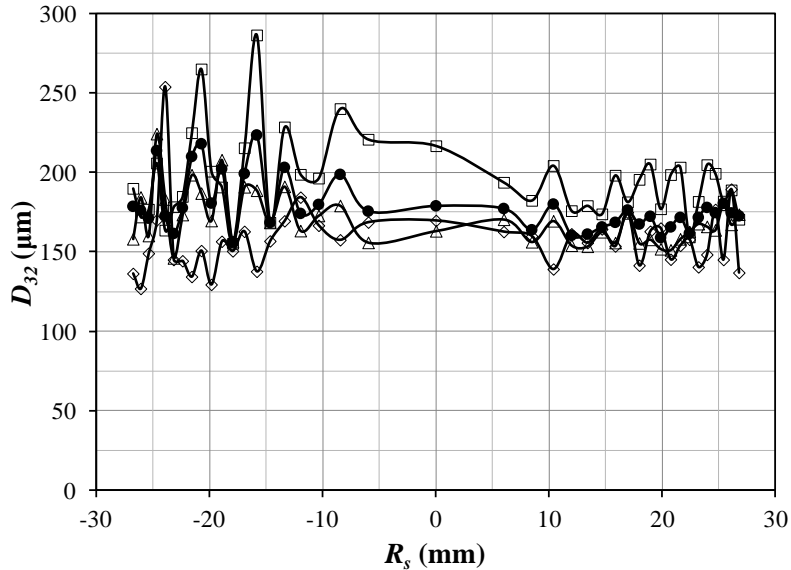

b)

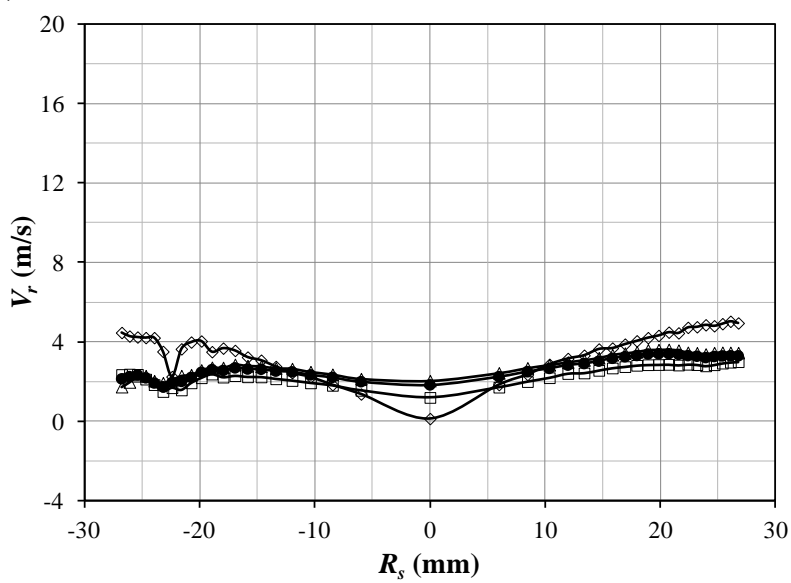

d)

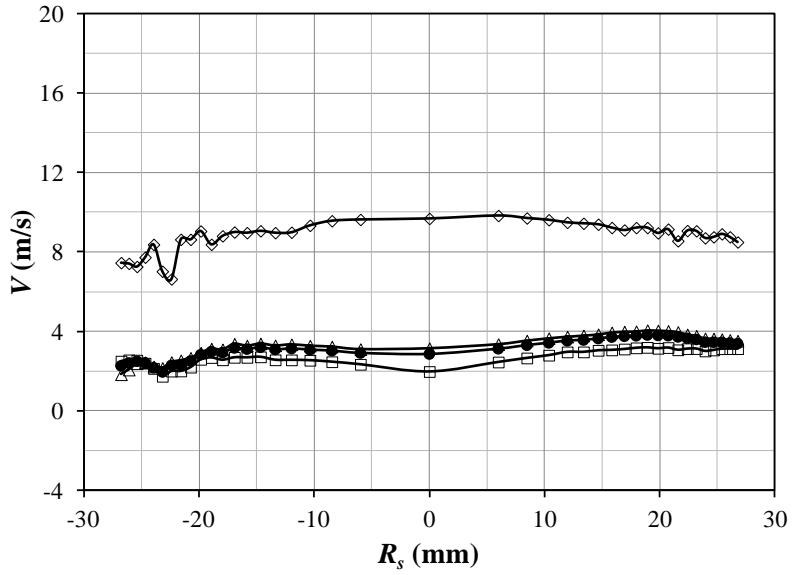

e)

Figure C.17: Comparison of the spray droplet characteristics with and without the impact surface installed a flow condition of $1.38 \mathrm{bar}, 29.5 \mathrm{~L} / \mathrm{hr}$ at a constant $z=36.5 \mathrm{~mm}$. a) Arithmetic mean diameter. b) Sauter mean diameter. c) Mean axial velocity. d) Mean radial velocity. e) Mean velocity magnitude. $\checkmark$ Impinging spray droplets, $\triangleleft-$ ejected splashed droplets, $-\square$ - returning splashed droplets, - - absolute splashed droplets. 


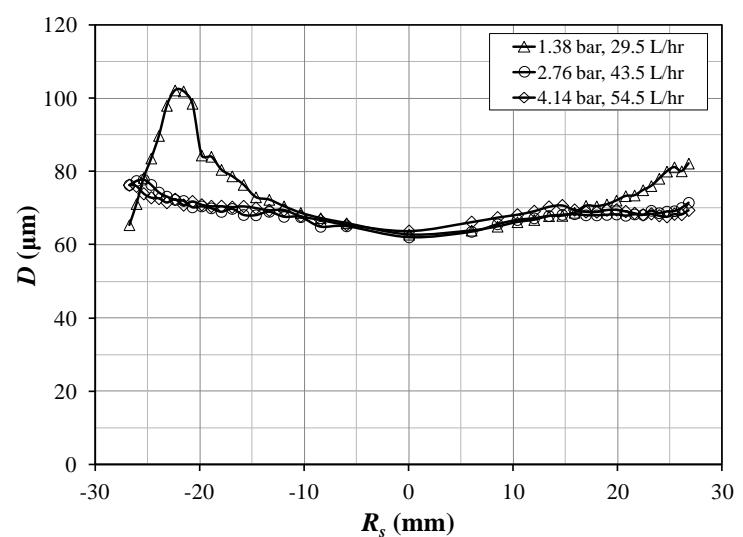

a)

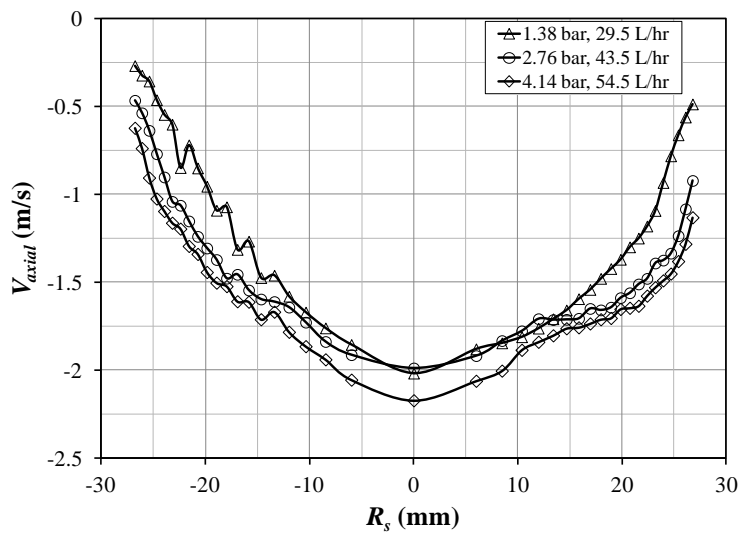

c)

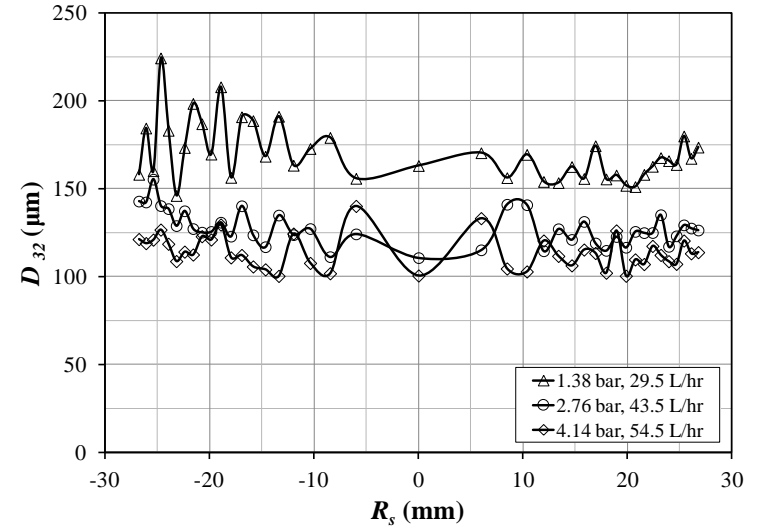

b)

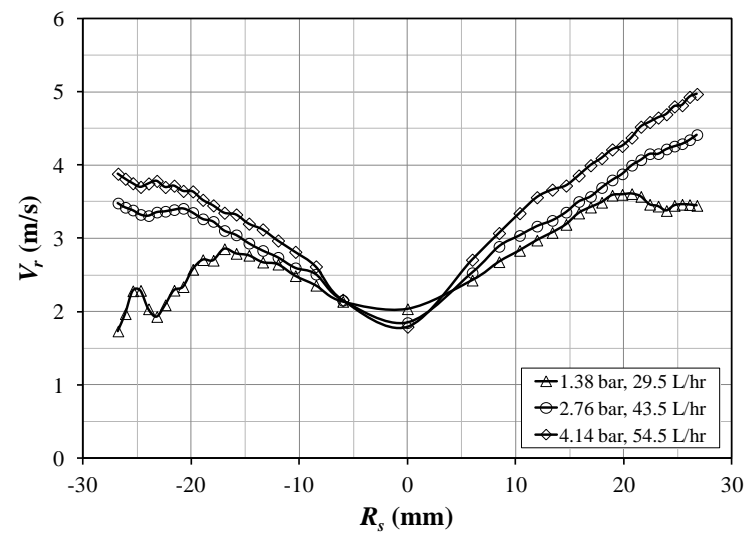

d)

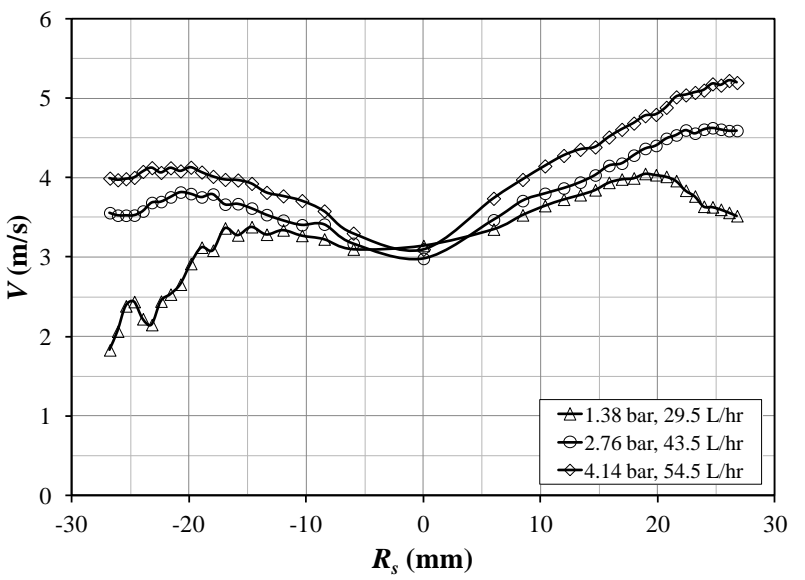

e)

Figure C.18: Ejected splashed droplet characteristics at varying pressures at a constant $z=$ $36.5 \mathrm{~mm}$. a) Arithmetic mean droplet diameter. b) Sauter mean droplet diameter. c) Mean axial velocity. d) Mean radial velocity. e) Mean velocity magnitude. 


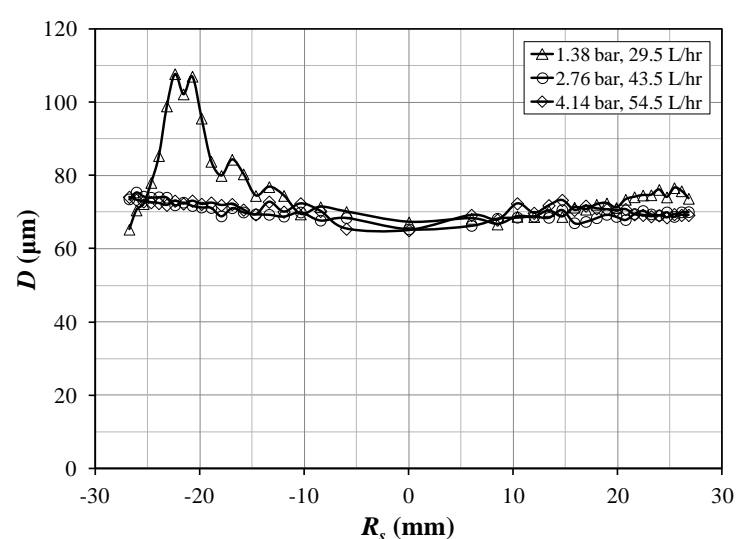

a)

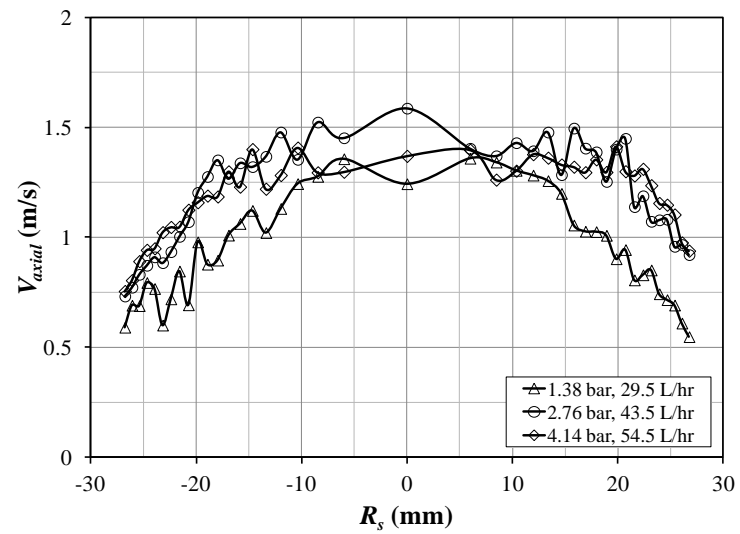

c)

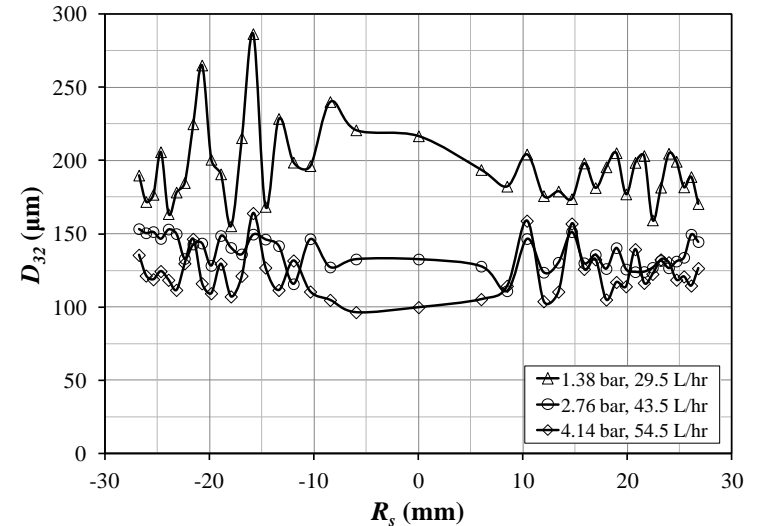

b)

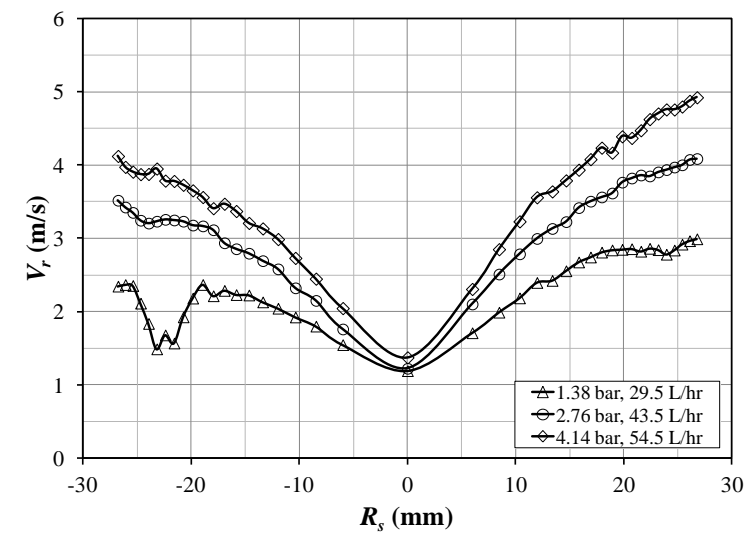

d)

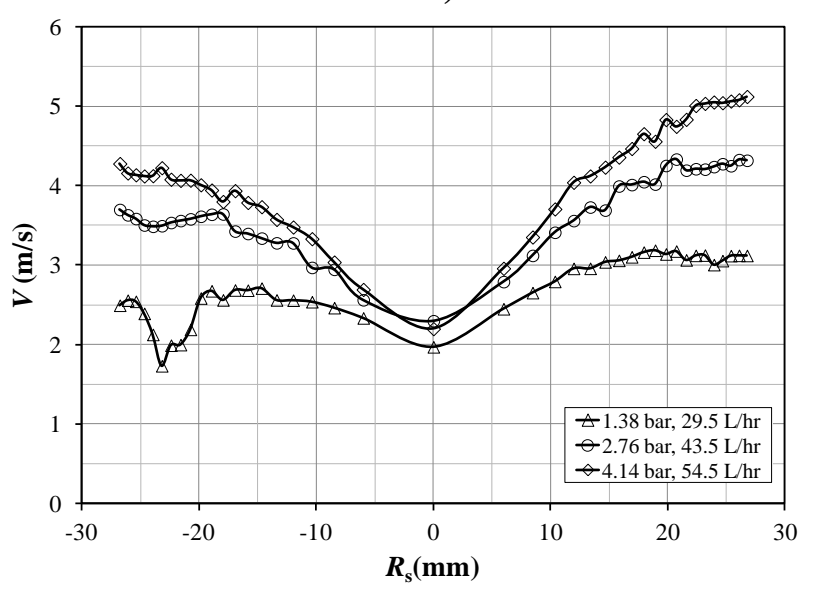

e)

Figure C.19: Returning splashed droplet characteristics at varying pressures at a constant $z=$ $36.5 \mathrm{~mm}$. a) Arithmetic mean droplet diameter. b) Sauter mean droplet diameter. c) Mean axial velocity. d) Mean radial velocity. e) Mean velocity magnitude. 


\section{Appendix D: Glycerol-Water Solution Methodology AND CHR THICKNESS VALIDATION}

Appendix D contains further explanation of how the glycerol-water solution by mass was computed to match the single drop and spray dimensionless numbers ( $W e$ and $R e$ ). Also, included is a comparison of the of a static liquid layer thickness measured manually from the videos and by the CHR sensor. 
The correct glycerol-water mass fraction, calculated from Equation D.1, was solved iteratively starting with the target Weber number. The process for computing the viscosity and density of different glycerol-water concentrations follows procedure described by Cheng (2008). The corresponding volume fraction (Equation D.2) was initially computed for pure water (0\% volume fraction of glycerol in water). The "Goal Seek" Excel function was used to iterate the necessary volume fraction of glycerol to produce the proper Reynolds number. Then the droplet velocity was iterated using same function to match the Weber number with the target value again. The Droplet velocity and volume fraction of the solution was iterated until their respective errors converged. Surface tensions were computed from Equation D.3, which was extracted from a data base (Glycerol Producer's Society, 1963) plotted versus mass fraction in Figure D.1.

Equation D.1 was used to compute the mass percentage of the water-glycerol solution.

$$
C_{m}=\frac{m_{G}}{m_{G}+m_{W}}
$$

Equation D.2 was used to compute the volume percentage of the water-glycerol solution.

$$
\Phi_{m}=\frac{V o l_{G}}{V o l_{G}+V o l_{W}}
$$

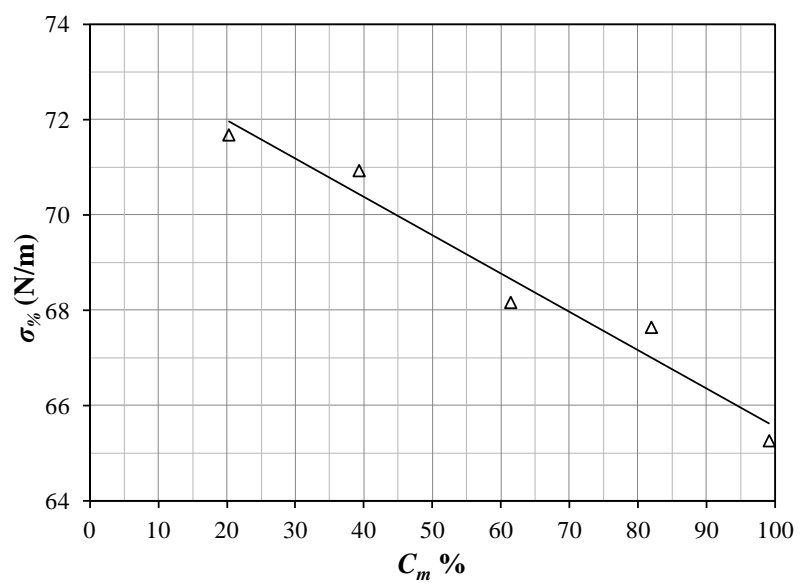

Figure D.1: Surface tension versus glycerol-water solution percent concentration by mass for $T=20^{\circ}$.

Surface tension for the glycerol-water mixture where computed using the linear curve fit from Figure D.1 given by Equation D.3.

$$
\sigma_{\text {sol }}=-0.0804 C_{m}+73.591
$$




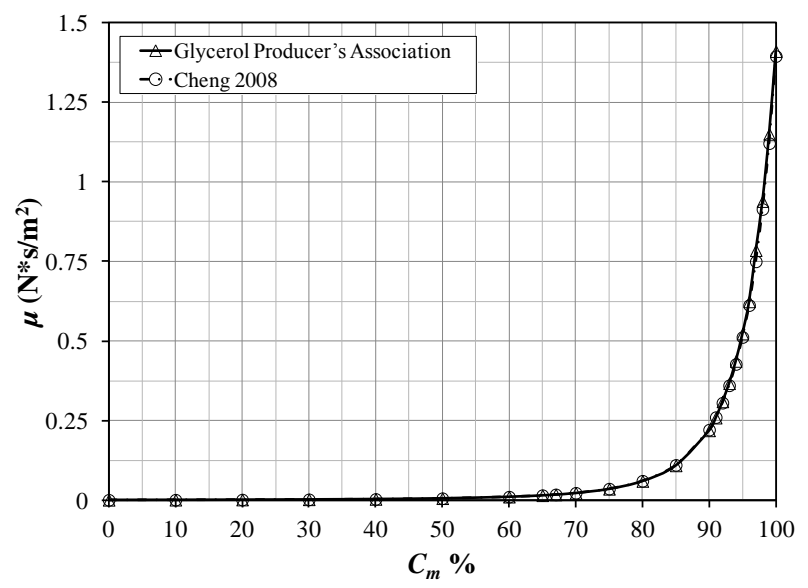

Figure D.2: Viscosity prediction from Cheng (2008) compared with data from Glycerol Producer's Association (1963).

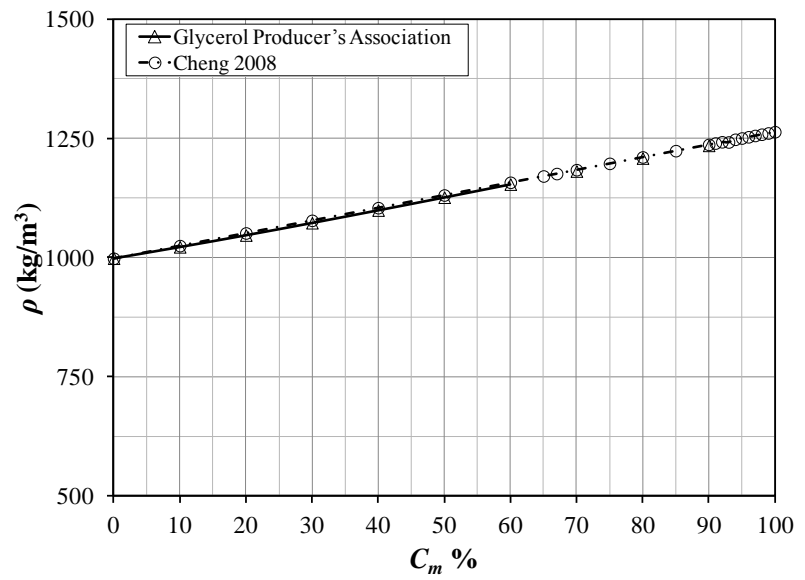

Figure D.3: Density prediction from Cheng (2008) compared with data from Glycerol Producer's Association (1963).

Equations D.4 - D.6 are curve fits of measured refractive indexes and were used to compute the refractive index of the water-glycerol solution based on percent mass (Glycerol Producer's Association, 1963).

$$
\begin{array}{cc}
\eta_{\text {sol }}=1.3303+0.001124 C_{m}+0.00000605 C_{m}^{2}-0.000000555 C_{m}^{2}, & \mathrm{D} .4 \\
\text { for } C_{m}: 0-44 \% & \mathrm{D} .5 \\
\eta_{\text {sol }}=0.00149 C_{m}+1.32359, \text { for } C_{m}: 45-79 \% & \\
\eta_{\text {sol }}=0.90799+0.0154 C_{m}-0.000155 C_{m}^{2}+0.000000576 C_{m}^{3}, & \text { D.6 } \\
\text { for } C_{m}: 80-100 \% &
\end{array}
$$




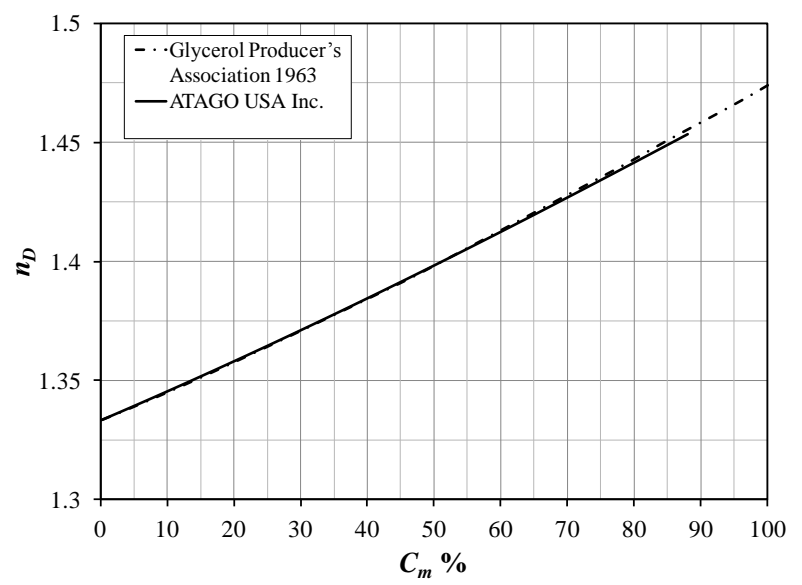

Figure D.4: Comparison of the predictive capability of curve fits from Glycerol Producers Association (1963) with available refractive indices of glycerol-water mixtures.

Undisturbed static film thickness measurements were performed by manually measuring an image in the Photron FASTCAM Viewer software. This was compared with the CHR sensor readings using the refractive index computed for glycerol-water solution of $46.2 \%$ by mass using Equation D.5. Figure D.5 shows that the distance was measured to the bottom of the wall meniscus.

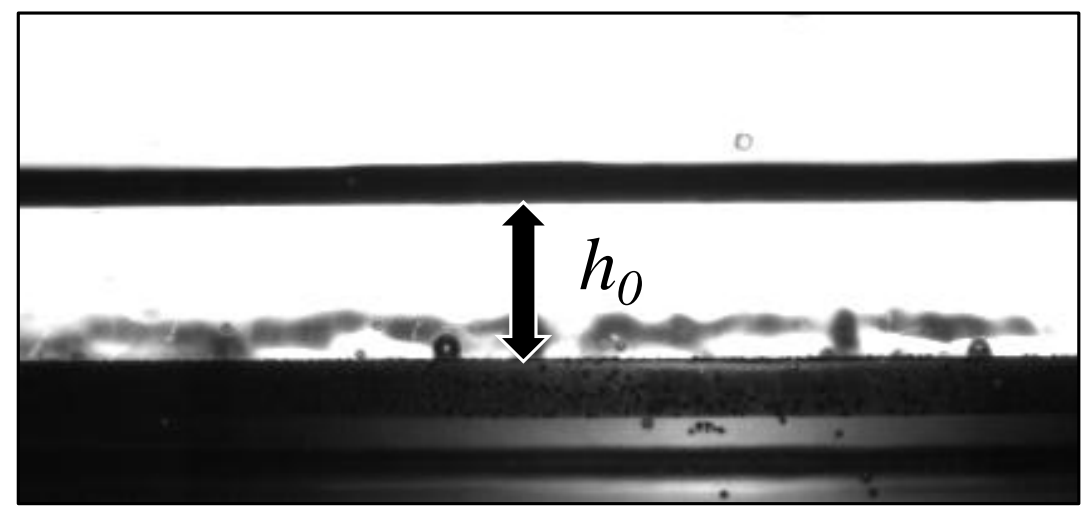

Figure D.5: Initial film thickness manual measurements.

Table D.1 lists the measured liquid film thickness computed from the respective video analysis and CHR sensor readings. Indexes of refraction were back computed using Equation 4.1, and the variance was compared with the refractive index used for the CHR sensor. The CHR refractive index was 1.39 computed for a $46.2 \%$ water-glycerol solution using Equation D.5. The variance was negligible thus the curve fits from Equations D.4 - D.6 are acceptable. The variance that does occur can be contributed to the $50.7 \mu \mathrm{m}$ pixel resolution in the video analysis. 
Table D.1: Target liquid film thickness measured by the CHR sensor and by video. Included is the refractive index calculated by the video analysis for a glycerol-water mixture of $46.2 \%$.

\begin{tabular}{|c|c|c|c|c|}
\hline \multicolumn{2}{|c|}{ CHR Sensor } & \multicolumn{2}{c|}{ Video Analysis } & \multicolumn{1}{c|}{} \\
\hline $\boldsymbol{h}(\boldsymbol{\mu m})$ & $\boldsymbol{h}_{\boldsymbol{0}}(\boldsymbol{\mu m})$ & $\boldsymbol{h}_{\boldsymbol{0}}(\boldsymbol{\mu m})$ & $\boldsymbol{\eta}$ & $\boldsymbol{c}_{\boldsymbol{v}}(\boldsymbol{\eta})$ \\
\hline 2,304 & 3075 & 3,093 & 1.40 & $\pm 0.40 \%$ \\
\hline 2,254 & 3005 & 3,042 & 1.38 & $\pm 0.77 \%$ \\
\hline 2,141 & 2848 & 2,890 & 1.41 & $\pm 1.0 \%$ \\
\hline 2,315 & 3104 & 3,093 & 1.39 & $\pm 0.26 \%$ \\
\hline 2,310 & 3083 & 3,093 & 1.40 & $\pm 0.23 \%$ \\
\hline
\end{tabular}




\section{Appendix E: $\quad$ AdDitional Single Droplet Sub-Cavity ReSUlts}

Contained herein are the single drop sub-cavity results for Cases 1 - 5 not presented in Chapter 7. Included are the respective sub-cavity radius and centerline sub-cavity liquid film thickness histories. Also included are the average measured droplet characteristics used to compute the dimensionless numbers in Table 7.1. 
Table E.1: Measured sub-cavity single drop parameters and standard deviations for Cases 1 5 for each $h_{0}{ }^{*}$.

\begin{tabular}{|c|c|c|c|c|c|c|c|}
\hline Case & $\boldsymbol{h}_{\boldsymbol{0}}{ }^{*}$ & $\boldsymbol{h}_{\boldsymbol{0}}(\boldsymbol{\mu m})$ & $\boldsymbol{D}(\mathbf{m m})$ & $\boldsymbol{V}(\mathbf{m} / \mathbf{s})$ & $\boldsymbol{s t d}(\boldsymbol{d})(\mathbf{m m})$ & $\boldsymbol{s t d}(\boldsymbol{v})(\mathbf{m} / \mathbf{s})$ & $\boldsymbol{s t d}(\boldsymbol{x c})(\mathbf{m m})$ \\
\hline \multirow{4}{*}{1} & 1.0 & 3,020 & 3.03 & 1.73 & 0.0689 & 0.0115 & 0.0931 \\
\cline { 2 - 8 } & 0.5 & 1,530 & 3.06 & 1.73 & 0.0326 & 0.0244 & 0.0551 \\
\cline { 2 - 8 } & 0.2 & 724 & 3.03 & 1.67 & 0.0341 & 0.0462 & 0.0939 \\
\hline \multirow{4}{*}{2} & 1.0 & 3,500 & 3.47 & 2.72 & 0.0106 & 0.00460 & 0.0270 \\
\cline { 2 - 8 } & 0.5 & 1,760 & 3.47 & 2.73 & 0.0101 & 0.00370 & 0.0397 \\
\cline { 2 - 8 } & 0.2 & 714 & 3.48 & 2.72 & 0.00940 & 0.00790 & 0.0457 \\
\hline \multirow{4}{*}{3} & 1.0 & 3,010 & 3.09 & 3.55 & 0.0359 & 0.00520 & 0.0859 \\
\cline { 2 - 8 } & 0.5 & 1,550 & 3.08 & 3.55 & 0.0288 & 0.0128 & 0.0666 \\
\cline { 2 - 8 } & 0.2 & 606 & 3.11 & 3.66 & 0.0278 & 0.00578 & 0.430 \\
\hline \multirow{4}{*}{4} & 0.9 & 3,120 & 3.49 & 3.69 & 0.00633 & 0.00676 & 0.0770 \\
\cline { 2 - 8 } & 0.5 & 1,730 & 3.47 & 3.72 & 0.0170 & 0.00925 & 0.0621 \\
\cline { 2 - 8 } & 0.2 & 695 & 3.46 & 3.75 & 0.0213 & 0.00892 & 0.0661 \\
\hline \multirow{3}{*}{5} & 0.9 & 3,160 & 3.52 & 4.20 & 0.00682 & 0.00562 & 0.0770 \\
\cline { 2 - 8 } & 0.5 & 1,750 & 3.54 & 4.16 & 0.00925 & 0.00662 & 0.0689 \\
\cline { 2 - 7 } & 0.2 & 707 & 3.52 & 4.18 & 0.0350 & 0.00456 & 0.0950 \\
\hline
\end{tabular}

Table E.2: Average ambient conditions and respective variance recorded during the Single drop experiments for Cases $1-5$ for each $\boldsymbol{h}_{0}{ }^{*}$.

\begin{tabular}{|c|c|c|c|c|c|c|c|}
\hline Case & $\boldsymbol{h}_{\boldsymbol{0}}{ }^{*}$ & $\boldsymbol{T}\left({ }^{\circ} \mathbf{C}\right)$ & $\mathbf{A b s}(\boldsymbol{P})(\mathbf{i n H g})$ & $\boldsymbol{R H}$ & $\boldsymbol{s t d}(\boldsymbol{T}) \mathbf{( m m})$ & $\boldsymbol{s t d}(\boldsymbol{A b s}(\boldsymbol{P}))(\mathbf{m} / \mathbf{s})$ & $\boldsymbol{s t d}(\boldsymbol{R H})$ \\
\hline \multirow{3}{*}{1} & 1.0 & N/A & N/A & N/A & N/A & N/A & N/A \\
\cline { 2 - 8 } & 0.5 & 22.0 & 28.6 & $47.6 \%$ & 0.109 & 0.0209 & $1.77 \%$ \\
\cline { 2 - 8 } & 0.2 & 20.7 & 27.3 & $41.6 \%$ & 0.115 & 0.0154 & $1.07 \%$ \\
\hline \multirow{3}{*}{2} & 1.0 & 21.4 & 29.1 & $28.4 \%$ & 0.311 & 0.0140 & $0.505 \%$ \\
\cline { 2 - 8 } & 0.5 & 21.6 & 29.1 & $27.3 \%$ & 0.302 & 0.0156 & $0.480 \%$ \\
\cline { 2 - 8 } & 0.2 & 21.8 & 28.9 & $43.6 \%$ & 0.298 & 0.0104 & $0.756 \%$ \\
\hline \multirow{3}{*}{3} & 1.0 & 22.0 & 29.1 & $<20.0 \%$ & 0.255 & 0.314 & N/A \\
\cline { 2 - 8 } & 0.5 & 21.7 & 29.3 & $<20.0 \%$ & 0.380 & 0.242 & N/A \\
\cline { 2 - 8 } & 0.2 & 21.7 & 28.6 & $46.1 \%$ & 0.250 & 0.0722 & $1.290 \%$ \\
\hline \multirow{3}{*}{4} & 0.9 & 21.4 & 29.1 & $36.5 \%$ & 0.132 & 0.0189 & $0.0519 \%$ \\
\cline { 2 - 8 } & 0.5 & 21.5 & 30.0 & $34.5 \%$ & 0.210 & 0.0132 & $0.00660 \%$ \\
\cline { 2 - 8 } & 0.2 & 21.4 & 29.9 & $27.0 \%$ & 0.302 & 0.00832 & $0.000 \%$ \\
\hline \multirow{3}{*}{5} & 0.9 & 21.8 & 28.8 & $44.3 \%$ & 0.163 & 0.0249 & $0.0467 \%$ \\
\cline { 2 - 8 } & 0.5 & 21.7 & 28.8 & $45.5 \%$ & 0.262 & 0.000 & $0.522 \%$ \\
\cline { 2 - 8 } & 0.2 & 21.8 & 28.8 & $44.7 \%$ & 0.163 & 0.0767 & $0.467 \%$ \\
\hline
\end{tabular}




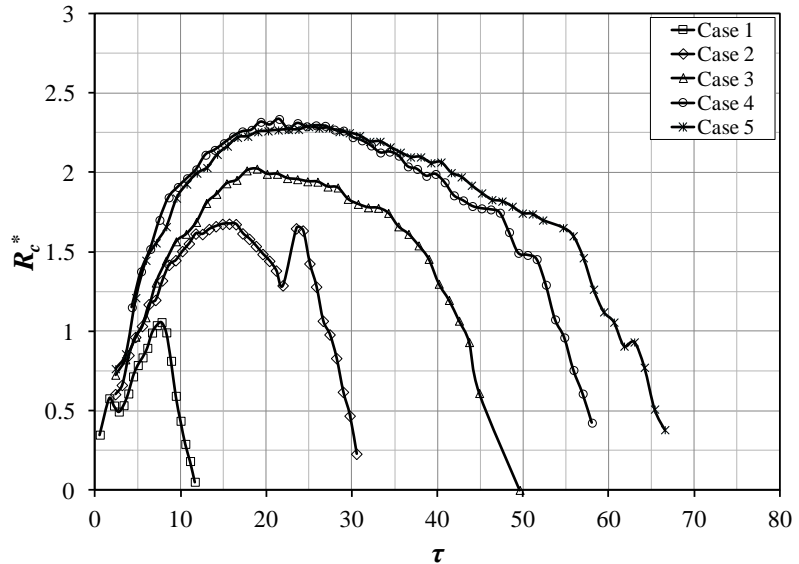

a)

Figure E.1: Sub-cavity radius histories varying for Cases $1-5$ at $h_{0}{ }^{*}=1.0\left(h_{0}{ }^{*}=0.9\right.$ for Cases 4 and 5). a) Bottom-view results. b) Side-view results.

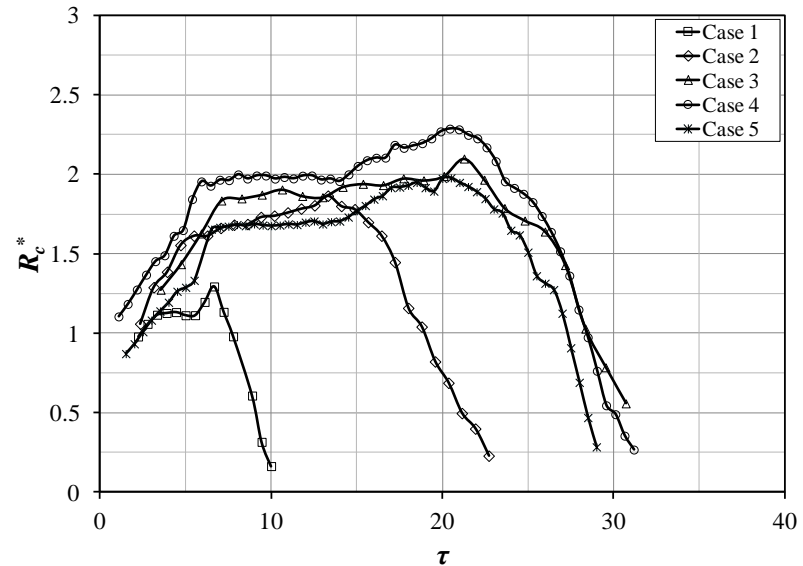

a)

Figure E.2: Sub-cavity radius histories varying for Cases $1-5$ at $\boldsymbol{h}_{0}{ }^{*}=0.2$. a) Bottom-view results. b) Side-view results.

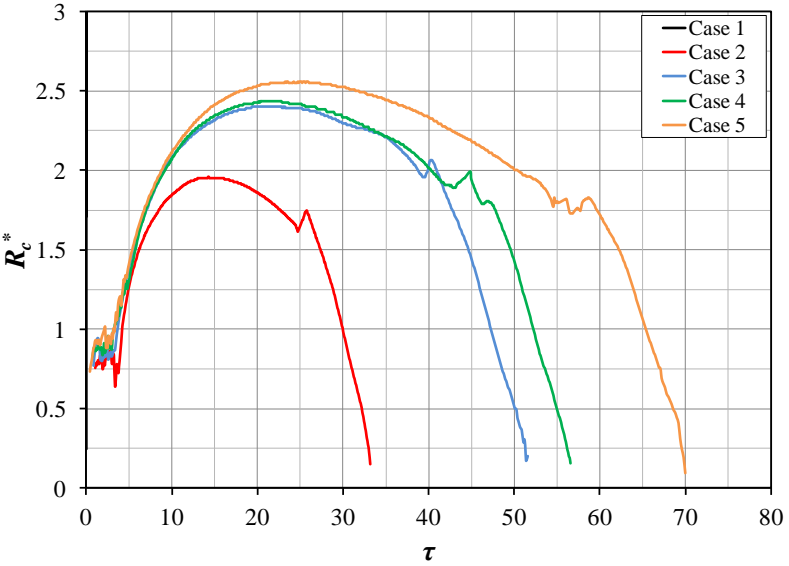

b)

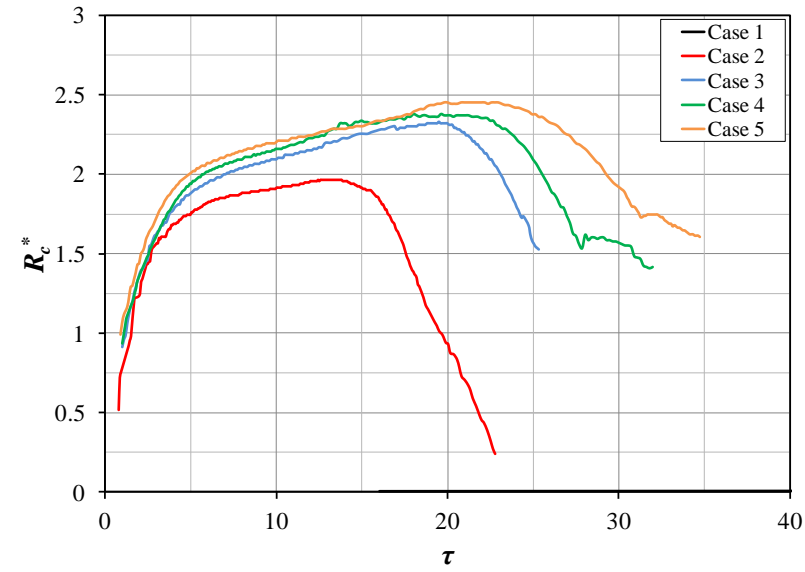

b) 


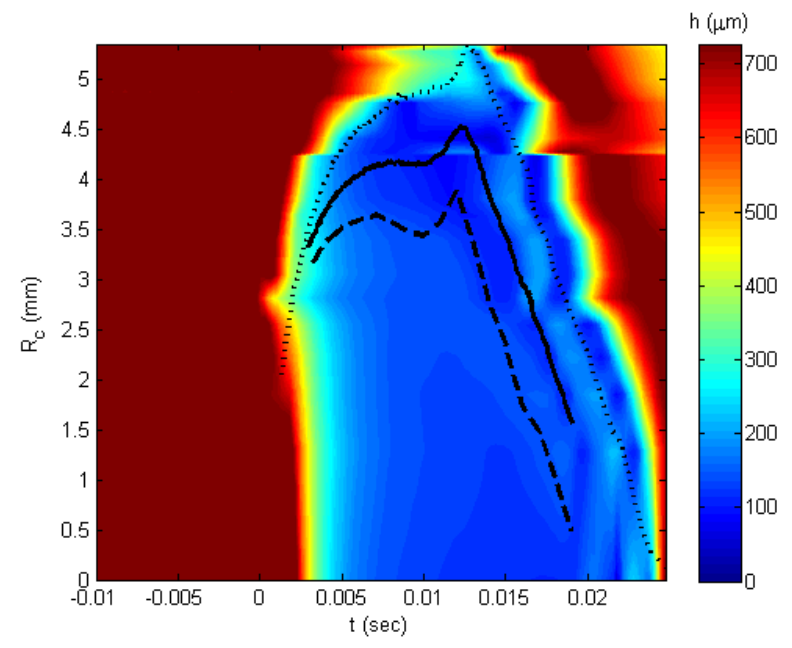

Figure E.3: Contour plot of with the bottom-view (dashed line), side-view (dotted line), and averaged (solid line) sub-cavity radius overlaid for Case $1, h_{0}{ }^{*}=0.2(W e=135, \operatorname{Re}=1,180, F r=$ 94.0).

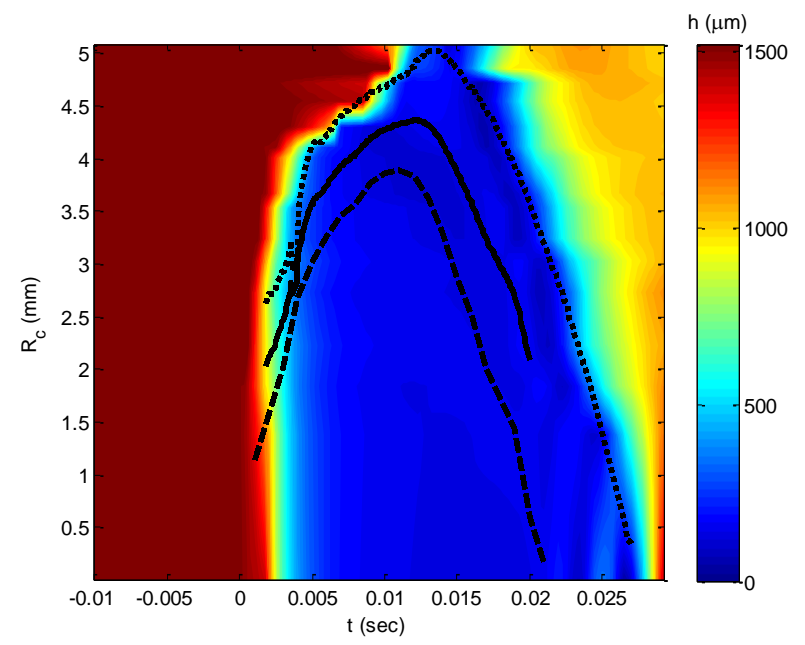

Figure E.4: Contour plot of with the bottom-view (dashed line), side-view (dotted line), and averaged (solid line) sub-cavity radius overlaid for Case $1, h_{0}{ }^{*}=0.5(W e=146, R e=1,290, F r=$ 99.0). 


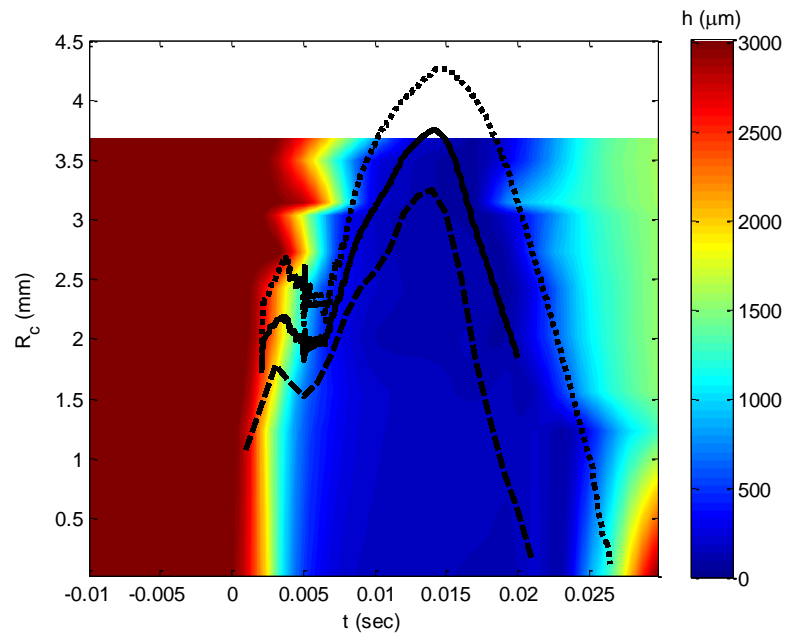

Figure E.5: Contour plot of with the bottom-view (dashed line), side-view (dotted line), and averaged (solid line) sub-cavity radius overlaid for Case $1, h_{0}{ }^{*}=1.0(W e=145, \operatorname{Re}=1,190, F r=$ 100).

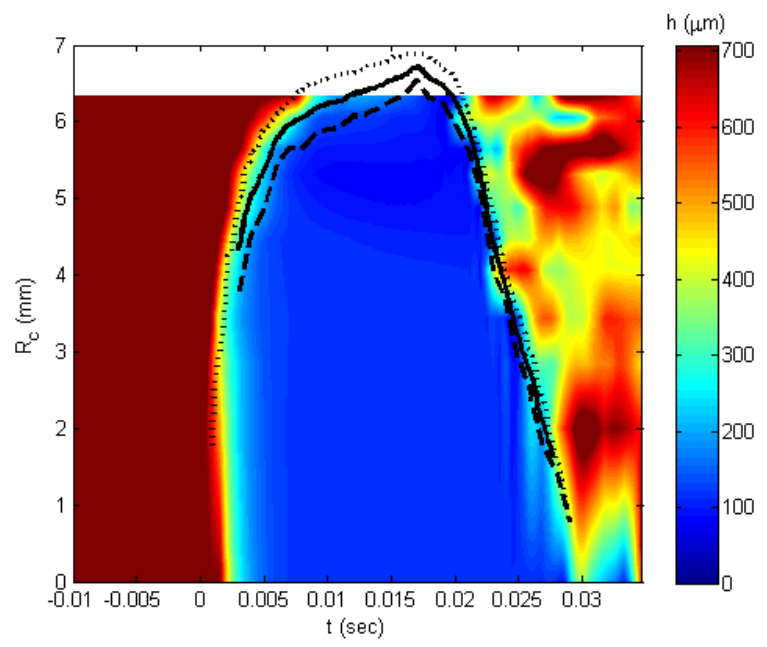

Figure E.6: Contour plot of with the bottom-view (dashed line), side-view (dotted line), and averaged (solid line) sub-cavity radius overlaid for Case $2, h_{0}{ }^{*}=0.2(W e=414, \operatorname{Re}=2,290, F r=$ 217). 


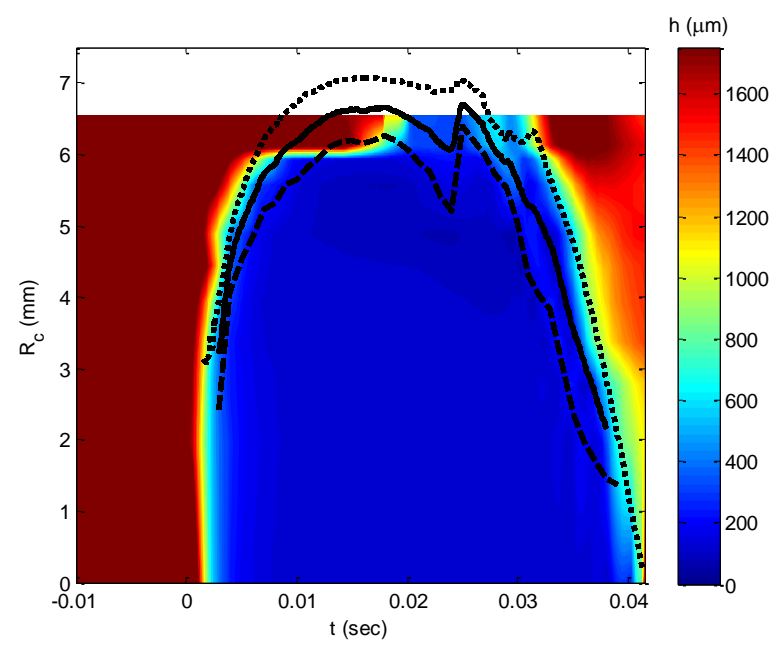

Figure E.7: Contour plot of with the bottom-view (dashed line), side-view (dotted line), and averaged (solid line) sub-cavity radius overlaid for Case $2, h_{0}{ }^{*}=0.5(W e=413, \operatorname{Re}=2,270, F r=$ 219).

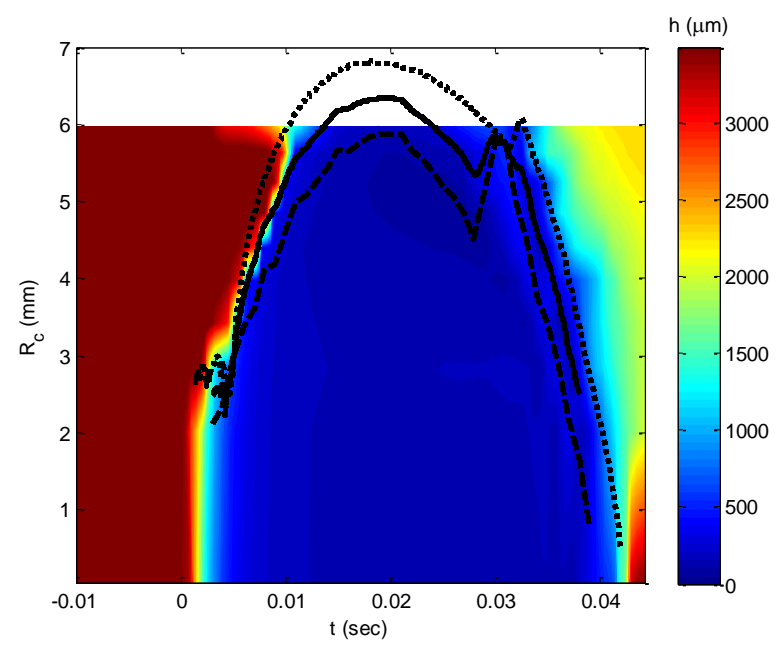

Figure E.8: Contour plot of with the bottom-view (dashed line), side-view (dotted line), and averaged (solid line) sub-cavity radius overlaid for Case $2, h_{0}{ }^{*}=1.0(W e=410, \operatorname{Re}=2,250, F r=$ 217). 


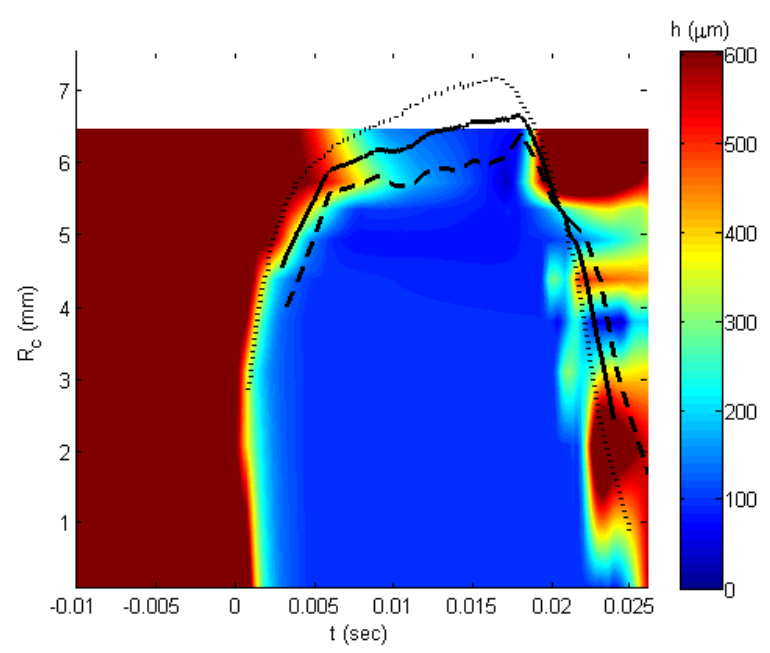

Figure E.9: Contour plot of with the bottom-view (dashed line), side-view (dotted line), and averaged (solid line) sub-cavity radius overlaid for Case $3, h_{0}{ }^{*}=0.2(W e=667, \operatorname{Re}=2,740, F r=$ 439).

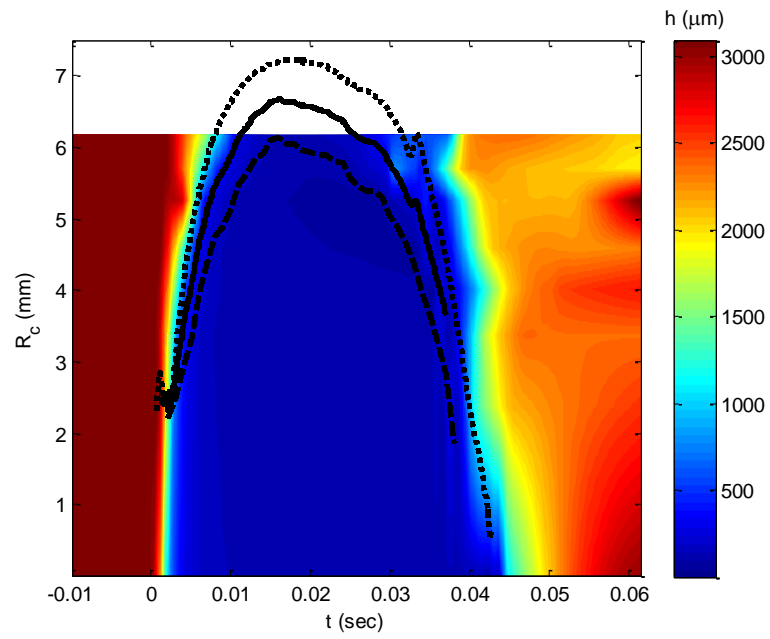

Figure E.10: Contour plot of with the bottom-view (dashed line), side-view (dotted line), and averaged (solid line) sub-cavity radius overlaid for Case $3, h_{0}{ }^{*}=1.0(W e=621, \operatorname{Re}=2,640, F r=$ 415). 


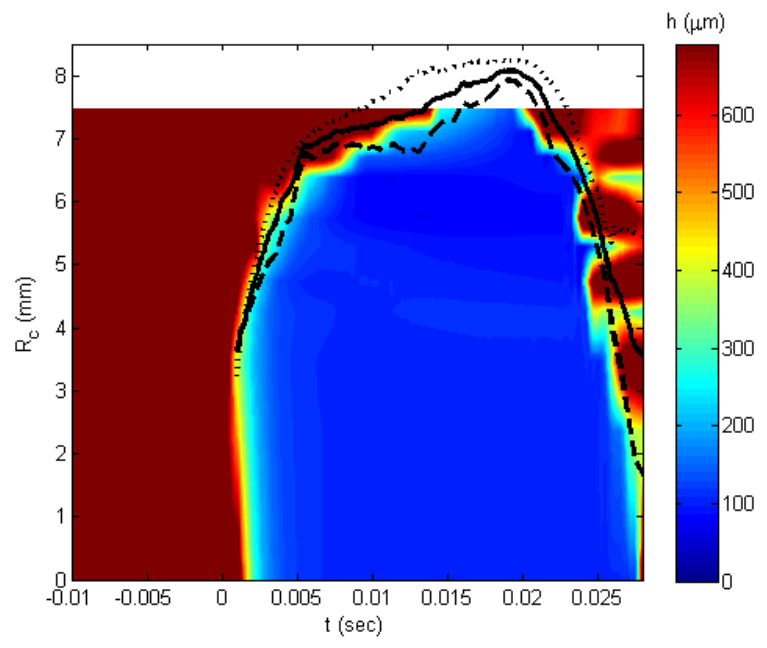

Figure E.11: Contour plot of with the bottom-view (dashed line), side-view (dotted line), and averaged (solid line) sub-cavity radius overlaid for Case $4, h_{0}{ }^{*}=0.2(W e=780, \operatorname{Re}=3,100, F r=$ 413).

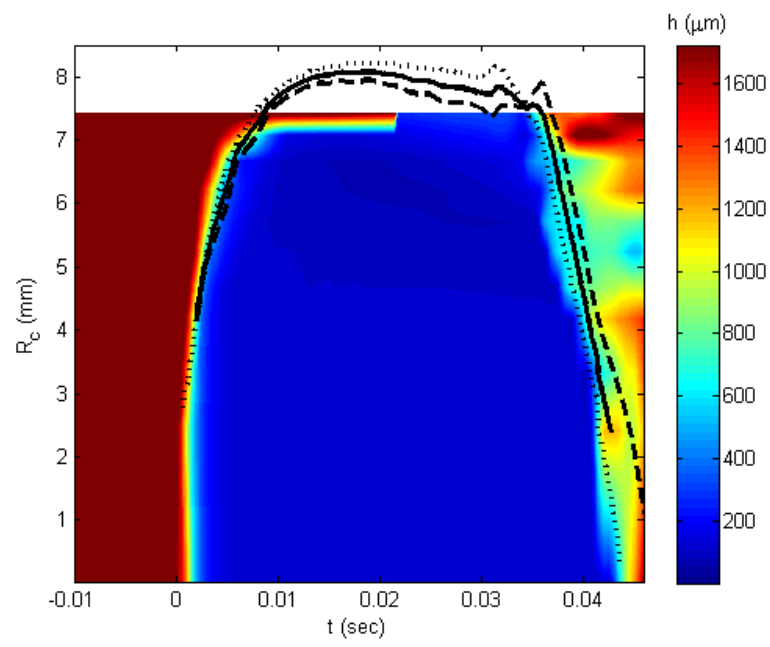

Figure E.12: Contour plot of with the bottom-view (dashed line), side-view (dotted line), and averaged (solid line) sub-cavity radius overlaid for Case $4, h_{0}{ }^{*}=0.5(W e=771, R e=3,090, F r=$ 407). 


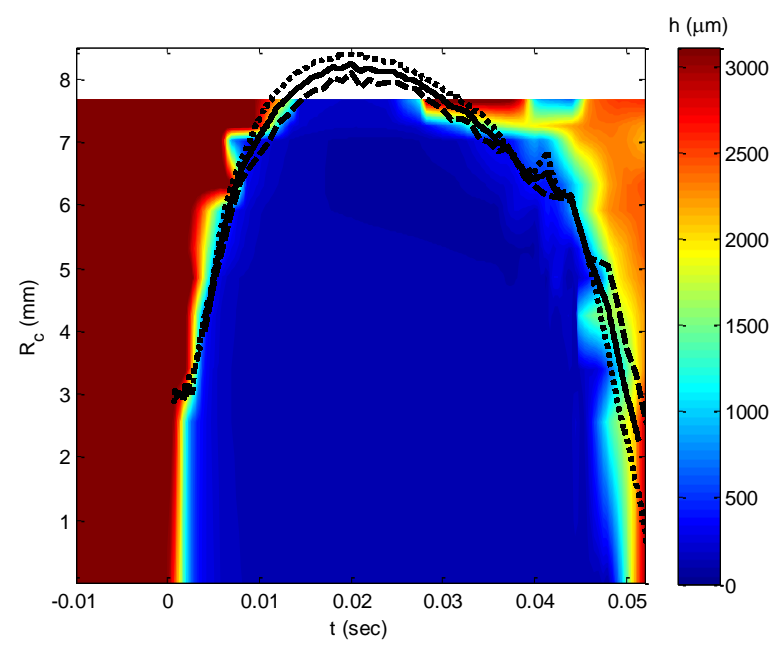

Figure E.13: Contour plot of with the bottom-view (dashed line), side-view (dotted line), and averaged (solid line) sub-cavity radius overlaid for Case $4, h_{0}{ }^{*}=0.9(W e=762, \operatorname{Re}=3,080, F r=$ 398).

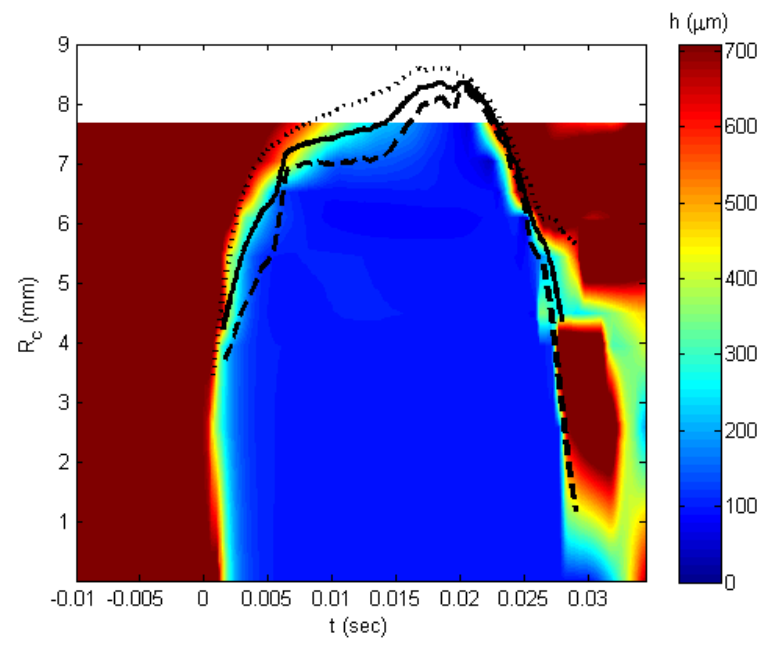

Figure E.14: Contour plot of with the bottom-view (dashed line), side-view (dotted line), and averaged (solid line) sub-cavity radius overlaid for Case $5, h_{0}{ }^{*}=0.2(W e=984, \operatorname{Re}=3,560, F r=$ 407). 


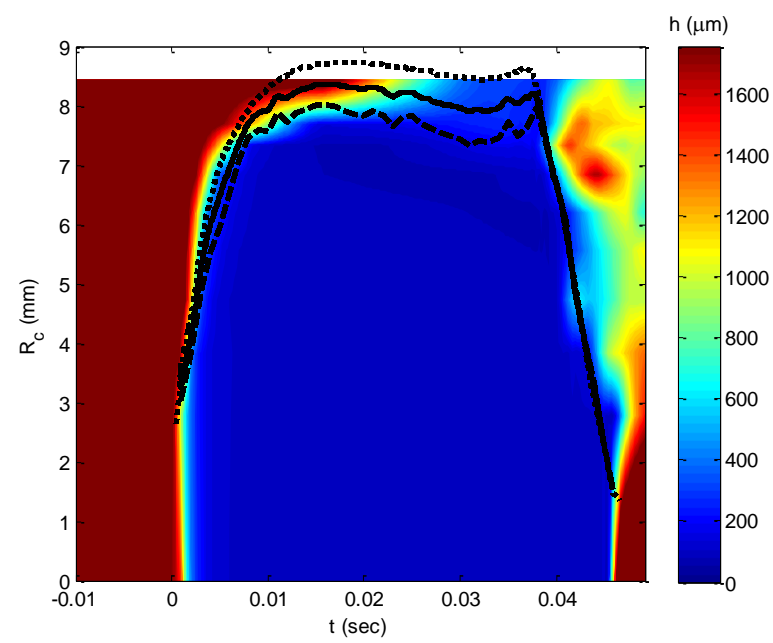

Figure E.15: Contour plot of with the bottom-view (dashed line), side-view (dotted line), and averaged (solid line) sub-cavity radius overlaid for Case $5, h_{0}{ }^{*}=0.5(W e=984, \operatorname{Re}=3,560, F r=$ 499).

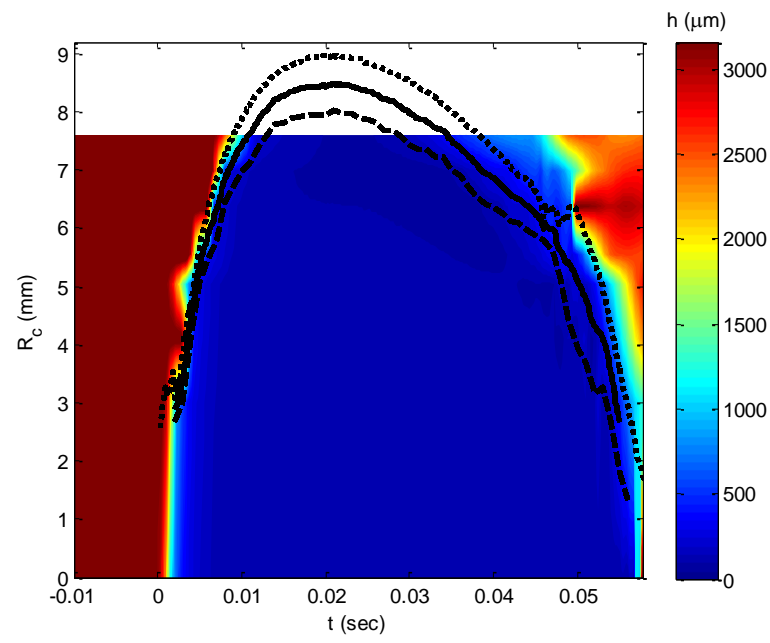

Figure E.16: Contour plot of with the bottom-view (dashed line), side-view (dotted line), and averaged (solid line) sub-cavity radius overlaid for Case $5, h_{0}{ }^{*}=0.9(W e=993, \operatorname{Re}=3,570, F r=$ 510). 


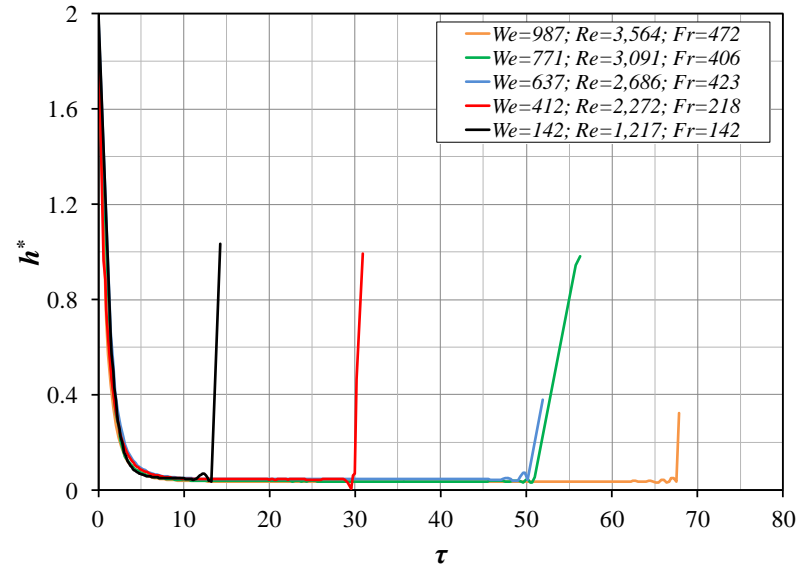

a)

Figure E.17: Dimensionless sub-cavity liquid film thickness $\left(h^{*}\right)$ histories for varying the initial droplet condition (We, Re, and $F r$ ) at a constant $h_{0}{ }^{*}$. a) $h_{0}{ }^{*}=1.0$ for Case $1-3$ and $h_{0}{ }^{*}=0.9$ for Cases 4 - 5. b) $h_{0}{ }^{*}=0.2$.
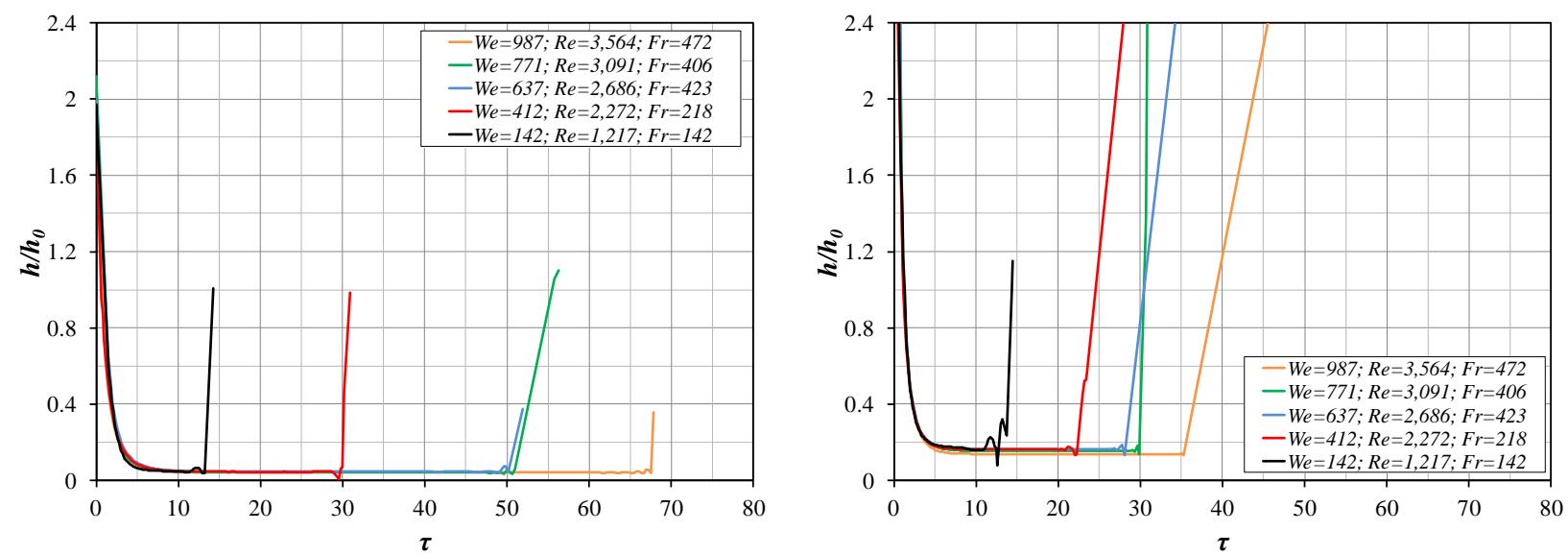

a)

b)

Figure E.18: Sub-cavity liquid film thickness ratio $h / h_{0}$ histories for varying the initial droplet condition (We, Re, and $F r)$ at a constant $h_{0}{ }^{*}$. a) $h_{0}{ }^{*}=1.0$ for Case $1-3$ and $h_{0}{ }^{*}=0.9$ for Cases 4 - 5. b) $h_{0}{ }^{*}=0.2$.

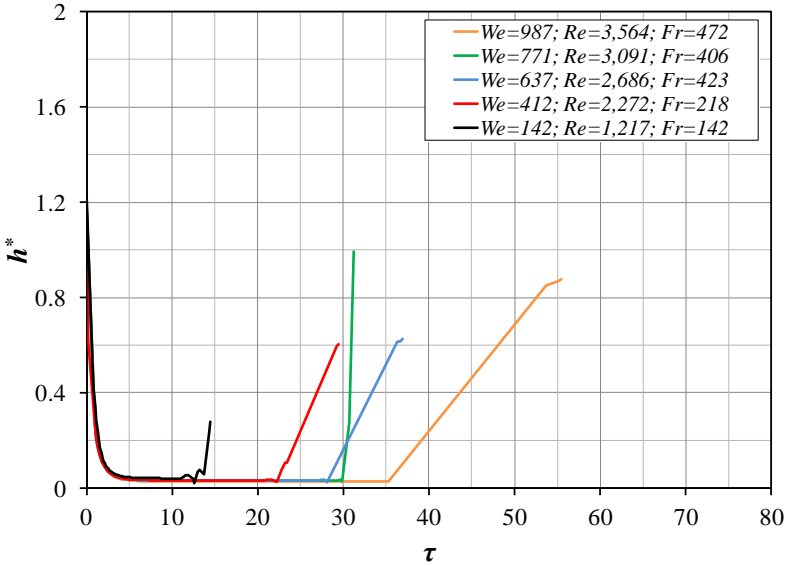

b) 

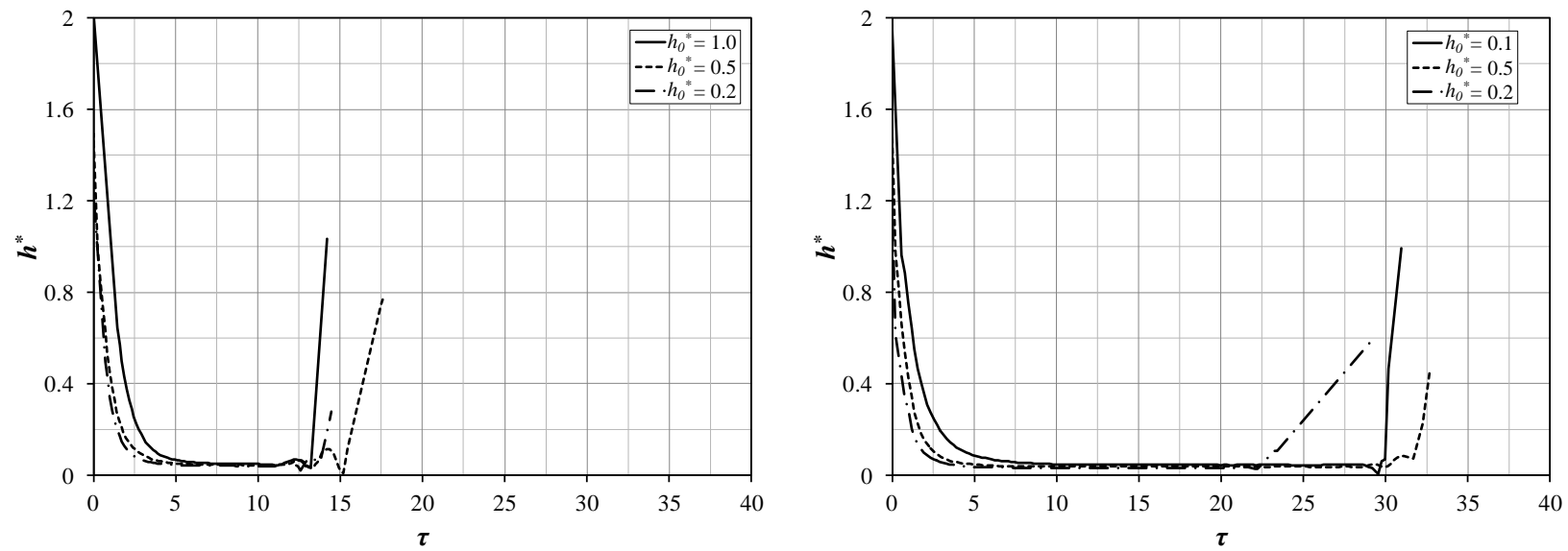

a)

b)
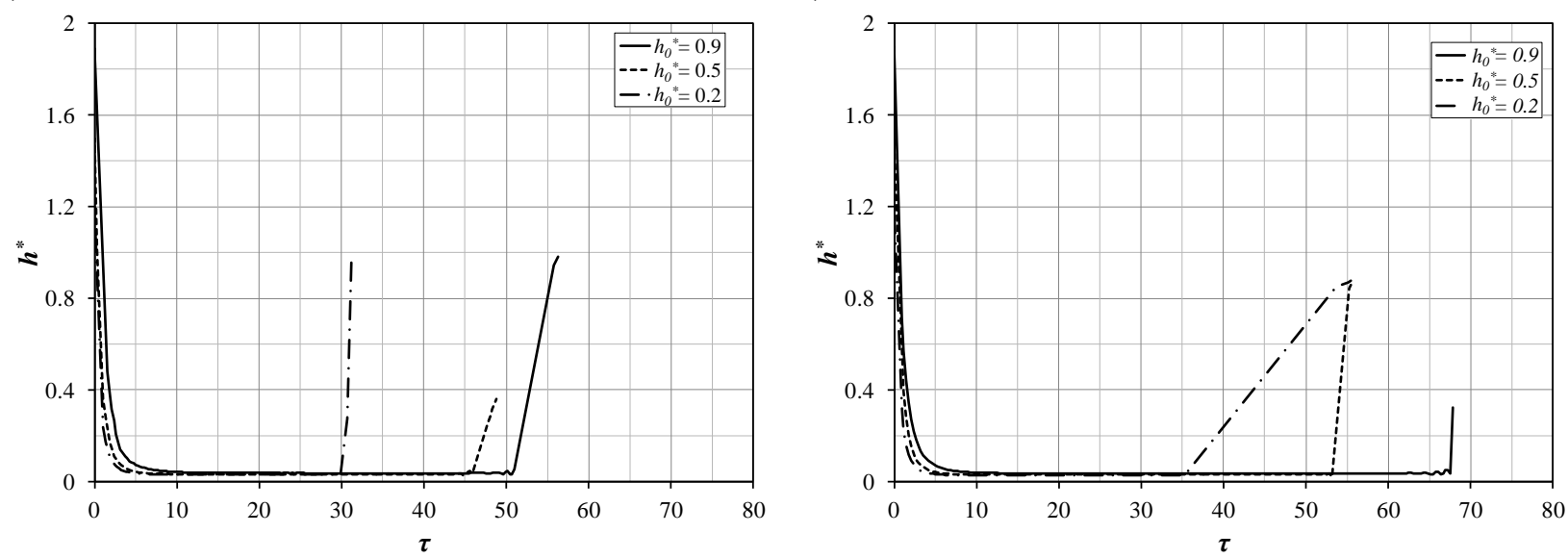

d)

Figure E.19: Dimensionless sub-cavity liquid film thickness $\left(h^{*}\right)$ histories for varying the $h_{0}{ }^{*}$ at a constant initial droplet condition (We, Re, and Fr). a) Case 1. b) Case 2. c) Case 3. d) Case 4. 

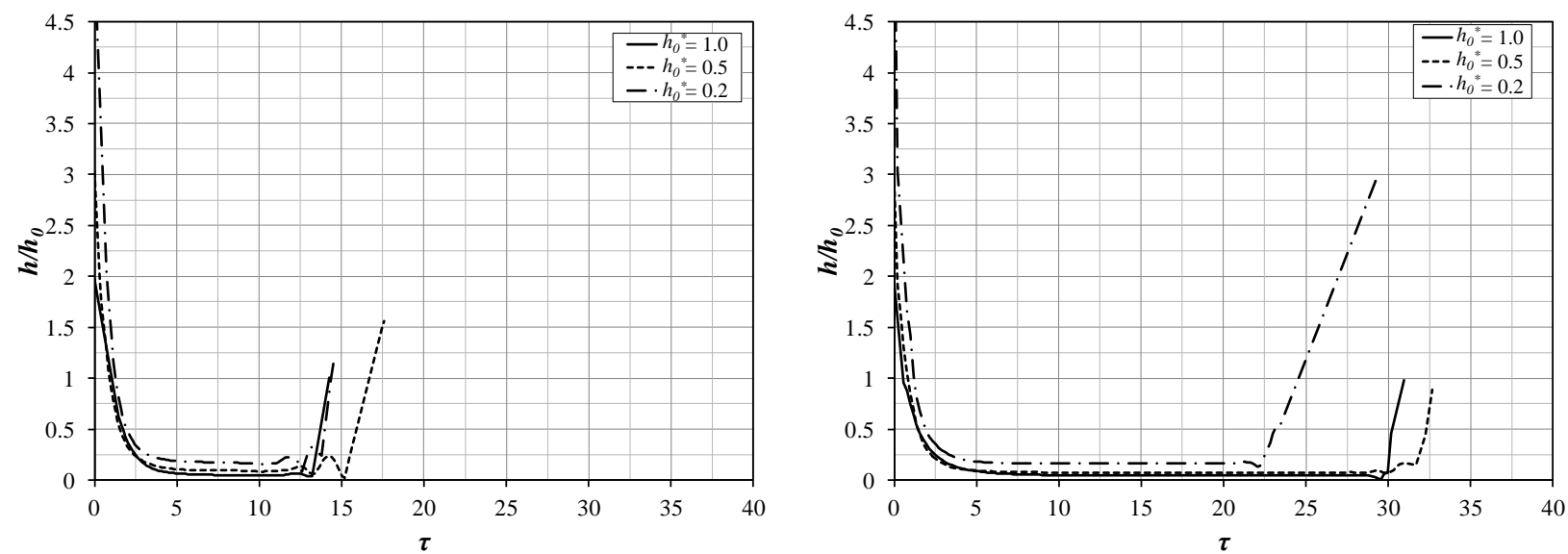

a)

b)
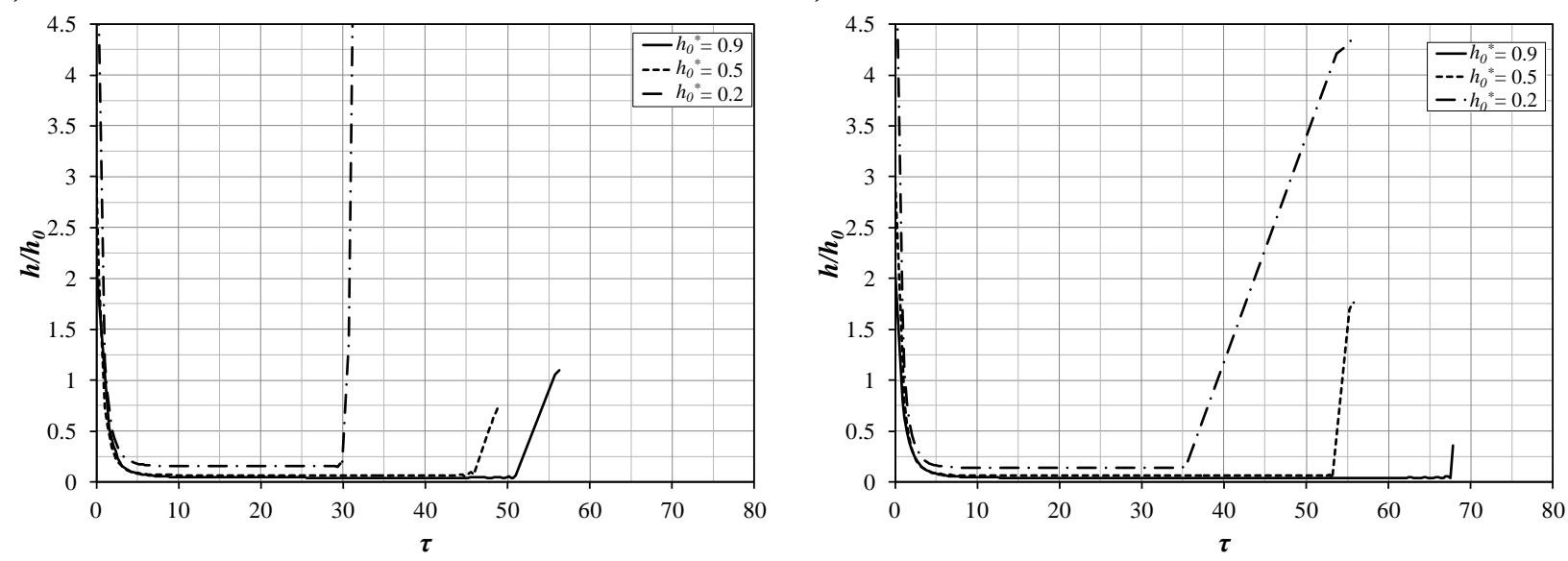

d)

Figure E.20: Sub-cavity liquid film thickness ratio $h / h_{0}$ histories for varying the $h_{0}{ }^{*}$ at a constant initial droplet condition ( $W e, R e$, and $F r)$. a) Case 1. b) Case 2. c) Case 3. d) Case 4. 